\title{
Avaliação do fator CIITA como potencial adjuvante molecular para vacinas e imunoterapias
}

Tese apresentada à Faculdade de Medicina da Universidade de São Paulo para obtenção do título de Doutor em Ciências

Programa de Patologia Orientador: Prof. Dr. Alberto José da Silva Duarte

Coorientadora: Dr. ${ }^{\text {a }}$ Telma Miyuki Oshiro 


\section{Avaliação do fator CIITA como potencial adjuvante molecular para vacinas e imunoterapias}

Tese apresentada à Faculdade de Medicina da Universidade de São Paulo para obtenção do título de Doutor em Ciências

Programa de Patologia Orientador: Prof. Dr. Alberto José da Silva Duarte

Coorientadora: Dr. ${ }^{\text {a }}$ Telma Miyuki Oshiro 
Dados Internacionais de Catalogação na Publicação (CIP)

Preparada pela Biblioteca da

Faculdade de Medicina da Universidade de São Paulo

Creprodução autorizada pelo autor

Palma, Mariana de Lucena

Avaliação do fator CIITA como potencial adjuvante molecular para vacinas e imunoterapias / Mariana de Lucena Palma. -- São Paulo, 2015.

Tese(doutorado)--Faculdade de Medicina da Universidade de São Paulo.

Programa de Patologia.

Orientador: Alberto José da Silva Duarte.

Coorientadora: Telma Miyuki Oshiro Sumida.

Descritores: 1.Proteína humana CIITA 2.Proteína transactivadora MHC de classe II 3.Proteassoma 4.Proteína humana PML 5.Proteínas modificadoras pequenas relacionadas à ubiquitina

USP/FM/DBD-422/15 


\section{No Protocolo: $0768 / 11$}

Título: "Resposta Imune Celular In Vitro Em Indivíduos Infectados Pelo Hiv-1 Induzida Por Células Dendríticas Que Expressam O Fator Ciita E A Proteína Gag De Hiv-1 Fusionado À Dc-Lamp"

Pesquisador Responsável: Dr. Alberto José da Silva Duarte Pesquisador Executante: Mariana de Lucena Palma

Co-autores: Telma Miyuki Oshiro

Finalidade Acadêmica: Doutorado

Departamento: Patologia

A Comissão de Ética para Análise de Projetos de Pesquisa - CAPPesq da Diretoria Clínica do Hospital das Clínicas da Faculdade de Medicina da Universidade de São Paulo, APROVOU / TOMOU CIÊNCIA na sessão datada de 09/11/2011, o protocolo acima.

Comentários do relator (a): "Rever na página 2 do resumo do protocolo de pesquisa na $3^{a}$ linha de baixo para cima: IFN-\&\#947; Se este é o termo mesmo, se não enviar correção".

A CAPPesq em obediência à Resolução CNS 196/96, solicita ao pesquisador (a) s elaboração de relatório parcial e final.

No caso de relatório parcial é necessário informar o tempo previsto para a conclusão do protocolo e breve resumo dos resultados obtidos.

PROF. DR. EUCLIDES AYRES DE CASTILHO

Coordenador Comissão de Ética para Análise de Projetos de Pesquisa - CAPPesq 


\section{Hospital das Clínicas da FMUSP}

Comissão de Ética para Análise de Projetos de Pesquisa

CAPPesq

$N^{\circ}$ Protocolo: 0768/11

Tí́tulo: RESPOSTA IMUNE CELULAR IN VITRO EM INDIVÍDUOS INFECTADOS PELO HIV-I INDUZIDA POR CÉLULAS DENDRÍTICAS QUE EXPRESSAM O FATOR CIITA E A PROTEÍNA GAG DE HIV-1 FUSIONADO À DC-LAMP

Pesquisador Responsável: Dr. Alberto José da Silva Duarte

Pesquisador Executante: Mariana de Lucena Palma

Finalidade Acadêmica: Doutorado

Departamento: PATOLOGIA

A Comissão de Ética para Análise de Projetos de Pesquisa - CAPPesq da Diretoria Clínica do Hospital das Clínicas da Faculdade de Medicina da Universidade de São Paulo, APROVOU / TOMOU CIÊNCIA na sessão datada de 25/04/2012, do(s) documento(s) abaixo mencionado(s):

- Carta datada de 29.02.12:

- Alteração no processo de obtenção de amostras de sangue periférico de pacientes a serem utilizadas no projeto e novo Termo de Consentimento Livre e Esclarecido.

A CAPPesq em obediência à Resolução CNS 196/96, solicita ao pesquisador (a) s elaboração de relatório parcial e final.

No caso de relatório parcial é necessário informar o tempo previsto para a conclusão do protocolo e breve resumo dos resultados obtidos.

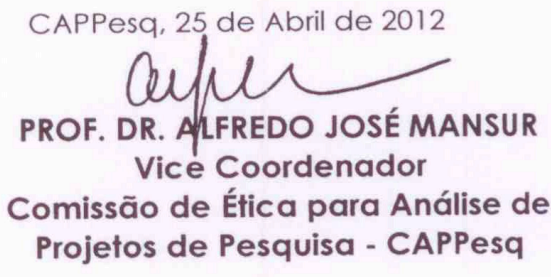

Rua Dr. Ovídio Pires de Campos, 225 - Prédio da Administração - $5^{\circ}$ andar - CEP 05403-010 - São Paulo - SP Fone: $55112661-6442$ ramais 16, 17, 18 e 20 - e-mail: cappesq@hcnet.usp.br 
To my beloved parents,

Israel and Cilda Palma. 


\section{AKNOWLEDGEMENTS}

My most cordial thanks to the Laboratory of Dermatology and Immunodeficiency LIM-56, the University of São Paulo Medical School, the Center for Vaccine Research and the University of Pittsburgh, for the technical, financial and human support.

I also would like to thank FAPESP for the granted scholarships.

Immeasurable appreciation and deepest gratitude for the help and support are extended to the following persons who in one way or another have contributed in making this study possible.

Dr. Alberto Duarte, my research advisor, for providing me with the invaluable opportunity to pursue my academic goals and for the precious theoretical guidance.

Dr. Ernesto Marques, my mentor at the Center for Vaccine Research, for accepting me as a visiting researcher in his laboratory where I grew professionally and performed important steps of my research. His timely advice, meticulous scrutiny and scientific approach have helped me to a very great extent to accomplish this task.

Dra. Telma Oshiro, my co-adviser, for her keen interest in me at every stage of my research and her promptness to help me at any time. Her inspirations, timely suggestions with kindness, enthusiasm, modesty and respect have enabled me to complete my thesis.

Dr. Bruno Douradinha, for the constant theoretical and technical support during the critical phases of my research, for his fundamental collaboration in this research and mainly for his positivity and friendship that helped keep me strong until the end of my work.

Dr. Eduardo Nascimento, for his kind help and co-operation throughout my study period.

Dr. Robbie Mailliard and Dr. Rafael Dhalia, for providing me necessary technical suggestions during my research pursuit.

In special, to Dra. Isabelle Viana, dear friend that during my period abroad was my mom, my sister, my advisor, my co-worker and my exploration company. We were two 
peas in a pod. I am immensely grateful for being able to learn with you about work, life and love! You are the best!

Dra. Paula Rigato and Dra. Liã Arruda, for the technical suggestions, friendship and kind advises during difficult times.

To my friends from the Laboratory of Dermatology and Immunodeficiency - LIM-56, Denise Reis, Bruna Santillo, Wanessa Cardoso and Laís Teodoro, for the cooperation, laughs and good times. In special, to Marcella Vassão and Ana Paula Vieira, my "paulistana" family that has a special place in my heart. I cannot thank them enough for all the help during these four years.

To my friends from the Center for Vaccine Research, Brandon Deshmukh, Andrea Dobbs, Lucianna Freitas, Guangchao Gu, Priscila Castanha, Yang Gao, Cintia Marinho and Jeff Meier for the kind help, cultural exchanges and good times.

To the staffs of LIM-56, the University of São Paulo Medical School and the Center for Vaccine Research, for all the support they have given me throughout the periods I spent at each institution.

I also would like to express all my love and infinite thanks to the following persons to whom I am greatly indebted.

Israel and Cilda Palma, my adored parents, who have been a source of never ending encouragement and inspiration to me throughout my life. I cannot begin to express my gratitude for the myriad of ways in which, throughout my life, you both have actively supported me in my determination to find and realize my potential, always nurturing me with the purest love I could ever wish for. 
Marina Palma and João Paulo Matos, my sister and brother-in-law, for bringing me joy and lightheartedness. Their wedding was a high point during these four years.

To all my relatives, for their unwavering love and emotional support.

To the sisters life provided me with, Mylla Pimentel, Juliana Santos, Amaranta Muniz and Patrícia Toniolo, who endlessly prove that they want be part of my life regardless of the vast distance between us. I am so glad you never gave up on us!

Paul and Angela Lamers, for being the best "gringos" I have ever met. Their welcoming attitude toward me into their lives is so sweet and sincere that makes me realize how benevolent life is to me. And I cannot forget to thank them for all the pizza nights!

Stefan Lamers, my boyfriend and the best surprise this doctoral endeavor gave me, who brought bright colors, calming fragrances and never-ending caring to my student life, helping me face all the challenges and down sides with positivity and the confidence that I would always count on him for. You definitely provided me with the best time of my life! As if all that were not enough, you also patiently helped me with the grammatical corrections, so thanking you is not even close to enough!

Once again, my thanks goes out to everyone who helped me along the way! 
"You must plant your own garden and decorate your own soul, instead of waiting for someone to bring you flowers. And you learn that you really can endure. You really are strong. And you can go so farther than you thought you could go. And that life really has a value."

(William Shakespeare) 


\section{TABLE OF CONTENTS}

List of abbreviations and acronyms

List of figures

List of tables

Resumo

Abstract

1 INTRODUCTION AND JUSTIFICATION

2 OBJECTIVES

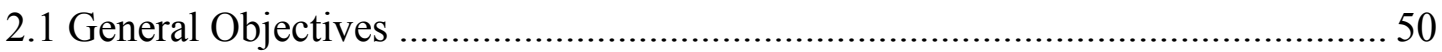

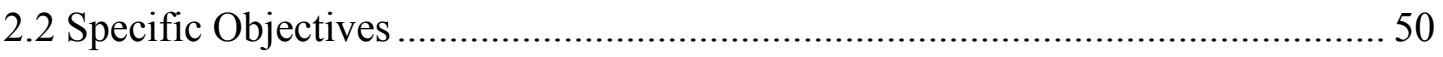

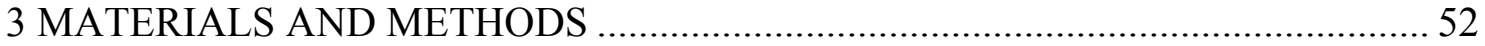

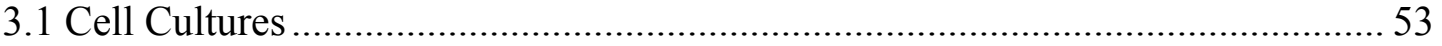

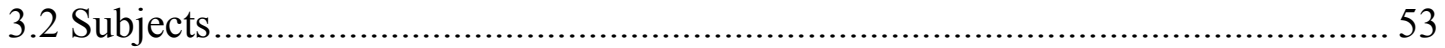

3.3 Monocytes isolation and cryopreservation ......................................................... 54

3.4 Generation of monocyte-derived dendritic cells ................................................ 55

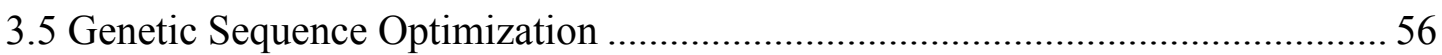

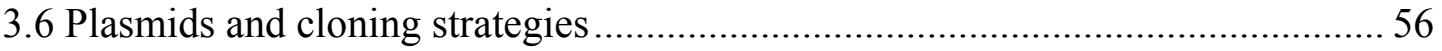

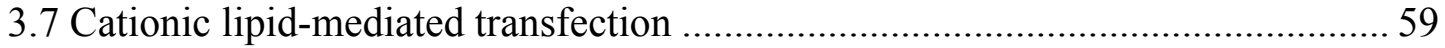

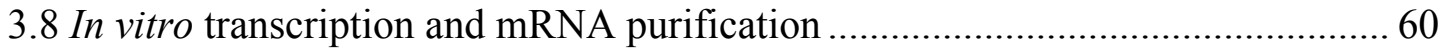

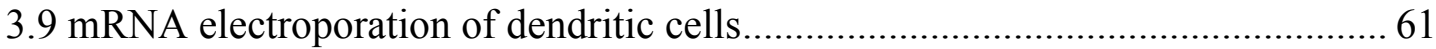

3.10 Generation of Lentiviral Gene Transduction Vector Particles .......................... 61

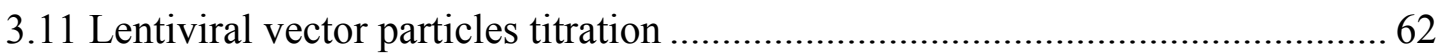

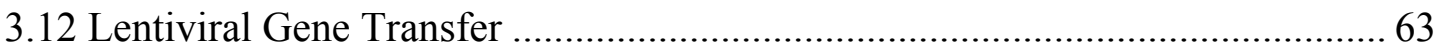

3.13 Inoculation of healthy human skin explant with lentiviral vectors .................... 64

3.14 Immunofluorescence and Confocal Microscopy …............................................ 65

3.15 Cell lysates preparation and total protein quantification ................................... 66

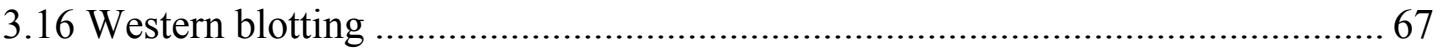

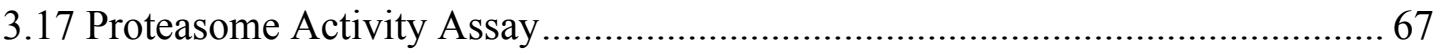

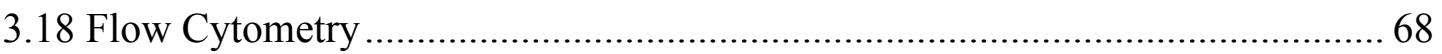


3.18.1 Surface staining of human cell lines

3.18.2 Surface and intracellular detection of MHC II molecules in human cell lines

3.18.3 Gating strategy for human cell lines analysis...................................... 70

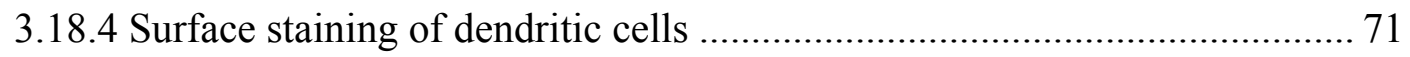

3.18.5 Surface and intracellular detection of MHC II molecules in dendritic cells 72

3.18.6 Gating strategy for dendritic cells analysis............................................ 72

3.19 Dendritic cells activation and Interleukin-12(p70) production ......................... 73

3.20 RNA Isolation and Quantitative Real-Time PCR ............................................. 74

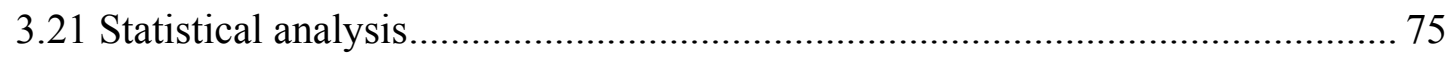

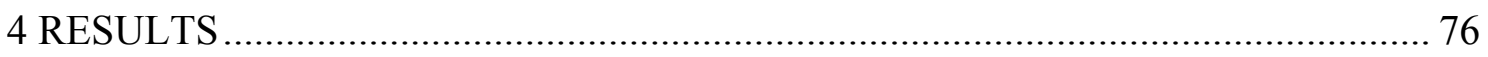

4.1 CIITA sequence design and optimization for expression in human cells ............ 77

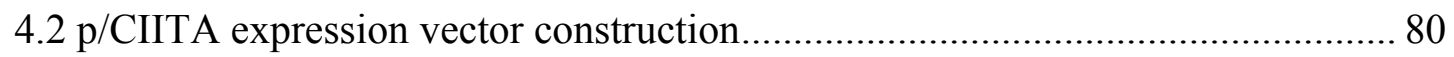

4.3 CIITA protein expression and intracellular distribution in p/CIITA-transfected

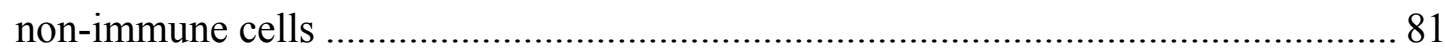

4.4 CIITA transactivation activity in transfected non-immune cells.......................... 85

4.5 Kinetics of CIITA protein expression in transfected non-immune cells ............... 88

4.6 Influence of proteasomal degradation in the levels of CIITA expression by non-

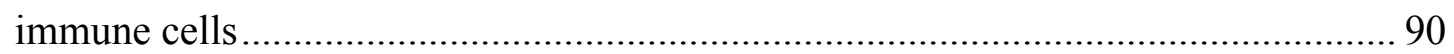

4.7 Evaluation of PML proteins expression by non-immune cells............................ 92

4.8 CIITA protein expression in p/CIITA-transfected mice cells ............................. 95

4.9 CIITA gene delivery to human dendritic cells ...................................................... 96

4.9.1 Establishment and characterization of DC cultures..................................... 97

4.9.2 Expression of endogenous CIITA protein by DCs ................................... 102

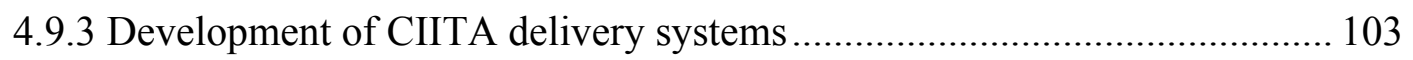

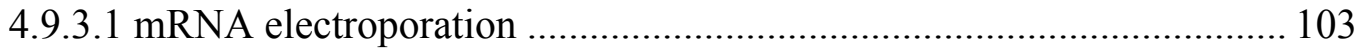

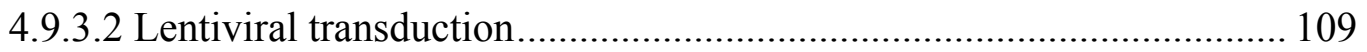

4.9.3.3 Lentiviral transduction of human cell lines ....................................... 115

4.9.3.4 Lentiviral transduction of DCs ....................................................... 118

4.9.3.5 Lentiviral inoculation to human skin explant .................................. 124

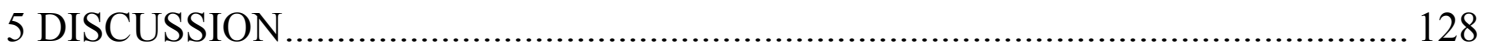




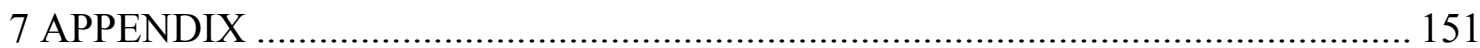

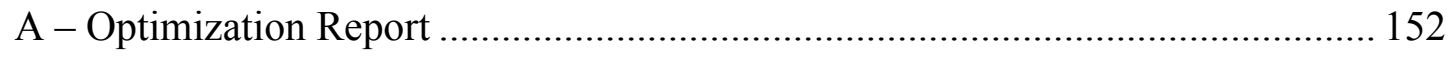

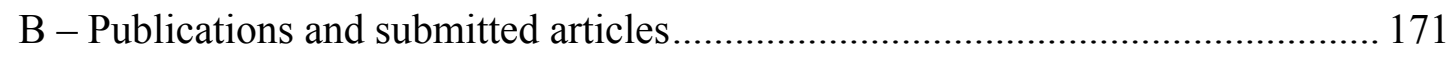

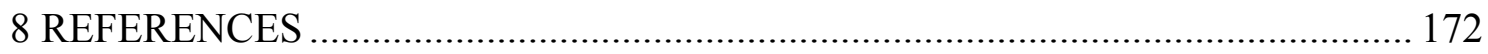




\section{LIST OF ABBREVIATIONS AND ACRONYMS}

AAV Adeno-Associated Virus

AD Activation Domain

AIDS Acquired Immune Deficiency Syndrome

ANOVA Analysis of Variance

APC Antigen Presenting Cell

AT Acetyltransferase

AT-2 Aldrithiol-2

ATPase Adenosine Triphosphatase

BCA Bicinchoninic Acid

BLIMP-1 B-Lymphocyte-Induced Maturation Protein 1

BLS Bare Lymphocyte Syndrome

BRG1 Brahma-Related Gene 1

BS Blocking Solution

BSA Bovine Serum Albumin

cAMP Cyclic Adenosine Monophosphate

CARD Caspase-recruitment Domains

CARM1 Coactivator-associated Arginine Methyltransferase 1

cART Combination Antiretroviral Therapy

CBP CREB-binding Protein

CCR7 C-C Chemokine Receptor 7

CD Cluster of Differentiation

CD40L CD40 Ligand

cDNA Complementary Deoxyribonucleic Acid

ChIP-seq Chromatin Immunoprecipitation Sequencing

CIITA Class II Transactivator

CMV Cytomegalovirus

CpG Cytidine-Phosphate-Guanosine

CREB cAMP Responsive Element Binding Protein

CTL Cytotoxic T Lymphocyte

DAPI 4',6-diamidino-2-phenylindole 


\begin{tabular}{|c|c|}
\hline $\mathrm{DC}$ & Dendritic Cell \\
\hline DMEM & Dulbecco`s Modified Eagle`s Medium \\
\hline DMSO & Dimethyl Sulfoxide \\
\hline DNA & Deoxyribonucleic Acid \\
\hline DPBS & Dulbecco`s Phosphate-Buffered Saline \\
\hline dsRNA & Double stranded Ribonucleic Acid \\
\hline EDTA & Ethylenediamine Tetraacetic Acid \\
\hline eGFP & Enhanced Green Fluorescent Protein \\
\hline EIA/RIA & Enzyme Immunoassay/ Radioimmunoassay \\
\hline ELISA & Enzyme-Linked Immunosorbent Assay \\
\hline ERK & Extracellular Signal Regulated Kinase \\
\hline FasL & Fas Ligand \\
\hline FBS & Fetal Bovine Serum \\
\hline FITC & Fluorescein Isothioyanate \\
\hline FMDV & Foot-and-Mouth Disease Virus \\
\hline FSC & Forward Scatter \\
\hline GDB & GTP Binding Domain \\
\hline GM-CSF & Granulocyte Macrophage Colony-stimulating Factor \\
\hline gp120 & Glycoprotein 120 \\
\hline HDAC & Histone Deacetylase \\
\hline HEK293 & Human Embryonic Kidney 293 cells \\
\hline HEPES & 4-(2-hydroxyethyl)-1-piperazineethanesulfonic Acid \\
\hline HIV & Human Immunodeficiency Virus \\
\hline HLA & Human Leukocyte Antigen \\
\hline iDC & Immature Dendritic Cell \\
\hline IFN & Interferon \\
\hline $\operatorname{Ig}$ & Immunoglobulin \\
\hline $\mathrm{Ii}$ & Invariant chain \\
\hline $\mathrm{IkB}$ & Inhibitor of kappa B \\
\hline IMDM & Iscove`s Dubecco`s Modified Medium \\
\hline IRES & Internal Ribosome Entry Site \\
\hline JAK & Janus Kinase \\
\hline LNGFR & Low Affinity Nerve Growth Factor Receptor \\
\hline
\end{tabular}




\begin{tabular}{|c|c|}
\hline LRR & Leucine Rich Region \\
\hline LTR & Long Terminal Repeat \\
\hline LV & Lentivirus \\
\hline $\mathrm{mAb}$ & Monoclonal Antibody \\
\hline MAPK & Mitogen-activated Protein Kinase \\
\hline MCS & Multiple Cloning Site \\
\hline MCLR & Mannose C-type Lectin Receptor \\
\hline $\mathrm{mDC}$ & Mature Dendritic Cell \\
\hline MEM & Minimum Essential Media \\
\hline MFI & Median Fluorescence Intensity \\
\hline $\mathrm{MHC}$ & Major Histocompatibility Complex \\
\hline MHC2TA & Major Histocompatibility Complex class 2 Transactivator \\
\hline miRNA & Micro-Ribonucleic Acid \\
\hline mo-DC & Monocyte-derived Dendritic Cell \\
\hline MOI & Multiplicity of Infection \\
\hline mRNA & Messenger Ribonucleic Acid \\
\hline $\mathrm{NF}-\kappa \mathrm{B}$ & Nuclear Factor- $\kappa \mathrm{B}$ \\
\hline NFY & Nuclear Factor Y \\
\hline NLR & Nod-like Receptor \\
\hline NLRC5 & NLR Family, CARD Domain Containing 5 \\
\hline OPT & Optimized \\
\hline ORF & Open Reading Frame \\
\hline p-PKR & Phospho-protein Kinase R \\
\hline $\mathrm{P}-\mathrm{TEFb}$ & Positive Transcription Elongating Factor b \\
\hline PBMC & Peripheral Blood Mononuclear Cells \\
\hline PBS & Phosphate-buffered Saline \\
\hline PCAF & p300/CBP-associated Factor \\
\hline PCR & Polymerase Chain Reaction \\
\hline $\mathrm{PE}$ & Phycoerythrin \\
\hline $\mathrm{PGE}_{2}$ & Prostaglandin $\mathrm{E}_{2}$ \\
\hline PKA & Protein Kinase A \\
\hline PKR & Protein Kinase R \\
\hline PML & Promyelocytic Leukemia \\
\hline
\end{tabular}


PMSF Phenylmethanesulfonyl Fluoride

Poly-I:C Polyinosinic:polycytidylic Acid

PRE Post-transcriptional Regulatory Element

PST Proline/Serine/Threonine domain

PTM Post-translational Modification

PVDF Polyvinylidene Fluoride

qRT-PCR Quantitative Real Time Polymerase Chain Reaction

RFX Regulatory Factor X complex

Rhu Recombinant Human

RIPA Radioimmunoprecipitation Assay

RNA Ribonucleic Acid

RPMI Roswell Park Memorial Institute medium

RRE Rev Eesponse Element

RSI Relative Signal Intensity

RT Room Temperature

SD Standard Deviation

SDS Sodium Dodecyl Sulfate

SDS-PAGE Sodium Dodecyl Sulfate -Polyacrylamide Gel Electrophoresis

SEM Standard Error of the Mean

SFFV Spleen Focus-Forming Virus

siRNA Small Interfering Ribonucleic Acid

SOCS Suppressor of Cytokine Signaling

SSC Side Scatter

ssRNA Single Strand Ribonucleic Acid

STAT Signal Transducer and Activator of Transcription

SUMO Small Ubiquitin-like Modifier

TAF Transcription Activator Factor

TBP TATA-binding Protein

TF Transcription Factor

Th T helper

TLR Toll-like Receptor

TMB Tetramethylbenzidine

TNF- $\alpha \quad$ Tumor Necrosis Factors alpha 
Treg

T regulatory cells

tRNA Transfer Ribonucleic Acid

UTR Untranslated Region

VSV-G Glycoprotein G of the Vesicular Stomatitis Virus 


\section{LIST OF FIGURES}

Figure 1 - MHC II genes transactivation by CIITA _..................................................... 29

Figure 2 - Regulatory regions of the gene encoding CIITA ..................................... 31

Figure 3 - Gating strategies for the analysis of human cell lines expressing CIITA ..... 71

Figure 4 - Gating strategies for dendritic cells phenotypic analysis ........................... 73

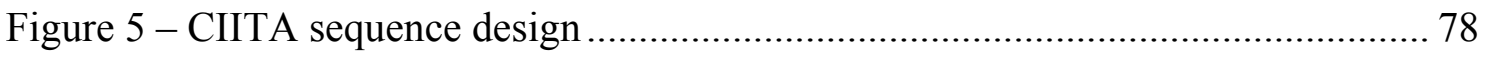

Figure 6-Optimization of CIITA_2A sequence for improved expression in human cells

Figure 7 - Strategy for constructing the CMV expression vector $\mathrm{p} / \mathrm{CIITA}$................... 81

Figure 8 - Transfected HEK293, HeLa and Huh-7 cell lines efficiently express the

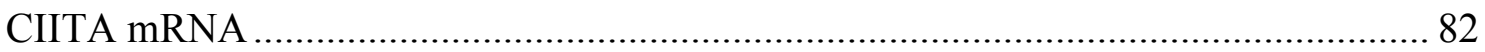

Figure 9 - CIITA is expressed in transfected HEK293, HeLa and Huh-7 cell lines with proper intracellular distribution

Figure $10-$ p/CIITA-transfected cell lines express MHC II molecules at distinct levels and kinetics 87

Figure $11-$ p/CIITA-transfected HEK293 cells do not express costimulatory molecules 88

Figure $12-\mathrm{p}$ /CIITA-transfected cells differ in the level and kinetic of CIITA expression

Figure 13 - p/CIITA-transfected cells present distinct CIITA degradation kinetics due to heterogeneous levels of proteasome degradation

Figure $14-\mathrm{p}$ /CIITA-transfected cells differentially regulate the PML monoSUMOylation.

Figure $15-$ p/CIITA-transfected mice cells are able to express the transcriptionally active human CIITA protein 96

Figure 16 - Phenotypic characterization of monocyte-derived dendritic cells 99 
Figure 17 - Mature dendritic cell subtypes are phenotypically similar 100

Figure 18 - Activated dendritic cell subtypes significantly differ in the secretion of IL12(p70).

Figure 19 - Expression of endogenous CIITA protein by iDCs and mDC subtypes... 102

Figure 20 - pMACS constructs used for in vitro mRNA synthesis 104

Figure 21 - In vitro transcription of eGFP and LNGFR mRNAs 105

Figure 22 - Attempts to obtain a linear template for CIITA mRNA transcription

Figure 23 - eGFP expression and viability of immature dendritic cells electroporated with different electric forces 108

Figure 24 - Schematical representation of the lentiviral vector system for CIITA sequence delivery

Figure $25-\mathrm{SiEW}-\mathrm{CIITA}$ vector construction

Figure 26 - Transfer vectors construction using the LeGO-iG2 plasmid 114

Figure 27 - LV-CIITA-transduced cell lines express MHC II molecules at distinct levels and kinetics.

Figure 28 - The lentiviral vector LV-CIITA efficiently transduces monocytes from healthy individuals without significant CIITA-specific induction of MHC II molecules in iDCs 120

Figure 29 - The lentiviral vector LV-CIITA efficiently transduces monocytes from HIV positive individuals without significant CIITA-specific induction of MHC II molecules in iDCs

Figure 30 - Monocytes and iDCs express lower levels of mono-SUMOylated PML proteins than HEK293 cells

Figure 31 - The lentiviral vector LV-CIITA efficiently transduces primary human cells in skin explants and promotes CIITA expression. 126

Figure 32 - A proposed mechanistic model for the post-translational regulation of CIITA in non-immune cells. 


\section{LIST OF TABLES}

Table 1 - Sequences of primers used in PCR reactions 


\section{RESUMO}

Palma ML. Avaliação do fator CIITA como potencial adjuvante molecular para vacinas e imunoterapias [tese]. São Paulo: Faculdade de Medicina, Universidade de São Paulo; 2015.

O fator CIITA é a proteína responsável por controlar a transcrição de genes do complexo principal de histocompatibilidade de classe II (MHC II) envolvidos na apresentação antigênica a linfócitos $\mathrm{T} \mathrm{CD}^{+}$. A expressão desta proteína é complexa e célula-específica, dependendo de mecanismos de regulação transcricionais e póstranscricionais. Com o intuito de investigar o potencial do fator CIITA como adjuvante molecular, no presente estudo desenvolvemos e validamos sistemas de transferência gênica capazes de promover a eficiente expressão de CIITA em vários tipos celulares. Além disso, investigamos a regulação pós-traducional deste fator em células não hematopoéticas. Desta forma, foram produzidos um vetor plasmidial e um vetor lentiviral, ambos carreando a sequência do fator CIITA humano desenhada in silico visando a eliminação de elementos cis-reguladores, e otimizada para eficiente expressão em células humanas. A transfecção/transdução de três linhagens de células humanas não hematopoéticas resultou na eficiente expressão de CIITA com localização nuclear apropriada. Células expressando CIITA apresentaram síntese de novo do MHC II, confirmando a funcionalidade da proteína e validando ambos os vetores para a análise futura da atividade adjuvante do CIITA em imunizações gênicas. Ensaios preliminares de inoculação de explantes de pele humana com o vetor lentiviral evidenciaram a eficiente transdução e expressão do CIITA exógeno em células primárias. Em seguida, células dendríticas (DCs) derivadas de monócitos de indivíduos saudáveis ou infectados com HIV-1 foram transduzidas com o vetor lentiviral para confirmar a expressão do CIITA em células primárias e avaliar a aplicação desse sistema adjuvante no aprimoramento da vacina de DCs anti-HIV. DCs de indivíduos saudáveis ou infectados foram transduzidas com sucesso pelo lentivírus, o qual induziu uma produção prolongada do mRNA codificando CIITA. Entretanto, os vetores lentivirais induziram um aumento inespecífico da expressão de marcadores fenotípicos das DCs, incluindo as moléculas do MHC II, o que impediu a avaliação indireta da expressão e atividade do fator CIITA através da detecção da expressão aumentada do MHC II. Ensaios futuros 
irão avaliar se o fator transcricional é expresso pelas DCs transduzidas ou se essas células apresentam um controle mais restrito da expressão do CIITA comparadas às linhagens celulares avaliadas. Interessantemente, ensaios de western blot comparativos entre as três linhagens de células humanas transfectadas/transduzidas, juntamente com ensaios de inibição da degradação protéica pelo inibidor do proteassoma, nos permitiu descrever um novo mecanismo de regulação pós-traducional do CIITA. Aqui, nós identificamos que cada tipo de célula não hematopoética mantém níveis específicos da proteína, e portanto, da sua atividade transcricional, através da regulação da degradação do CIITA pelo proteassoma. Essa regulação é mediada pela modulação dos níveis das proteínas da leucemia promielocítica (PML) acopladas a proteínas SUMO (modificadores pequenos similares à ubiquitina), modificação pós-traducional requerida para a interação PML-CIITA que impede a degradação pelo proteassoma. Esse novo mecanismo aqui descrito contribui para o entendimento ainda incipiente da regulação pós-traducional do fator CIITA em células não hematopoéticas e pode ter implicações importantes na aplicação dessa proteína como adjuvante molecular para imunoterapias.

Descritores: proteína humana CIITA; proteína transativadora MHC de classe II; proteassoma; proteína humana PML; proteínas modificadoras pequenas relacionadas à ubiquitina. 


\begin{abstract}
Palma ML. Evaluation of CIITA factor as a potential molecular adjuvant for vaccines and immunotherapies [thesis]. São Paulo: "Faculdade de Medicina, Universidade de
\end{abstract} São Paulo"; 2015

The CIITA factor is a protein responsible for controlling the transcription of major histocompatibility complex class II (MHC II) genes involved on antigen presentation to $\mathrm{CD}^{+} \mathrm{T}$ helper cells. The expression of this transcription factor is complex and differs in various cell types depending on transcriptional and post-transcriptional regulatory mechanisms. In order to investigate the CIITA factor potential as molecular adjuvant, here we developed and validated two gene delivery systems capable of promoting efficient CIITA expression in various human cell types. Additionally, we applied the delivery systems to investigate the post-translational regulation of this factor in nonimmune cells. A DNA plasmid and a lentiviral vector were produced, both carrying the human CIITA DNA sequence in silico designed to avoid cis-regulatory elements, and genetic optimized for expression efficacy in human cells. Transfection or transduction of three different non-immune human cell lines resulted in efficient CIITA expression with proper nuclear localization. The CIITA-expressing cells presented de novo MHC II molecules expression confirming the functionality of the exogenous protein, and validating both delivery systems for the future analysis of the CIITA adjuvant activity in genetic immunizations. Preliminary assays involving the inoculation of the lentiviral vector into human skin explants showed efficient transduction and expression of exogenous CIITA in primary cells. Next, monocyte-derived dendritic cells (DCs) from healthy individuals and HIV-1-infected patients were transduced with the lentiviral vector to confirm the exogenous CIITA expression in primary human cells and also evaluate the applicability of this adjuvant system to improve the DC-based vaccines against HIV. DCs from healthy and infected individuals were successfully transduced by the lentivirus, which induced a sustained CIITA mRNA production. However, the vector particles by themselves induced an unspecific upregulation of DC's phenotypic surface markers, including the MHC II molecules, impairing our strategy to indirectly evaluate CIITA expression and activity through the detection of MHC II enhanced expression. Further investigations are necessary to confirm whether the transcription factor is efficiently expressed in transduced DCs or if these cells present a more restrict 
control of CIITA protein expression than the evaluated non-immune cells. Interestingly, western blot assays comparing the three human cell lines, transfected or transduced, along with inhibition of protein degradation by proteasome inhibitor treatments, allowed us to describe a new and intricate mechanism of CIITA post-translational regulation. Here we identified that each non-immune cell type maintain specific protein levels, and hence transcriptional activity, by modulating the rate of CIITA proteasomal degradation. This modulation is achieved by controlling the levels of Promyelocytic Leukemia (PML) proteins attached to Small Ubiquitin-like Modifier (SUMO) proteins, a post-translational modification required for the PML-CIITA interaction, which impairs the proteasomal degradation. This new mechanism described here contributes to the developing understanding of the CIITA post-translational regulation in non-immune cells, and might have important implications in the use of this transcription factor as a molecular adjuvant for immunotherapies.

Descriptors: CIITA protein, human; MHC class II transactivator protein; proteasome; PML protein, human; small ubiquitin-related modifier proteins. 
1 INTRODUCTION AND JUSTIFICATION 
Class II transactivator (CIITA) was initially identified in patients with a rare hereditary disease called bare lymphocyte syndrome (BLS) characterized by a complete loss of major histocompatibility complex class II (MHC II) expression and severe deficiency in $\mathrm{CD}^{+} \mathrm{T}$ cell-dependent adaptive immunity ${ }^{1}$. Complementation experiments with CIITA cDNA rescued the normal phenotype and established this protein as the main regulator of MHC II transcriptional activation ${ }^{2}$. Later, the expression of other genes encoding accessory proteins required for MHC II-restricted antigen presentation was also shown to be regulated by CIITA, which is therefore a central factor to controlling the response to foreign antigens . $^{3,4}$.

CIITA controls the expression of MHC II molecules displayed on the surface of antigen presenting cells (APCs) that present peptides to $\mathrm{T}$ cells to initiate an adaptive immune response. The major target genes of CIITA include classic MHC II molecules (human leukocyte antigen [HLA]-DR, HLA-DP, and HLA-DQ), but also additional non-classic genes associated with antigen processing and selection including HLA-DM, HLA-DO and cluster of differentiation (CD)74 (invariant chain) ${ }^{5-7}$. This coordinated regulation of MHC II and other genes necessary for its function is quite unique, thus CIITA has been termed the "master regulator" of MHC II and antigen presentation"

The classic and non-classic MHC II genes expression is regulated by CIITA through complex mechanisms including chromatin remodeling, transcription initiation and elongation ${ }^{3}$. However, it does not directly bind DNA. Rather, the CIITA is recruited to the MHC II promoters through protein-protein interactions with components of a stable macromolecular nucleoprotein complex referred to as the MHC II enhanceosome. This complex includes the regulatory factor $\mathrm{X}$ complex (RFX5, RFXANK and RFXAP), the cyclic adenosine monophosphate (cAMP) responsive element binding protein (CREB) and the nuclear factor $\mathrm{Y}$ (NFYA/B/C subunits). These transcription 
factors cooperatively bind DNA through the SXY module, a highly constrained series of sequences (S-X-X2-Y boxes) typically located within 300 base pairs (bp) of the MHC II genes transcriptional initiation site ${ }^{3,8,9}$. Although all enhanceosome components are expressed ubiquitously, their binding to the MHC II gene is not sufficient to support $\operatorname{expression}^{10}$. The interaction of CIITA with each of these DNA-bound transcription factors is thus required for the transcriptional activation ${ }^{11,12}$ (Figure 1). SXY modules are also found in the MHC class I region where CIITA is recruited but has a secondary role, the major transactivator here being the NLR Family, CARD Domain Containing 5 (NLRC5) factor ${ }^{13}$.

Within the enhanceosome, CIITA operates recruiting components from the transcription-initiation machinery (transcription factor IID [TFIID] and TFIIB) ${ }^{14,15}$ and interacting with the positive transcription elongating factor $\mathrm{b}(\mathrm{P}-\mathrm{TEFb})^{16}$. The transactivator also recruits the chromatin-remodeling factor brahma-related gene 1 $(\mathrm{BRG} 1)^{17}$ and various histone modifying enzymes such as acetyltransferases (ATs) p300, CREB-binding protein (CBP) and p300/CBP-associated factor (PCAF), as well as the coactivator-associated arginine methyltransferase 1 (CARM1), all of which remodel the chromatin to support active transcription ${ }^{18}$. In addition, CIITA has been reported to contain an endogenous histone-acetylase activity ${ }^{19}$. Therefore, while it does not directly bind DNA, the CIITA protein serves to nucleate and coordinate the various transcription factors and chromatin modifying enzymes that are necessary to support transcription. Consequently, the regulation of MHC II molecules expression precisely parallels the regulation of CIITA expression and activity (Figure 1). 


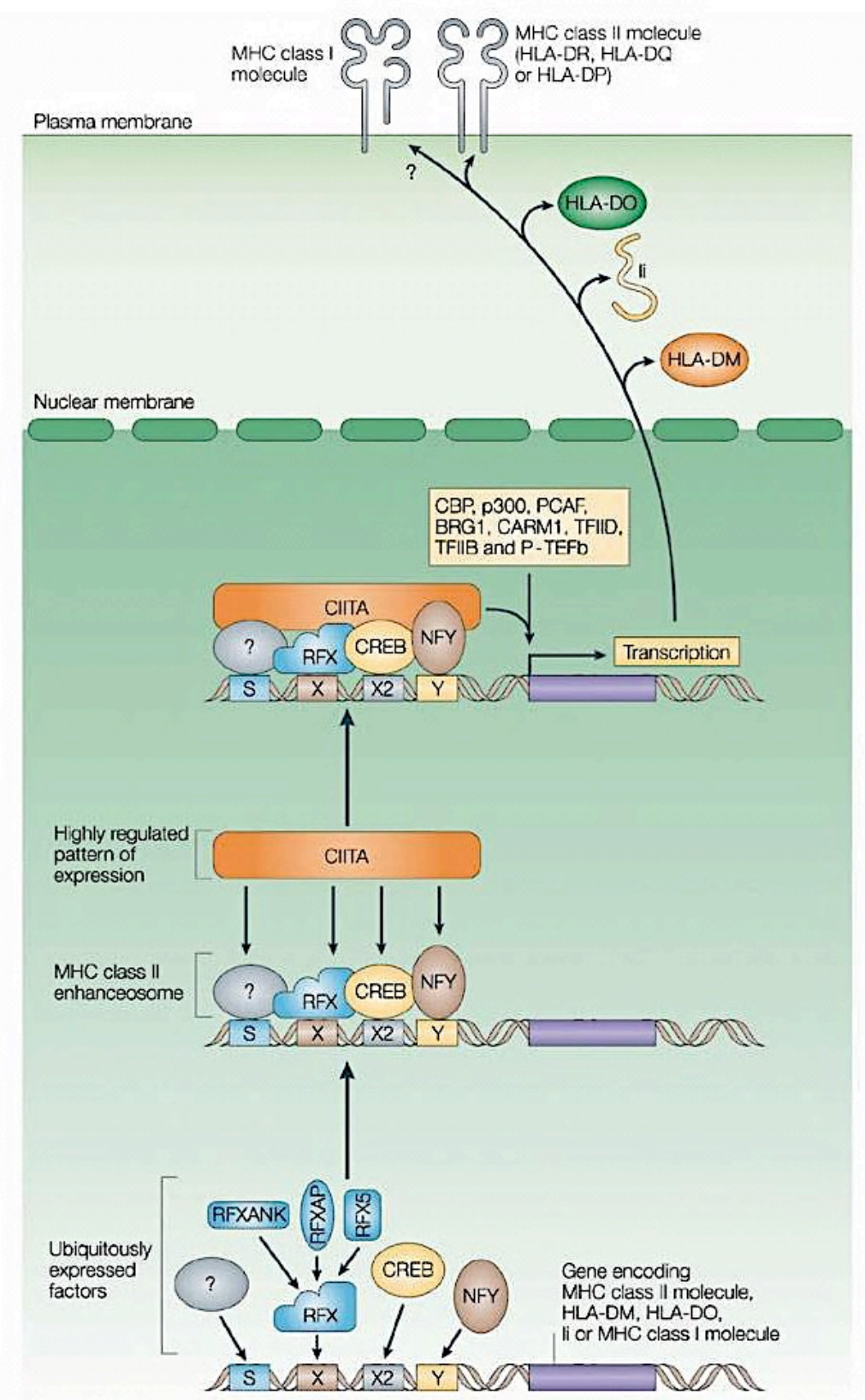

SOURCE: Reith et al., $2005^{3}$

Figure 1 - MHC II genes transactivation by CIITA. The expression of MHC class II and class I molecules, as well as the accessory protein HLA-DM, HLA-DO and invariant chain, is controlled by promoter regions containing the same SXY module. The Y element binds the heterotrimeric transcription factor NFY. The X region is recognized by the RFX complex, whereas $\mathrm{X} 2$ is bound by CREB. The factor binding to the $\mathrm{S}$ region remains unidentified. Although these transcription factors are ubiquitous and expressed constitutively, they fail to induce genes transcription on their own. Instead they form a multiprotein complex known as the MHC II enhanceosome that binds the SXY module in a stereospecific and cooperative manner, and provides the appropriate interaction surface for the recruitment of CIITA, which is imperative for MHC II genes expression. CIITA interacts with an array of TAFs and elongation factors such as P-TEFb. CIITA also coordinates the recruitment of factors involved in chromatin modification and remodeling including CBP, p300, PCAF, BRG1 and CARM1. Thus, the CIITA production induces or enhances the association of transcription factors with the promoter and activates the synthesis of MHC proteins. 
Factors that activate or inhibit MHC II expression act mainly via the promoters that drive transcription of the MHC2TA gene, which encodes CIITA ${ }^{3,4}$. Similar to MHC II, CIITA transcription is constitutively active in professional APCs, such as dendritic cells (DCs) and B cells, and induced in most non-hematopoietic (or non-immune) cells, notably after interferon (IFN)- $\gamma$ stimulation. This cell type-specific and modulated expression of MHC2TA gene is controlled by the differential activities of the three promoters $\mathrm{pI}, \mathrm{pIII}$ and $\mathrm{pIV}^{20}$.

The different CIITA promoters do not share any sequence homology and are not co-regulated. Each promoter precedes alternative first exons that are spliced to the shared downstream exons, and this gives rise to three types of transcripts (type I, type III and type IV), which only differ at their $5^{\prime}$-ends ${ }^{20}$. As a consequence, three CIITA isoforms that differ at their N-termini are produced (Figure 2). All three protein variants exist in vivo and reside in both the cytoplasmic and nuclear compartments ${ }^{21-23}$, presenting comparable rates of nuclear translocation necessary for transactivation activity $^{23}$. Hence, the CIITA isoforms I, III and IV seem to be functionally interchangeable, being able to activate MHC II genes to equivalent levels when expressed in cells of non-hematopoietic origin ${ }^{3}$. The isoform I is, however, slightly more efficient than the others at activating MHC II promoters, and this particularity has been attributed to the $\mathrm{N}$-terminal sequence that is encoded by the alternative first exon that follows pI, which contains a weak homology to caspase-recruitment domains $(\text { CARDs })^{24}$. Whatever this additional domain confers different functions to the isoform I is still not clear. The isoform III is the best characterized among the three isoforms ${ }^{2}$, since it was the first discovered and has been used in diverse studies involving protein 
knockout or overexpression to investigate the CIITA regulation, activities and also its applicability in immunotherapies ${ }^{25-34}$.

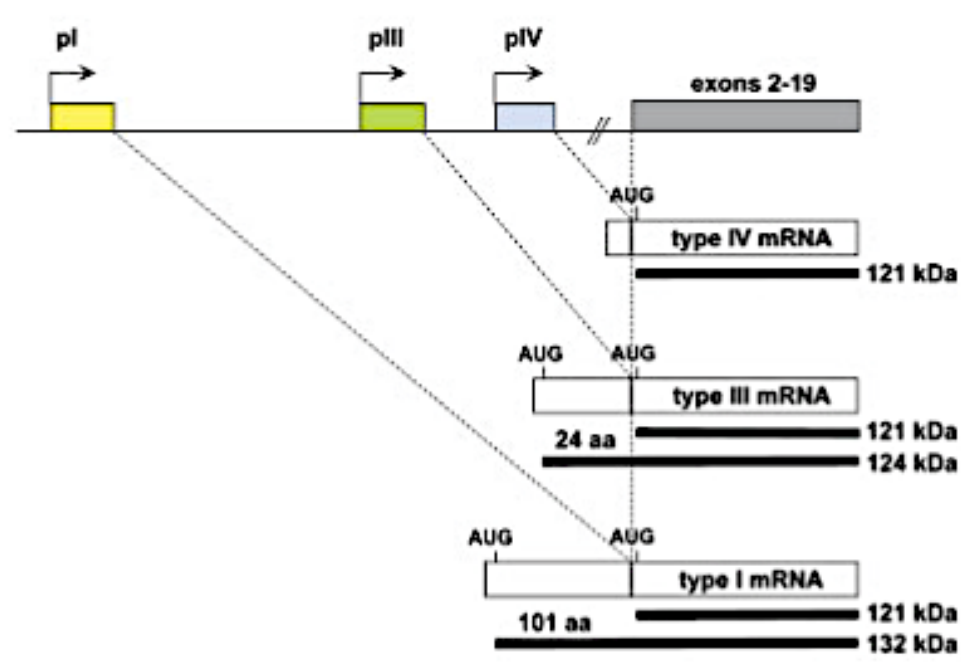

SOURCE: Modified from LeibundGut-Landmann et al., 2004

Figure 2 - Regulatory regions of the gene encoding CIITA. Three independent promoters control the MHC2TA gene transcription (pI, pIII and pIV). Each promoter precedes alternative first exons that are spliced to the shared downstream exons (exons 2-19), so three types of mRNA encoding CIITA (type I, type III and type IV) can be generated. The mRNAs encode distinct protein isoforms of 132, 124 and $121 \mathrm{kDa}$ that differ only in their amino $(\mathrm{N})$ termini. Colored bars represent exons. A vertical line indicates the boundary between the alternative first exon and the shared downstream exons. The positions of translation initiation codons are indicated.

All three isoforms share the majority of CIITA functional regions, which include an acidic activation domain (AD), an acetyltransferase domain (AT), a proline/serine/threonine (PST) domain, a GTP binding domain (GDB) and finally a Cterminal leucine rich region (LRR). The CIITA AD domain binds to general transcription factors and the $\mathrm{CBP}$, leading to the activation of the MHC class II promoter $^{14}$. This domain also partially overlaps the region required for the AT activity of CIITA ${ }^{19}$. The integrity of PST is essential for CIITA function, but its specific role remains unknown ${ }^{36}$. The LRR domain, which interacts with the GDB, is known to play an important role in CIITA movement into the nucleus and in regulating the transactivation function ${ }^{29,36,37}$. The GDB domain, which is the most studied among the 
CIITA domains, has been shown to be the site of interaction of several DNA binding transactivators $^{38}$. The GDB regulates translocation of CIITA: mutation or deletion of this domain results in increased nuclear export, suggesting that GDB is a negative regulator of CIITA nuclear export ${ }^{39}$. Two nuclear localization signals (NLS) have been mapped to the protein $\mathrm{N}$-terminus, as well as an additional one in the $\mathrm{C}$-terminus ${ }^{39}$.

More recently, Soe et al. ${ }^{40}$ described two additional functional domains mapped on the $\mathrm{N}$ - and C-terminals of CIITA, consisting of atypical serine/threonine kinases that have both auto- and trans-phosphorylation activities. The identification of this intrinsic kinase activity, while presenting an AT activity, highlighted the similarity between CIITA and the general transcription factor TAF1 (TATA-binding protein [TBP]associated factor 1). TAF1 is the largest factor in the TFIID complex, which binds to the core promoters and regulates the transcription initiation mediated by the basal transcriptional machinery ${ }^{41}$.

All three CIITA isoforms have remarkable functional parallels with TAF1. Like TAF1, CIITA associates with general transcriptional factors (e.g., TFIIB and P-TEFb) to form a TFIID-like complex ${ }^{15,16,42}$. Both CIITA and TAF1 have AT activity that is essential for transcription ${ }^{19,43,44}$ and both AT activities are negatively regulated by $\mathrm{TAF}^{42,44}$. CIITA and TAF1 both have kinase activity that results in autophosphorylation leading to TAF7 dissociation ${ }^{40,45,46}$. Based on these findings, Devaiah and Singer $^{47}$ suggested that CIITA functions as a general transcription factor and functional homolog of TAF1, independent of its role as a coactivator that nucleates the enhanceosome. This additional CIITA regulatory activity indicates that it can act in non-MHC genes that do not contain the SXY modules and hence do not support enhanceosomes formation, implicating a broader role for CIITA in gene regulation outside of antigen presentation. 
Investigations over the last few decades have greatly expanded the spectrum of target genes that are transcriptionally regulated by CIITA. A transcriptional comparison of CIITA-expressing Raji B cells and the CIITA-deficient RJ2.2.5 cells using cDNA microarrays identified 43 differentially expressed genes ${ }^{48}$. These genes were found to have diverse functions, including antigen processing, intracellular signaling and cell proliferation, suggesting that CIITA may play a general role in the physiology of the cells that are focused on presenting and processing antigens.

Accordingly, a recent ChIP-seq derived genome-wide map for CIITA occupancy in primary human B cells and monocytes, resting and treated with IFN- $\gamma$, identified more than 500 CIITA-bound loci, most of them localized in proximity to identified genes and associated with accessible chromatin ${ }^{49}$. The number and location of CIITA loci varied between B cells and monocytes, and was drastically increased in IFN- $\gamma$ treated monocytes ${ }^{49}$, highlighting the complexity and specificity of the CIITA function across different cell types and conditions.

Among the target genes identified by microarray assays, some considered immunologically relevant were already characterized as genuine targets of the transcriptional factor regulation through the use of CIITA-deficient mice. In DCs, the CIITA positively regulates plexin-A1, which functions in the formation of membrane microdomains on APCs that are optimal for interaction with $\mathrm{T}$ cells ${ }^{50}$. In this same cell type, the interleukin-10 (IL-10) is effectively repressed by CIITA ${ }^{51}$, impairing the induction of $\mathrm{T}$ regulatory cells or suppression of immune response by $\mathrm{DCs}^{52}$. The Fas ligand (FasL) transcription is also repressed by CIITA in $\mathrm{CD}^{+} \mathrm{T}$ cells upon activation $^{53}$, preventing cell death mediated by the Fas:FasL pathway ${ }^{54}$. Activated CD $4^{+}$ $\mathrm{T}$ cells expressing CIITA downregulate IL-4 expression ${ }^{55}$, inhibiting the immune response polarization toward a type $2 \mathrm{~T}$ helper response (Th2). These unraveled down- 
modulatory activities of CIITA have been suggested to be due to its ability to titrate the CBP transcription factor from the promoters/enhancers of the repressed genes ${ }^{51,53,55}$.

Altogether, the findings described above attest to the importance of CIITA as a pluripotent modulator of transcription, which coordinates several aspects of cells physiology and activity in order to primarily promote an effective antigen-specific immune response. This CIITA versatility raises the critical question of how its transcriptional activity is tailored to meet the specific requirements of different cells in distinct physiological and pathological microenvironments. The answer lies in the fact that the ability of CIITA to modulate transcription is itself fine-tuned by a complex layer of regulatory pathways, ranging from promoter selectivity to mRNA stability and posttranslational modification (PTM).

As mentioned before, the cell type-specific and cytokine-induced CIITA expression is primarily determined by transcriptional control of $M H C 2 T A$ gene through the differential usage of $\mathrm{pI}$, pIII and pIV promoters. When those promoters were first discovered, pI was shown to be active mainly in DCs, pIII in B cells, and pIV to be inducible by IFN- $\gamma$. However, it has become clear that the restriction of these promoters to different cell types is not so simple. The three $M H C 2 T A$ promoters were reported to be inducible by IFN- $\gamma^{56,57}$, but they function independently of one another without significant cross-talk ${ }^{3}$. Cells of myeloid origin (DCs and macrophages) rely mainly on pI for constitutive or IFN- $\gamma$-induced CIITA expression ${ }^{20,21,58,59}$. Both pI and pIII were found to be active in $\mathrm{DCs}^{21}$. In cells of lymphoid origin (B cells, $\mathrm{T}$ cells and plasmacytoid DCs) CIITA expression is driven almost exclusively by $\mathrm{pIII}^{2,20,58,60-62}$. Finally, pIV is indispensable for IFN- $\gamma$-activated expression in non-immune cells ${ }^{20,57,63,64}$. 
The activity of each promoter can be regulated by epigenetic mechanisms that allow chromatin remodeling to repress or facilitate the CIITA gene transcription. The pIV promoter, generally inactive in most cell types, is liberated for binding factors and transcription activation, after IFN- $\gamma$ stimulation, through the intense histone acetylation $^{65}$ accompanied by the recruitment of $\mathrm{BRG} 1^{66}$, which is the ATPase subunit of nucleosome remodeling complexes. In trophoblasts, whose the absence of MHC II expression is critical for preventing rejection of the fetus by the maternal immune system, the $\mathrm{pIV}$ is hypermethylated at $\mathrm{CpG}$ dinucleotides to silence CIITA expression $65,67-69$. Also, the pI promoter can be downregulated by histone deacetylation of a large domain that spans the entire regulatory region of the gene $\mathrm{e}^{21,70}$.

When the aforementioned modulatory mechanisms result in active MHC2TA transcription, the final protein levels in the cells may still be altered by posttranscriptional regulation. The transcribed mRNA contains predicted micro-RNA (miRNA) binding sites at the 3' untranslated region (UTR) that can greatly accelerate the transcript degradation and physically impair the mRNA translation ${ }^{71,72}$, decreasing the CIITA synthesis. In addition, once the protein is translated, its half-life can be modulated by mechanisms that interfere with the CIITA stability.

The MHC II transactivator was shown to be a very unstable protein with a short half-life, mainly due to rapid degradation via the ubiquitin-proteasome pathway ${ }^{73}$. The proteasome consists of a $26 \mathrm{~S}$ multi-protein complex comprised of a $19 \mathrm{~S}$ regulatory particle and a $20 \mathrm{~S}$ proteolytic core ${ }^{74}$. The $19 \mathrm{~S}$ particle recognizes the ubiquitin chains on the targeted protein, cleaves the chains, unfolds the protein, and directs the unfolded protein to the $20 \mathrm{~S}$ core for degradation ${ }^{75,76}$. Poly-ubiquitylated intermediaries of CIITA were already detected ${ }^{73,77}$, which are quickly recognized by the $19 \mathrm{~S}$ regulatory particle and subsequently degraded by the $20 \mathrm{~S}$ core. Interestingly, mono-ubiquitylated CIITA is 
not degraded, but instead presents an enhanced transcriptional activity ${ }^{78}$. The ubiquitination is a PTM that involves the covalent transference of a small polypeptyde (ubiquitin) to CIITA. This protein is also subjected to other PTMs including phosphorylation and acetylation/deacetylation, which can accelerate or retard the CIITA degradation by the proteasome as a means of manipulating its activity ${ }^{77,79-81}$. In addition, the interaction with other proteins, such as promyelocytic leukemia (PMLs) proteins, was also suggested to protect CIITA from proteasomal degradation, prolonging its transcriptional activity ${ }^{82}$.

PTMs can also directly interfere with CIITA transcriptional activity by altering its subcellular compartmentalization. Under normal conditions, CIITA is found in both the nucleus and cytoplasm of cells ${ }^{83}$. Since this protein needs to be present in the nucleus to be a potent transcriptional modulator, various PTMs operate to control the subcellular shuttling of CIITA. CBP-pCAF-dependent acetylation of the LRR region facilitates nuclear localization of CIITA ${ }^{84}$, leading to an elevated transactivation of the MHC II gene. More recently, Morgan et al. ${ }^{78}$ revealed that CIITA ubiquitination specifically at the lysine 63 is required for the following extracellular signal regulated kinase (ERK)1/2-mediated phosphorylation, which is necessary for the CIITA nuclear localization. This study was the first to demonstrate a crosstalk between CIITA ubiquitination and phosphorylation to regulate protein location and activity, highlighting the importance of evaluating the combined effect of PTMs in the CIITA functionality.

Finally, PTMs can alter interactions of CIITA with other transcription factors as an additional mechanism to fine-tune the transactivation activity. For instance, increased overall phosphorylation by protein kinase A (PKA) promotes CIITA interaction with the coactivator $\mathrm{p} 300^{31}$. Ubiquitinated CIITA preferentially interacts 
with RFX5 and NFY, enhancing the activated MHC II transcription ${ }^{85}$. Contrarily, the deacetylation mediated by histone deacetylase 2 (HDAC2) disrupts the interaction of CIITA with RFX5, reduces the recruitment of CIITA to target promoters, and consequently suppresses its transcriptional activity ${ }^{79}$.

Besides the wealth of knowledge about the different mechanisms regulating CIITA, the vast majority of studies evaluated the outcome of individual mechanisms in the factor activity. Therefore, the understanding of the combined effect of these several layers of regulatory pathways on CIITA expression and activity in different cell types is still incipient. Since this transcription factor was first described as the master regulator of antigen presentation, professional APCs have been extensively used in CIITA studies. The knowledge obtained thus far enables the generation of a better overall view of the CIITA expression and activity patterns in these cell types.

In general, the professional APCs constitutively express the CIITA protein until facing maturation stimuli, provided by the encounter of an antigen along with inflammatory conditions, which lead to the downregulation of CIITA activity. Dendritic cells located in the peripheral tissues constitutively express both isoforms I and III, with the isoform I being predominantly produced ${ }^{21}$. Upon maturation, these cells initiate a migration to the lymph nodes while blocking the MHC2TA transcription through the histone deacetylation of a large regulatory region of promoter $\mathrm{pI}^{21,70}$. In parallel, both isoforms activities are impaired by their enhanced nuclear export ${ }^{86}$. Similarly, the isoform I production by macrophages is drastically hampered by maturation stimuli through the increased mRNA degradation mediated by miRNA binding $^{20}$. An MHC2TA switch off also accompanies the terminal differentiation of $\mathrm{B}$ cells into plasma cells. This repression has been proposed to be promoted by binding of 
the transcriptional repressor B-lymphocyte-induced maturation protein 1 (BLIMP1) to a site in the pIII promoter ${ }^{87,88}$.

This common pattern of CIITA silencing is known to be critical at least for DCs functions. When those cells are in the periphery capturing antigens, the CIITA expression allows an intense de novo synthesis of MHC II molecules which are partially kept in intracellular vesicles ${ }^{21,89}$. Once they capture an antigen in the presence of inflammatory stimuli, the levels of MHC II molecules expressed at the cell surface is increased as a result of changes in the intracellular localization and stability of preexisting MHC II proteins ${ }^{90}$. In contrast, de novo synthesis of these molecules is shut down by CIITA silencing ${ }^{21,70}$. These processes promote cell-surface retention of peptide-MHC II complexes presenting antigens captured prior to the DC maturation and migration to lymph nodes, ensuring that MHC II-restricted antigen presentation to $\mathrm{CD}^{+}$ $\mathrm{T}$ cells focuses on foreign antigens and not on endogenously synthesized autologous proteins $^{70}$. Since macrophages and B cells also present CIITA silencing mechanisms activated by maturation stimuli, the dynamics of CIITA and MHC II expression revealed in DCs is likely to be shared among professional APCs as a way to guarantee the proper activation of antigen-specific $\mathrm{CD}^{+} \mathrm{T}$ cells.

Different from the professional APCs, non-immune cells are generally unable to present antigens to $\mathrm{CD}^{+} \mathrm{T}$ cells due to the lack of MHC II molecules expression. Under normal conditions, the $M H C 2 T A$ promoters are maintained inactive by epigenetic mechanisms $^{65}$ and thus these cells express only MHC I molecules, which present endogenously synthesized antigens to $\mathrm{CD}^{+} \mathrm{T}$ cells. However, it is well known that most non-immune cells can express MHC II molecules and become non-professional APCs under IFN- $\gamma$ stimulation $^{20,57,63,64,91,92}$, which acts through the Janus kinase (JAK)signal transducer and activator of transcription (STAT) pathway to activate the pIV 
promoter and the CIITA transcription ${ }^{63}$. This induced activation is generally a transient event, since the IFN- $\gamma$ also induces the suppressor of cytokine signaling-1 (SOCS-1) that acts as a feedback inhibitor of IFN- $\gamma$-activated $\mathrm{pIV}^{93}$. However, the mechanisms by which non-immune cells can extend or shorten the CIITA expression and activity at the post-transcriptional level are still poorly understood.

Since the majority of regulatory mechanisms were described in professional APCs, it remains unclear whether they are also active in non-immune cells and even if distinct types of non-immune cells can differentially coordinate the complex layers of CIITA regulation to enable tissue-specific CIITA activity. In light of the broad regulatory activity of CIITA beyond the MHC II genes expression, the understanding of how different non-immune cells regulate this factor will be fundamental for the establishment of CIITA tissue-specific functions and its involvement in the control of immune response at the peripheral tissues.

The discovery that most non-immune cells can express MHC II molecules through the IFN- $\gamma$-induced CIITA expression shedded light on the possibility to develop cell-based cancer vaccines by obtaining IFN- $\gamma$-treated tumor cells that can act as "surrogate APCs" for tumor-associated antigens and thus facilitate tumor rejection ${ }^{94,95}$. Those surrogate APCs obtained in vitro would present peptides on their surface derived from endogenously synthesized tumor antigens in the context of MHC II molecules, and once reintroduced into the organism bearing the cell-derived tumor, would be able to activate antigen-specific $\mathrm{CD}^{+} \mathrm{T}$ cells help to potentiate the anti-tumor $\mathrm{CD}^{+} \mathrm{T}$ cell cytotoxic activity (CTL). However, this initial immunotherapeutic approach was limited by the aforementioned IFN- $\gamma$ induction of a negative feedback that restrains the CIITA expression, resulting in a transient MHC II production ${ }^{93}$, together with the fact that 
some tumor cells, such as human hepatocarcinoma and pancreatic adenocarcinomas, were shown to be refractory to IFN- $\gamma$ induction ${ }^{96-98}$.

These drawbacks were circumvented by the substitution of IFN- $\gamma$ treatment for exogenous CIITA expression in tumor cells. First, Chang et al. ${ }^{27,99}$ showed that the transfection of non-immune cells with CIITA cDNA was sufficient for the induction of MHC II genes, similarly to IFN- $\gamma$-treated cells. Second, transfection of CIITA cDNA under the control of a ubiquitous promoter in MHC II-inducible cells resulted in a constitutive class II expression ${ }^{99-101}$, preferable to the IFN- $\gamma$-inducible transient expression. Lastly, IFN- $\gamma$ refractory tumors were shown to present inhibitory mechanisms acting at the CIITA transcriptional level ${ }^{97,98}$, since the CIITA transfection was able to restore not only MHC II expression but also the tumor cells capacity of processing and presenting antigens to antigen-specific $\mathrm{CD}^{+} \mathrm{T}$ cells in vitro ${ }^{97}$. Taken together, these findings endorsed the extensive application of a CIITA exogenous expression in the development of cell-based cancer vaccines ${ }^{28,97,102-108}$.

The greatest advantage of this cancer immunotherapeutic approach is the potential to present a diversity of immunogenic peptides endogenously produced by the tumor cells. Even though the MHC II molecules are known to preferentially present peptides derived from exogenous antigens, CIITA transfectants were shown to efficiently present the endogenous tumor antigens to $\mathrm{CD}^{+} \mathrm{T}$ cells ${ }^{28,106-108}$. As mentioned before, the CIITA expression induces not only MHC II molecules but also accessory proteins including the invariant chain (Ii). Ii is known to hinder the presentation of endogenously synthesized peptides and favors the presentation of antigens acquired by endocytosis. Ii mediates this effect by binding to newly synthesized MHC II molecules in the endoplasmic reticulum and preventing them from acquiring peptides of endogenously synthesized molecules ${ }^{109}$. Considering the potential 
negative effect of Ii coexpression in the tumor antigens presentation, few vaccine strategies added the Ii silencing by small interfering RNA (siRNA) to the CIITA $\operatorname{transfection}^{104,105}$. However, Thompson et al. ${ }^{102}$ elegantly demonstrated that CIITA transfectants with or without the Ii siRNA are equally efficient at activating antitumor $\mathrm{CD}^{+} \mathrm{T}$ cells, indicating that Ii does not impair endogenous antigen presentation.

Accordingly, CIITA-transfected tumor cells evaluated in experimental models of immunizations have shown that the CIITA-dependent MHC II expression confers to tumor cells the capacity to act as a protective vaccine. The first example of successful tumor vaccination was reported by Meazza et al. ${ }^{28}$, using a very malignant murine mammary adenocarcinoma cell line (TS/A) transfected with the isoform III of human CIITA. These cells were fully rejected by $51 \%$ of syngeneic recipients and had a significant lower growth rate in the remaining animals. Tumor rejecting animals were protected against challenge with the parental tumor, and this protection required both $\mathrm{CD}^{+}$and $\mathrm{CD}^{+} \mathrm{T}$ cells, with $\mathrm{CD} 4^{+} \mathrm{T}$ cells being fundamental in the priming phase of the antitumor response.

Further investigations showed that the tumor-specific $\mathrm{CD}^{+} \mathrm{T}$ cells activated by the surrogate APCs had the functional characteristics of Th1 cells and produced IFN- $\gamma$, which was important for the generation of an antitumor response and establishment of immune memory ${ }^{106}$. Interestingly, vaccinated mice presented a statistically significant reduction in the percentage and absolute number of $\mathrm{CD} 4^{+} \mathrm{CD} 25^{+} \mathrm{T}$ regulatory cells (Treg) as compared with those of untreated mice with growing tumors and mice vaccinated with the non-transfected parental TS/A cells ${ }^{107}$. Although the mentioned studies did not evaluate the influence of CIITA expression in non-MHC II genes, the Th1 polarization together with the reduction of Tregs is in accordance with the CIITAmediated downregulation of IL-10 and IL-4 expression mentioned before. This indicates 
that the exogenous CIITA expression in non-immune cells leads to modifications that extend beyond control of MHC II genes, generating surrogate APCs able not only to present antigens to $\mathrm{CD} 4^{+} \mathrm{T}$ cells but also to modulate the generated $\mathrm{T}$ helper response.

The general application of this cell-based cancer vaccine model was established by verifying a similar activation of a protective immune response using tumors of distinct histological origin including colon adenocarcinoma, renal adenocarcinoma and sarcoma, all transfected with the isoform III of human CIITA $^{108}$. Mice immunized with different tumor cells expressing CIITA showed no signs of autoimmunity, indicating that most of the stimulated immune response was directed against tumor antigens, and not self antigens ${ }^{108}$. Importantly, all three transfected tumor cells were able to induce tumor rejection and/or retardation of growth, but each cell type presented specific stability of CIITA expression resulting in different levels of MHC II expression that correlated with the capacity to reject the tumor.

Taking into account that the three cell types were transfected with the same CIITA DNA under the control of a constitutive promoter, the differential CIITA stability demonstrated by Frangione et al. ${ }^{108}$ probably reflects the interference of posttranscriptional regulatory mechanisms acting in a cell-type specific manner that were not investigated in the mentioned study. Nevertheless, the demonstration that the CIITA genetic transfer is able to transform different cell types into efficient surrogate APCs, together with the previous demonstration that these surrogate APCs are able to activate and modulate the $\mathrm{CD}^{+} \mathrm{T}$ helper response, disclosed the possibility to use the exogenous CIITA expression as an adjuvant strategy for vaccines and immunotherapies.

To date, the CIITA application has been mainly restricted to the development of antitumor vaccines. However, this transcriptional factor can potentially be used as a molecular adjuvant for the improvement of different immunotherapeutic approaches. 
For instance, CIITA could be applied to genetic immunizations, such as DNA and viral vector vaccinations, in which the combined delivery of antigen and CIITA encoding sequences would improve the immunogenicity of in vivo transfected immune cells and also transform any transfected non-immune cells into surrogate APCs. This strategy would thus amplify the vaccine capacity to activate antigen-specific $\mathrm{CD} 4^{+}$helper $\mathrm{T}$ cells and therefore elicit a more robust immune response. Additionally, the CIITA adjuvant effect could be explored to improve dendritic cells-based immunotherapies, in which the CIITA overexpression by the in vitro generated dendritic cells would intensify their immunogenic potential.

Dendritic cells are the most potent APC and can play a critical role in the initiation of a $\mathrm{T}$ cell-mediated immunity in vivo ${ }^{110,111}$. These cells are responsible for triggering and modulating the immune response against invading pathogens and certain malignant cells ${ }^{112}$, and one key factor determining the effectiveness of the immune response is the maturation status of the $\mathrm{DCs}^{113}$. In the absence of danger signals, the immature DCs (iDCs) expressing few MHC II and co-stimulatory molecules have high capability of antigen capture and processing. In contrast, danger signals induce maturation of DCs (mDCs), which present a decreased capacity to process antigens, but express higher levels of T cell-activating molecules, including MHC II, CD80, CD86, CD40 and CD83, and secrete proinflamatory cytokines, thus effectively priming $\mathrm{T}$ cell responses $^{110,112}$.

Due to the critical role of this professional APC in the immune response, DCs generated in vitro from monocyte precursors and loaded with antigen have been intensely studied as a tool to treat a variety of cancers and Human Immunodeficiency Virus (HIV) disease ${ }^{114-117}$. The in vitro generation of dendritic cells is achieved through culturing monocytes isolated from the peripheral blood of patients in the presence of 
granulocyte macrophage colony-stimulating factor (GM-CSF) and IL-4 to obtain iDCs. The maturation of these cells prior to their use in immunotherapies is one of the most critical parameters for the activation of an effective immune response, and can be induced by a plethora of methods that result in the obtainment of mDCs with striking functional differences.

Dendritic cells maturing under different conditions can instruct naïve $\mathrm{CD} 4^{+} \mathrm{T}$ cells to selectively acquire Th1 or Th2 effector functions, or even promote $\mathrm{T}$ cell activation without polarizing the $\mathrm{T}$ helper response ${ }^{118-121}$. Depending on the type of effector response they induce, the mDCs can be classified into non-polarized, type-1- or type-2-polarized mDCs, here referred to as DC-0, DC-1 and DC-2, respectively. Multiple research groups have developed different maturation protocols for generating each mDC subtype ${ }^{122-129}$ and also intermediary mDCs, which present mixed features from different subtypes ${ }^{119}$. For instance, DC-0 can be obtained by incubating iDCs with CD40L alone $\mathrm{e}^{121}$, or with a cytokine cocktail containing IL-1 $\beta$, tumor necrosis factors (TNF)- $\alpha$ and IL-6 ${ }^{130}$; DC-2 can be matured in the presence of Prostaglandin $\mathrm{E}_{2}\left(\mathrm{PGE}_{2}\right)$, along with IL-1 $\beta$ and TNF- $\alpha^{130-132}$; and the most potent DC-1 $(\alpha \mathrm{DC} 1)$ is matured in the presence of IFNs and Toll-like receptor (TLR) ligands, including IL-1 $\beta$, TNF- $\alpha$, polyinosinic:polycytidylic acid (Poly-I:C), IFN- $\alpha$ and IFN- $\gamma^{129}$.

Interestingly, these mDC subtypes express similar levels of MHC II, CD83, CD86 and C-C chemokine receptor 7 (CCR7), indicating a similar state of surface phenotypic maturation ${ }^{130}$. In contrast, once activated by the interaction with CD40L present on the surface of $\mathrm{CD} 4^{+} \mathrm{T}$ helper cells, each subtype present a distinctive level of IL-12(p70) secretion, the major Th1-driving cytokine ${ }^{133}$. In this regard, DC-2 is the least efficient in this cytokine production, DC-0 secretes intermediary levels and DC-1 presents the highest IL-12(p70) production (around 15-fold superior to DC-2) ${ }^{129}$. This 
pattern is directly correlated with each subtype polarizing activity, since DC-1 exhibits a superior ability to induce a Th1 response. In fact, Watchmaker et al. ${ }^{134}$ already demonstrated that naïve $\mathrm{CD}^{+} \mathrm{T}$ cells activated by DC-2 did not acquire CTL functions and remained responsive to lymph node-associated chemokines, even though they vigorously expanded. On the other hand, the naïve $\mathrm{CD}^{+} \mathrm{T}$ cells activated by $\mathrm{DC}-1$, specifically the $\alpha \mathrm{DC} 1$, presented much higher proliferation, CTL functions and migration to the target sites ${ }^{134}$.

Taking into account the plasticity of DCs and their ability to adopt different functions, it is possible to match the desired mDC subtype to their clinical application. Therefore, DC-2 cells may be used for immunotherapies against intestinal parasites, allergies and autoimmune diseases, inducing $\mathrm{T}$ cells to produce IL-4 and IL-5 and promoting antibody production. Although DC-0 and even DC-2 cells have been applied in immunotherapies against cancers ${ }^{135-137}$ and HIV infection ${ }^{115,116,138-143}$, DC-1 is currently considered the most effective subtype to be used in these clinical applications, since only DC- 1 is able to induce $\mathrm{CD}^{+} \mathrm{T}$ cells producers of IFN- $\gamma$ and lymphotoxin, and CD ${ }^{+}$CTLs capable of effectively eliminating tumor cells and fighting the HIV infection $^{144-147}$.

In regards to DC-based cancer immunotherapy, despite the demonstration that the antitumor vaccination protocols are able to modify the natural history of the disease, the preliminary results obtained with clinical trials have been inconsistent and with limited clinical responses ${ }^{148}$. These apparent DC vaccinations failures have been partially ascribed to the recent finding that monocyte-derived (mo-)DCs from cancer patients show an altered phenotype and preferentially induce Tregs, a phenomenon that occurred regardless of the $\mathrm{mDC}$ subtype induced ${ }^{149}$. Since the vaccination protocols utilize DCs derived from autologous monocytes, it was suggested that patients' tumors 
are able to program monocytes to be differentiated into DCs producing IL-10 and TGF$\beta$, features that cannot be altered in vitro by maturation cocktails. Similarly, a functional bias to induce immunosuppressive responses has been demonstrated in DC-based immunotherapies against HIV-1 $1^{150,151}$.

Our research group headed by Prof. Alberto Duarte (Laboratory of Dermatology and Immunodeficiency - LIM-56, University of São Paulo Medical School, Brazil) currently runs a phase I/II clinical trial involving chronically infected HIV-1-positive individuals immunized with DCs pulsed with the whole autologous virus, previously inactivated by aldrithiol-2 (AT-2). The precedent study evaluating this vaccine efficacy showed that eight out of 18 untreated HIV-1-infected individuals presented prolonged suppression of viral load of more than $90 \%$, positively correlated with HIV-1-specific activated $\mathrm{CD}^{+} \mathrm{T}$ cells and with HIV gag-specific perforin-expressing $\mathrm{CD} 8^{+}$effector cells $^{115}$. Although the phase I/II clinical trial follow-up is still under evaluation and no statistically relevant data is available, mDCs derived from nine patients, pulsed with autologous inactivated virus and co-cultured with autologous lymphocytes, were capable of inducing IL-2 and IFN- $\gamma$-expressing $\mathrm{CD}^{+} \mathrm{T}$ cells but elicited a weak cytotoxic activity of $\mathrm{CD}^{+} \mathrm{T}$ lymphocytes (unpublished data). These preliminary data indicate that the immunogenic potential of this vaccine may be limited by the previously shown functional deviation of DCs triggered by the HIV-1 envelope protein gp $120^{150,151}$.

Mo-DCs pulsed with AT-2-inactivated virus or the gp120 protein show enhanced IL-10 production $^{151}$, and an also markedly reduced capacity to produce IL-12 and to induce $\mathrm{T}$ cell proliferation ${ }^{150}$. This induced suppression of DCs activities occurs regardless of the maturation cocktail and does not completely impair the upregulation of MHC II and co-stimulatory molecules indicative of a functional maturation ${ }^{150,151}$. Thus, 
the DC functional deviation may often go unnoticed by the vaccine quality control that relies basically on the evaluation of DCs surface phenotypic markers to assure cells maturation. In addition, the high phenotypical variability between mDCs from different HIV-positive patients, including considerable standard deviations in the levels of MHC II expression, impairs the vaccine formulation standardization ${ }^{152}$.

Therefore, the need for a strategy to bypass this functional deviation and consistently obtain mature and functionally effective mo-DCs from HIV-positive individuals is evident. The use of CIITA as a molecular adjuvant would be particularly interesting due to its pluripotent modulatory activity that can interfere with DC functions in various immunologically relevant aspects. Our rationale is that the CIITA overexpression by DCs would decrease the variability in the MHC II expression and also counteract the HIV-1 envelope-mediated suppressive activity by inhibiting IL-10 expression. Furthermore, the upregulation of plexin-A1 would help improve $\mathrm{T}$ cell activation through a more efficient formation of immunological synapses. Since this approach would also benefit DC-based cancer immunotherapies, the development of strategies to enable the application of CIITA as a molecular adjuvant would consist in an important step toward the improvement of DC vaccines developed in Brazil and worldwide.

Although promising, the use of CIITA as a molecular adjuvant is still incipient, inciting us to explore its impact on the potency of different immunotherapies, including DC-based vaccines and also genetic immunizations. Together, these therapeutic approaches target a wide variety of cell types, ranging from professional APCs to a myriad of non-immune cells, which present very distinct and not completely unraveled transcriptional and post-transcriptional regulatory mechanisms to control CIITA expression. Therefore, in the present study, we intended to develop and validate CIITA 
delivery systems capable of counteracting the known cell-specific regulatory mechanisms and promote a high-efficiency CIITA expression in a broad range of human cell types. Additionally, in the face of the poorly understood regulatory mechanisms controlling the CIITA expression and activity in non-immune cells, we also aimed to apply such delivery systems to investigate the mechanisms of posttranslational regulation of CIITA in these cells and their implications in the use of CIITA as a molecular adjuvant. We believe that the gene delivery tools produced here will enable future studies aiming to thoroughly investigate the CIITA expression effect on the immunogenicity of DC vaccines and genetic immunizations, and the knowledge generated here may greatly contribute to the understanding of the intricate cell typespecific regulation of the MHC II transactivator. 
2 OBJECTIVES 


\subsection{General Objectives}

To develop and validate CIITA delivery systems capable of promoting a highefficient expression of the transcriptionally active CIITA protein in a broad range of non-immune human cell types and in monocyte-derived dendritic cells. In addition, we intended to apply such delivery systems to investigate the mechanisms of posttranslational regulation of CIITA in non-immune human cells.

\subsection{Specific Objectives}

- To in silico design the human CIITA DNA sequence for efficient expression in different human cell types;

- To obtain a DNA plasmid for the designed CIITA sequence delivery;

- To validate the exogenous CIITA expression and nuclear localization in different non-immune human cell lines;

- To validate the exogenous CIITA transcriptional activity through the detection of MHC II molecules expression in transfected non-immune cell lines;

- To compare the exogenous CIITA expression and transcriptional activity between human non-immune cell lines;

- To compare the exogenous CIITA degradation and the influence of proteasomal activity between human non-immune cell lines;

- To investigate the mechanism by which the non-immune cells differentially regulate the CIITA proteasomal degradation;

- To establish the differentiation of human monocytes into immature DC and mature DC-0, DC-1 and DC-2; 
- To establish the efficient CIITA optimized sequence delivery to the primary human dendritic cells;

- To evaluate the phenotypic alterations induced in human dendritic cells derived from healthy individuals and chronically infected HIV-1 patients by the method of CIITA sequence delivery;

- To validate the exogenous CIITA expression and transcriptional activity in human dendritic cells derived from healthy individuals and chronically infected HIV-1 patients. 
3 MATERIALS AND METHODS 


\subsection{Cell Cultures}

HEK293 human embryonic kidney cells, HeLa human cervical carcinoma cells and NIH/3T3 mouse embryonic fibroblast cells (ATCC, Manassas, VA) were grown in Dulbecco`s modified Eagle`s medium (DMEM - Invitrogen, Eugene, OR) supplemented with $10 \%$ fetal bovine serum (FBS; Thermo Scientific, Waltham, MA), 1\% penicillin/streptomycin (Gibco) and 1\% L-glutamine (Sigma-Aldrich). The Huh-7 hepatocellular carcinoma cells (Japanese Collection Research Bioresources, Osaka, Japan), was grown in DMEM supplemented with $10 \%$ FBS, $1 \%$ penicillin/streptomycin, 1\% L-glutamine, 1\% non-essential amino acids (Gibco) and 1\% sodium pyruvate (Gibco). 293T cells were grown in DMEM supplemented with $10 \%$ FBS, 1\% penicillin/streptomycin, 20mM HEPES (Gibco) and 1\% sodium pyruvate. All cell lines were cultured at $37{ }^{\circ} \mathrm{C}$ in $5 \% \mathrm{CO}_{2}$.

\subsection{Subjects}

Peripheral blood mononuclear cells (PBMCs) were purchased from the Western Pennsylvania Blood Bank (Pittsburgh, PA, USA) in the form of buffy coat from four healthy individuals. Cryopreserved monocytes were obtained from buffy coats of three individuals chronically infected with HIV-1 recruited from "Hospital das Clínicas" (University of São Paulo, Brazil). The three participants were over 18 years of age, had $\mathrm{CD}^{+} \mathrm{T}$ cell count $>500$ cells $/ \mu \mathrm{L}$ and were "naïve" for treatment. Written informed consent was obtained according to the protocol of "Hospital das Clínicas" Ethical Committee (CaPPesq - São Paulo, Brazil). 


\subsection{Monocytes isolation and cryopreservation}

Fresh PBMCs from buffy coats were purified using Ficoll-hypaque gradient (GE Healthcare, Little Chalfont, Buckinghamshire, United Kingdom). The collected whole blood was diluted in RPMI-1640 medium (Gibco) at a 1:1 ratio and transferred to 50 $\mathrm{mL}$ conical plastic tubes containing $10 \mathrm{~mL}$ of Ficoll/Hypaque (GE Healthcare). The cells suspension was centrifuged at $975 \mathrm{X} g$ for 20 minutes at room temperature (RT). The resulting PBMC layer was aspirated and washed twice with $10 \mathrm{~mL}$ of RPMI-1640 followed by centrifugation at $450 \mathrm{X} g$ for 10 minutes at RT. Isolated PBMCs were resuspended in $50 \mathrm{~mL}$ of $\mathrm{AIM}-\mathrm{V}$ medium (Gibco) and kept at RT for 16 hours. Then the cells were centrifuged at $450 \mathrm{X} g$ for 8 minutes at RT and the pellet resuspended in $15 \mathrm{~mL}$ of $60 \%(\mathrm{v} / \mathrm{v})$ Percoll (in Iscove`s Dubecco`s Modified Medium (IMDM) Invitrogen). The suspension was aliquoted into six $15 \mathrm{~mL}$ conical tubes and $5 \mathrm{~mL}$ of 46\% Percoll/IMDM solution was carefully added to each aliquot, followed by an additional $5 \mathrm{~mL}$ of $34 \%$ Percoll/IMDM solution, resulting in the formation of three layers per tube. The samples were centrifuged at 2,200 X $g$ for $45 \mathrm{~min}$ at $\mathrm{RT}$ and the monocytes layer obtained in each tube (top layer) was transferred to a single $50 \mathrm{~mL}$ conical tube. After two washes with $50 \mathrm{~mL}$ of RPMI-1640 followed by centrifugation at $450 \mathrm{X} \mathrm{g}$ for $8 \mathrm{~min}$ at $\mathrm{RT}$, the monocytes pellet was resuspended at a concentration of $10^{7}$ cells/mL of FBS $10 \%$ dimethyl sulfoxide (DMSO; Sigma-Aldrich), dripped slowly under ice bath. Cells suspensions were distributed to cryotubes, stored at $-80{ }^{\circ} \mathrm{C}$ and transferred to liquid nitrogen after 24 hours. 


\subsection{Generation of monocyte-derived dendritic cells}

Cryopreserved monocytes were quickly thawed by immersion in a $37^{\circ} \mathrm{C}$ water bath and washed twice with RPMI-1640 at $450 \mathrm{Xg}$ for 8 min at $4{ }^{\circ} \mathrm{C}$. After the second wash, the cells were quantified and resuspended at a concentration of $5 \times 10^{5}$ cells $/ \mathrm{mL}$ in RPMI-1640. The cells were then transferred to a 24 -well plate $\left(5 \times 10^{5}\right.$ cells per well) and incubated for two hours at $37{ }^{\circ} \mathrm{C}$ and $5 \% \mathrm{CO}_{2}$ allowing the monocytes to adhere to the plastic plate. Subsequently, non-adhered cells were washed off the plate and $1 \mathrm{~mL}$ of IMDM 10\% FBS was added to each well, supplemented with recombinant human (rhu) GM-CSF (Sanofi-Aventis, Bridgewater, NJ) and IL-4 (R\&D Systems, Minneapolis, MN), both at 1,000 International Units (IU)/mL. The purified monocytes were cultured during five days for differentiation into immature dendritic cells, which were either kept immature or matured into four polarizing subtypes by cultivation for 48 hours after the addition of the following cytokines cocktails to the culture: (i) DC-0 - 4 ng/mL CD40L (Life Technologies, Grand Island, NY); (ii) DC-2 - 10 $0^{-6} \mathrm{~mol} / \mathrm{mL}$ PGE2 (Sigma-Aldrich), $25 \mathrm{ng} / \mathrm{mL}$ IL-1 $\beta$ and $50 \mathrm{ng} / \mathrm{mL}$ TNF- $\alpha$ (R\&D Systems); (iii) DC-1 50 ng/mL TNF- $\alpha, 25$ ng/mL IL-1 $\beta$ (R\&D Systems), $20 \mu \mathrm{g} / \mathrm{mL}$ Poly-I:C (SigmaAldrich), 1,000 IU/mL IFN- $\alpha$ and 1,000 IU/mL IFN- $\gamma$ (Miltenyi Biotec, San Diego, CA). On the seventh day of culture, iDCs and mature DC subtypes (mDCs) were removed from the plate through gentle pipetting with cold phosphate-buffered saline (PBS) to be used in subsequent assays. 


\subsection{Genetic Sequence Optimization}

The amino acid sequences encoding the full-length isoform III of the human CIITA protein (access number: NP_000237.2) and the foot-and-mouth disease virus (FMDV) $2 \mathrm{~A}$ oligopeptide ${ }^{153}$ were reverse translated into DNA sequences using the LETO 1.0 software (Entelechon, Regensburg, Germany). The 2A DNA was fused inframe with the 3' end of CIITA DNA to obtain the CIITA_2A sequence, which was flanked by the Kozak sequence and two translational stop codons. This in silicodesigned sequence was optimized for enhanced mRNA transcription, RNA stability and translation in human cells using the genetic algorithm from the LETO 1.0 software. This software uses genetic algorithms that allow the design of synthetic DNA sequences by means of silent point mutations that alter several genetic features such as codon usage, mRNA secondary structure, GC content distribution, repetitive DNA motifs, restriction sites and cryptic splice sites, among others. All of these parameters are considered simultaneously, in order to significantly increase antigen expression in human cells. The final OPT CIITA_2A DNA was commercially synthesized (GeneScript, Piscataway, NJ). The CIITA fusion with 2A will allow the future development of bicistronic vectors controlling the expression of CIITA together with a sequence-of-interest by a unique promoter.

\subsection{Plasmids and cloning strategies}

The initial validation of the designed CIITA sequence performed in the present study utilized monocistronic plasmids encoding only the CIITA optimized sequence. 
Thus, PCR amplifications were performed to obtain CIITA amplicons, lacking the 2A sequence, with inserted cleavage sites that facilitated cloning strategies. PCR assays were carried out using the GoTaq Flexi DNA polymerase (Promega, Madison, WI) and $0.5 \mu \mathrm{M}$ of each primer. Amplified products were analyzed by electrophoresis in a $1 \%$ agarose gel. The sequences of primers utilized for each cloning strategy are described in Table 1.

Table 1 - Sequences of primers used in PCR reactions

\begin{tabular}{|c|c|c|}
\hline Constructs $^{1}$ & Forward & Reverse \\
\hline $\mathrm{p} / \mathrm{CIITA}$ & TGACTAGTACCATGGGGTGTCTCGCACCC & GAGGTACCTTATCACCTCAGGCTGATCC \\
\hline pMACS-CIITA & TGCGTACGACCATGGGGTGTCTCGCACCC & GGCCGCGGCCGCTTATCACCTCAGGCTGATCC \\
\hline pMACS-eGFP & TGCGTACGACCATGGTGAGCAAGGGCGAGGAGC & GCATGCGGCCGCTTATCAACTACTTGTACAGCTCGTCCATGCCGA \\
\hline SiEW-CIITA & TGTGCCGCGGCCGCGGACCATGGGGTGTCTCGCACCC & GAGACCGCGGCCGCGGTTATCACCTCAGGCTGATCC \\
\hline L/CIITA & TGGAATTCACCATGGGGTGTCTCGCACCC & GGCCGCGGCCGCTTATCACCTCAGGCTGATCC \\
\hline L/eGFP2 & TGGCTAGCACCATGGTGAGCAAGGGCGAGGAGC & GCATGCGGCCGCTTATCAACTACTTGTACAGCTCGTCCATGCCGA \\
\hline
\end{tabular}

The CIITA amplicon was inserted into the p43.2 vector between the SpeI and KpnI cleavage sites to generate the p/CIITA construct. The expression from this vector is controlled by a cytomegalovirus (CMV) enhancer-promoter. The pMACS plasmid was acquired as part of the magnetic separation kit MACSelect System (Miltenyi Biotec). This bicistronic expression vector contains the T7 RNA polymerase promoter upstream from a multiple cloning site (MCS), followed by an internal ribosome entry site (IRES) and the truncated human low affinity nerve growth factor receptor (LNGFR) cDNA. When a gene-of-interest is cloned into the MCS, a bicistronic mRNA is transcribed, which encodes the gene-of-interest as well as the truncated LNGFR molecule. Inserting the CIITA sequence between the BsiWI and NotI cleavage sites in the pMACS-LNGFR MCS generated the pMACS-CIITA construct. The eukaryotic 
expression vector pcDNAl-eGFP, kindly provided by Dr. Jared Evans (Johns Hopkins University, Baltimore, MD, USA), was used as a template for PCR amplification of the eGFP sequence flanked by BsiWI and NotI cleavage sites, which were used for the pMACS-eGFP construct production.

Each aforementioned plasmid was expanded after the transformation of DH5 $\alpha$ Escherichia coli strain (Invitrogen) and purified according to the manufacturer's recommendations for free endotoxin columns (Qiagen, Hilden, Germany).

For the production of lentiviral vector particles, the optimized CIITA sequence was initially cloned into the transfer vector SiEW, kindly provided by Dr. Manuel Grez (Chemotherapeutisches Forschungsinstitut Georg-Speyer-Haus, Germany). The use of SiEW transfer vector allows the production of replicating-incompetent viral particles that are self-inactivated after transduction into target cells. This bicistronic vector presents the spleen focus-forming virus (SFFV) enhancer-promoter controlling the gene-of-interest expression together with eGFP inserted after an IRES site. The SiEWCIITA construct was obtained by inserting the CIITA sequence into the SacII site, the only cleavage site for cDNA cloning in SiEW.

A second lentivector named L/CIITA was created by the insertion of CIITA amplicon into the LeGO-iG2 vector (Addgene, Cambridge, MA). Similar to the SiEWCIITA, L/CIITA is an IRES-containing bicistronic vector for expressing CIITA together with eGFP under the control of the SFFV enhancer-promoter ${ }^{154}$. The LeGO-iG2 vector does not encode any viral protein but contains cis-elements that promote the packing, transcription and integration into the transfected cell genome. These features ensure biosafety and allow the cloning of inserts up to $9 \mathrm{~kb}$ in size. For the production of L/CIITA, the CIITA amplicon was inserted between the EcoRI and NotI cleavage sites of LeGO-iG2. The vector pcDNAl-eGFP was also used as a template for PCR 
amplification of the eGFP sequence flanked by NheI and NotI cleavage sites, which were used for the insertion of a second eGFP sequence into the LeGO-iG2 vector to produce the lentivector L/eGFP2 with a more efficient eGFP expression.

All produced constructs were subjected to confirmatory PCR and restriction enzyme analysis in order to verify the sequence identity and integrity. The pMD2.G plasmid expressing the glycoprotein $G$ of the vesicular stomatitis virus (VSV-G) envelope and the second generation lentiviral packaging plasmids psPAX2 and pCMVAR8.91 were purchased from Addgene.

All lentivectors were expanded by transforming Stbl3 Escherichia coli strain (Invitrogen) and purified according to the manufacturer's recommendations for free endotoxin columns (Qiagen).

\subsection{Cationic lipid-mediated transfection}

HEK293, HeLa, Huh-7 and NIH/3T3 cells were cultured in their respective culture media until reaching $80-90 \%$ confluence. Then the cells were washed with PBS and serum-free medium (OptiMEM - Gibco) was added to culture plates. The cells were transfected using Lipofectamine 2000 (Invitrogen). DNA plasmids/Lipofectamine complexes were prepared according to the manufacturer's instructions and added to the cells. After $4 \mathrm{~h}$ incubation at $37^{\circ} \mathrm{C}$, the media was replaced with the culture medium and cells were grown at $37{ }^{\circ} \mathrm{C}$ in $5 \% \mathrm{CO}_{2}$. 


\subsection{In vitro transcription and mRNA purification}

For mRNA synthesis, the pMACS constructs (pMACS-CIITA, pMACS-eGFP and pMACS-LNGFR) were previously linearized by digestion with BstZ171 enzyme (New England Biolabs) at the LNGFR gene $3^{`}$ end. Alternatively, the linear template from pMACS-CIITA could be produced by PCR amplification of a fragment comprising the $\mathrm{T} 7$ promoter and the CIITA-IRES-LNGFR sequence, using the following primers: (i) Forward - AGCTCTCTGGCTAACTAGAGAACCCACTGC; (ii) Reverse - CCGCATCCCCAGCATGCCTGCTATAATCAG. Approximately $1 \mu \mathrm{g}$ of each linearized plasmid was utilized as a template for in vitro transcription using the mMessage mMachine T7 Ultra kit (Life Technologies), according to manufacturer's instructions. The produced mRNA was purified by precipitation in lithium chloride $(7.5$ $\mathrm{M} \mathrm{LiCl,} 50 \mathrm{mM}$ EDTA) provided by the transcription kit, following the manufacturer's recommendations. Purified mRNA was then resuspended in a TE buffer (10 mM Tris$\mathrm{HCl}, 1 \mathrm{mM}$ EDTA, $\mathrm{pH} 8.0$ ), quantified by spectrophotometry at a wavelength of 260 nm and stored at $-70{ }^{\circ} \mathrm{C}$.

The mRNAs quality and integrity were certified through denaturing RNA electrophoresis in TBE agarose gel. The $0.7 \%$ gel was prepared by melting agarose in TBE buffer ( $89 \mathrm{mM}$ Tris borate, $2 \mathrm{mM}$ EDTA, $\mathrm{pH} 8.0$ ) containing $0.1 \%$ sodium dodecyl sulfate (SDS - BioRad, Hercules, CA). mRNA samples were diluted in nucleic acid sample buffer (BioRad) supplemented with 0.1\% SDS and pre-heated to $70{ }^{\circ} \mathrm{C}$ for 2 minutes, then cooled in ice. Electrophoresis was performed in TBE buffer, followed by staining with ethidium bromide (Fisher Scientific, Pittsburgh, PA). 


\section{9 mRNA electroporation of dendritic cells}

Immature dendritic cells obtained after five days of monocytes differentiation (Section 3.4) were electroporated with the in vitro transcribed mRNAs according to the method previously described ${ }^{155}$. Before electroporation, iDCs were harvested from culture plates, washed three times with IMDM 10\% FBS and once with Opti-MEM medium (Gibco) at $450 \times \mathrm{g}$ for $8 \mathrm{~min}$ at $4{ }^{\circ} \mathrm{C}$ in order to remove any cytokine trace. Next, the cells were resuspended at a final concentration of $3.3 \times 10^{6}$ cells $/ \mathrm{mL}$ in OptiMEM, transferred to a $2 \mathrm{~mm}$ cuvette (200 $\mu \mathrm{L} /$ cuvette) and incubated with mRNA (10 $\mu \mathrm{g} / 10^{6}$ cells) for 10 minutes on ice. iDCs were then electroporated using a BTX ECM 830 Electro Square Porator (Harvard Apparatus, Holliston, MA) with one square wave pulse at 300-600 V, 0.75 milliseconds length. After electroporation, cells from each cuvette were quickly transferred to one well in a 24-well plate containing $1 \mathrm{~mL}$ of IMDM $10 \% \mathrm{FBS}$, and incubated at $37{ }^{\circ} \mathrm{C}$ and $5 \% \mathrm{CO}_{2} .48$ hours after electroporation, cells viability and transfection efficiency were analyzed by flow cytometry. Nontransfected and transfected iDCs were stained with the Live/Dead Viability/Cytotoxicity Kit (Invitrogen) (Section 3.18.4) and evaluated for eGFP expression.

\subsection{Generation of Lentiviral Gene Transduction Vector Particles}

Production of lentiviral vector particles expressing CIITA and eGFP (LVCIITA) or only eGFP (LV-control), driven by the SFFV promoter and pseudotyped with the VSV-G envelope protein, was achieved by co-transfecting $293 \mathrm{~T}$ cells with

L/CIITA or L/eGFP2 together with pMD2.G and psPAX2 as previously described ${ }^{156}$, 
with minor modifications. Briefly, 5 x $10^{6} 293 \mathrm{~T}$ cells were seeded in $10 \mathrm{~cm}$ plates with $10 \mathrm{~mL}$ of medium and incubated at $37{ }^{\circ} \mathrm{C}$ and $5 \% \mathrm{CO}_{2}$. After 18 hours, the cells were co-transfected with $10 \mu \mathrm{g}$ of L/CIITA or L/eGFP2, $6.5 \mu \mathrm{g}$ of psPAX2 and $2 \mu \mathrm{g}$ of pMD2G.VSV-G by the calcium phosphate method using the Calphos Mammalian Transfection Kit (Clontech, Mountain View, CA), in the presence of $25 \mu \mathrm{M}$ chloroquine (Sigma-Aldrich). 8 hours post-transfection, the culture supernatant was replaced with 10 $\mathrm{mL}$ of fresh medium, and viral supernatants were harvested after 24 and $48 \mathrm{~h}$, centrifuged at $1,000 \mathrm{Xg}$ for $5 \mathrm{~min}$ at $4^{\circ} \mathrm{C}$, filtered through $0.45-\mu \mathrm{m}$ low protein binding filters (Millipore, Billerica, MA) and concentrated prior to storage. The supernatants were concentrated by ultracentrifugation for $150 \mathrm{~min}$ at 75,000 X $g$ in a Beckmann SW28 rotor. The viral pellets were resuspended in sterile PBS and viral stocks were stored in aliquots at $-70^{\circ} \mathrm{C}$.

\subsection{Lentiviral vector particles titration}

The titers were determined by transducing $293 \mathrm{~T}$ cells in a limiting dilution assay. 293T cells were transferred to 24 well plates at a concentration of $5 \times 10^{4}$ cells per well, and incubated at $37{ }^{\circ} \mathrm{C} / 5 \% \mathrm{CO}_{2}$ for 5 hours. After cells adhesion to the plate, the culture medium was replaced by $500 \mu \mathrm{L}$ of fresh medium containing of $8 \mu \mathrm{g} / \mathrm{mL}$ polybrene (Sigma-Aldrich) and serial dilutions from 1:200 to $1: 200,000$ of the concentrated lentiviral vectors, while maintaining one well without virus (negative control). The plates were then centrifuged at $1,000 \mathrm{Xg}$ for 1 hour at RT, and incubated for 24 hours, when the medium was replaced again by $1 \mathrm{~mL}$ of fresh medium. After additional 48 hours incubation, cells were harvested and the eGFP expression was 
determined by flow cytometry. Titers were determined based on the percentage of eGFP positive cells and calculated according to the following formula:

Titer $=(\mathrm{G} \times \mathrm{N} \times \mathrm{D} \times 1000) / \mathrm{V}$, where $\mathrm{G}=$ percentage of eGFP+ cells, $\mathrm{N}=$ number of cells at time of transduction $\left(5 \times 10^{4}\right.$ cells $), \mathrm{D}=$ fold dilution of vector used in the transduction, $\mathrm{V}=$ volume $(\mu \mathrm{L})$ of diluted vector sample in each well.

The virus titer was expressed as transducing units $/ \mathrm{mL}(\mathrm{TU} / \mathrm{mL})$. The lentiviral vector preparations displayed titers of $1.5-2 \times 10^{7} \mathrm{TU} / \mathrm{mL}$ in $293 \mathrm{~T}$ cells.

\subsection{Lentiviral Gene Transfer}

HEK293, HeLa and Huh-7 cells were incubated at $5 \times 10^{4}$ cells per $500 \mathrm{~mL}$ of their respective growth media in the presence of $8 \mu \mathrm{g} / \mathrm{mL}$ polybrene and centrifuged at $1,000 \times \mathrm{g}$ for $1 \mathrm{~h}$ at $\mathrm{RT}$ in the presence of lentiviral vector preparations at a multiplicity of infection (MOI) of 2 , followed by incubation at $37^{\circ} \mathrm{C}$ in $5 \% \mathrm{CO}_{2}$. The medium was changed $24 \mathrm{~h}$ after transduction; cells were cultured for 2 additional days, and analyzed by flow cytometry.

Monocytes purified after $2 \mathrm{~h}$ of plastic adherence (as described in Section 3.4) were exposed to lentiviral vector preparations with a $\mathrm{MOI}$ of 2 , in a $500 \mu \mathrm{L}$ volume in the presence of $4 \mu \mathrm{g} / \mathrm{mL}$ polybrene and centrifuged at $485 \mathrm{xg}$ for $1 \mathrm{~h}$ at $\mathrm{RT}$, followed by incubation at $37{ }^{\circ} \mathrm{C}$ in $5 \% \mathrm{CO}_{2} .24 \mathrm{~h}$ post-transduction, medium was replaced by fresh medium containing 1,000 IU/mL rhu GM-CSF (Sanofi-Aventis) and IL-4 (R\&D Systems), followed by a 7 day culture for dendritic cells differentiation. The immature 
dendritic cells were then harvested for analysis by flow cytometry and quantitative realtime PCR (qRT-PCR).

\subsection{Inoculation of healthy human skin explant with lentiviral vectors}

Human healthy skin explants discarded from plastic surgery (Plastic Surgery Department, University of Pittsburgh Medical Center, Pittsburgh, USA) were inoculated with $100 \mu \mathrm{L}$ of lentiviral vector preparations at a titer of $2 \times 10^{7} \mathrm{TU} / \mathrm{mL}$ using a stabbing method by bifurcated needles. Skin explants stabbed with bifurcated needles without lentivirus were used as mock controls. The inoculated skin was incubated at 37 ${ }^{\circ} \mathrm{C}$ for $2 \mathrm{~h}$. After incubation, the skin surface was washed with PBS and wiped with sterile gauze pads before returning to incubator. $48 \mathrm{~h}$ post-inoculation, the skin was sucrose infused (30\% sucrose for $18 \mathrm{~h}$ at $4^{\circ} \mathrm{C}$ - Sigma-Aldrich), snap-frozen and sectioned to 6-7 $\mu \mathrm{m}$ thickness on a Cryotome (Center for Biologic Imaging, University of Pittsburgh) for immunohistochemistry. Sections were thawed for 5 minutes at RT, rehydrated with PBS, and blocked with 5\% goat serum (Thermo Scientific) for $18 \mathrm{~h}$. The anti-CIITA mouse antibody (E-12, Santa Cruz Biotechnology, Dallas, TX) diluted 1:50 in $0.5 \%$ goat serum was incubated with sections for $1 \mathrm{~h}$ at RT. The Alexa Fluor 549 goat anti-mouse IgG1 (Invitrogen) diluted 1:250 in 0.5\% goat serum was incubated with sections for 45 min at RT followed by a DNA staining step using Hoechst (Thermo Scientific). Cover slips were attached with Gelvatol (Millipore). The Olympus Fluoroview 1000 confocal microscope (Olympus America, Center Valley, PA) was used to view the fluorescent staining and capture microscope images. Images were 
collected using Nikon NIS Element 4.0 imaging software running on a Window 7 64-bit PC (Center for Biologic Imaging, University of Pittsburgh).

\subsection{Immunofluorescence and Confocal Microscopy}

Cells grown on 12-mm-diam poly-D-lysine/laminin coated coverslips (Thermo Scientific) in 24-well plates were transfected with p/CIITA. 24 h post-transfection, cells were rinsed in Dulbecco`s Phosphate-Buffered Saline (DPBS - Mediatech, Manassas, VA), fixed with $1 \%$ formaldehyde in DPBS for $30 \mathrm{~min}$ at RT and permeabilized with cold methanol for $10 \mathrm{~min}$ at $-20^{\circ} \mathrm{C}$. After rinsing with DPBS, cells were incubated at RT for $30 \mathrm{~min}$ in a blocking solution (BS) containing 3\% bovine serum albumin (BSA; Sigma-Aldrich), $100 \mathrm{mM}$ glycine, $10 \%$ fetal bovine serum and $0.05 \%$ Tween-20 in DPBS. The anti-CIITA mouse antibody (E-12, Santa Cruz Biotechnology) diluted 1:50 in BS was incubated with cells for $1 \mathrm{~h}$ at RT. After washing steps with PBS-T (PBS 0.1\% Tween-20), the Alexa Fluor 488 goat anti-mouse IgG1 (Invitrogen) diluted 1:200 in BS was incubated with cells for 45 min at RT followed by a DNA staining step using DAPI (1:5,000 dilution - Invitrogen) for two min at RT and mounting with an antifade mounting medium (ProLong, Molecular Probes, Eugene, OR). The study of CIITA expression in fixed cells was performed using a laser-scanning confocal microscope (Olympus Fluoview 1000). Images were collected using Nikon NIS Element 4.0 imaging software running on a Window 7 64-bit PC. Fixed cells were also analyzed using the fluorescence microscope (Zeiss Axio Imager M1 - Zeiss, Jena, Germany) and digital images were captured using the AxioVision software (Center for Vaccine Research, University of Pittsburgh). 


\subsection{Cell lysates preparation and total protein quantification}

Cell lines were seeded in 6-well tissue culture plates and transfected with CIITA-encoding plasmids after $24 \mathrm{~h}$. Twelve to $96 \mathrm{~h}$ post-transfection, the cells were washed in PBS, centrifuged at $450 \mathrm{Xg}$ for $10 \mathrm{~min}$ at $4{ }^{\circ} \mathrm{C}$ and lysed with $80 \mu \mathrm{L} /$ well of chilled radioimmunoprecipitation assay (RIPA) buffer (10 mM Tris- $\mathrm{HCl}(\mathrm{pH} 7.4), 5$ mM EDTA, $150 \mathrm{mM} \mathrm{NaCl}, 1 \%$ Triton X-100, 1\% deoxycholate, $0.1 \%$ SDS, $1 \%$ aprotinin, $0.1 \mathrm{mM} \mathrm{Na} \mathrm{VO}_{4}-$ Sigma-Aldrich) supplemented with 1x protease inhibitor mixture (Roche Applied Science, Indianopolis, IN) and $100 \mu \mathrm{g} / \mathrm{mL}$ phenylmethanesulfonyl fluoride (PMSF - Sigma-Aldrich), followed by a $10 \mathrm{~min}$ incubation on ice. Monocytes and DCs were washed in PBS, centrifuged at $450 \mathrm{Xg}$ for $10 \mathrm{~min}$ at $4^{\circ} \mathrm{C}$ and resuspended in chilled supplemented RIPA buffer $\left(50 \mu \mathrm{L}\right.$ per $10^{6}$ cells), followed by $10 \mathrm{~min}$ incubation on ice.

Cell lysates were centrifuged for $10 \mathrm{~min}$ at $10,600 \mathrm{Xg}$ at $4^{\circ} \mathrm{C}$ and the supernatants were transferred to new tubes and kept on ice while performing the total protein quantification by bicinchoninic acid (BCA) protein assay (Thermo Scientific) per manufacturer`s instructions. Cell lysates were then normalized for total protein concentration, diluted 1:2 with sample buffer (Amresco, Solon, $\mathrm{OH}$ ) supplemented with $\beta$-mercaptoethanol $(50 \mu \mathrm{L} / \mathrm{mL})$ and boiled at $95{ }^{\circ} \mathrm{C}$ for $5 \mathrm{~min}$. The denatured lysates were then incubated on ice for two min and stored at $-20{ }^{\circ} \mathrm{C}$. 


\subsection{Western blotting}

Approximately $15-100 \mu \mathrm{g}$ total proteins from cell lysates were separated by $8 \%$ SDS-PAGE gel electrophoresis. Gels were transferred to polyvinylidene fluoride (PVDF) membranes (Thermo Scientific), which were blocked for $1 \mathrm{~h}$ at RT in 5\% skimmed milk/PBS-T. For the CIITA protein detection, the membranes were individually incubated overnight at $4{ }^{\circ} \mathrm{C}$ with anti-CIITA mAb (7-1H; Santa Cruz Biotechnology) diluted 1:100 in 5\% skimmed milk/PBS-T. The PML proteins detection was performed after one-hour incubation at RT with anti-PML rabbit antibody (ABD030; Jena Biosciences, Jena, Germany) diluted 1:250. MHC II molecules were detected by incubating membranes for $1 \mathrm{~h}$ at RT with anti-HLA-DR $\alpha$ mAb (FL-254; Santa Cruz Biotechnology) diluted 1:1,000. The $\beta$-actin detection used as loading control was performed by one-hour incubation at RT with anti- $\beta$-actin mAb (BA3R; Thermo Scientific) diluted 1:15,000. After incubation with protein-specific primary antibody, membranes were washed three times for 15 min each with PBS-T and subsequently incubated for $1 \mathrm{~h}$ at $\mathrm{RT}$ with horseradish peroxidase-conjugated species-specific secondary antibodies (Millipore) diluted 1:30,000. After three additional 15 min washes with PBS-T, signal was detected using ECL reagent (Amersham Pharmacia, Piscataway, NJ) on imaging film.

\subsection{Proteasome Activity Assay}

HEK293, HeLa and Huh-7 cells were plated in 6-well tissue culture plates and transfected with p/CIITA after 24 h. 24 h post-transfection, cells were treated with 40 
$\mu \mathrm{g} / \mathrm{mL}$ cycloheximide (Thermo Scientific) and/or $20 \mu \mathrm{M}$ MG-132 (Millipore). In order to analyze the CIITA protein degradation, cells were treated with cycloheximide for 0$16 \mathrm{~h}$, and cell lysates were produced in $4 \mathrm{~h}$ intervals as mentioned at Section 3.8. For the proteasome activity assay, cells were treated with cycloheximide and/or MG-132 for 0$8 \mathrm{~h}$, and cell lysates were produced at the end of treatments. BCA protein assay was used to normalize for total protein concentration and the CIITA protein detection was performed using western blot assays as described in Section 3.9. Equal loading was determined in lysates by $\beta$-actin detection.

\subsection{Flow Cytometry}

\subsubsection{Surface staining of human cell lines}

For the detection of surface MHC class II and costimulatory molecules expressed by cells transfected with p/CIITA, the human cell lines were stained with monoclonal antibodies purchased from BD Biosciences (San Jose, CA) targeting the following molecules: HLA-DR,-DP,-DQ (MHC II molecules), CD40 (costimulatory molecule), CD80 and CD86 (B7-1 and B7-2, costimulatory molecules). The antibodies were used at various dilutions determined by a previous titration in PBMCs. The following fluorescently labeled antibodies were used: fluorescein isothioyanate (FITC) anti-HLA-DR,-DP,-DQ (Tu39), Alexa Fluor 700 anti-CD40 (5C3), phycoerythrin (PE) anti-CD80 (L307.4), FITC anti-CD86 (FUN-1) and their respective isotype controls.

After cells counting with Neubauer Chamber, approximately $10^{6}$ cells were washed twice with PBS at $450 \mathrm{X} g$ for $5 \mathrm{~min}$ at $4{ }^{\circ} \mathrm{C}$, transferred to 96 -well round bottom plates and incubated with LIVE/DEAD Fixable Blue Dead Cell Stain kit 
(Invitrogen), diluted 1:1,000 in PBS, for $20 \mathrm{~min}$ on ice and protected from light. Cells were then washed twice with FACS buffer (1 M HEPES, 5\% BSA, 0.1 M EDTA, 1x PBS, pH 7.4) at $860 \mathrm{Xg}$ for $5 \mathrm{~min}$ at $4{ }^{\circ} \mathrm{C}$ and incubated for $20 \mathrm{~min}$ at $4{ }^{\circ} \mathrm{C}$ with the fluorescently labeled antibodies diluted in FACS buffer. After two additional washes with FACS buffer, cells were fixed in FACS buffer containing 1\% formaldehyde, transferred to polypropylene tubes and analyzed by flow cytometry using an LSR II (BD Biosciences) and the FlowJo 887 software (FlowJo, LLC, Ashland, OR).

\subsubsection{Surface and intracellular detection of MHC II molecules in human cell lines}

For the detection of surface MHC class II molecules together with the recently synthesized MHC II molecules that were not transported to the surface of cells transduced with LV-CIITA, HEK293, HeLa and Huh-7 cells were subjected to surface and intracellular staining with a monoclonal antibody, which targeted the HLA-DR molecule. The fluorescently labeled antibody used was allophycocyanin-Cy7 (APCCy7) anti-HLA-DR (L243 - BD Biosciences). NIH/3T3 cells transfected with p/CIITA were subjected to a similar staining to detect mice MHC II molecules (I-A/I-E) using the antibody PE anti-mouse MHC II (M5/114.15.2, Anfimetrix, Santa Clara, CA).

After LIVE/DEAD and MHC II staining performed as described in the previous Section (3.18.1), cells were fixed and permeabilized using a BD Cytofix/Cytoperm Fixation/Permeabilization Solution Kit (BD Biosciences) according to manufacturer`s instructions. Subsequently, cells were washed once with PermWash buffer at $860 \mathrm{X} g$ for 5 min at $4{ }^{\circ} \mathrm{C}$ and incubated for $45 \mathrm{~min}$ at RT (protected from light) with the same fluorescently labeled anti-HLA-DR mAb diluted in PermWash buffer. Cells were then 
washed once with PermWash buffer and then once with FACS buffer at $860 \mathrm{X} g$ for 5 min at $4{ }^{\circ} \mathrm{C}$, and finally resuspended in FACS buffer containing $1 \%$ formaldehyde for flow cytometry analysis.

\subsubsection{Gating strategy for human cell lines analysis}

In order to analyze the surface and intracellular molecules expressed by CIITAtransfected cell lines, the population of cells presenting the proper size (FSC - forward scatter) and granularity (SSC - side scatter) was selected. Thereafter, doublets were excluded and the molecules of interest were evaluated within the viable cells population. Examples of gating strategies for each human cell line are demonstrated in Figure 3. 
A

HEK293
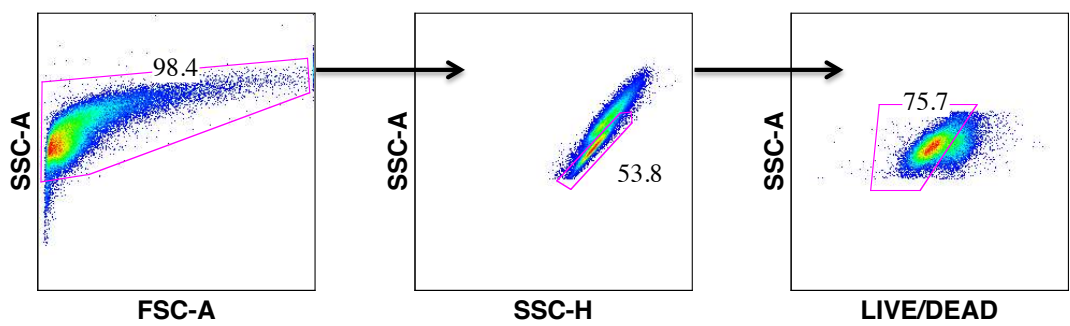

LIVE/DEAD

B

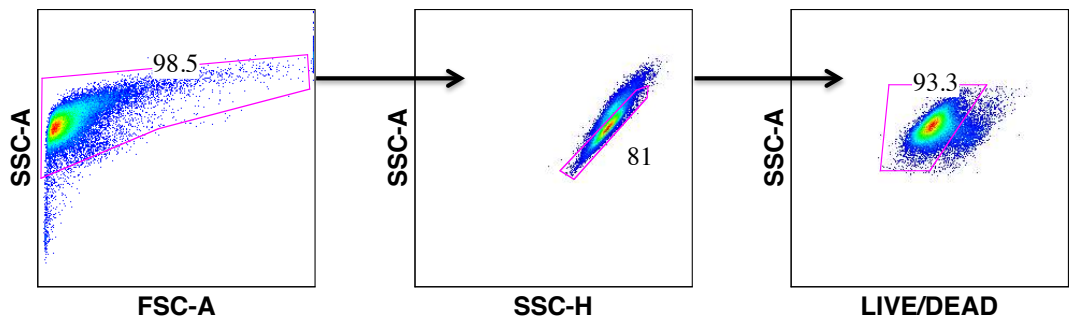

$\mathrm{C}$

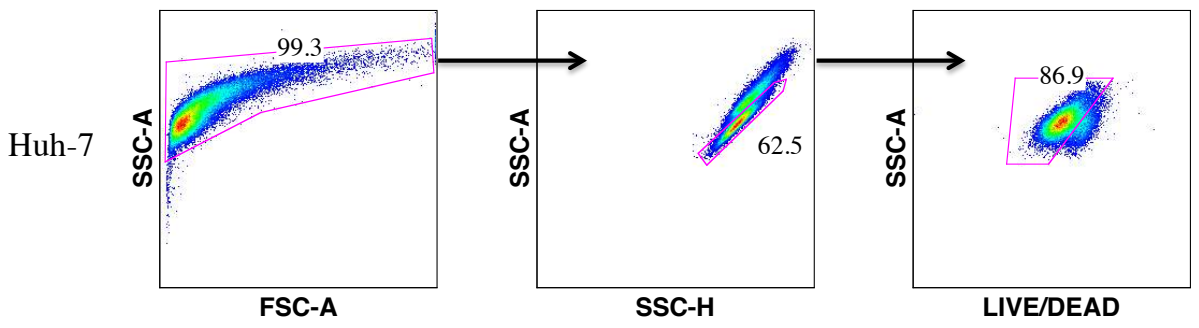

Figure 3 - Gating strategies for the analysis of human cell lines expressing CIITA. HEK293 (A), HeLa (B) and Huh-7 cells (C) were collected after transfection with p/CIITA or transduction with LV-CIITA and analyzed by flow cytometry for the expression of MHC II and costimulatory molecules. Figure representing the gating strategies used for each cell line analysis. Left: dot plots showing the gate delineating cells with proper size (FSC-linear) and granularity (SSC-log). Center: dot plots representing the exclusion of doublets by gating the region in SSA-A and SSA-H presenting single cells. Right: dot plots representing the exclusion of dead cells by gating the live cells unstained with LIVE/DEAD, in which the molecules of interest analysis were performed.

\subsubsection{Surface staining of dendritic cells}

For the phenotypic analysis of dendritic cells, the iDCs and mDCs were stained with monoclonal antibodies purchased from BD Biosciences targeting the following molecules: CD14 (monocytes/macrophages marker), CD11c (myeloid dendritic cells marker), HLA-DR (MHC II molecule), CD40 (costimulatory molecule), CD80 (B7-1, costimulatory molecule) and CD83 (mature dendritic cells marker). The antibodies were used at various dilutions determined by previous titration in PBMCs. The following fluorescently labeled antibodies were used: Pacific Blue anti-CD14 (M5E2), 
phycoerythrin with CF954 (PE-CF954) anti-CD11c (B-ly6), APC-Cy7 anti-HLA-DR (L243), Alexa Fluor 700 anti-CD40 (5C3), PE anti-CD80 (L307.4), allophycocyanin anti-CD83 (HB15e), and their respective isotype controls.

After cells counting with Neubauer Chamber, approximately 1 to $2 \times 10^{5}$ cells were stained and analyzed by flow cytometry as previously describe in Section 3.18.1.

\subsubsection{Surface and intracellular detection of MHC II molecules in dendritic cells}

For the detection of surface MHC class II molecules together with the recently synthesized MHC II molecules that were not transported to the surface of iDCs after transduction with LV-CIITA, the cells were subjected to surface staining as described in the previous section (3.18.4), followed by intracellular staining with the HLA-DR-APC$\mathrm{Cy} 7 \mathrm{mAb}$. The intracellular staining was performed as described in Section 3.18.2.

\subsubsection{Gating strategy for dendritic cells analysis}

In order to analyze the phenotype of dendritic cells, the population of cells presenting the proper size and granularity was selected, followed by the exclusion of doublets and dead cells. The molecules of interest were evaluated in the population of CD14 CD11 ${ }^{+}$cells, excluding macrophages and monocytes from analysis. Examples of gating strategies for dendritic cells derived from healthy and HIV-infected individuals are demonstrated in Figure 4. 
A
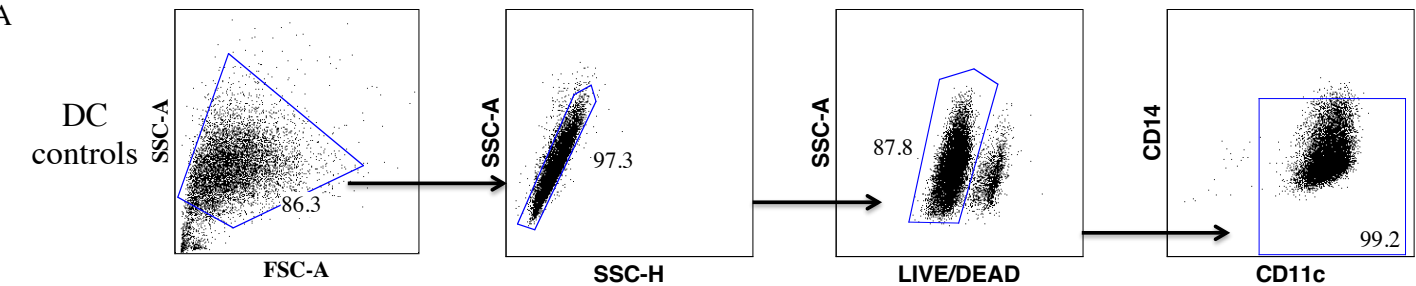

B

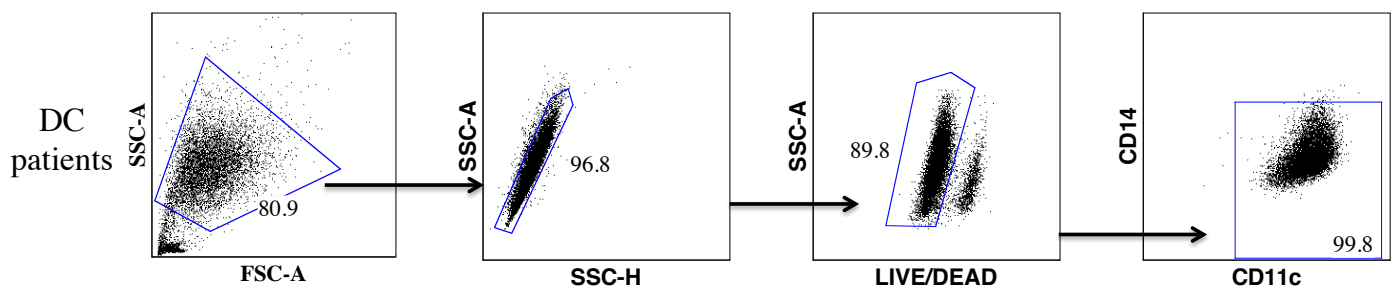

Figure 4 - Gating strategies for dendritic cells phenotypic analysis. iDCs and the subtypes of mDCs obtained from healthy donors (A) and HIV-infected individuals (B) were collected after seven days of differentiation culture and analyzed according to the surface molecules and MHC II expression through flow cytometry. Figure representing the gating strategies used for iDCs and mDCs. Left: dot plots showing the gate delineating cells with proper size (FSC-linear) and granularity (SSC-linear). Center: dot plots representing the exclusion of doublets followed by dot plots representing the exclusion of dead cells. Right: dot plots representing CD14 and $\mathrm{CD} 11 \mathrm{c}$ where the $\mathrm{CD} 14 \mathrm{CD}^{-} 1 \mathrm{c}^{+}$cells were gated, selecting the population of interest for phenotypic analysis.

\subsection{Dendritic cells activation and Interleukin-12(p70) production}

The immature dendritic cells and the differentially polarized mature dendritic cells obtained after seven days of culture (Section 3.4) were harvested and washed with PBS, counted and transferred to $96-$ well plates at a concentration of $2.5 \times 10^{4}$ cells/well in $100 \mu \mathrm{L}$ of IMDM medium containing $1 \%$ FBS. To mimic the activation stimulus provided by CD4+ T helper cells expressing CD40L, J558 cells stably transfected with CD40L (kindly provided by Dr. P. Lane - University of Birmingham/England) were added to the wells at a concentration of $5 \times 10^{4}$ cells $/ 100 \mu \mathrm{L}$ of medium. After 48 hours of co-culture at $37{ }^{\circ} \mathrm{C}$ and $5 \% \mathrm{CO}_{2}$, the culture supernatants were collected for IL12(p70) dosage by enzyme-linked immunosorbent assay (ELISA). Unstimulated cells were used as a negative control. 
The ELISA assay was performed according to the protocol established by Dr. Robbie Mailliard (University of Pittsburgh/USA). The 96-well EIA/RIA plate (SigmaAldrich) was sensitized for $18 \mathrm{~h}$ at RT with $4 \mu \mathrm{g} / \mathrm{mL}$ of anti-IL-12(p70) mAb (clone 20C2 - Thermo Scientific) diluted in PBS. The solution was removed and the plate blocked with PBS containing 2\% BSA for $1 \mathrm{~h}$ at RT. After this step, the plate was washed three times in wash buffer (50 mM Tris- $\mathrm{HCl}, 0.4 \%$ Tween) and incubated for 1 $\mathrm{h}$ at RT with a standard curve of rhu IL-12(p70) protein (BD Bioscience) diluted in IMDM medium $(5,000-9.7 \mathrm{ng} / \mathrm{mL})$ and with $50 \mu \mathrm{L}$ of DCs culture supernatants. After removal of the solutions, the biotinylated mAb anti-IL-12(p70) $(0.54 \mu \mathrm{g} / \mathrm{mL}-$ Thermo Scientific) was incubated for $1 \mathrm{~h}$ at RT, followed by three washes with wash buffer. Then, peroxidase streptavidin (Thermo Scientific) was added to the microplate at 1:7,500 and incubated at RT for $30 \mathrm{~min}$. The enzymatic activity was detected after incubation for 15 min at RT with tetramethylbenzidine (TMB - Thermo Scientific) and neutralization by adding $1 \mathrm{M}$ sulfuric acid. The immunoenzymatic reaction optical density was determined in a spectrophotometer at $450 \mathrm{~nm}$ (XS PowerWave - Biotek, Winooski, VT). The cytokine concentration was calculated through linear regression analysis based on the standard curve, using GraphPad Prism 5 software (La Jolla, CA).

\subsection{RNA Isolation and Quantitative Real-Time PCR}

Quantitative real-time PCR (qRT-PCR) was performed as described elsewhere ${ }^{157,158}$. Briefly, total RNA was extracted from whole cells using the PicoPure ${ }^{\circledR}$ RNA Isolation Kit (Life Technologies) according to manufacturer`s protocol. cDNA

synthesis was performed using SuperScriptTM III Reverse Transcriptase (Life 
Technologies), as per manufacturer's guidelines. Primers sequences were designed based on the synthetic CIITA sequence mentioned in Section 3.5, as follows: forward, 5'-CCAGCCAGCGGACAAATGAG-3'; reverse, 5'CTCGCCGTGGTAGATGAATATGCT-3'. The primers were able to detect both synthetic and endogenous CIITA mRNAs. PCR reaction mix contained $132 \mathrm{ng}$ of cDNA, $10 \mu \mathrm{L}$ of Platinum SYBR qPCR SuperMix-UDG (Life Technologies), $1 \mu \mathrm{L}$ of each primer at an initial concentration of $50 \mu \mathrm{M}$ and sterile water up to the final volume of $20 \mu \mathrm{L}$. Samples were applied into MicroAmp® Optical 96-well Reaction Plate (Life Technologies), in triplicate, and ran in a 7900HT Fast Real-Time PCR System (Life Technologies) using its setup program with minor modifications (volume to $20 \mu \mathrm{L}$ and number of cycles to 60). Transcription levels were calculated with the $2 \Delta \Delta \mathrm{Ct}$ $\operatorname{method}^{159}$. For the normalization of the qPCR values, $\beta$-actin was used as a housekeeping gene (forward, 5'- GGCACCACACCTTCTACAATGAG-3', and reverse, 5' - CGTCATACTCCTGCTTGCTGATC-3').

\subsection{Statistical analysis}

Values were compared using a two-tailed paired Student $t$ test when there were only two groups or two-way ANOVA followed by the Bonferroni post-test for multiple comparisons. Analyses were performed using GraphPad Prism 5 software, and differences were considered significant at $\mathrm{p}<0.05$. 
4 RESULTS 


\subsection{CIITA sequence design and optimization for expression in human cells}

The human CIITA expression is tightly regulated at the transcriptional ${ }^{160-165}$ and post-transcriptional levels ${ }^{35,166}$ in a cell type-specific manner. In order to avoid cisacting elements present in the 3' UTR of CIITA mRNA that mediate the transcript degradation $^{71,72}$ and guarantee protein expression regardless of cell-specific regulatory mechanisms, the CIITA cDNA lacking the $5^{\prime}$ and $3^{\prime}$ UTRs was obtained through reverse translation of the human CIITA amino acid sequence (Figure 5).

This DNA sequence was modified in silico by the fusion with the reverse translated FMDV 2A cDNA (Figure 5), a strategy used to simplify the future construction of bicistronic expression vectors. The insertion of an antigen-of-interest open reading frame $(\mathrm{ORF})$ after the $2 \mathrm{~A}$ sequence would allow the CIITA and antigen co-expression as a single transcript translated into separate proteins, since the $2 \mathrm{~A}$ oligopeptide mediates a ribosome skipping event ${ }^{153,167}$. Thus, the bicistronic constructs would promote the expression of two proteins with equimolar amounts under the control of a single promoter.

The designed sequence CIITA_2A was further modified by the insertion of a Kozak sequence at the 5 '-end to improve translation, and two stop codons at the 3 '-end to prevent erroneous insertions of amino acids into the translated protein. In addition, 5, and 3' extensions encoding cleavages sites were added to facilitate future cloning strategies. The final DNA sequence was then artificially optimized for improved expression in human cells (Figure 5). 


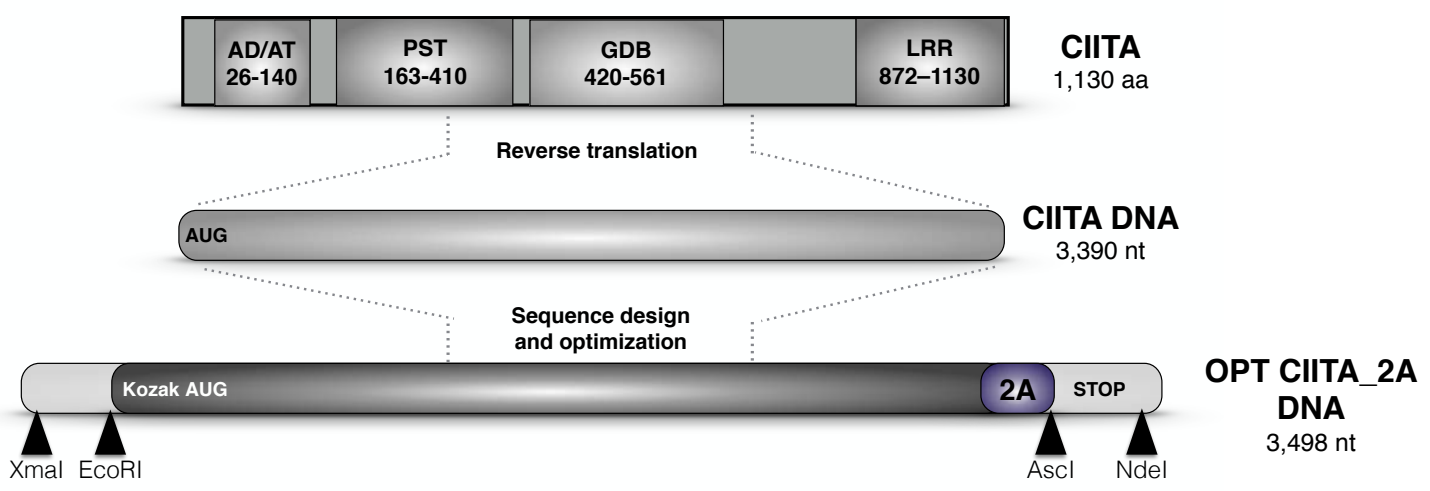

Figure 5 - CIITA sequence design. The 1,130 amino acids (aa) sequence corresponding to the entire CIITA protein, including its functional domains, was used as a template for reverse translation to obtain the CIITA DNA (3,390 nucleotides - nt). This sequence was further modified in silico by the addition of cleavage sites, a Kozak sequence, stop codons and the FMDV 2A sequence, followed by genetic optimization to obtain the final OPT CIITA_2A DNA $(3,498 \mathrm{nt})$. This sequence design method was developed in order to facilitate cloning strategies and guarantee high CIITA expression levels in different human cell types.

The sequence optimization was performed in collaboration with Dr. Rafael Dhalia (Virology and Experimental Virology Laboratory, Aggeu Magalhães Research Center, FIOCRUZ-PE, Brazil) utilizing the genetic algorithm of LETO 1.0 software. Parameters such as codon usage, RNA secondary structure, GC content distribution, repetitive DNA motifs and cryptic splice sites among others, were modified and/or deleted from the original sequence (CIITA_2A) by silent point mutations. The extensions containing cleavage sites were blocked prior to optimization to preserve the desirable sites for cloning strategies. The complete report of the optimization is attached to this thesis (Appendix A), but some important steps in this process are worthwhile to highlight.

First, the genetic algorithm evidenced a significant difference between the frequency of codons presented in the CIITA_2A sequence and the codons preferentially used by human cells for protein expression. Figure 6A exemplifies the optimization of the codon usage parameter by showing the alterations performed with the codons encoding the amino acid Lysine (the same procedure was performed for all amino 
acids). Between the two possible codons assigned to Lysine (AAA and AAG), 2A_CIITA predominantly contains the AAA codon ("Original" - 61.4\%). Note that in human cells ("Target"), the two codons are used with similar frequencies (43 to 57\%). Thus, the codons frequencies present in the original sequence to express Lysine were converted in the optimized sequence ("Optimized") into frequencies similar to those used in human cells. The maintenance of discrepant codon usage in the sequence-ofinterest can impair the translational process in human cells, resulting in the expression of truncated CIITA proteins. Hence, the blue bar in the graph (Figure 6A) represents the discrepancy between codons present in the CIITA_2A sequence to express Lysine and the possible codons used to express the same amino acid in human cells. The longer the bar, the greater the discrepancy between codon usages. The optimization process provides a better codons distribution in order to facilitate expression in human cells. Thus, the bar is significantly reduced in the optimized sequence (green bar - in this example the green bar cannot be visualized since there was around $100 \%$ reduction in the discrepancy).

Second, although the size and the number of mRNA secondary structures are not directly related to low translation efficiency, these structures generally can adversely affect the translation process by hindering the ribosome movement along the mRNA and obstructing the tRNAs access to their respective codons. Therefore, the reduction in the number and length of predicted hairpin-like structures through the optimization process may also favor the protein synthesis efficiency (Figure 6C-D). Lastly, the original sequence was evaluated for the GC content distribution aimed at obtaining more stable structures and consequently improving protein expression (Figure 6B). After the optimization process, a commercial supplier synthesized the in silico-designed CIITA_2A sequence. 
A

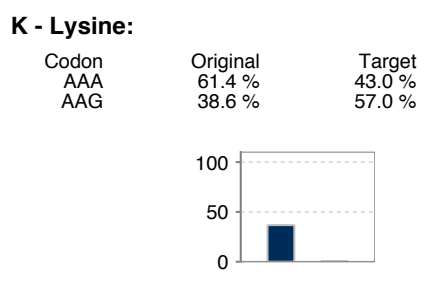

B

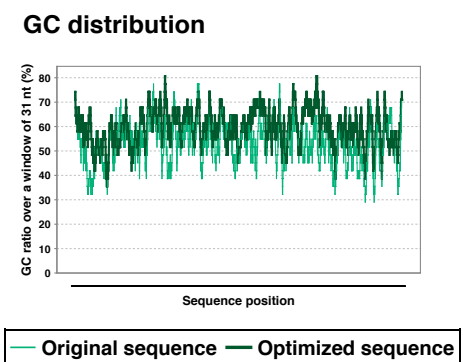

C Original sequence

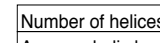

\begin{tabular}{|l|l|}
88 \\
\hline
\end{tabular}

Average helix length

Maximum helix length

Average helix score

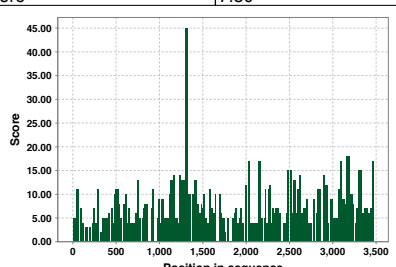

$\mathrm{D}$

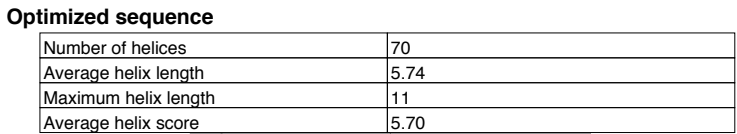

\begin{tabular}{|l|l|l}
\hline Maximum helix length \\
\hline Average helix score \\
\hline
\end{tabular}

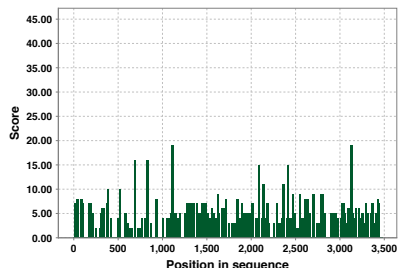

Figure 6 - Optimization of CIITA_2A sequence for improved expression in human cells. (A) Codon usage optimization illustrated by the alterations performed in the codons assigned to the amino acid Lysine. Top: Comparison of the codon frequency of the native CIITA_2A sequence (Original) versus the final optimized CIITA_2A sequence (Optimized). As a reference, the codon usage of the human cells (Target) is included. Bottom: Chart indicating the sum of the deviation from the Target codon usage, for the Original (blue bar) and Optimized (green bar) sequences. Lower bars indicate a better match with the Target codon usage (the absence of the green bar indicates a perfect match). (B) GC distribution optimization. Chart representing the average GC content over a window of $31 \mathrm{nt}$ along the Original and Optimized sequences. (C-D) mRNA secondary structure optimization. Tables and charts indicating the number and length of predicted mRNA secondary structures along the Original (C) and Optimized (D) sequences.

\section{$4.2 \mathrm{p} / \mathrm{CIITA}$ expression vector construction}

Although we produced a synthetic DNA sequence designed for the construction of bicistronic vectors to be applied in future immunotherapeutic strategies, the goal of the present study was the development of gene delivery systems carrying only the designed CIITA sequence. Such systems would allow the validation of protein expression, nuclear import and MHC II transactivation activity in different human cell types as the first step in the evaluation of CIITA as a molecular adjuvant. Therefore, we 
initially produced the construct p/CIITA by inserting the optimized sequence lacking the $2 \mathrm{~A}$ fusion into the p43.2 vector (Figure 7), a versatile CMV expression vector suitable for DNA vaccine formulations ${ }^{168}$ and packaging of adeno-associated virus (AAV) transduction particles ${ }^{169}$. The vector $\mathrm{p} / \mathrm{CIITA}$ was then used for human cell lines transfections.

A Insert amplification strategy

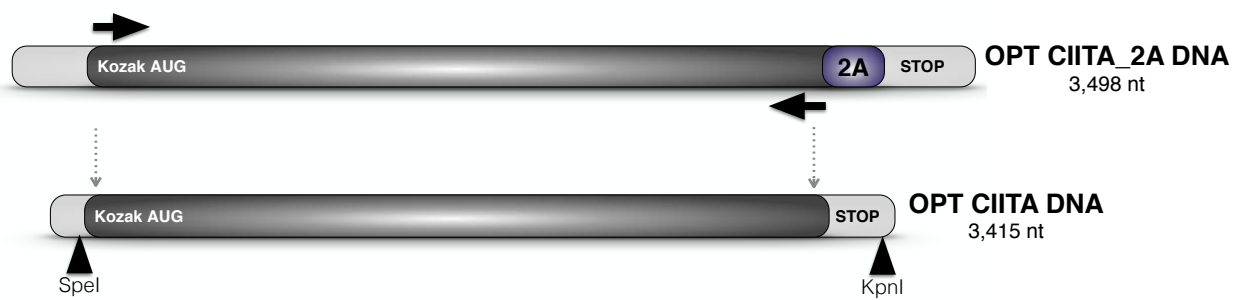

B p/CIITA construct

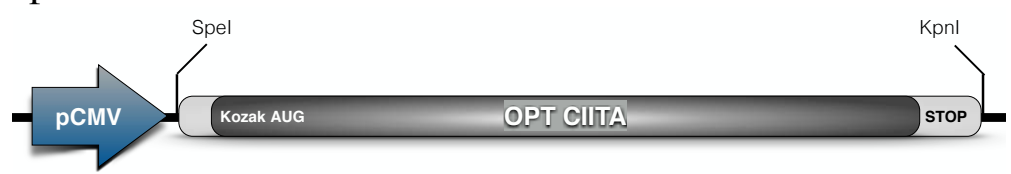

Figure 7 - Strategy for constructing the CMV expression vector p/CIITA. (A) Schematic representation of primers used to amplify the optimized CIITA sequence without $2 \mathrm{~A}$ fusion containing restriction sites necessary for insertion into the $\mathrm{p} 43.2$ vector to generate $\mathrm{p} / \mathrm{CIITA}$. (B) Illustration of p/CIITA construct that presents the inserted sequence driven by a CMV promoter.

\subsection{CIITA protein expression and intracellular distribution in p/CIITA- transfected non-immune cells}

For the developed CIITA construct validation, we investigated whether different non-immune human cell types that do not constitutively express the endogenous CIITA, would efficiently express the transactivation factor after transfection with p/CIITA. Initially, the transfection of HEK293, HeLa and Huh-7 cells was established using an expression vector encoding eGFP in order to achieve approximate transfection efficiencies among the cell lines (Figure 8A). The three cell types were then transfected with p/CIITA and high levels of CIITA mRNA expression were detected $48 \mathrm{~h}$ post- 
transfection (Figure 8B). The quantitative real-time PCR assays were performed in collaboration with Dr. Bruno Douradinha (Center for Vaccine Research, University of Pittsburgh, USA).

A

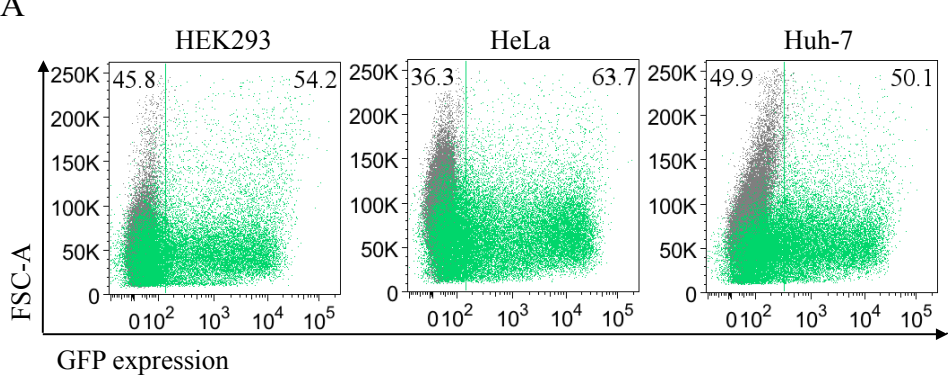

B

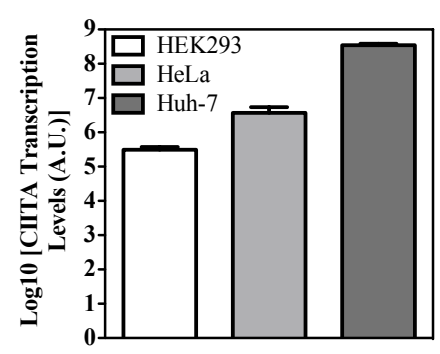

Figure 8 -Transfected HEK293, HeLa and Huh-7 cell lines efficiently express the CIITA mRNA. (A) Plots represent the flow cytometric analysis of eGFP-positive cells frequency in pcDNAl-eGFP-transfected cell lines (green dot-plot) $48 \mathrm{~h}$ post-transfection as a means to determine the transfection efficiencies. Numbers represent the frequency of eGFP-negative cells (left gate) and eGFP-positive cells (right gate). Non-transfected cells were used as negative controls (gray dot-plot). (B) CIITA mRNA levels in p/CIITA-transfected cells. RNA from HEK293, HeLa and Huh-7 cells were analyzed by quantitative (q)RT-PCR for the expression of CIITA $48 \mathrm{~h}$ post-transfection. Relative levels of CIITA mRNA transcription represent arbitrary units normalized to $\beta$-actin RNA transcription. Data are derived from 3 assays and the error bars indicate mean $\pm \mathrm{SD}$.

In order to assess the CIITA protein expression and cellular steady-state localization at $24 \mathrm{~h}$ post-transfection, we used high magnification (40X, 1.3 NA) point scanning confocal microscopy to acquire fluorescent images, in collaboration with the doctoral candidate Parichat Duanghkae (Center for Vaccine Research, University of Pittsburgh, USA). The confocal images showed that all three transfected cell types expressed CIITA uniformly distributed between nucleus and cytoplasm (Figure 9A), consistent with a previously published localization pattern ${ }^{170-172}$. Similar results were obtained with fluorescence microscopy (63X, 1.4 NA - Figure 9B). Figure 9 represents several independent experiments where the protein expression and intracellular distribution were considered invariable in each cell type. Taken together, these results indicate that a variety of non-immune cells can express the CIITA optimized sequence 
and present the protein in the nucleus, a requirement for proper MHC II transactivation activity. 


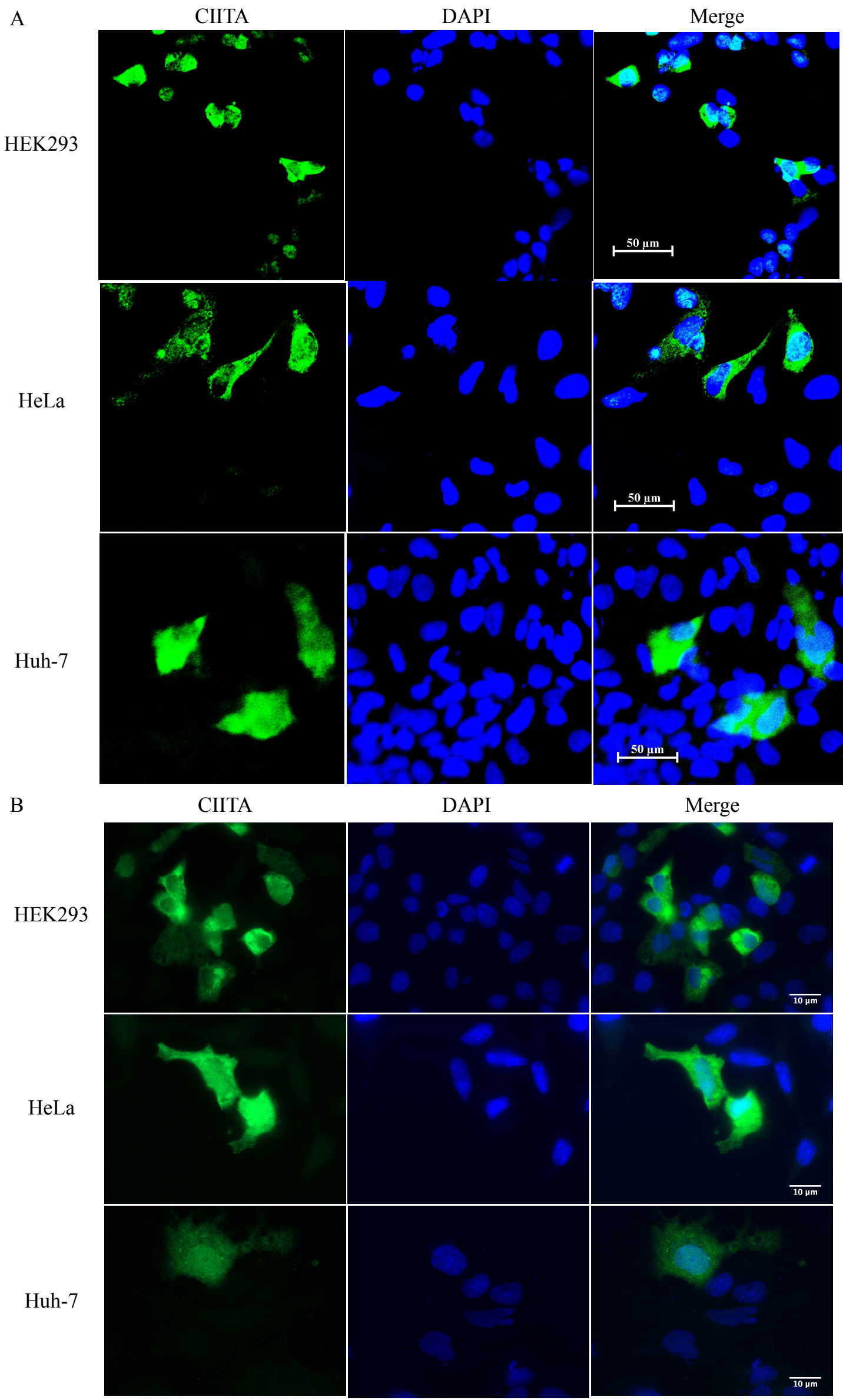


Figure 9 - CIITA is expressed in transfected HEK293, HeLa and Huh-7 cell lines with proper intracellular distribution. Cells were transfected and incubated for $24 \mathrm{~h}$ prior to fixation. Fixed cells were incubated with anti-CIITA ab, followed by incubation with a secondary antibody conjugated with Alexa Fluor-488 dye (green). DAPI (blue) counterstains nuclei. Cells were analyzed by point scanning confocal microscopy (40X, 1.3 NA) (A) and immunofluorescence microscopy (63X, 1.4 NA) (B). Merged images show CIITA presence in all three cell lines nuclei and cytoplasm. Scale bars, $50 \mu \mathrm{m}$ (A) and $10 \mu \mathrm{m}$ (B).

\subsection{CIITA transactivation activity in transfected non-immune cells}

To further evaluate the CIITA construct, experiments were performed to assess the encoded protein functionality in the three different cell types. HEK293, HeLa and Huh-7 cells were transfected with p/CIITA, and samples obtained between 24 and $72 \mathrm{~h}$ post-transfection were analyzed by flow cytometry for surface MHC II molecules expression. All cell types presented a gradual increase in the intensity of MHC II expression (Figure 10B) and in the number of positive cells (Figure 10A), when compared with their respective MHC II-negative non-transfected counterparts. Besides the anti-MHC II monoclonal antibody unspecific binding to HeLa cells that could not be eliminated by testing different clones or antibody dilutions (data not presented), the increase in the MHC II expression was clearly evidenced (Figure 10A and B). Cells transfected with the empty p43.2 vector were also MHC II-negative between 24 and 72 $\mathrm{h}$ post-transfection (data not presented). In addition, the p/CIITA transfection of HEK293 cells specifically induced the expression of MHC II, since the detection of costimulatory molecules CD80, CD86 and CD40 by flow cytometry was similar between $\mathrm{p} / \mathrm{CIITA}$ transfectants and negative controls at $72 \mathrm{~h}$ post-transfection (Figure 11). The same pattern was observed at 96 and $120 \mathrm{~h}$ post-transfection (data not presented). Thus, the CIITA encoded by the p/CIITA construct is transcriptionally active in different non-immune cells and its expression is sufficient for the specific 
induction of target genes such as MHC II, as previously reported for the endogenous CIITA $^{38,99,173}$.

However, the analyzed cell types surprisingly presented striking differences in the kinetics of MHC II expression, even though all three transfected cell lines expressed high levels of CIITA mRNA (Figure 8B). While the transfection of both HEK293 and HeLa cells resulted in the appearance of MHC II molecules after $48 \mathrm{~h}$, reaching approximately $15 \%$ of positive cells $72 \mathrm{~h}$ post-transfection, a maximum of $2 \%$ of MHC II-positive cells were detected only $72 \mathrm{~h}$ after transfection of Huh-7 (Figure 10A). The $48 \mathrm{~h}$ delay in the MHC II synthesis is consistent with the requirement for CIITA translation prior to MHC II induction ${ }^{99}$, but the significantly lower frequency of positive cells in Huh-7 was unexpected due to its high CIITA mRNA level detected 48 h posttransfection (Figure 8B). The dissimilarities between the cell types were more evident when analyzing MHC II expression levels. Even though HEK293 and HeLa cells showed comparable frequencies of MHC II-positive cells (Figure 10A), HEK293 expressed the highest level of MHC II molecules at $72 \mathrm{~h}$ post-transfection, followed by HeLa and Huh-7 cells, in which the MHC II levels were detected at very low levels (Figure 10B). These distinct MHC II expression kinetics were further confirmed by western blot assays using cell lysates obtained between 12 and $96 \mathrm{~h}$ post-transfection with p/CIITA, which also revealed a sustained MHC II expression for at least $96 \mathrm{~h}$ in the three cell lines (Figure 10C). These differences in the MHC II expression suggest that the CIITA protein levels and/or activity are differently regulated by cell typespecific post-translational mechanisms. 
A

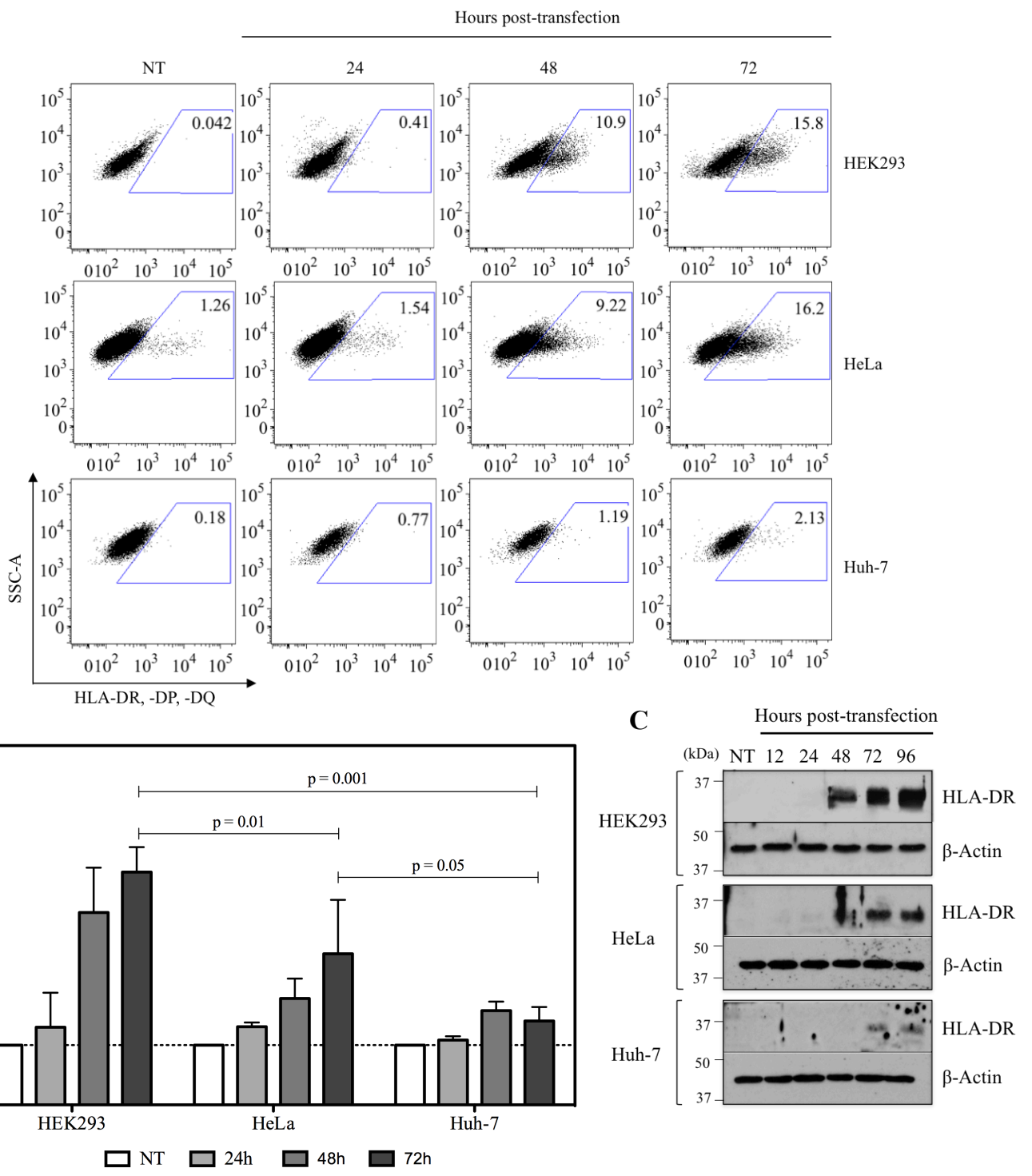

Figure 10 - p/CIITA-transfected cell lines express MHC II molecules at distinct levels and kinetics. (A-B) HEK293, HeLa and Huh-7 cells were transfected with p/CIITA and harvested at 24,48 and $72 \mathrm{~h}$ post-transfection for analysis of HLA-DR/-DP/-DQ surface expression by flow cytometry. The population of interest was defined by exclusion of dead cells and doublets. Nontransfected cells were used to set the gate determining HLA-DR/-DP/-DQ-positive cells. (A) Plots represent the HLA-DR/-DP/-DQ expression in the three cell lines at each interval posttransfection. Numbers in the plots represent the frequency of positive cells. (B) Bar graphs display the fold change in median intensity of fluorescence (MFI) relative to the non-transfected control. Data are means \pm SEM of three independent experiments. $p$ values are indicated. (C) $\mathrm{p} / \mathrm{CIITA}$-transfected cells were harvested at the indicated times, lysed and $15 \mu \mathrm{g}$ of each cell extract were transferred to PVDF membranes. The membranes produced for each cell line were analyzed in parallel by western blot for the HLA-DR expression using the same film exposure time. $\beta$-actin was used as a loading control. Numbers on the left indicate the positions of standard protein marker bands. 

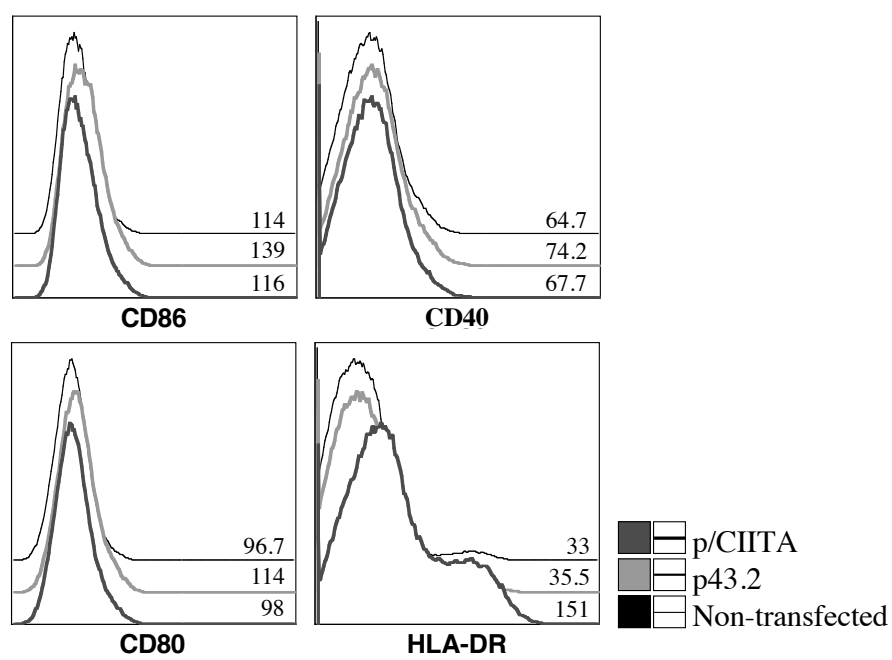

Figure 11 - p/CIITA-transfected HEK293 cells do not express costimulatory molecules. HEK293 cells were transfected either with p/CIITA or with p43.2 and harvested at $72 \mathrm{~h}$ posttransfection for analysis of CD86, CD40 and CD80 surface expression by flow cytometry. HLA-DR surface expression was used as positive control for CIITA activity. The population of interest was defined by exclusion of dead cells and doublets. Non-transfected cells were used to set the gate determining positive cells for each molecule. Histograms show the comparison of molecules MFIs from non-transfected cells, p/CIITA and p43.2 transfectants. Numbers in the histograms represent the absolute MFI of each sample. Results shown are representative of two experiments.

\subsection{Kinetics of CIITA protein expression in transfected non-immune cells}

It is known that the regulation of CIITA intracellular distribution and protein stability are crucial post-translational mechanisms utilized to fine-tune the CIITA transactivation activity ${ }^{39,73,77,80,82,171,174,175}$. Having already demonstrated that the expressed CIITA is present in the nucleus of HEK293, HeLa and Huh-7 cells as early as $24 \mathrm{~h}$ post-transfection (Figure 12), we subsequently investigated whether the CIITA protein levels would vary between 12 and $96 \mathrm{~h}$ post-transfection of the cell lines, using western blot assays. Interestingly, HEK293, HeLa and Huh-7 cells presented varying CIITA expression kinetics (Figure 12). HEK293 cells presented higher and more stable levels of CIITA, which was detected as early as $12 \mathrm{~h}$ and maintained until $96 \mathrm{~h}$ posttransfection. HeLa cells expression was transient, presenting intermediary levels of 
CIITA during the first $24 \mathrm{~h}$ post-transfection, followed by a drastic reduction in protein levels after $48 \mathrm{~h}$ and reaching undetectable levels at $96 \mathrm{~h}$. In contrast, Huh-7 cells presented very low levels of CIITA overall, which was first detected at $12 \mathrm{~h}$, increased to $24 \mathrm{~h}$ and remained stable until $96 \mathrm{~h}$. Importantly, all cells reached the peak of CIITA expression 24 h post-transfection, when HEK293 expressed the highest protein level among the three cell types, followed by HeLa and then Huh-7 cells (Figure 12), conforming with the previously demonstrated MHC II expression pattern (Figure 10). Again, the patterns of both CIITA and MHC II expression did not correspond with differences between cells in transfection efficiencies or CIITA mRNA synthesis (Figure 8A and $\mathrm{B})$. Hence, these findings link the distinct MHC II expression kinetics to variances in the CIITA levels in different non-immune cells, suggesting that the MHC II transactivation activity may be controlled by a cell type-specific regulation of CIITA degradation.

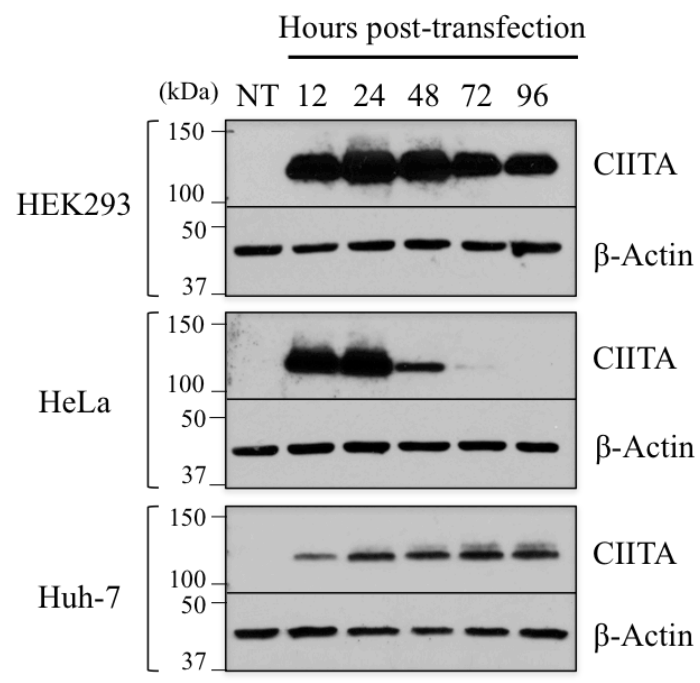

Figure 12 - p/CIITA-transfected cells differ in the level and kinetic of CIITA expression. HEK293, HeLa and Huh-7 cells were transfected with p/CIITA and harvested at the indicated times, lysed and $15 \mu \mathrm{g}$ of each cell extract were transferred to PVDF membranes. The membranes produced for each cell line were analyzed in parallel by western blot for the CIITA expression using the same film exposure time. Non-transfected cells were used as a negative control for the CIITA expression and the detection of $\beta$-actin was used as a loading control. Numbers on the left indicate the positions of standard protein marker bands. Results shown are representative of four experiments. 


\subsection{Influence of proteasomal degradation in the levels of CIITA expression by non- immune cells}

Next we investigated the CIITA degradation mode in p/CIITA-transfected HEK293, HeLa and Huh-7 by treatment with cycloheximide, which blocks the de novo protein synthesis in eukaryotic cells. After cycloheximide treatment, each cell type showed different CIITA degradation patterns (Figure 13). In HEK293, the CIITA protein was slightly reduced and remained stable throughout treatment. In Huh-7 cells, about half of the protein had degraded after $4 \mathrm{~h}$, but the CIITA level was then held stable until 12h. Differently, HeLa cells suffered the highest degradation rate, with a drastic reduction in the CIITA level after $4 \mathrm{~h}$ and reaching almost complete degradation after $12 \mathrm{~h}$ of protein synthesis blocking. No reduction was found in the level of actin used as a control (Figure 13). These results confirm that the three human cell lines degrade the CIITA protein differentially, highlighting the prominent degradation rate in HeLa cells.

Accumulating evidence shows that the CIITA protein is a target for the proteasomal degradation $^{73,77}$, but so far no study has compared the proteasome influence on the mode of CIITA degradation of different cell types. To address this, p/CIITAtransfected cells were subjected to an $8 \mathrm{~h}$ treatment with the proteasome inhibitor MG132 alone or in combination with cycloheximide. The treatment with only MG-132 exclusively blocks the proteasomal degradation while the de novo protein synthesis remains active. In this case, if the proteasome interferes in the basal levels of CIITA, the protein levels in the MG-132-treated cells (Figure 13B, lane 4) must be higher than the levels in the beginning of treatment (Figure 13B, lane 1). In this regard, HEK293 and HeLa cells did not present higher CIITA levels after MG-132 treatment, while Huh-7 
cells did (Figure 13B). These data indicate that the proteasome is constantly degrading newly synthesized CIITA proteins in Huh-7 cells, explaining why these cells presented a lower protein levels (Figure 12) while presenting the highest level of CIITA transcription (Figure 8B).

A

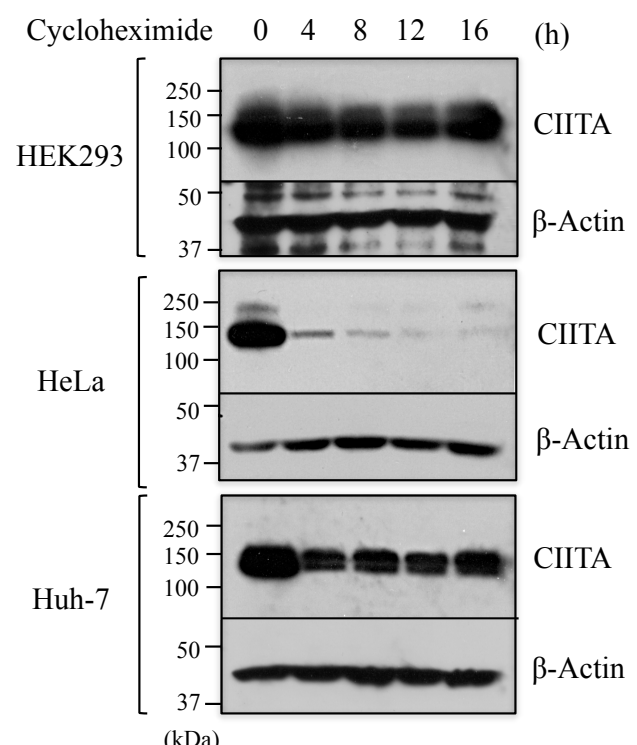

B

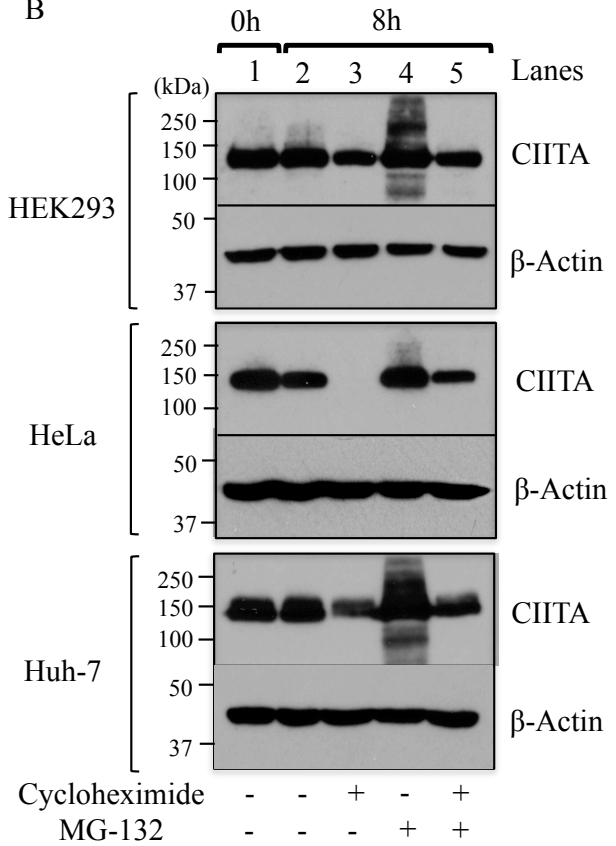

Figure 13 - p/CIITA-transfected cells present distinct CIITA degradation kinetics due to heterogeneous levels of proteasome degradation. (A) CIITA degradation kinetics. HEK293, HeLa and Huh-7 cells were transfected with p/CIITA and incubated for $24 \mathrm{~h}$ to promote CIITA expression. Protein synthesis in these cells was then inhibited by cycloheximide treatment. Samples were removed at the indicated time points, and the amount of CIITA protein was analyzed by western blot. (B) Proteasome influence in the CIITA degradation. p/CIITA transfected cells were incubated for $24 \mathrm{~h}$ to promote CIITA expression. Cells were then treated as indicated with cycloheximide and/or the proteasome inhibitor MG-132 for 8 hours prior to harvesting. The amount of CIITA protein was analyzed by western blot. For the western blot assays (A-B), cells were lysed and cell extracts were transferred to PVDF membranes (30 $\mu \mathrm{g}$ for HEK293 or HeLa extracts and $50 \mu \mathrm{g}$ for Huh-7 extracts). The membranes produced for each cell line were analyzed in parallel by western blot for the CIITA expression using the same film exposure time. $\beta$-actin was used as a loading control. Numbers on the left indicate the positions of the standard protein marker bands. Results shown are representative of three experiments.

The combined treatment with cycloheximide and MG-132 blocks both de novo protein synthesis and proteasomal activity. Thus if the decrease in CIITA level after the cycloheximide treatment (Figure 13B, lane 3) is only a result of proteasomal degradation, the combined treatment with MG-132 (Figure 13B, lane 5) reconstitutes 
the protein to the levels detected in untreated cells (Figure 13B, lane 2). Taking this into account, the different levels of degradation found in HEK293 and Huh-7 cells after cycloheximide treatment were not related to the proteasomal activity and might be a result of another degradation pathways ${ }^{176,177}$ to be further investigated. Since the CIITA expression kinetics in these two cell types presented sustained protein levels until $96 \mathrm{~h}$ post-transfection (Figure 12), the proteasome-independent CIITA degradation does not seem to significantly contribute to the post-translational regulation of CIITA. In contrast, the combined treatment of HeLa cells reconstituted the CIITA levels (Figure 13B), indicating that the transient CIITA expression in this cell line (Figure 12) is the result of an intense proteasome-mediated degradation. Combined, these results indicate that the CIITA is a target of both proteasome-dependent and -independent proteolytic mechanisms. However, the cell type-specific CIITA protein levels specifically reflect the differential regulation of the proteasome-mediated CIITA degradation by the cell lines. This is the first time that the CIITA post-translational regulation by proteasomal degradation is directly compared between different human cell types.

\subsection{Evaluation of PML proteins expression by non-immune cells}

The suggestion that the interaction with PML isoform II at the PML nuclear bodies protects CIITA from proteasomal degradation ${ }^{82}$, led us to test whether the transfected cell lines differ in the PML isoform expression. As shown in Figure 14, antiPML polyclonal antibody detected a major signal of around $130 \mathrm{kDa}$ that corresponded to isoforms I and II of PML ${ }^{178,179}$ in all cell lines, revealing no association between the PML II expression kinetics (Figure 14) and the CIITA kinetics previously detected 
(Figure 12). Intriguingly, the presumed Small Ubiquitin-like Modifier (SUMO)modified species of PML ${ }^{178,179}$ were also detected in the three cell lines (Figure 14) and particularly the mono-SUMOylated form of PMLs (Figure 14, indicated by an asterisk) was present in a cell type-specific manner, matching the CIITA expression pattern (Figure 12). First, the constant presence of the mono-SUMOylated PMLs in HEK293 cells corresponded to the sustained high levels of CIITA expression (Figure 12) and the lack of proteasomal degradation (Figure 13B). Second, the mono-SUMOylated PMLs detection only at 12 and $24 \mathrm{~h}$ post-transfection of HeLa cells coincided with the highest levels of CIITA expression, followed by the rapid proteasomal degradation in the absence of the SUMO-modified PMLs (Figures 14, 12 and 13B). Lastly, the appearance of this PML form $24 \mathrm{~h}$ post-transfection of Huh-7 cells (Figure 14) paralleled with an increase in the CIITA levels (Figure 12). 


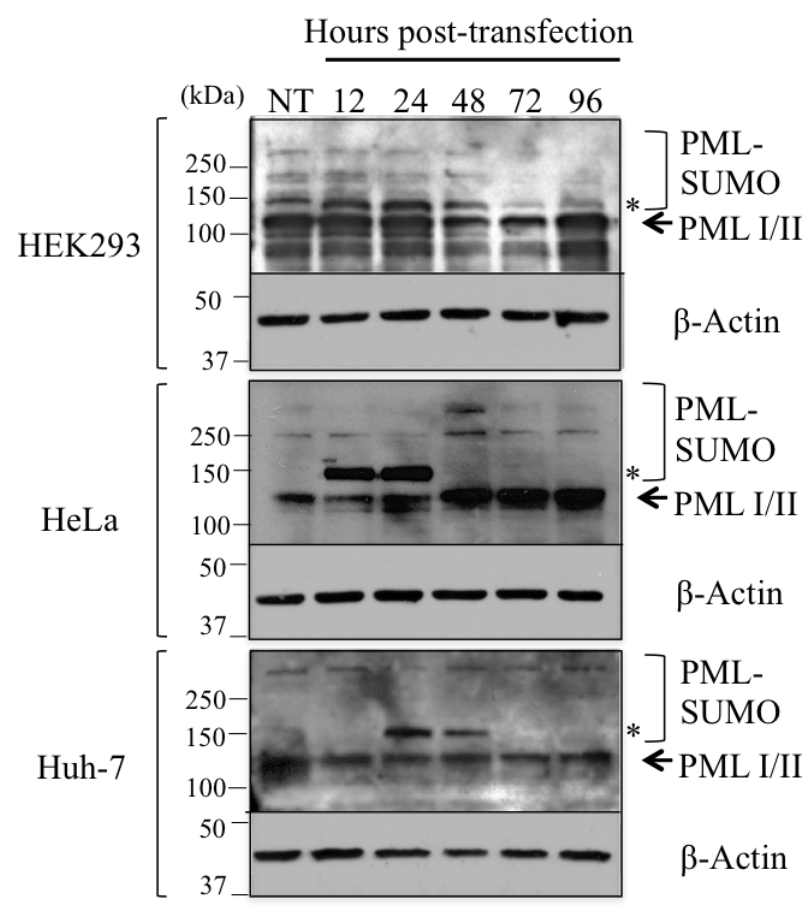

Figure $14-$ p/CIITA-transfected cells differentially regulate the PML monoSUMOylation. HEK293, HeLa and Huh-7 cells were either p/CIITA transfected or not transfected and harvested at the indicated times, lysed and $15 \mu \mathrm{g}$ of each cell extract were transferred to PVDF membranes. The membranes produced for each cell line were analyzed in parallel by western blot for the PML expression using the same film exposure time. The allocation of PML isoforms I/II and SUMOylated isoforms was performed as previously described ${ }^{178}$. An asterisk indicates the mono-SUMOylated PMLs. $\beta$-actin was used as a loading control. Numbers on the left indicate the positions of standard protein marker bands.

Since only the SUMOylated PML is able to assemble the PML nuclear bodies $^{180,181}$, where the CIITA interaction with PML II and protection from proteasomal degradation were shown to take place ${ }^{82}$, these data indicate that human non-immune cells differentially regulate the PML mono-SUMOylation as a mechanism to control CIITA susceptibility to proteasomal degradation. Future studies will investigate the mechanisms by which the PML mono-SUMOylation is differentially regulated by transfected cell lines. 


\subsection{CIITA protein expression in p/CIITA-transfected mice cells}

The unraveled cell type-specific regulation of CIITA stability has a direct impact on the CIITA transactivation activity and must be considered during the development and evaluation of strategies to overexpress the CIITA for adjuvant purposes. However, bearing in mind that all transfected non-immune cells expressed a transcriptionally active CIITA protein and underwent sustained MHC II molecules expression, albeit with varying efficiencies, we considered the plasmid-based delivery system a suitable tool for further investigations of CIITA potential as a molecular adjuvant.

The following step in the evaluation of the p/CIITA construct includes future studies in a mouse model that were enabled by the detection of CIITA protein expression in p/CIITA-transfected NIH/3T3 mouse cells (Figure 15A), which underwent MHC II molecules expression $48 \mathrm{~h}$ post-transfection (Figure 15B). In collaboration with Dr. Bruno Douradinha (Center for Vaccine Research, University of Pittsburgh, USA), combined immunizations of mice with $\mathrm{p} / \mathrm{CIITA}$ and a DNA vaccine against the Dengue virus will address the impact of CIITA expression in the vaccine potency. 
A

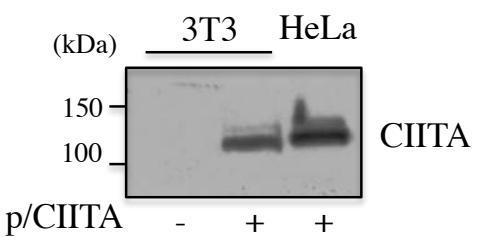

B

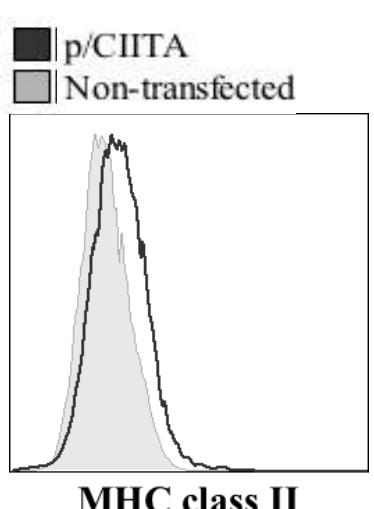

Figure 15 - p/CIITA-transfected mice cells are able to express the transcriptionally active human CIITA protein. NIH3T3 mice cells were transfected with p/CIITA and harvested $48 \mathrm{~h}$ post-transfection. (A) Analysis of CIITA expression. Transfected cells were lysed and $100 \mu \mathrm{g}$ of cell extract was transferred to PVDF membrane for CIITA expression analysis by western blot. Non-transfected cells were used as a negative control and p/CIITA-transfected HeLa cells (50 $\mu \mathrm{g}$ ) were used as positive control for the CIITA expression. The detection of $\beta$-actin was used as a loading control. Numbers on the left indicate the positions of standard protein marker bands. Results shown are representative of one experiment. (B) Analysis of MHC II induction. $\mathrm{p} / \mathrm{CIITA}$-transfected cells were analyzed according to the I-A/I-E surface expression by flow cytometry. The population of interest was defined by exclusion of dead cells and doublets. Nontransfected cells were used to set the gate determining positive cells. Histogram shows the comparison of MFIs from non-transfected cells and p/CIITA transfectants. Results shown are representative of two experiments.

\subsection{CIITA gene delivery to human dendritic cells}

So far we had restricted our analysis of CIITA expression and activity in transfected human cell lines mainly due to technical simplicity and low cost. Once we confirmed that our sequence design strategy could promote the expression of a transcriptionally active CIITA protein in three different human cell types, we aimed the validation of these findings in a primary human cell model. Considering that our research group headed by Prof. Alberto Duarte is primarily focused on the cell-based immunotherapy against HIV-1, using human monocyte-derived dendritic cells pulsed with the AT-2 inactivated autologous virus, we reasoned to transfect mo-DCs with the optimized CIITA sequence. This strategy would provide not only a primary cell system 
to analyze the CIITA expression and activity, but also an immunotherapy model in which we would be able to assess the impact of CIITA overexpression in the immunogenicity of the in vitro produced dendritic cells.

\subsubsection{Establishment and characterization of DC cultures}

Several protocols have been developed for the in vitro differentiation of blood monocytes into iDCs and their maturation into different polarizing subtypes. Therefore it is possible to obtain DC- 1 cells capable of inducing Th1 response, DC-2 cells that induce Th2 response and DC-0 cells that promote $\mathrm{T}$ cell activation without polarizing the $\mathrm{T}$ helper response, among other subtypes. Here we established the differentiation of monocytes into these three mature DC subtypes. Since mDCs pulsed with AT-2 inactivated HIV-1 can be functionally deviated to induce a suppressive immune response $^{150,151}$, we reasoned to compare DCs transfected with the designed CIITA sequence and matured into these different subtypes in order to evaluate whether the CIITA overexpression would be able to counteract the virus-mediated functional bias regardless of the maturation protocol. In addition, this strategy would provides us with the singular opportunity to evaluate how the CIITA overexpression would affect the polarizing activity of each mDC subtype, and select the transfected DC subtype able to trigger the most effective Th1 response against HIV-1.

The DC differentiation cultures were established according to protocols developed by Dr. Robbie Mailliard (University of Pittsburgh, USA). Monocytes from four healthy individuals were differentiated into iDCs after a 5-days culture with the cytokines IL-4 and GM-CSF. Mature DCs were generated by stimulation for two 
additional days with subtype-specific cytokines cocktails. After seven days of culture, the cells presenting size and granularity characteristic of DCs were evaluated by flow cytometry for the expression of CD14, CD11c, HLA-DR, CD80, CD83 and CD40, all surface molecules indicative of DCs differentiation and maturation (Figures 16 and 17). As expected, the complete differentiation from monocytes was evidenced by the absence of the CD14 molecules (monocyte marker) and an enhanced CD11c expression (myeloid DCs marker) in almost $100 \%$ of the cells analyzed (Figure 17B), accompanied by an elevated HLA-DR expression (Figures 16 and 17C). The average viability was approximately $97 \%$ as determined by staining with Live/Dead Viability/Cytotoxicity Kit (Figure 17A). The mDC subtypes presented an enhanced expression of HLA-DR, CD83, CD80 and CD40 in comparison with iDCs, indicating an effective maturation (Figure 16 and 17C). Not surprisingly, the mDC subtypes presented few differences regarding to the surface molecules expression, insufficient for distinction of each subtype by phenotypic characteristics (Figure 16 and 17C). 


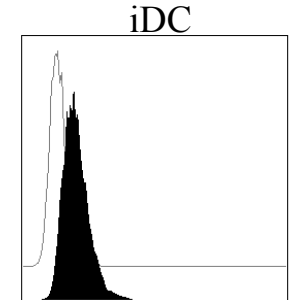

CD14

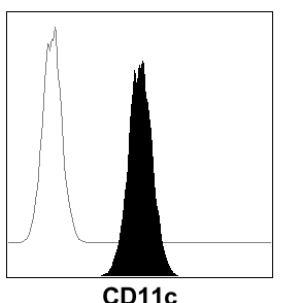

CD11c

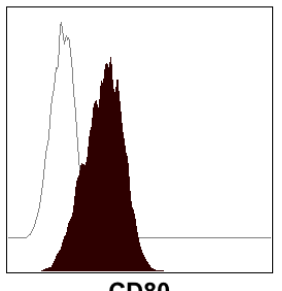

CD80

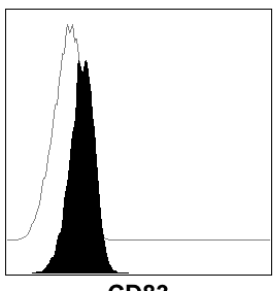

CD83
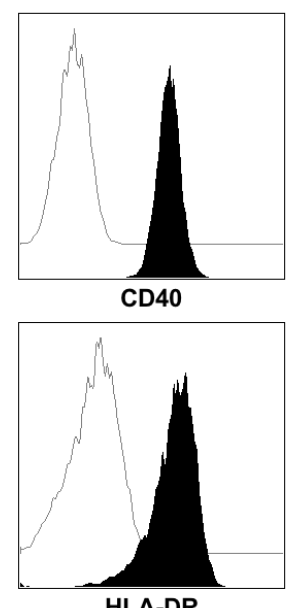

DC-0

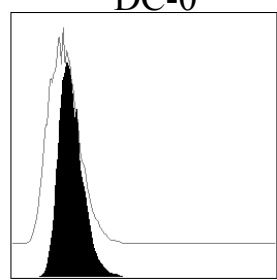

CD14

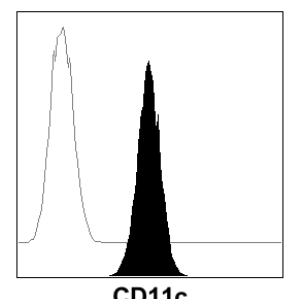

CD11c

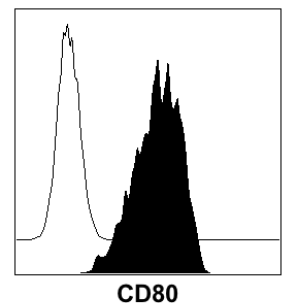

CD80

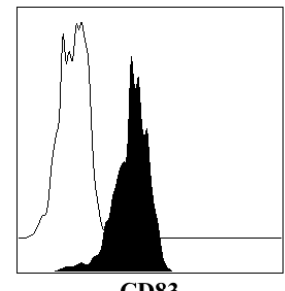

CD83
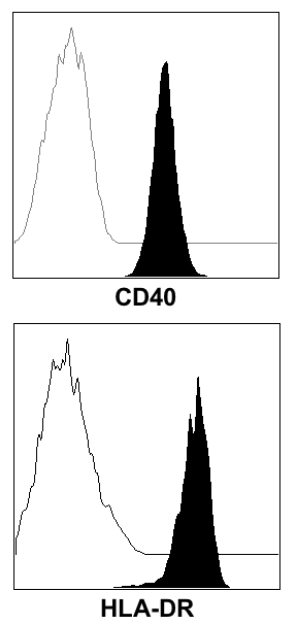

HLA-DR

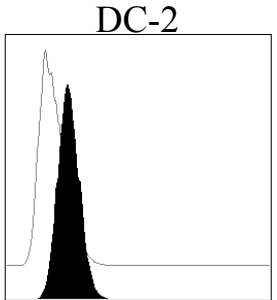

CD14

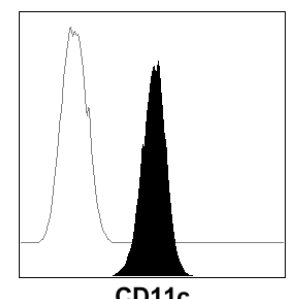

CD11c

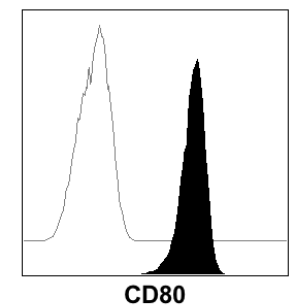

CD80

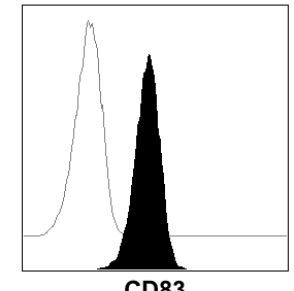

CD83
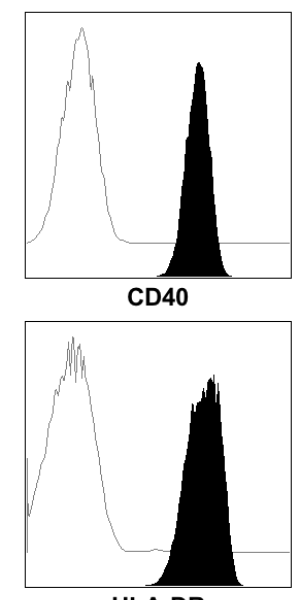

HLA-DR

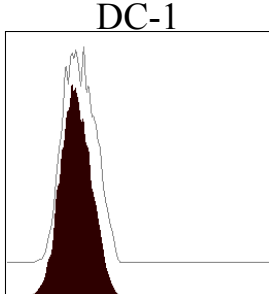

CD14
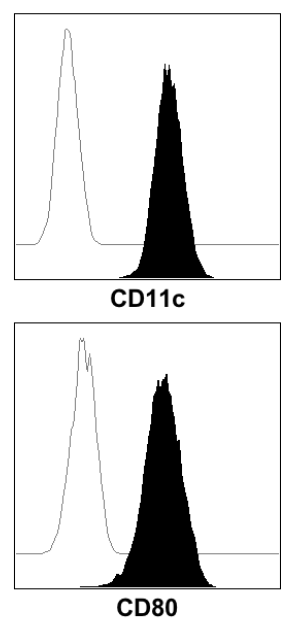

CD80

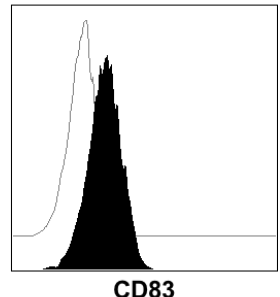

CD83
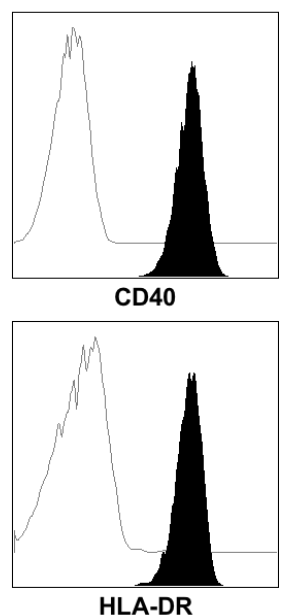

Figure 16 - Phenotypic characterization of monocyte-derived dendritic cells. Monocytes from healthy individuals were cultured for five days to differentiate into immature dendritic cells (iDCs) followed by maturation during two additional days into the subtypes DC-0, DC-2 and DC-1. Histograms represent the MFIs of CD14, CD11c, CD80, CD83, CD40 and HLA-DR expressed on the surface of iDCs and each subtype of mDCs, analyzed by flow cytometry. Analyses were performed in the population of $\mathrm{CD} 14^{-} \mathrm{CD} 11 \mathrm{c}^{+}$cells after exclusion of doublets and dead cells. Data representative of 4 independent experiments. 
A

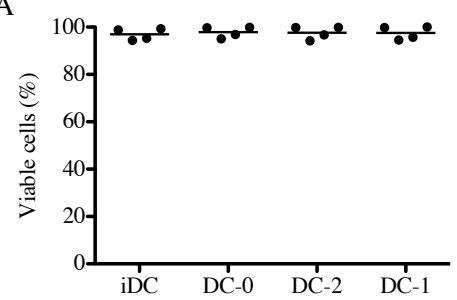

B

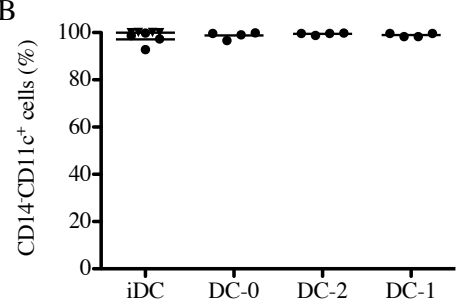

$\mathrm{C}$
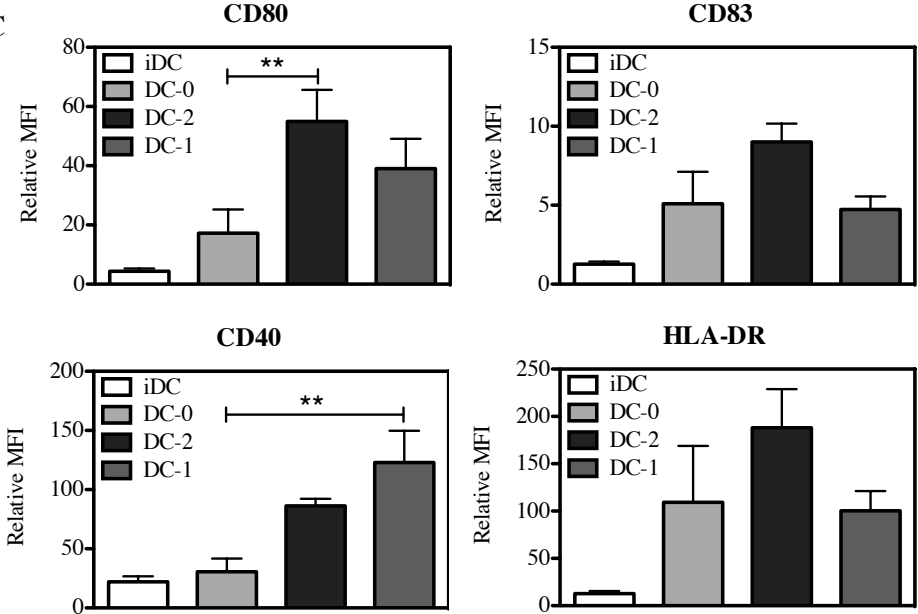

HLA-DR

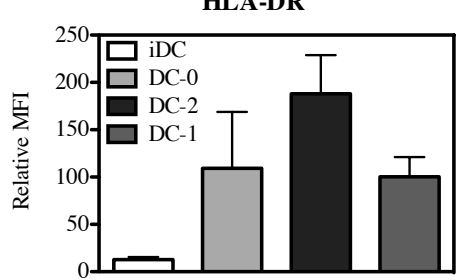

Figure 17 - Mature dendritic cell subtypes are phenotypically similar. iDCs, DC-0, DC-2 and DC-1 generated from healthy donors were analyzed and compared for viability and surface markers expression by flow cytometry. (A) Chart representing the percentage of viable DCs determined by the exclusion of dead cells stained with the Live/Dead Viability/Cytotoxicity kit, after exclusion of doublets. (B) Chart representing the percentage of differentiated DCs determined as $\mathrm{CD} 14^{-} \mathrm{CD} 11 \mathrm{c}^{+}$after the exclusion of dead cells and doublets. Each plot represents one donor (A-B). (C) Bar graphs display the relative MFIs of the indicated surface molecules. Analyses were performed in the population of $\mathrm{CD} 14^{-} \mathrm{CD} 11 \mathrm{c}^{+}$cells after exclusion of doublets and dead cells. The relative MFIs were calculated by dividing experimental sample MFI with isotype control MFI. Data are means \pm SEM of four independent experiments. ${ }^{* *}: \mathrm{p}<0.01$.

It was previously shown that the polarizing subtypes of mDCs are morphologically and phenotypically similar, but they differ greatly in reticulation and IL-12(p70) secretion once activated by $\mathrm{CD}^{+} \mathrm{T}$ helper cells expressing CD40L ${ }^{129,130,182}$. Thus, we confirmed the proper maturation of iDCs into DC-0, DC- 1 and DC-2 by assessing their ability to produce IL-12(p70), a cytokine that mediates Th1 immune responses after DC activation.

The three mDC subtypes and iDCs were then co-cultured with J558 cells constitutively expressing the CD40L molecule, which interacts with the CD40 expressed by DCs and triggers cell activation. After 48 hours, the culture supernatants were collected and the IL-12(p70) production was quantified by ELISA. Our results 
confirmed the successful differential maturation of DCs, showing cytokine levels in the culture supernatant of each subtype compatible with their respective immune response polarizing activity ${ }^{129}$. In this regard, non-activated mature cells produced undetectable levels of IL-12(p70) while all activated mature cells presented cytokine production. The DC-2 cells presented the lowest cytokine levels, followed by iDCs and DC-0, both producing less the $5 \mathrm{pg} / \mathrm{mL}$ of IL-12(p70). Disparately, DC-1 presented very high levels of cytokine, producing about $10 \mathrm{pg} / \mathrm{mL}$ of IL-12(p70) (Figure 18).

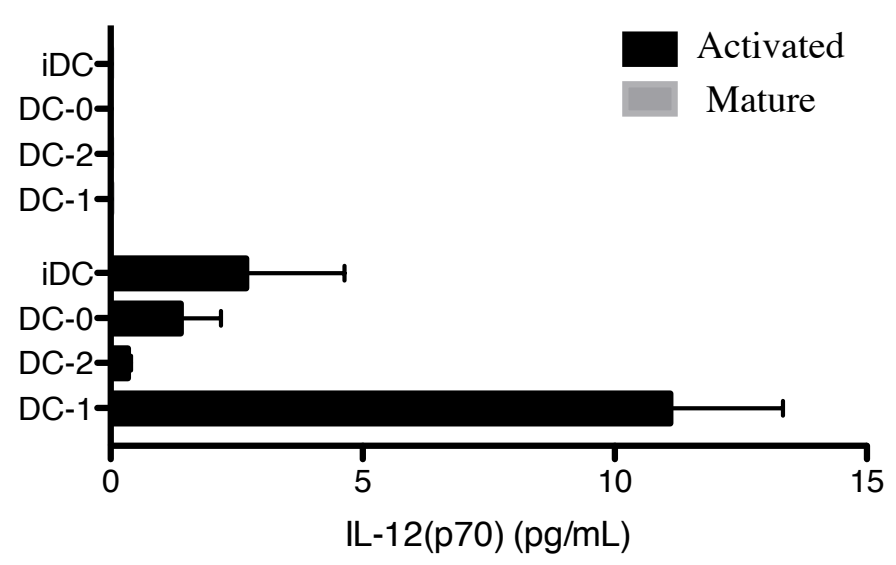

Figure 18 - Activated dendritic cell subtypes significantly differ in the secretion of IL12(p70). iDCs, DC-0, DC-2 and DC-1 generated from healthy donors were activated through the co-culture with J558 cells stably expressing rhu CD40L (activated). Cells cultured in parallel without J558 were used as negative controls (mature). Bar graph represents the IL12(p70) production by each DC subtype, measured in the cultures supernatants by ELISA. Data are means \pm SEM of four independent experiments.

Collectively, the phenotypic and functional analysis of in vitro generated moDCs demonstrated the successful establishment of DC differentiation cultures to be used in transfection assays for CIITA expression. 


\subsubsection{Expression of endogenous CIITA protein by DCs}

In order to determine the levels of endogenous CIITA expression by in vitro differentiated DCs, we performed western blot assays using cell lysates from iDCs and the three mDC subtypes generated from healthy individuals (Figure 19). The cell lysate from HEK293 cells transfected with p/CIITA was used as a positive control for CIITA expression. Interestingly, none of the analyzed DCs presented CIITA expression levels detectable by western blot technique, contrasting with the strong protein expression by the transfected cell line (Figure 19). The lack of CIITA expression in mDCs is in agreement with previous studies showing inhibition of CIITA gene transcription during the maturation process ${ }^{21,165}$. Conversely, iDCs are known to express CIITA, and the unexpected undetectable CIITA levels in these cells indicates extremely low levels of endogenous CIITA expression by iDCs, as reported for other human cells. In fact, even after induction by IFN- $\gamma$, the endogenous CIITA expression by fibroblast cell lines could only be detected after immunoprecipitating $650 \mu \mathrm{g}$ of total cell extract ${ }^{23}$.

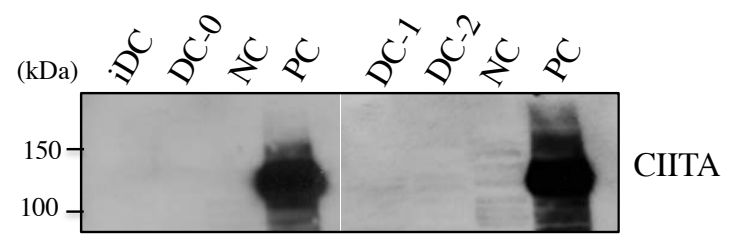

Figure 19 - Expression of endogenous CIITA protein by iDCs and mDC subtypes. iDCs, DC-0, DC-1 and DC-2 generated from healthy donors were harvested after 7 days of culture and used for cell lysate preparation. $70 \mu \mathrm{g}$ of each cell extract were transferred to PVDF membranes for CIITA expression analysis by western-blot. NC: non-transfected HEK293 cells (30 $\mu \mathrm{g})$; PC: $\mathrm{p} /$ CIITA-transfected HEK293 cells $(30 \mu \mathrm{g})$. $\beta$-actin was used as a loading control. Numbers on the left indicate the positions of standard protein marker bands. Results are representative of three independent experiments. 


\subsubsection{Development of CIITA delivery systems}

\subsubsection{1 mRNA electroporation}

The optimized CIITA sequence was delivered to human cell lines through cationic lipid-mediated transfection of a plasmid DNA (p/CIITA). Unfortunately, this very same methodology could not be applied to human dendritic cells, which are extremely resistant to transfection with exogenous $\mathrm{DNA}^{183}$. In order to achieve the efficient delivery of the same optimized CIITA sequence to DCs, we initially attempted to develop an mRNA electroporation system.

First of all, we produced DNA constructs containing the T7 RNA polymerase promoter to be used as templates for the in vitro transcription of mRNA molecules. The template for the CIITA-encoded mRNA transcription was generated by inserting the designed CIITA sequence into the pMACS plasmid (Figure 20A). The pMACS-CIITA construct presented the sequence of CIITA factor followed by an IRES sequence and the LNGFR gene (Figure 20A). Transcripts derived from this plasmid would consist of a bicistronic mRNAs whose translation in DCs would allow the concurrent and independent expression of CIITA and LNGFR surface marker (CD271), a membrane protein with a truncated cytoplasmic tail that is not normally expressed in dendritic cells.

This strategy would allow the isolation of LNGFR-expressing cells by the MACSelect kit (Miltenyi Biotech) to obtain samples containing only DCs efficiently expressing CIITA, for subsequent analysis. DCs electroporated with mRNA encoding only the LNGFR gene, transcribed from the empty pMACS plasmid (Figure 20C), 
would be used as a negative control consisting of cell samples subjected to the same process of electroporation and positive selection but not expressing CIITA. We also produced a pMACS-eGFP construct (Figure 20B) for the synthesis of an eGFPencoding mRNA to be used in the establishment of the best conditions for DCs electroporation assays.

A pMACS-CIITA construct

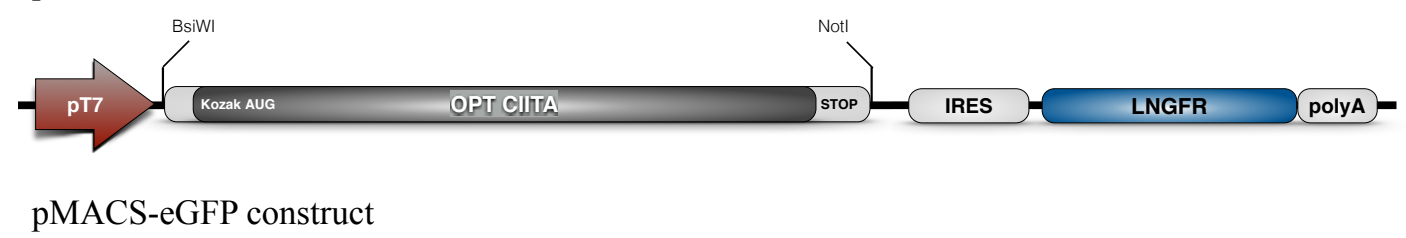

B pMACS-eGFP construct

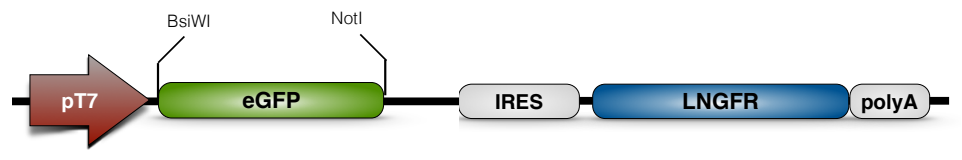

C pMACS plasmid

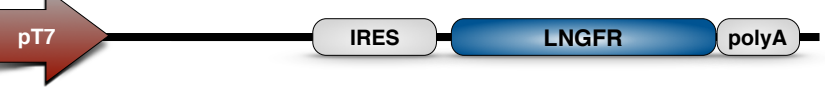

Figure 20 - pMACS constructs used for in vitro mRNA synthesis. (A) Schematic representation of pMACS-CIITA construct presenting the OPT CIITA sequence at 3 '-end of the T7 promoter and upstream of the IRES-LNGFR sequences. (B) Illustration of pMACS-eGFP construct presenting the eGFP sequence at 3 '-end of the T7 promoter and upstream of the IRESLNGFR sequences. (C) Representation of the empty pMACS plasmid.

Next, the mRNAs encoding eGFP and LNGFR were successfully produced through in vitro transcription of pMACS-eGFP and pMACS, previously linearized by cleavage at the Bstz17L restriction site, situated at the 3 -end of the polyA tail from the LNGFR gene. The in vitro transcriptions resulted in a high yield of capped mRNAs (approximately $50 \mu \mathrm{g}$ per reaction), the integrity of which was certified by denaturing agarose gel showing sharp bands and the absence of smears (Figure 21A). The eGFP mRNA functionality was confirmed by the transfection of HEK293 cells using 
Lipofectamine 2000, and detection of eGFP-positive cells 24 and $48 \mathrm{~h}$ post-transfection by flow cytometry (Figure 21B).

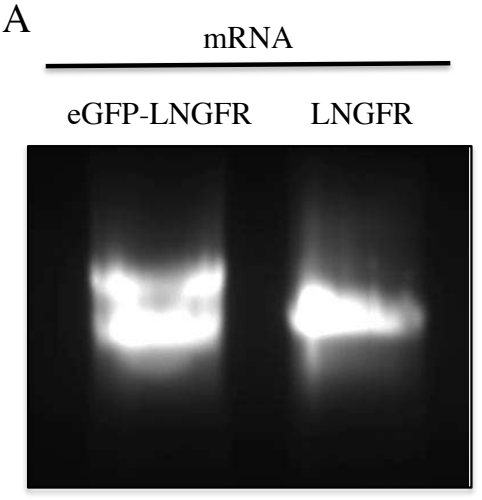

B

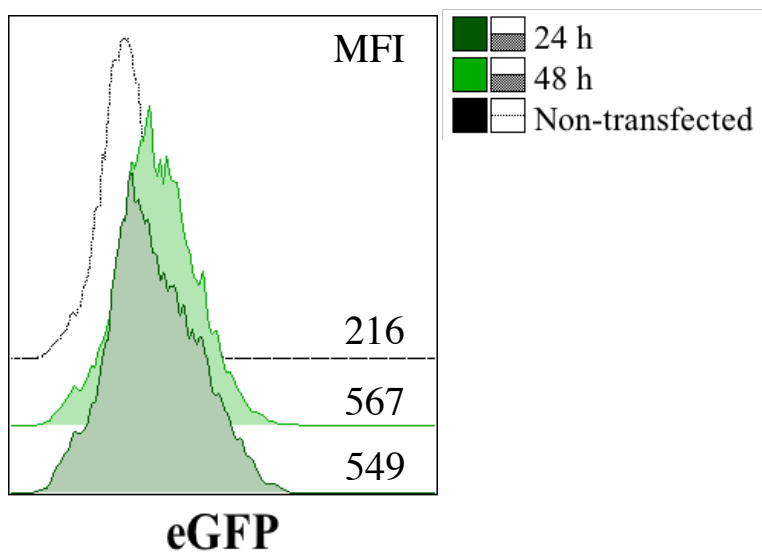

Figure 21 - In vitro transcription of eGFP and LNGFR mRNAs. (A) Denaturing agarose gel demonstrating the integrity of mRNAs. A high yield of eGFP and LNGFR mRNAs was obtained through the in vitro transcription of linearized pMACS-eGFP and pMACs constructs, respectively. The single-stranded mRNAs sharp bands were visualized with ethidium bromide, indicating a lack of RNA degradation. (B) eGFP expression by HEK293 cells transfected with eGFP mRNA. Cells were transfected with eGFP mRNAs and harvested 24 and $48 \mathrm{~h}$ posttransfection for analysis of eGFP expression by flow cytometry. The population of interest was defined by exclusion of dead cells and doublets. Histograms show the comparison between eGFP MFIs of mRNAs transfectants and non-transfected cells. Numbers in the histograms represent the absolute MFI of each sample. Results shown are representative of two experiments.

Despite the efficient synthesis of mRNAs encoding eGFP and LNGFR, the CIITA mRNA production was hampered by difficulties in linearizing the pMACSCIITA vector. The plasmid DNA linearization with a restriction enzyme downstream of the sequence to be transcribed is of utmost importance for in vitro transcription to avoid the generation of extremely long and heterogeneous RNA transcripts. Similar to the assays with pMACS and pMACS-eGFP, the first attempt to linearize pMACS-CIITA was performed using the Bstz17L restriction enzyme, which was not efficient at cleaving the plasmid even after several variations in the digestion parameters for assay optimization (Figure 22A, first panel). Despite the few options of restriction sites located at the 3'-end of the LNGFR polyA tail, three other enzymes were unsuccessfully 
tested to linearize the vector (PsiI, MfeI and SalI - Figure 22A, other panels). Taking into account that the same restriction enzymes efficiently cleaved the empty pMACS plasmid (Figure 22A, middle panel), it is reasonable that the long CIITA sequence could have induced an increased DNA condensation, leading to the blockage of restriction enzymes interaction with their respective cleavage sites.

We next decided to use the PvuI enzyme, whose single cleavage site was situated in the plasmid backbone, at about $2 \mathrm{~kb}$ downstream of the sequence-of-interest. However, the PvuI restriction assays yielded a low molecular weight fragment indicating unspecific cleavage (Figure 22B, left panel). The following PvuI digestions performed with the pcDNA3.1-CIITA plasmid resulted in the same band pattern (Figure 22B, right panel). Considering that the pcDNA3.1-CIITA encodes the same OPT CIITA sequence and also presents a single PvuI restriction site located at the plasmid backbone, we concluded that the PvuI enzyme was probably cleaving an unspecific site in the CIITA sequence, precluding the use of this enzyme for pMACS-CIITA linearization.

Due to the impossibility of linearizing the pMACS-CIITA plasmid by restriction enzymes, we alternatively performed PCR assays as a last attempt to generate the linear fragment to be used as a template for CIITA mRNA in vitro transcription. Thus, primers were designed to amplify a 5,631 bp fragment comprising the $\mathrm{T} 7$ promoter necessary for transcription initiation, the CIITA insert and the IRES-LNGFR sequence, as well as the polyA tail. Unexpectedly, despite attempts at optimizing the PCR assay, the fragment obtained presented approximately $3 \mathrm{~kb}$, not corresponding to the expected fragment (Figure 22C). Therefore, despite all efforts, it was not possible to synthesize the CIITA mRNA due to difficulties in obtaining a suitable DNA template for in vitro 
transcription. The insertion of CIITA into the pMACS vector seems to induce a DNA condensation, which impairs proper digestion and PCR amplification.

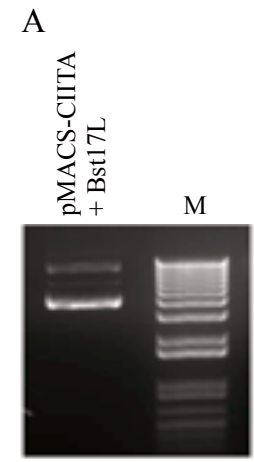

B

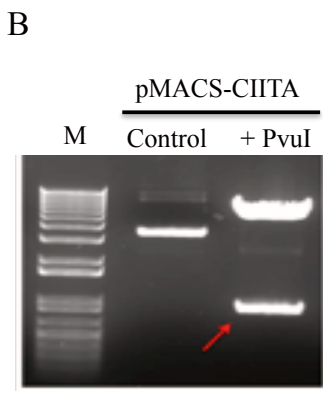

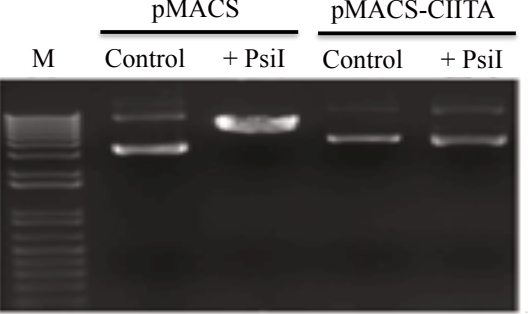

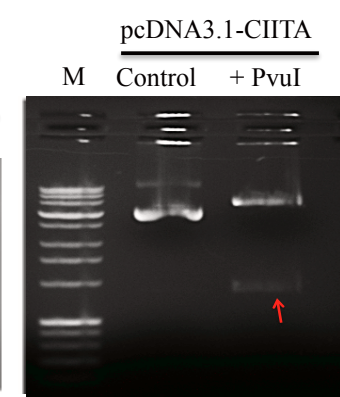

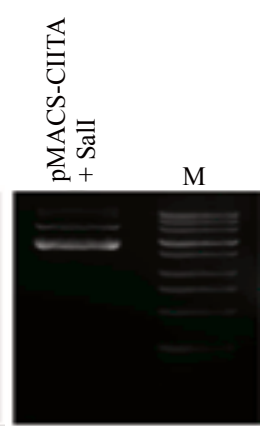

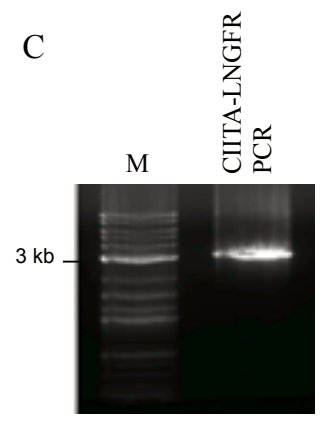

Figure 22 - Attempts to obtain a linear template for CIITA mRNA transcription. (A) Agarose gel electrophoresis representing single digestions of pMACS-CIITA with four different enzymes that have only one restriction site at the polyA tail 3'-end. The pMACS vector was used as the positive control. (B) Left: single digestion of pMACS-CIITA with PvuI enzyme, which has only one restriction site at 2,000 bp downstream of the polyA tail. Right: the digestion of pcDNA3.1-CIITA was used as the positive control since it presents only one restriction site for PvuI in the plasmid backbone. Red arrows indicate fragments generated by unspecific digestion at the CIITA sequence. (C) PCR amplification of a linear template using the pMACS-CIITA as a template. The agarose gel electrophoresis shows the generation of an unspecific amplicon of approximately 3,000 bp, while the expected amplicon should contain 5,631 bp comprising T7 promoter, CIITA insert, IRES-LNGFR sequence and the polyA tail. M: E-Gel ${ }^{8} 1 \mathrm{~kb}$ Plus DNA Ladder or 2-Log DNA Ladder.

Parallel to the attempts to linearize pMACs-CIITA, the eGFP mRNA was used in preliminary assays for the establishment of dendritic cells electroporation. Monocytes obtained from a healthy individual were differentiated during 5 days into iDCs, which were electroporated through the application of square wave pulses. In this assay, cells were subjected to varied electric field strengths (300, 400, 500 and $600 \mathrm{~V}$ ) while maintaining fixed other electroporation parameters such as mRNA concentration, 
number of cells, cuvette type, number and length of pulses, according to Kavanagh et al. ${ }^{155} .48$ hours after electroporation, the iDCs were analyzed by flow cytometry for viability and eGFP expression in order to determine the electric field strength that promoted the lowest cell death and highest transfection efficiency (Figure 23).

Overall, the iDCs electroporation yielded high toxicity and inefficient transfection. All voltages tested resulted in low viability, with the mildest condition presenting $20.5 \%$ of viable cells $(300 \mathrm{~V})$. In regard to the transfection efficiency, the detection of eGFP positive cells was restricted to iDCs electroporated with 400 and 600 V, but representing only $2 \%$ of viable cells (Figure 23 ).

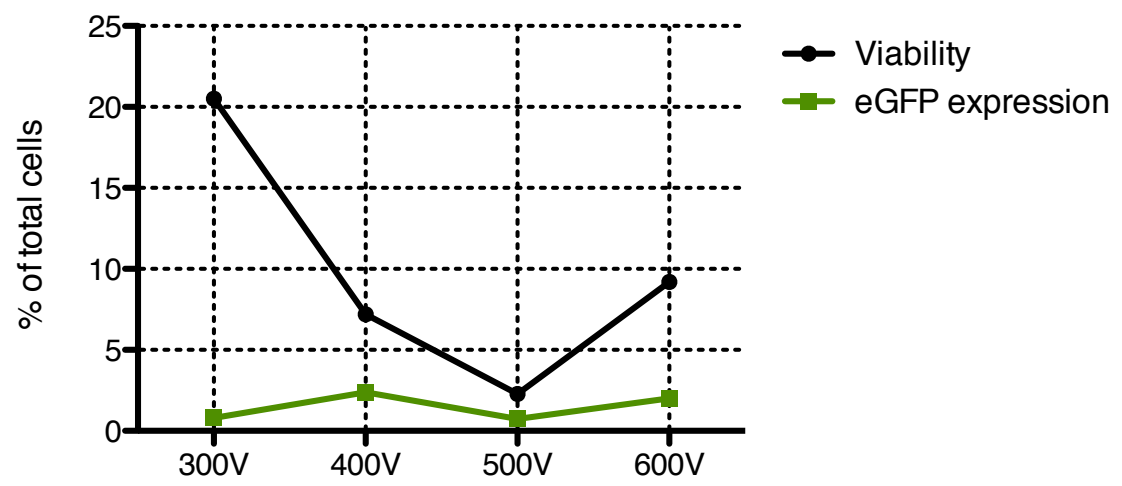

Figure 23 - eGFP expression and viability of immature dendritic cells electroporated with different electric forces. iDCs generated from one healthy donor were harvested after 5 days of culture and electroporated with the eGFP mRNA. Square wave electroporation fixed conditions were: pulse length of $0.075 \mathrm{msec}, 2 \mathrm{~mm}$ cuvette, $10 \mu \mathrm{g}$ mRNA per $10^{6}$ cells. Variable electric fields were applied with voltages ranging from 300 to $600 \mathrm{~V}$. Electroporated cells were cultured without cytokines for $48 \mathrm{~h}$ and analyzed by flow cytometry for viability and eGFP expression. The percentage of viable DCs was determined by the exclusion of dead cells stained with the Live/Dead Viability/Cytotoxicity kit after exclusion of doublets. Mock controls were used to set the gate determining positive cells for eGFP expression after exclusion of doublets and dead cells. Chart represents the percentage of viable cells and eGFP-positive cells versus the applied voltages.

It is important to highlight that the mRNA encoding eGFP is about $2.5 \mathrm{~kb}$ smaller than the mRNA to be generated from the pMACS-CIITA-LNGFR, so the 
electroporation with CIITA mRNA is likely to be even more toxic and less efficient using the same conditions tested with eGFP mRNA. Despite the possibility to further optimize the protocol by varying other parameters such as mRNA concentration and electrical pulse length, the high toxicity of the electroporation method and the impossibility of producing CIITA mRNA led us to substitute the mRNA electroporation by the lentiviral transduction system.

\subsubsection{Lentiviral transduction}

The lentiviral gene delivery was chosen to replace the mRNA electroporation method based on previous reports showing efficient DCs transfection with low toxicity $^{184-186}$. Production of lentiviral vector particles is performed by transfecting plasmids harboring long terminal repeat (LTR) regions together with the gene-ofinterest in one vector (the transfer vector), while the additional transcripts required for packing (Gag/Pro/Pol) and encapsulation (Env) are encoded by separate packing and envelope vectors, respectively. In the present study, we first chose to use the envelope plasmid pMD2.G encoding the VSV-G envelope protein to produce pseudotyped lentiviral particles carrying the designed CIITA sequence encoded by the transfer vector SiEW (Figure 24). 
A SiEW-CIITA transfer vector

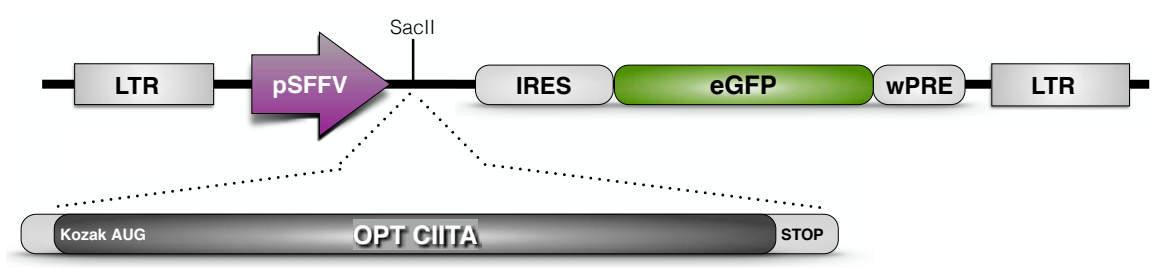

B Packing vector

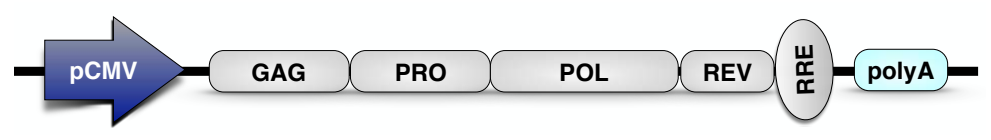

C Envelope vector

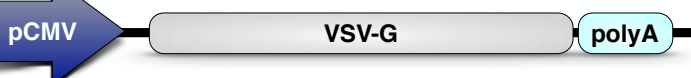

Figure 24 - Schematical representation of the lentiviral vector system for CIITA sequence delivery. (A) The transfer vector SiEW-CIITA produced by the insertion of OPT CIITA sequence into the self-inactivating lentivirus vector $\mathrm{SiEW}$, would allow the co-expression of CIITA and eGFP under the control of SFFV promoter. The recombinant transduction vector particles would be produced by co-transfecting SiEW-CIITA with a packing vector (B) and an envelope vector (C). LTR: Long terminal repeat; wPRE: post-transcriptional regulatory element; RRE: Rev response element.

The SiEW-CIITA construct was generated through the insertion of the CIITA sequence into $\mathrm{SiEW}$, and the different clones obtained were evaluated according to insert presence through PCR amplification and digestions with SacII (Figures 25A and B). Although these assays confirmed the insert presence and its proper size in the majority of tested clones, the assessment of insert orientation by digesting the same clones with the restriction enzymes XbaI (single site in the vector backbone) and BamHI (single site in the CIITA sequence) showed a pattern of several bands that did not correspond in size with the bands expected to be produced by clones containing the CIITA inserted in the correct or even inverted orientations (Figure 25C). These diagnostic digestion findings suggested either the cleavage of SiEW-CIITA clones at unspecific sites or the presence of extra restriction sites not described in the nucleotide sequence provided for the SiEW plasmid. 
As an alternative to assess the CIITA insert orientation, the SiEW-CIITA clones were sent for sequencing by Genscript using a forward primer specific to the SFFV promoter, located upstream of the CIITA insertion site. Unexpectedly, none of the clones could be sequenced due to the lack of amplification during the sequencing reaction, indicating no complementarity between the designed primer and the corresponding sequence in the vector (data not shown). Together, the diagnostic digestion and sequencing results strongly suggested that the SiEW plasmid nucleotide sequence, provided by Dr. Manuel Grez research group, does not correspond to the sequence of the plasmid used in the aforementioned experiments. The lentiviral plasmids are highly susceptible to homologous recombination through the LTRs sequences, thus the empty SiEW plasmid had possibly undergone sequence modifications during expansions in E. coli that were not checked and updated.

Since we were unable to identify which SiEW-CIITA clone contained the insert with proper orientation, we tried to select functional clones through evaluating their capacity to induce CIITA expression in human cells. Three SiEW-CIITA clones that were positive for the insert presence (Figure 25B and C) were used in the transfection of HEK293 cells, which were analyzed $48 \mathrm{~h}$ post-transfection by western blot. In this assay, none of the tested clones were able to induce detectable levels of CIITA protein (Figure 25E). In order to screen a larger number of clones in regard to the expression of CIITA, we transfected HEK293 cells with 20 different clones containing the CIITA sequence. $48 \mathrm{~h}$ after transfection, the expression of surface MHC II molecules was evaluated by flow cytometry as an indirect measurement of CIITA expression. None of the tested clones were capable of promoting CIITA expression and therefore were also incapable of expressing the MHC II. Also, the eGFP expression by the bicistronic vector (Figure 24C) was not detected (data not shown). These results revealed that the 
SiEW-CIITA clones were inefficient in expressing CIITA and eGFP, reinforcing our hypothesis that the empty SiEW vector was intensely modified by recombination.
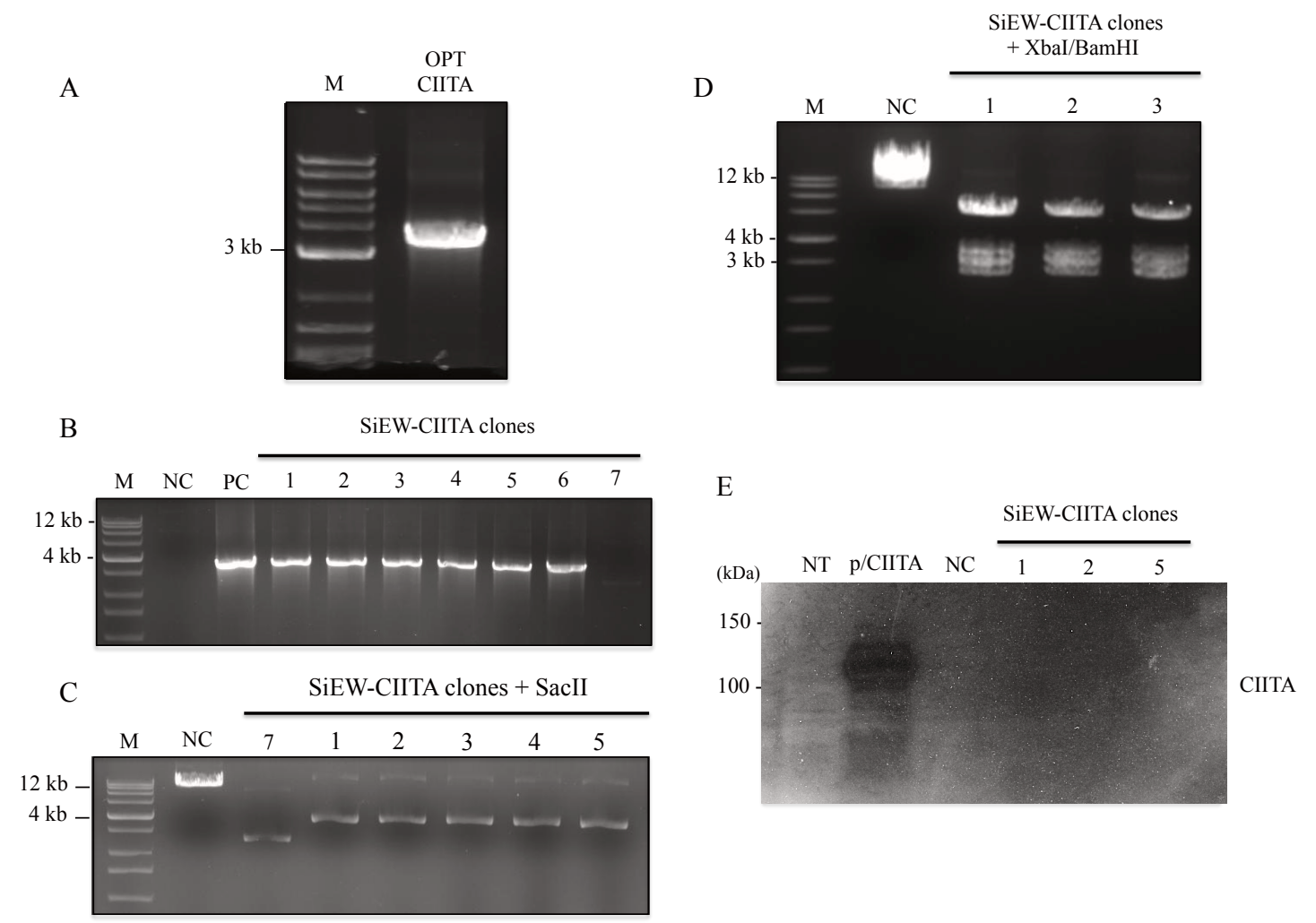

Figure 25 - SiEW-CIITA vector construction. (A) Confirmatory PCR amplification of the OPT CIITA sequence from the SiEW-CIITA clones. NC: negative control - SiEW; PC: positive control - commercial vector encoding OPT CIITA_2A. M: Perfect DNA Ladder (Novagen) (B) Restriction analysis of SiEW-CIITA clones with the enzyme SacII. The band migrating at approximately 3,415 bp corresponds to OPT CIITA insert. NC: negative control - SiEW; M: Perfect DNA Ladder. (C) Restriction analysis of insert orientation in SiEW-CIITA clones with the enzymes XbaI and BamHI. The agarose gel shows the generation of several unspecific bands. NC: negative control SiEW; M: Perfect DNA Ladder. (D) Western-blot analysis of CIITA expression by HEK293 cells transfected with SiEW-CIITA clones. Transfected cells were harvested $48 \mathrm{~h}$ post-transfection, lysed and $50 \mu \mathrm{g}$ of each cell extract was transferred to PVDF membrane for CIITA expression analysis. Non-transfected cells (NT) and SiEW transfectants $(\mathrm{NC})$ were used as negative controls, while p/CIITA transfectants (p/CIITA) were used as positive control for the CIITA expression. Numbers on the left indicate the positions of standard protein marker bands.

Considering the difficulties in obtaining a functional SiEW-CIITA construct, we decided to purchase a new lentiviral transfer vector directly from the manufacturer to avoid possible homologous recombination and obtain a reliable nucleotide sequence. Hence, the LeGO-iG2 plasmid (Addgene) was acquired. Similar to SiEW, this 
commercial plasmid is a self-inactivating lentiviral vector presenting the SFFV promoter and the IRES-eGFP sequence, and can be used in the production of viral particles in association with the same packing and envelope plasmids that would be used with $\mathrm{SiEW}$. Other advantages are the vector's smaller size and the presence of MCS, which facilitate cloning strategies.

The L/CIITA vector was generated through the insertion of the designed CIITA sequence into LeGO-iG2 (Figure 26A). This transfer vector was used for the production of LV-CIITA lentiviral particles, which carried the CIITA sequence linked to the eGFP marker gene through an IRES region. We also produced a lentiviral vector carrying only the eGFP marker gene, named LV-control, to be used as the negative control of transduction assays. For the production of LV-control particles, the L/eGFP2 transfer vector was constructed by the insertion of a second eGFP sequence into the LeGO-iG2 MCS (Figure 26B). Even though the plasmid LeGO-Ig2 already carries one eGFP sequence controlled by IRES, this protein is not efficiently expressed in the absence of a cDNA inserted before IRES. Therefore the insertion of another eGFP sequence was the chosen strategy to obtain LV-control particles able to promote an efficient eGFP expression.

Both L/CIITA and L/eGFP2 were verified according to the inserts presence and orientation by PCR assays and diagnostic digestion. The L/CIITA capacity to induce CIITA protein expression was additionally validated by western blot assays using lysates processed 48 hours after HEK293 cells transfection with the transfer vector (Figure 26C). Non-transfected cells and L/eGFP2 transfectants were used as negative controls for CIITA expression. 
A L/CIITA transfer vector

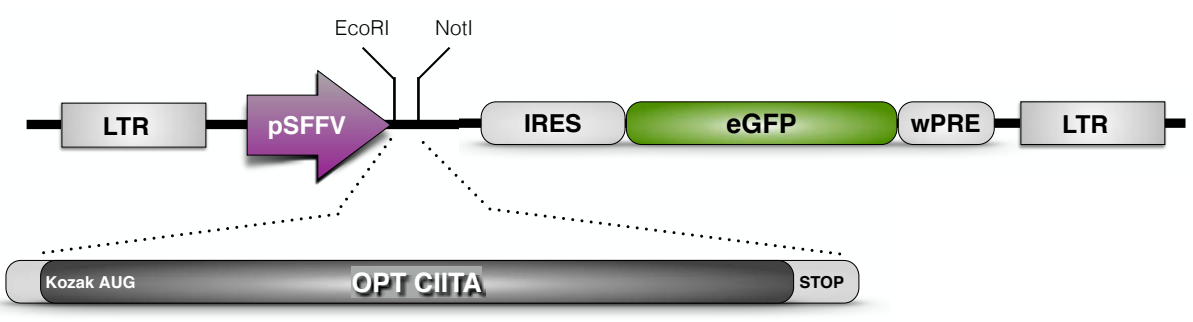

B L/eGFP2 transfer vector

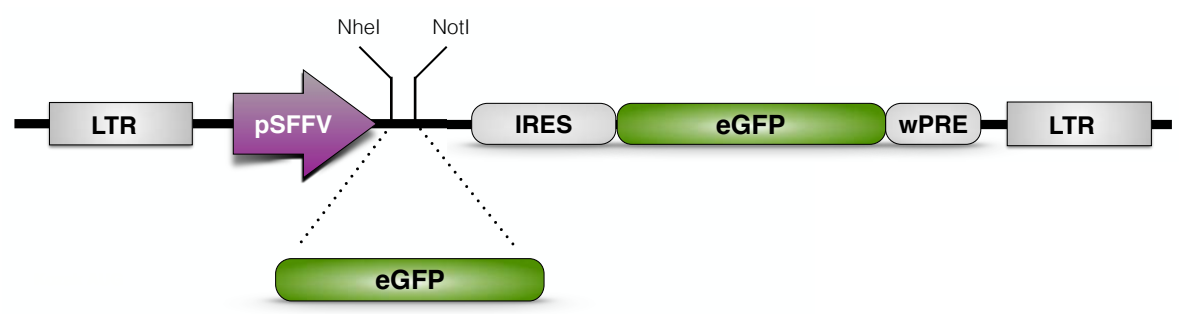

$\mathrm{C}$

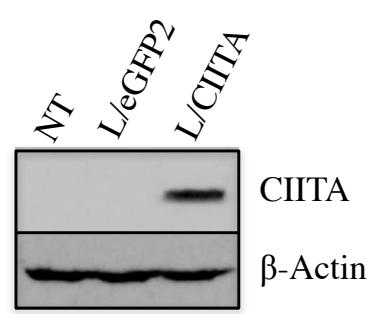

Figure 26 - Transfer vectors construction using the LeGO-iG2 plasmid. (A) Schematic representation of the L/CIITA transfer vector produced by the insertion of OPT CIITA sequence into the Lentiviral Gene Ontology vector LeGO-iG2. L/CIITA allows the co-expression of CIITA and eGFP under the control of an SFFV promoter. (B) Illustration of the L/eGFP2 transfer vector by the insertion of eGFP sequence into LeGO-iG2. The control vector L/eGFP2 allows the intense expression of eGFP molecules. LTR: Long terminal repeat; wPRE: posttranscriptional regulatory element. (C) Western-blot analysis of CIITA expression by HEK293 cells transfected with L/CIITA vector. Transfected cells were harvested $48 \mathrm{~h}$ post-transfection, lysed and $50 \mu \mathrm{g}$ of cell extract was transferred to PVDF membrane for CIITA expression analysis. Non-transfected cells (NT) and L/eGFP2 transfectants were used as negative controls. $\beta$-actin was used as a loading control.

Subsequently, the lentiviral vector particles were produced in cultures of cells co-transfected with the constructed transfer vectors, along with packing and envelope vectors. All plasmids used were endotoxin-free purified. For the optimization of viral particles production, several tests were performed to compare parameters such as cell line (293T or 293FT), cells confluence at the time of transfection, plasmids concentration, transfection method (cationic lipids or calcium-phosphate), packing 
vector (pCMVAR8.91 or psPAX2), intervals for harvest of viral-containing supernatants and viral stocks concentration procedure. The lentiviral vector preparations were titrated in $293 \mathrm{~T}$ cells to identify the method yielding the highest titers of LVCIITA (Section 3.10), which was subsequently used for the large-scale production of LV-CIITA and LV-control. Every viral stock prepared was titrated in 293T cells for normalization to comparable titers and to assure the reproducibility of the methodology established.

\subsubsection{Lentiviral transduction of human cell lines}

Prior to DC transduction assays, we tested the LV-CIITA transduction efficiency in human cell lines. HEK293, Huh-7 and HeLa cells were transduced with LV-CIITA and the expression of eGFP and MHC II molecules was analyzed $72 \mathrm{~h}$ posttransduction by flow cytometry. All tested cell lines presented high albeit variable transduction efficiencies, represented by the proportion of cells expressing eGFP (Figure 27A, B and C). Remarkably, HEK293 cells were more efficiently transduced, presenting about $90 \%$ of eGFP positive cells. These data, in addition to the detection of surface and intracellular MHC II molecules in all transduced cell lines (Figure 27), indicate that the LV-CIITA lentiviral vector is a valuable tool for the optimized CIITA sequence delivery to human cells, providing higher transfection rates than the previously established p/CIITA delivery system.

The produced LV-CIITA lentivirus is also advantageous in comparison to the p/CIITA DNA construct due to the co-expression of the eGFP marker, which facilitates the detection and/or isolation of transduced cells for the investigation of CIITA activity. 
Thus, in the aforementioned transduction assays, the expression of MHC II molecules was analyzed within the eGFP-positive cells population (Figure 27A, B and C). As expected, HEK293 cells showed the highest levels of MHC II molecules, followed by an intermediary expression in HeLa and very low MHC II levels in Huh-7 cells (Figure 27D). These findings corroborate with the pattern of the MHC II expression previously found in p/CIITA transfectants (Figure 10), further supporting our mechanism of cellspecific post-translational regulation of CIITA factor activity. 
A HEK293
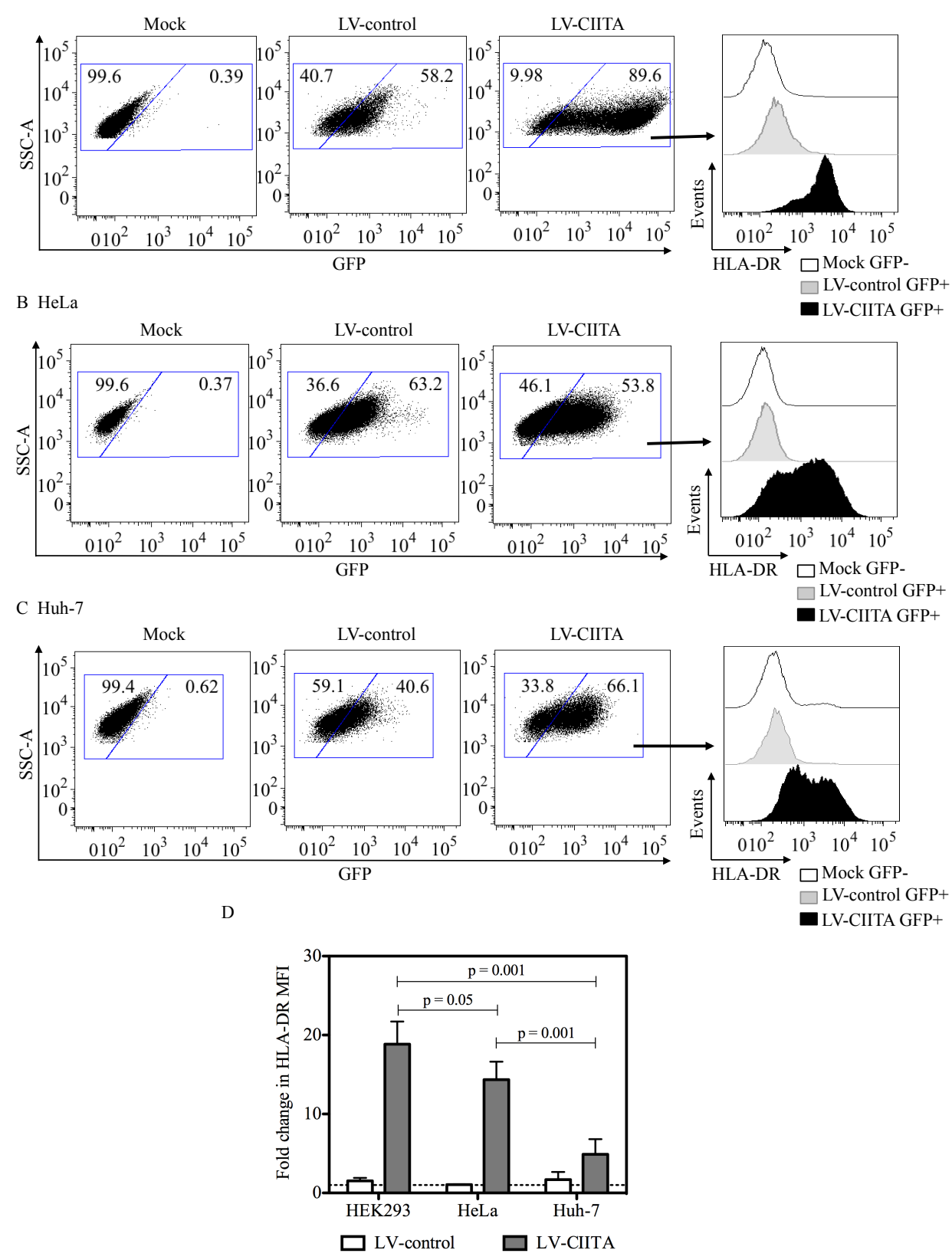

Figure 27 - LV-CIITA-transduced cell lines express MHC II molecules at distinct levels and kinetics. HEK293 (A), HeLa (B) and Huh-7 (C) cells were transduced with a lentiviral vector carrying the designed CIITA sequence and eGFP marker gene (LV-CIITA) or a lentiviral vector carrying only eGFP (LV-control), both at an MOI of 2. Cells were harvested $72 \mathrm{~h}$ posttransduction for analysis of HLA-DR intracellular and surface expression by flow cytometry. Dead cells and doublets were excluded from analysis and the HLA-DR expression was determined within the eGFP-positive population. Mock-transduced cells were used to set the gate determining eGFP-positive cells. Numbers in the plots represent the frequency of eGFPnegative cells (left gate) and eGFP-positive cells (right gate). The HLA-DR median intensity of fluorescence (MFI) is shown in histograms (A-C) and in bar graphs (D). The fold change in MFI was calculated by dividing the HLA-DR MFI units of the eGFP-positive population from LV-transduced cells by the HLA-DR MFI units of the eGFP-negative population from mocktransduced cells. Data are mean \pm SEM of three independent experiments. $\mathrm{P}$ values are indicated. 


\subsubsection{Lentiviral transduction of DCs}

The LV-CIITA lentivirus was then used for the optimized CIITA sequence delivery to dendritic cells. The transduction of monocyte-derived dendritic cells may be performed in three distinct moments during the in vitro differentiation culture: (i) in purified monocytes, before adding IL-4 and GM-CSF to promote DC differentiation; (ii) in iDCs obtained after 5 days of culture, before the addition of maturation cocktails; (iii) in mDCs obtained after 7 days of culture. In the present study, transduction assays were performed in the monocytes followed by differentiation into DCs. This methodology was chosen to avoid excessive manipulation of the very sensitive dendritic cell cultures, which could lead to unspecific phenotypic and functional alterations. In addition, the transduction of monocytes with LV-CIITA would enable CIITA expression and activation of target genes transcription long before the introduction of maturation stimulus to the culture, known to downregulate CIITA activity through increased protein export from the nuclei ${ }^{86}$. Thus, this strategy was considered the most promising for the induction of phenotypic and functional changes in transduced DCs.

The lentiviral transduction efficiency was then tested in monocytes derived from three healthy individuals, which were transduced with LV-CIITA and LV-control, followed by the differentiation into iDCs. In these tests, the DCs were maintained in the immature stage until the seventh day of differentiation, when the cells were analyzed. The analysis of cells morphology by optical microscopy revealed drastic alterations only in the cells transduced with LV-CIITA, which presented increased long surface projections and attachment to the plate, accompanied by the loss of a round shape prevalent in LV-control transduced cells and non-transduced iDCs (Figure 28A). These morphological changes were indicative of increased cells maturation induced by the 
LV-CIITA transduction. Accordingly, the LV-CIITA-transduced iDCs presented significantly higher levels of the synthetic CIITA mRNA compared to LV-control- and mock-transduced iDCs, which presented very low levels of the endogenous CIITA mRNA, comparable to the basal levels of non-transduced iDCs (Figure 28B). The high mRNA levels detected seven days after transduction strongly indicated the effective transduction by the developed lentiviral system. Intriguingly, parallel flow cytometry analysis showed insignificant levels of eGFP positive cells in both LV-CIITA and LVcontrol transductants (data not presented).

The transduced cells were also analyzed by flow cytometry for the intracellular and surface expression of HLA-DR molecules as an indirect evaluation of CIITA protein expression. The intracellular staining was performed to allow the detection of newly synthesized proteins induced by CIITA, since iDCs are known to efficiently express MHC II molecules while maintaining part of the produced proteins in intracellular vesicles ${ }^{21,89}$. The analysis restricted to the $\mathrm{CD} 14^{-} \mathrm{CD} 11 \mathrm{c}^{+}$cell population revealed an unspecific increase in HLA-DR expression stimulated by the lentiviral vector, since both LV-CIITA and LV-control transductants presented significantly higher protein levels than non-transduced iDCs (Figure 28C). Surprisingly, there was no significant difference between the HLA-DR levels of LV-CIITA and LV-control transductants (Figure 28D), regardless of the abundant amount of CIITA mRNA production by the first group of transduced cells.

The flow cytometric analysis of other DCs surface markers indicated the occurrence of a transduction-triggered DCs maturation, evidenced by the increased levels of CD80 and CD83 in cells transduced with the control lentivirus (Figure 28E), similar to the pattern found for HLA-DR expression (Figure 28C). Interestingly, the 
LV-control virus triggered a stronger expression of DCs surface molecules than LV-

CIITA, suggesting higher transduction efficiency by LV-control in these cells.

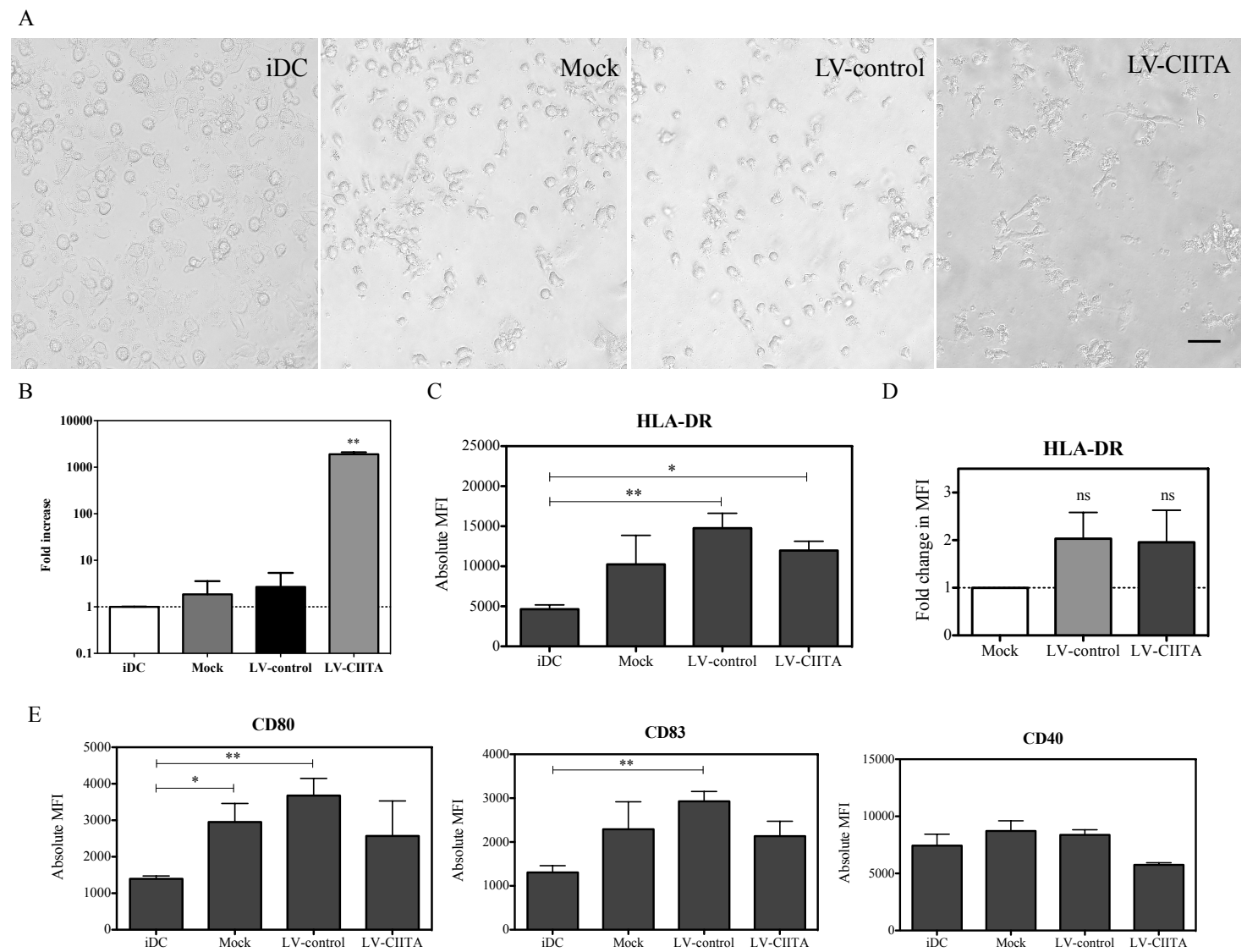

Figure 28 - The lentiviral vector LV-CIITA efficiently transduces monocytes from healthy individuals without significant CIITA-specific induction of MHC II molecules in iDCs. Monocytes isolated from PBMCs of healthy individuals were transduced with the lentiviral vectors LV-CIITA or LV-control, both at an MOI of 2. One day after transduction, the monocytes were cultured in the presence of GM-CSF and IL-4 for 7 days to differentiate into iDCs. Non-transduced (iDC) and mock-transduced iDCs (mock) were used as negative controls. (A) Optical microscopy images of iDC cultures at day 7, showing important morphological changes induced by LV-CIITA transduction. Scale bar, $5 \mathrm{~cm}$. (B) CIITA mRNA levels in transduced iDCs. RNA from non-transduced iDCs, mock-transduced and LV-transduced cells obtained at day 7 were analyzed by qRT-PCR for the expression of CIITA. RNA expression was normalized to $\beta$-actin RNA, and the fold increase was determined by dividing the expression in each sample by that of iDCs. Data are mean \pm SD of three independent experiments. ${ }^{* *}, \mathrm{p}=0.0034$. (C-E) At day 7, iDCs were analyzed for the expression of DC surface markers by flow cytometry. Dead cells and doublets were excluded from the analysis, and iDCs were defined as $\mathrm{CD} 14^{-} \mathrm{CD} 11 \mathrm{c}^{+}$. (C) Bar graph displaying the absolute MFIs of surface and intracellular HLA-DR molecules. (D) Effect of LV transduction on HLA-DR expression. Bar graph representing the fold changes in HLA-DR MFIs from LV-transduced iDCs relative to the mock-transduced iDCs. (E) Bar graph displaying the absolute MFIs of the indicated surface molecules. Data are means \pm SEM of three independent experiments. ${ }^{* *}, \mathrm{p}<0.01 ;^{*}, \mathrm{p}<0.05$. 
Our research group currently runs dendritic cells immunotherapy clinical trials for HIV-1 infection in humans, so one of our final goals is to investigate if the CIITA overexpression in DCs from HIV+ individuals would improve the immunotherapy potency. Therefore, we also tested the lentiviral transduction efficiency in monocytes derived from three individuals chronically infected with HIV (Figure 29).

Overall, the findings were very similar to the results obtained with dendritic cells from healthy individuals. The LV-CIITA-transduced iDCs presented significantly higher levels of CIITA mRNA compared to the controls (Figure 29B), even though flow cytometric analysis could not detect eGFP-expressing cells. Then the HLA-DR expression, as well as DCs surface markers expression, was enhanced by the transduction method, with the strongest induction of cells maturation by the LV-control vector (Figures 29C and D). Finally, the expression of MHC II molecules did not differ between LV-CIITA and LV-control transductants (Figure 29C). Curiously, the DCs morphology after transduction with LV-CIITA indicated certain level of maturation, but the LV-control-transduced cells presented more intense modifications, with long surface projections and elongated shape (Figure 29A). 
A

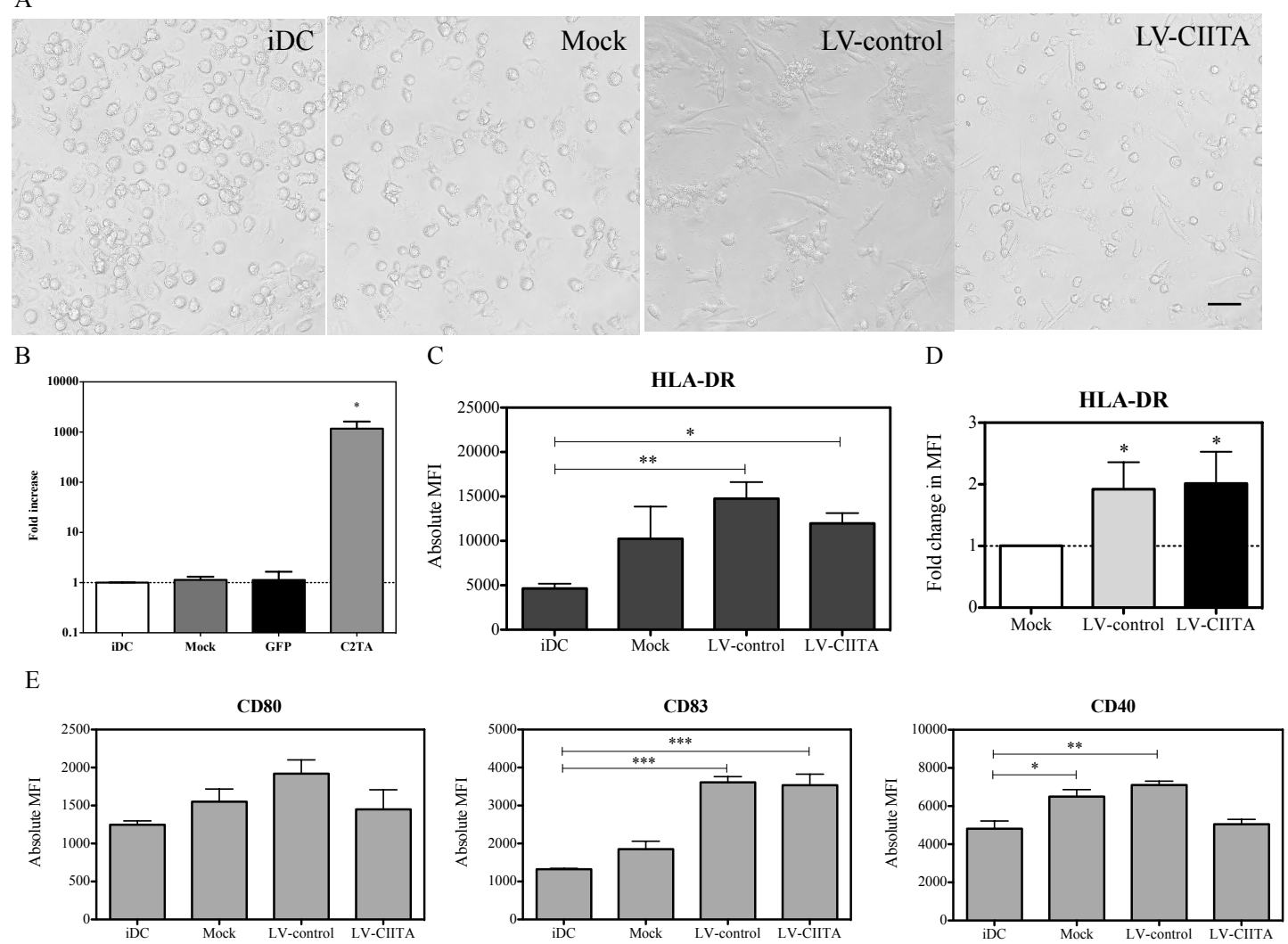

Figure 29 - The lentiviral vector LV-CIITA efficiently transduces monocytes from HIVpositive individuals without significant CIITA-specific induction of MHC II molecules in iDCs. Monocytes isolated from PBMCs of HIV positive donors were transduced with the lentiviral vectors LV-CIITA or LV-control, both at an MOI of 2. One day after transduction, the monocytes were cultured in the presence of GM-CSF and IL-4 for 7 days to differentiate into iDCs. Non-transduced (iDC) and mock-transduced iDCs (mock) were used as negative controls. (A) Optical microscopy images of iDC cultures at day 7, showing morphological changes induced by LV-control and LV-CIITA transductions. Scale bar, $5 \mathrm{~cm}$. (B) CIITA mRNA levels in transduced iDCs. RNA from non-transduced iDCs, mock-transduced and LV-transduced cells obtained at day 7 were analyzed by qRT-PCR for the CIITA expression. RNA expression was normalized to $\beta$-actin RNA, and the fold increase was determined by dividing the expression in each sample by that of iDCs. Data are mean \pm SD of three independent experiments. ${ }^{*}, \mathrm{p}=$ 0.0467. (C-E) At day 7, iDCs were analyzed for the expression of DC surface markers by flow cytometry. Dead cells and doublets were excluded from analysis, and iDCs were defined as CD14 ${ }^{-} \mathrm{CD} 11 \mathrm{c}^{+}$. (C) Bar graph displaying the absolute MFIs of surface and intracellular HLADR molecules. (D) Effect of LV transduction on HLA-DR expression. Bar graph representing the fold changes in HLA-DR MFIs from LV-transduced iDCs relative to the mock-transduced iDCs. (E) Bar graph displaying the absolute MFIs of the indicated surface molecules. Data are means + SEM of three independent experiments. ${ }^{* * *}, \mathrm{p}<0.001 ; * *, \mathrm{p}<0.01{ }^{*}, \mathrm{p}<0.05$.

Altogether the transduction assays using dendritic cells from healthy and HIV+ individuals indicate that the lentiviral delivery system developed here is an effective tool to mediate the designed CIITA sequence delivery to these primary human cells. 
However, by themselves the viral particles induced a potent maturation of transduced DCs, which constituted a drawback for the indirect evaluation of the CIITA protein expression through the analysis of MHC II transactivation. Further studies concerning the direct detection of CIITA protein expression in transduced cells are necessary to elucidate whether the DCs producing high levels of CIITA mRNA are able to express the protein or if these cells present a more restricted post-translational regulation of CIITA activity than the previously investigated human cell lines.

Interestingly, the comparison of PML proteins expression by non-transduced monocytes, iDCs and HEK293 cells by western blot, demonstrated that monocytes from healthy individuals present approximately 30\% less mono-SUMOylated PMLs than HEK293 cells (Figure 30). Aditionally, the monocytes differentiation into iDCs leads to a downregulation of this SUMO-modified form, resulting in cells presenting about $60 \%$ less mono-SUMOylated PMLs than the analyzed cell line. Similar protein levels were observed in iDCs derived from HIV+ donors (Figure 30). Unfortunately, the limited amount of monocytes form HIV+ patients impaired the evaluation of this group of cells.

Taking into account the mechanism of post-translational regulation of CIITA activity identified in the present study, where the levels of mono-SUMOylated PMLs are associated with the CIITA protection from proteasomal degradation, the latest results suggest that the CIITA may be more efficiently degraded in human monocytes than in HEK293 cells, and this degradation is exacerbated during the differentiation into iDCs. Thus, the lack of increased MHC II expression in iDCs transduced with LVCIITA in the presence of high CIITA mRNA levels may be a result of CIITA protein increased degradation, as demonstrated before with HeLa cells. Again, further experiments directly detecting the CIITA protein expression in LV-CIITA transduced iDCs will be required to either confirm or refute this hypothesis. 

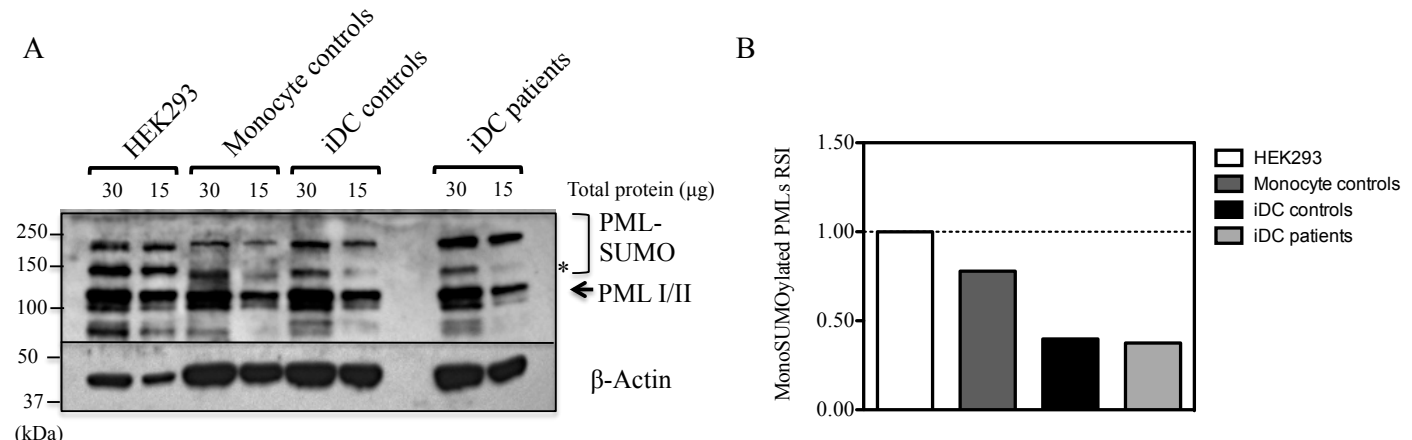

Figure 30 - Monocytes and iDCs express lower levels of mono-SUMOylated PML proteins than HEK293 cells. (A) Western-blot analysis of PML expression. Non-transfected HEK293 were cultured for $24 \mathrm{~h}$ and used for cell lysate preparation. Cryopreserved monocytes from three healthy donors (controls) were thawed and pooled for cell lysate preparation. iDCs generated from three healthy donors and three HIV positive patients were harvested after 7 days of culture, pooled per group of donors and lysed. 30 and $15 \mu \mathrm{g}$ of each cell extract were transferred to PVDF membrane to allow the comparison between PML levels of cell extracts by westernblot. The allocation of PML isoforms I/II and SUMOylated isoforms was performed as previously described ${ }^{178}$. An asterisk indicates the mono-SUMOylated PMLs. $\beta$-actin was used as a loading control. Numbers on the left indicate the positions of standard protein marker bands. (B) Quantitation of mono-SUMOylated PMLs levels. The mono-SUMOylated PMLs bands intensities as indicated by an asterisk in (A) were quantitated by densitometry and are displayed as relative signal intensity (RSI) per sample. Values were normalized to the band intensity of HEK293 cells.

\subsubsection{Lentiviral inoculation to human skin explant}

The development of a lentiviral system carrying the synthetic CIITA sequence enabled the beginning of a novel study aiming to inoculate the viral particles to human skin explants in order to identify the target primary human cells able to efficiently express the CIITA protein and promote antigen presentation and immune activation. This study has been recently initiated in collaboration with the doctoral candidate Parichat Duanghkae at the Center for Vaccine Research (University of Pittsburgh, USA), and had already generated promising preliminary data. The skin explant derived from one donor was inoculated with LV-CIITA or LV-control, and analyzed by immunofluorescence after $48 \mathrm{~h}$ of incubation to detect eGFP and CIITA expression (Figure 31). Skin sections obtained at the moment of inoculation $(0 \mathrm{~h})$ did not present 
detectable levels of endogenous CIITA expression. Sections from LV-control inoculations presented some cells expressing only eGFP after $48 \mathrm{~h}$ of incubation, while sections from LV-CIITA inoculations presented several dermal cells co-expressing CIITA and eGFP (arrows), with the transcription factor present in the nuclei. The LVCIITA induced a more efficient expression of CIITA than eGFP, since some cells presented only detectable CIITA expression (Figure 31). These findings suggest that the lentiviral delivery system developed here is able to transduce primary human cells in the skin, and efficiently induce the CIITA expression. The results obtained so far will be confirmed with skin explants from different donors and the dermal cells expressing the exogenous CIITA will be identified using antibodies to cell-specific markers and also evaluated according to the CIITA-mediated induction of MHC II expression. The expectation is that the data generated with this study will strengthen the LV-CIITA validation as a suitable molecular adjuvant for genetic immunizations. 

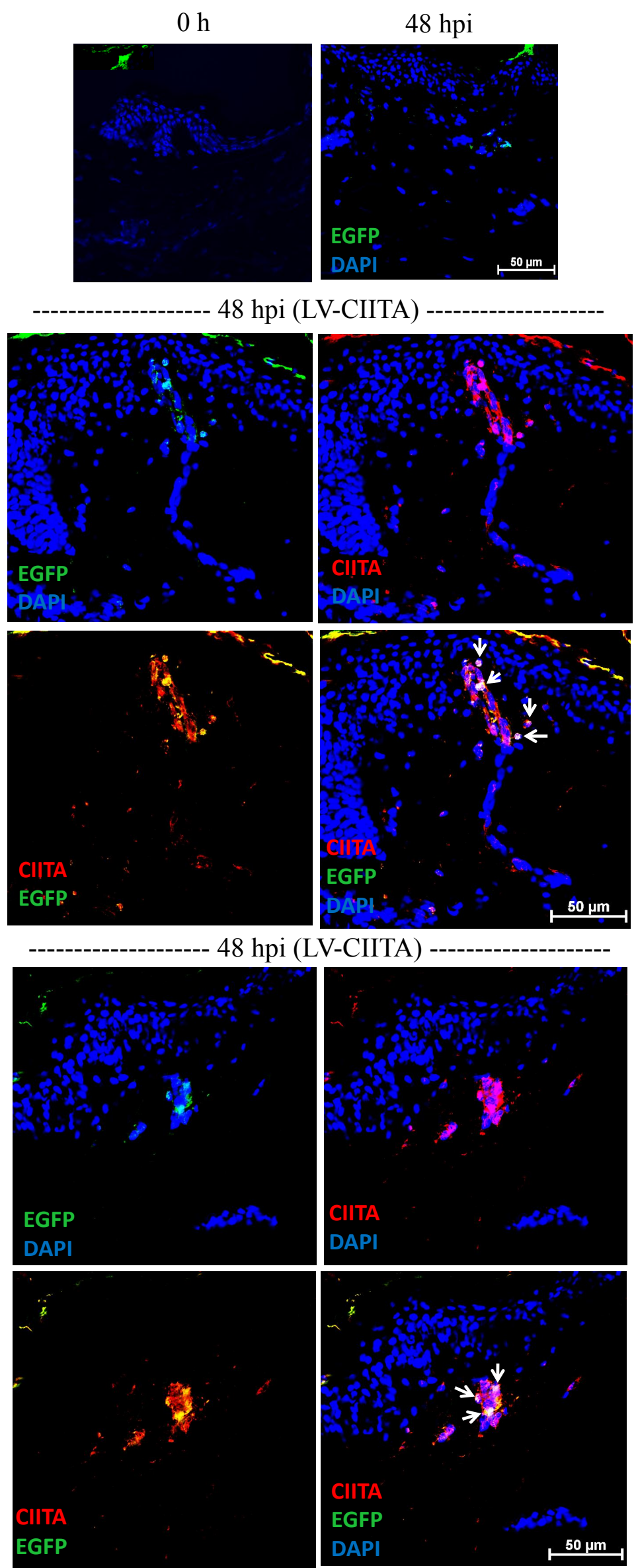
Figure 31 - The lentiviral vector LV-CIITA efficiently transduces primary human cells in skin explants and promotes CIITA expression. Skin explant was inoculated with $100 \mu \mathrm{L}$ of LV-CIITA or LV-control preparations at a titer of $2 \times 10^{7} \mathrm{TU} / \mathrm{mL}$ using a stabbing method with bifurcated needles. 0 and $48 \mathrm{~h}$ post-inoculation (hpi), skin sections $(6 \mu \mathrm{m})$ were stained for CIITA expression with anti-CIITA ab, followed by the secondary antibody conjugated with Alexa Fluor 549 (red). eGFP expression was also detected (green). Hoechst (blue) counterstains nuclei. Cells were analyzed by point scanning confocal microscopy (40X, 1.3 NA). Arrows indicate the CIITA and eGFP co-expression. Data are representative of one experiment. Scale bar, $50 \mu \mathrm{m}$. 
5 DISCUSSION 
The CIITA factor expression has been previously used in cell-based cancer vaccines to improve vaccine potency ${ }^{28,102,104-108}$, but the exact mechanism by which CIITA transfectants improve the immune response remains unknown, since the costimulatory molecules CD40, CD80 and CD86, necessary for efficient activation of T cells ${ }^{187}$, are not induced after CIITA expression in non-immune cells. Regardless, it was previously shown that CIITA-transfected cells act as surrogate APCs themselves ${ }^{25,97,106}$, but they may also be engulfed as necrotic and/or apoptotic bodies by DCs or produce MHC II-enriched exosomes ${ }^{188}$, in both cases providing DCs with preformed MHC-IIantigenic peptide complexes. Furthermore, depending on the transfected cell type, the CIITA mediated regulation of other immunologically relevant target genes, such as IL$4^{55,189}$, IL- $10^{51}$, plexin-A $1^{50}$, cathepsin $\mathrm{E}^{190}$ and FasL ${ }^{191}$, may synergistically potentiate the immune activation promoted by the increased antigen presentation.

Whatever the mechanism, it is evident that a correlation exists between adjuvant activity and CIITA expression ${ }^{28,108}$. So, in the present study, we aimed the development and validation of strategies to promote the efficient CIITA expression and activity in different human cells prior to the investigation of their immunological relevance as adjuvant systems.

Besides the well-established concept that the CIITA transcription can be induced upon IFN- $\gamma$ stimulation ${ }^{100,101}$, the coordination of different regulatory mechanisms and the consequential differences in the pattern of CIITA expression among distinct cell types are poorly understood. In an attempt to at least circumvent the reported regulatory mechanisms acting at the mRNA levels ${ }^{72}$ and potentiate translation efficiency, we designed a synthetic CIITA DNA sequence lacking cis-acting destabilizing elements and performed an artificial sequence optimization. In addition, the use of strong constitutive promoters avoided cell-specific epigenetic control of CIITA 
transcription $^{160,161,163-165}$. These strategies allowed intense mRNA production and efficient protein expression in three different non-immune cells, but discrepancies in the MHC II expression between transfected or transduced cell types, which corresponded with distinct levels and kinetics of CIITA expression, were indicative that additional post-translational mechanisms were maintaining the cell type-specific CIITA regulation.

Accordingly, the CIITA expression pattern in transfected cells suggested differences in the protein degradation between the three cell lines. Cycloheximide/MG132 treatment assays allowed the comparison of the proteins levels by reducing the influence of differences in mRNA levels or translational efficiency in the CIITA expression kinetics and confirmed that CIITA protein degradation is strikingly different between HEK293, Huh-7 and HeLa cells. These findings are in line with previous studies showing the modulation of the CIITA half-life as a means of manipulating its activity $^{77,79-81,166}$. Since CIITA is a known target for the ubiquitin-proteasome system ${ }^{73}$, we directly compared the influence of proteasomal activity in the CIITA degradation of the transfected cell lines to further investigate the regulatory mechanism responsible for the cell type-specific CIITA expression. Here we show for the first time that each cell type controls the CIITA expression stability by the differential modulation of CIITA susceptibility to proteasomal degradation. In this regard, the sustained CIITA basal levels detected in HEK293 and Huh-7 cells were shown to be refractory to further proteasomal degradation, while the transient CIITA expression by HeLa cells was related to rapid protein degradation by this proteolytic pathway.

Schanappauf et al..$^{73}$ previously established that CIITA poly-ubiquitination leads to protein degradation by the $26 \mathrm{~S}$ proteasome complex and mapped two destabilizing sequences (degron) in the CIITA protein that target it for ubiquitination and degradation. One of the degron sequences overlaps the acidic activation domain 
essential for CIITA transactivation and therefore cannot be mutated to improve protein stability without dampening the CIITA activity. This degron region is required for the ubiquitinated CIITA interaction with the 19S regulatory particle of the $26 \mathrm{~S}$ proteasome complex, which then recruits the $20 \mathrm{~S}$ proteolytic core necessary for protein degradation $^{81}$. To date, few mechanisms have been described as regulators of the proteasomal recruitment for CIITA degradation, but the protein post-translational modifications (PTMs) seem to play an important role. For instance, the CIITA phosphorylation at the Ser-280 is required for the E3 ligase recruitment, which ubiquitinate CIITA and target it for degradation ${ }^{77}$. In addition, Wu et al ${ }^{80}$ suggested that the dynamic regulation of CIITA acetylation by different deacetylases herald specific outcomes to the proteasome susceptibility, but the direct relation between CIITA deacetylation and proteasomal recruitment remains unclear. The latest study in the CIITA proteasomal degradation field indicated that the recruitment of CIITA to the PML nuclear bodies confers protection from proteasomal degradation ${ }^{82}$.

The PML bodies are dynamic structures present in the cell nuclei of almost all human tissues that among other functions, modulate the activities of several transcriptional regulation factors by PTMs ${ }^{192}$. The key organizers of PML bodies are the nuclear PML proteins ${ }^{193}$ that require the post-translational modification by SUMO (SUMOylation) to promote PML body assembly and subsequent recruitment of additional components ${ }^{180,181}$. Although the PML proteins present three SUMO conjugation sites ${ }^{194,195}$, it was previously shown that a single SUMOylation (monoSUMOylated PML) is required for the formation of PML bodies ${ }^{196}$. PML proteins and SUMO are inducible by IFN- $\gamma,-\alpha$ and $-\beta$, therefore these cytokines also induce PML body assembly ${ }^{197}$. 
Ulbricht et al. ${ }^{82}$ have determined that the CIITA recruitment to the PML bodies and consequent increased stability is mediated by protein-protein interactions with PML proteins. These analyses were performed in human cells overexpressing endogenous or exogenous CIITA and PML proteins, in which the PML II presented the strongest interaction with CIITA in the PML bodies, promoting the highest level of protein stability. Interestingly, our analysis of endogenous PML basal levels in transfected HEK293, HeLa and Huh-7 cells revealed a lack of association between the expression of PML II and the CIITA expression stability. On the other hand, the detection of mono-SUMOylated PML proteins perfectly matched the CIITA expression kinetics in HEK293 and HeLa cells, indicating that the modified PML proteins are protecting the CIITA from proteasomal degradation in these cells. These results are in agreement with the required CIITA co-localization with the PML bodies in the nucleus for the increased protein stability ${ }^{82,198}$, since only SUMOylated PML proteins are capable of orchestrating PML bodies ${ }^{180,181}$. Even though Ulbricht et al. have not evaluated the PML SUMOylation requirement for the increase in CIITA stability, our results concur with their published data showing that the PML proteins knockdown, and thus their SUMOylated forms, results in a drastic increase in CIITA degradation. More importantly, considering that SUMO is inducible by IFN- $\gamma^{197}$, the reported strong enhancement of PML II and CIITA interaction in the PML bodies induced by IFN- $\gamma$ treatment may suggest that the up-regulated SUMO-PML II interacts more efficiently with CIITA than the unmodified PML II protein.

Altogether, these data indicate that, at the basal levels of PML proteins, the strongest interaction promoted by the mono-SUMOylation of PML proteins is required for CIITA recruitment to the PML bodies and its protection from proteasomal degradation. Considering that PML bodies are potential hot spots for PTMs such as 
phosphorylation and acetylation $^{199}$, which were previously shown to be involved in the modulation of the proteasome recruitment for CIITA degradation ${ }^{77,79-81}$, it will be important to investigate if the PML body-localized PTMs contribute to the CIITA stability. Alternatively, the PML bodies may physically block the $19 \mathrm{~S}$ regulatory particle from binding to the CIITA degron region, stabilizing the protein by impairing proteasomal recruitment.

Based on our own data and the evidence available in the literature ${ }^{82,198}$, we propose a mechanistic model for the post-translational regulation of CIITA activity. We suggest that the non-immune cells can fine-tune the MHC II synthesis after induction of CIITA expression by regulating the levels of mono-SUMOylated PML proteins. The higher the SUMO-modified PML levels the stronger the protein-protein interaction between CIITA and PML proteins with consequent CIITA recruitment to the PML bodies in the nucleus, where the CIITA is protected from proteasomal degradation and remains transcriptionally active. In contrast, the downregulation of SUMO-modified PML proteins impairs the CIITA recruitment to the PML bodies, thereby maintaining CIITA susceptibility to proteasomal degradation and hampering the MHC II transactivation (Figure 32). It will be important to reveal the mechanism of differential regulation of mono-SUMOylated PML levels in non-immune cells. 


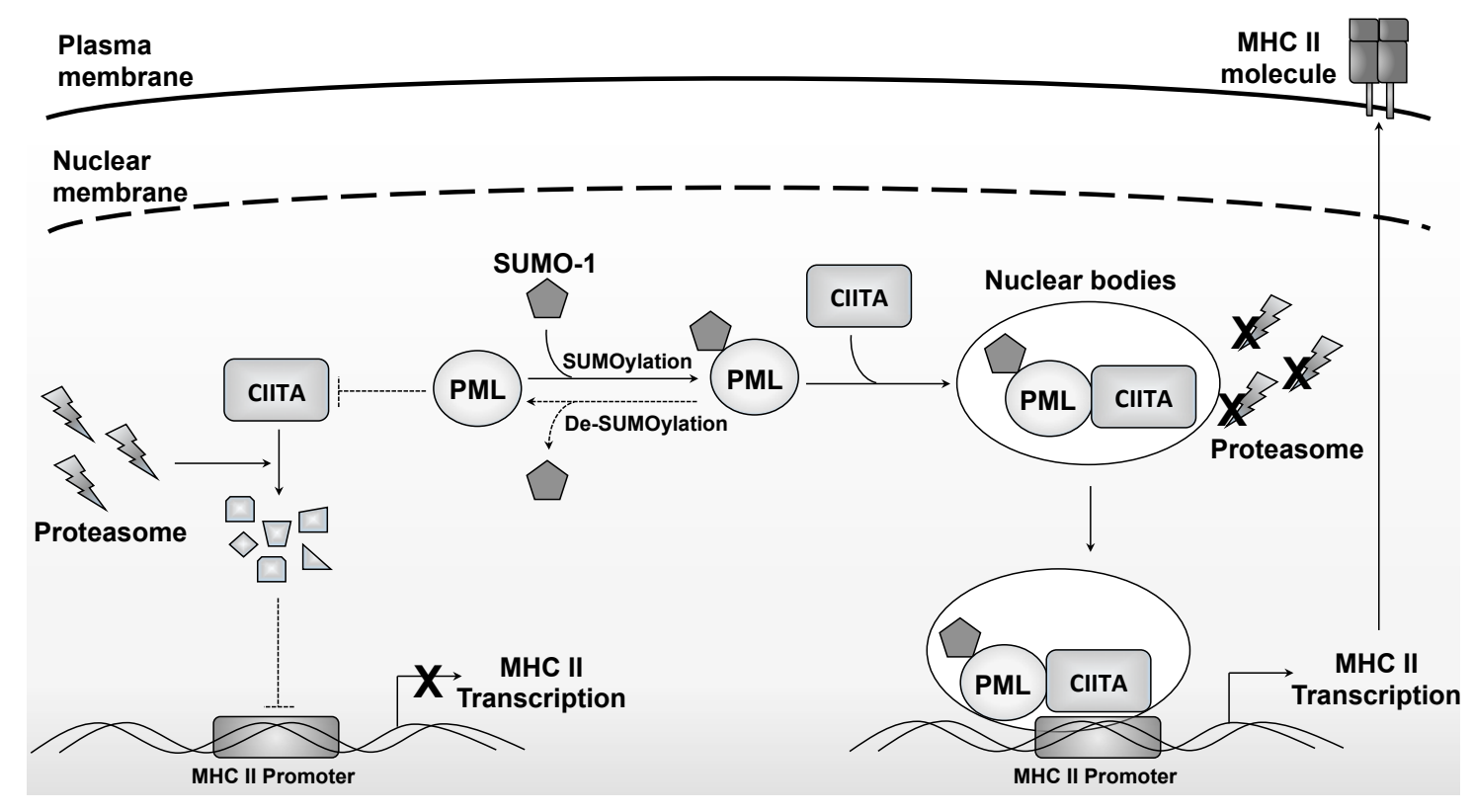

Figure 32 - A proposed mechanistic model for the post-translational regulation of CIITA in non-immune cells. CIITA is an unstable protein due to rapid proteasomal degradation. Nonimmune cells can increase the CIITA stability by inducing the PML proteins SUMOylation. Mono-SUMOylated PML proteins efficiently interact with CIITA, mediating its recruitment to the PML nuclear bodies, where the Class II Transactivator is protected from proteasome degradation by unkown mechanisms. The stabilized CIITA remains transcriptionally active for a prolonged period of time and drives the efficient expression of MHC II molecules. The induction of PML proteins de-SUMOylation impairs the PML-CIITA interaction in nonimmune cells, thus favoring the CIITA proteosomal degradation and ultimately blocking the MHC II expression. It is unclear if the CIITA-PML interaction occurs in the cytoplasm or nucleus.

Intriguingly, Huh-7 cells presented a particular behavior concerning the control of CIITA protein levels. Although the up-regulation of the mono-SUMOylated PML provided an increase in the CIITA levels, the regulation of modified PML did not greatly impact the CIITA kinetics resulting from the constant proteasome degradation of the newly synthesized CIITA proteins. Consequently, in this cell line the CIITA and MHC II expression were kept very low compared to the other cells. This special behavior may reflect the hepatocytes' need to tightly regulate CIITA activity in the absence of inflammatory conditions in order to avoid autoimmune liver diseases ${ }^{200,201}$ or fulminant hepatitis ${ }^{202}$, since this cell type presents the unusual ability to express costimulatory molecules ${ }^{203}$ and thus can activate $\mathrm{CD}^{+} \mathrm{T}$ cells more efficiently. 
Alternatively, as a hepatocarcinoma derived cell line, Huh-7 may present a tumorinduced defect specifically affecting the CIITA stability in order to impair the MHC II expression and evade immune detection ${ }^{97}$. Therefore, it will be important to validate our findings in primary human cells.

The regulation of CIITA proteasomal degradation described herein highlights the strict control of CIITA in non-immune cells. The expression of this factor is pivotal to the immune homeostasis, and if aberrantly activated can contribute to inflammationrelated diseases ${ }^{161,204,205}$. Previous studies have already described a number of different signaling pathways that fine-tune the IFN- $\gamma$-induced CIITA transcription. For instance, toll-like receptor 2 (TLR2) dependent mitogen-activated protein kinase (MAPK) signaling evokes histone hypoacetylation on the CIITA promoter region, dampening the transcription induced by IFN- $\gamma^{206}$. Several anti-inflammatory cytokines, including TGF$\beta$, IL-4 and IL-10, can all antagonize the IFN- $\gamma$ induced CIITA expression although the mechanisms vary ${ }^{207}$. Our findings add a post-translational mechanism to this complex regulation, indicating that even when subjected to the same stimuli combination, each tissue would be able to intrinsically control the CIITA stability and consequently modulate the capacity to stimulate $\mathrm{CD}^{+} \mathrm{T}$ cells through the MHC II antigen presentation, as an additional level of immune response modulation.

Furthermore, CIITA not only activates MHC II genes expression, but is also reported to regulate at least 43 genes $^{48}$ and bind to 480 loci only in B cells ${ }^{34}$. Since many of these newly identified genes do not contain the required promoter module for direct CIITA transactivation, it has been proposed that this protein can also indirectly activate genes by transactivation factors sequestering and chromatin remodeling ${ }^{48}$, or even by directly activating genes as a general transcription factor and functional homolog of the transcriptional initiation factor $\mathrm{TAF} 1^{47}$. More recently, this factor has 
also been implicated in novel tissue-specific roles, such as regulation of osteoclast differentiation and bone homeostasis ${ }^{208}$. In the light of this multiplicity of functions, the need for the additional regulatory mechanism described in the present study is evident for maintaining a tissue-specific CIITA activity.

Regardless of the post-translational regulation of CIITA, it is important to highlight that our sequence design strategy allowed the expression of a transcriptionally active protein in all non-immune cell lines transfected with the plasmid-based delivery system. Therefore, we considered the p/CIITA construct a suitable tool for further investigations of CIITA as a molecular adjuvant for the improvement of genetic immunization systems, such as DNA vaccinations.

The success of DNA vaccines relies on the efficient presentation of a DNA encoded antigen by different cells surrounding the vaccine administration area. Most of these cells are not professional APCs and only present the endogenously produced antigen through MHC I molecules, reducing vaccine potency. By co-expressing CIITA, all in vivo transfected cells would undergo MHC II mediated antigen presentation to $\mathrm{CD}^{+} \mathrm{T}$ cells, albeit with varying efficiencies, and the DNA vaccine would induce a broader and stronger immune response. In fact, the effectiveness of this adjuvant strategy for DNA vaccinations has already been shown by a single study that performed mice immunizations with a combination of tumor antigen DNA and CIITA DNA, resulting in enhanced antigen-specific $\mathrm{CD} 4^{+}$and $\mathrm{CD}^{+} \mathrm{T}$ cell responses, with the establishment of long-term protective memory against tumors ${ }^{209}$.

Although successful, this strategy used a DNA plasmid carrying the wild-type human CIITA cDNA containing the 5'and 3' UTRs ${ }^{209}$. Therefore, protein expression in that study may have been restricted by the aforementioned post-transcriptional regulatory mechanisms. Our expectation is that the use of the CIITA optimized 
sequence would potentiate the demonstrated adjuvant activity ${ }^{209}$. This hypothesis will be tested in future experimental studies involving the co-immunization of mice with $\mathrm{p} / \mathrm{CIITA}$ and a DNA plasmid encoding Dengue virus antigens, constituting the first study applying CIITA as molecular adjuvant for vaccines against an infectious disease.

Kim et al. ${ }^{209}$ also demonstrated that a mouse cell line of dendritic cells transfected with human CIITA DNA presented a higher expression of MHC II molecules and consequently an enhanced capacity to activate antigen-specific $\mathrm{CD}^{+} \mathrm{T}$ cells when compared to non-transfected DCs. Considering our research group's expertise in obtaining human dendritic cells through the in vitro differentiation from blood monocytes ${ }^{210-213}$, we reasoned to transfect DCs with the optimized CIITA sequence as a strategy to validate the factor expression and activity in a human primary cell model. In addition, given that DCs represent highly attractive targets for molecular immunotherapy of acquired diseases, such as AIDS and cancer, the efficient transfection of these cells with the designed sequence would also enable the future evaluation of CIITA as a molecular adjuvant for DC-based immunotherapeutic strategy.

To date, no efficient DNA transfection of monocyte-derived dendritic cells have been reported. Various conventional methods, including liposome complexes, electroporation, nucleoporation and $\mathrm{CaPO}_{4}$ precipitation, as well as some innovative methods, such as cationic peptide complexes and immunolipoplexes, were already tested, all resulting in inefficient gene transfer, low level of expression or low cell viability $^{214-223}$. In contrast, several studies have demonstrated efficient gene transfer to DCs with mRNA electroporation ${ }^{155,216,224-228}$ or viral vectors transduction ${ }^{186,229-236}$. Therefore, we attempted to develop both reported successful techniques to substitute the p/CIITA transfection in order to achieve efficient CIITA sequence transfer to mo-DCs. 
Initially, we developed an mRNA electroporation strategy, considering its simplicity over viral transduction protocols, and clinical safety profile (due to a strictly transient expression and inability to integrate into the host genome) ${ }^{237,238}$. Importantly, iDCs efficiently transfected with mRNA were shown to maintain the immature phenotype and the maturation potential after electroporation ${ }^{224,239}$. Taking these advantages into account, we planned to produce a bicistronic mRNA encoding the optimized CIITA sequence and the LNGFR surface marker, which would allow the identification and selection of CIITA-positive DCs after electroporation. However, the application of this strategy was compromised by the unexpected inability to produce the bicistronic mRNA in vitro.

Generally, the production of mRNA using commercial kits is a straightforward process that results in high yields of full-length capped mRNAs, as occurred for the production of control mRNAs codifying eGFP or LNGFR. In this process, a critical step is the preparation of proper templates through the linearization of DNA plasmids with a restriction enzyme downstream of the sequence to be transcribed. Circular plasmid templates generate extremely long, heterogeneous RNA transcripts, because RNA polymerases are highly processive enzymes. In the case of the successfully synthesized control mRNAs, the linear templates were easily produced by the digestion of pMACseGFP and pMACS plasmids, but the pMACS-CIITA construct was refractory to digestions using several different enzymes, impairing the in vitro transcription of the CIITA mRNA. The difficulty to digest the backbone of pMACS plasmid only when the CIITA sequence is present, as well as the impossibility to perform PCR amplification using primers specific to regions in the plasmid backbone, strongly suggests that the long CIITA optimized sequence insertion into pMACS is inducing DNA folding into 
highly condensed states ${ }^{240,241}$ that can impair the restriction enzymes ${ }^{242}$ and primers $\operatorname{access}^{243,244}$ to their specific sites.

While endeavoring to linearize pMACS-CIITA, the optimization of DCs electroporation was initiated using the eGFP mRNA. According to Chung et al. ${ }^{239}$ and Van Tendeloo et al. $^{224}$, the mRNA electroporation is more efficient when performed with iDCs rather than with mDCs. Based on this information, iDCs were utilized to first select the best voltage for mRNA delivery while not altering the other parameters, according to a previously described protocol ${ }^{155}$. Although Kavanagh et al. ${ }^{155}$ had reported a $75 \%$ transfection efficiency, our first test resulted in less than $2 \%$ of eGFPexpressing cells, even when using the same voltage applied in their work (400V). The method's toxicity was not mentioned by Kavanagh et al. ${ }^{155}$, which is curious since the electroporation we performed resulted in very low viability. The reason for such a discrepancy between our preliminary results and those previously published ${ }^{155}$ is unclear, and the comparison between different protocols toxicities is hampered by the meager information provided with respect to cell viability ${ }^{245,246}$. Furthermore, Van Tendeloo et al. ${ }^{224}$ suggested that cell toxicity is not solely due to the electroporation procedure itself, instead toxicity can also be related to the nature of the introduced nucleic acid. Even though a further protocol optimization could enhance the transfection efficiency and cell viability, difficulties in producing the CIITA mRNA, together with the aforementioned extremely low viability and transfection efficiency, were determining factors for the replacement of mRNA electroporation by the viral vector transduction of DCs.

Among the available viral vectors, both adenoviruses ${ }^{232-234,247}$ and lentiviruses ${ }^{186,231,235,236,248-251}$ have been used to successfully transduce mo-DCs, with adenoviral vectors being somewhat more efficient ${ }^{252}$. Nevertheless, the adenovirus- 
mediated transduction requires high viral doses and is accompanied by the coexpression of viral proteins that can be presented by transduced DCs and induce vectorspecific immunity ${ }^{247,253,254}$. On the other hand, lentiviruses do not express viral proteins and lower viral doses are sufficient for DCs transduction ${ }^{255}$. A comparative study also demonstrated that the adenoviral transduction induced more pronounced alterations in iDCs phenotype and function than the lentiviral transduction ${ }^{252}$. Moreover, lentiviruses pseudotyped with VSV-G envelope protein have been reported to transduce DCs without impairing their biological activity ${ }^{186,251,256,257}$. In light of the foregoing information, the VSV-G pseudotyped lentiviral vector was the preferred candidate for the establishment of DCs transduction.

At first, we planned on producing viral particles using the SiEW plasmid encoding the CIITA sequence, a lentiviral transfer vector previously used for DCs transduction strategies ${ }^{158}$. However, the generation of SiEW-CIITA was impaired due to the presence of sequences in the plasmid not stated in the vector map, as previously detected $^{258}$, which compromised the identification of the construct containing the properly oriented CIITA insert. Successful production of lentiviral particles was then achieved by using the LeGO-iG2 transfer vector.

Similar to SiEW, LeGO-iG2 presents an IRES-eGFP sequence and belongs to the third-generation of lentiviral vectors, which do not encode any viral proteins but contain cis-active elements for packing, reverse transcription and integration ${ }^{259}$. These vectors are replication defective, since the viral genes essential for virus production, e.g. gag and pol genes, are expressed in trans by a packing construct. Also, LeGO-iG2 is a self-inactivating vector in which the viral enhancer and promoter sequences within the 3' LTR have been deleted. This deletion prevents the possibility of insertional activation of cellular oncogenes by random integration of the vector into the genome reducing the 
risk of gene silencing in integrated provirus ${ }^{260,261}$. Therefore, the viral particles produced with LeGO-iG2 vector present high levels of biosafety and allow the identification of efficiently transduced cells by the detection of eGFP expression.

Both produced lentiviruses, LV-CIITA and LV-control, were able to transduce different types of human cell lines, as indicated by the eGFP expression. Also, the detection of MHC II molecules in cells transduced with LV-CIITA revealed the efficient induction of a functional CIITA protein expression by this lentivirus. Preliminary data showed the efficient CIITA expression, with nuclear localization, in primary human cells from skin explants inoculated with LV-CIITA. More importantly, after several adjustments in the transduction method, we finally succeeded in delivering the designed CIITA sequence into human DCs, as attested by the high levels of CIITA mRNA detected in the iDCs analyzed 7 days after LV-CIITA transduction. Therefore, our efforts in developing the lentiviral transduction system resulted in the establishment of a versatile tool that enables the CIITA sequence delivery to a broad range of cell types, including cells considered hard to transfect such as human DCs.

Curiously, unlike the transduction of human cell lines, the flow cytometric analysis of transduced iDCs was unable to detect significant eGFP expression, even though the alterations in cell morphology and the CIITA mRNA levels revealed efficient cell transduction. These discordant results might reflect the interference in the eGFP detection resulting from the cells fixation/permeabilization for the concurrent analysis of surface and intracellular MHC II molecules via flow cytometry ${ }^{262-264}$. Indeed, the fixation step was already associated with conformational changes that decrease eGFP brightness ${ }^{264}$ while the permeabilization causes the leach of this cytoplasmic molecule out of the cell ${ }^{262,263}$. In addition, Arthur et al. ${ }^{222}$ showed that even when the optimal transduction conditions are achieved, the levels of gene expression in 
DCs are generally significantly lower than those obtained using human cell lines. Considering these data from literature, it is possible that the high eGFP expression in transduced cell lines counteracted the deleterious effects of the fixation/permeabilization step, allowing the detection of eGFP-positive cells. Conversely, the loss of eGFP brightness and quantity during the intracellular staining associated with the low expression level in iDCs impaired the identification of efficiently transduced dendritic cells through the detection of eGFP fluorescence by flow cytometry.

Despite the inability to distinguish transduced from non-transduced cells by eGFP detection, we analyzed the surface and intracellular MHC II molecules expression in the entire DC population as a way to indirectly assess the CIITA expression and activity in transduced cells. For this analysis, the DCs were maintained in an immature stage, since the mature cells already express high levels of MHC II that would probably hinder the identification of the lentiviral-mediated increased expression. Using this approach we were unable to detect a CIITA-mediated enhancement of MHC II levels when comparing LV-CIITA and LV-control transductants, even though the CIITA mRNA detection up to 7 days post-transduction suggests that the protein might be produced by LV-CIITA-transduced iDCs.

Thus far only one study has performed the exogenous CIITA expression in human monocyte-derived DCs, which utilized the same flow cytometric analysis implemented here to demonstrate a significant increase in the MHC II expression by transfectants when compared to the mock control ${ }^{265}$. However, this increase was reported as percentage of MHC II-positive cells, with around $87 \%$ of CIITA-transfected cells and $50 \%$ of mock-transfected cells expressing this molecule. Since the study does not present the phenotypic analysis of in vitro generated DCs, and considering that the 
MHC II expression is a consistent feature of these cells, even at the immature stage, the reported results raise the question if the cells analyzed after transfection assays are in fact only dendritic cells or a mixture with contaminant cells such as undifferentiated monocytes. The expected results from flow cytometry analysis restricted to DCs would present $100 \%$ of MHC II-positive cells, with the CIITA overexpression inducing an increase in the protein production per cell, which could be visualized by an augmented MFI. Therefore, we cannot ensure that comparisons between our results and the mentioned work are worthwhile.

The lack of CIITA-specific induction of MHC II molecules described here may reflect the strict control of the factor expression by immature dendritic cells. Although these cells present an active transcription of the endogenous CIITA gene and are known to express the protein, the CIITA is maintained at levels undetectable by our western blot technique, indicating the existence of post-transcriptional mechanisms restricting the protein expression. Accordingly, western blot assays revealed a downregulation in the levels of mono-SUMOylated PMLs during the monocytes differentiation into iDCs, reaching levels approximately 60\% lower than the levels found in HEK293 cells. Given that the regulation of SUMO-modified PML proteins were associated with the CIITA protection from proteasomal degradation, it is possible that iDCs maintain low levels of CIITA protein due to increased proteasomal degradation resultant from the downregulation of mono-SUMOylated PMLs. Thus, even in the presence of high levels of CIITA mRNA, the efficient exogenous CIITA expression would be impaired in LVCIITA-transduced iDCs through post-translational mechanisms, resulting in the unexpected lack of MHC II transactivation. The direct investigation of CIITA protein levels in transduced iDCs by western blot and immunofluorescence assays will easily confirm or refute this hypothesis. 
Alternatively, our inability to detect the CIITA-specific induction of MHC II molecules may reflect limitations of our transduction system, not the inefficient CIITA expression in iDCs. In this regard, the comparative analysis between transduced and non-transduced iDCs revealed the unspecific induction of MHC II expression mediated by viral vectors, since both LV-CIITA and LV-control transductants presented a 2- to 3fold increase in the molecules expression when compared to non-transduced iDCs. This strong maturation stimulus provided by the viral vectors may have exhausted the cells capacity to de novo synthesize MHC II molecules, thus impeding further protein expression mediated by the exogenous CIITA.

In agreement with the unspecific maturation stimuli provided by the lentiviruses, the expression of other surface molecules that are not regulated by CIITA factor, such as CD80, CD83 and CD40, was also found to be elevated in iDCs transduced with both viral vectors, and the highest protein levels were associated with LV-control transduction. In fact, lentiviruses can trigger the maturation of iDCs by a number of pathways that results in nuclear factor- $\kappa \mathrm{B}(\mathrm{NF}-\kappa \mathrm{B})$ activation $^{266}$. Tan et al. ${ }^{252}$ demonstrated that the main pathway involved is that mediated by protein kinase $\mathrm{R}$ $(\mathrm{PKR})^{267}$. Constitutively expressed PKR is activated through autophosphorylation initiated by the direct binding of double stranded RNA (dsRNA), an intermediate stage in the lentiviral life cycle. The phospho-PKR (p-PKR) phosphorylates the inhibitor of kappa $\mathrm{B}(\mathrm{I \kappa B})$, which in turn results in the activation of nuclear NF-kB and thus the transcription of several genes with anti-viral activity. Breckpot et al. ${ }^{185}$ also demonstrated that during lentiviral transduction, the activation of several TLRs results in NF- $\mathrm{BB}$ activation and consequent iDC maturation. In that study, high doses of lentiviral vectors (MOI of 15 or higher) resulted in the activation of TLR2, TLR3 and 
TLR8, which are engaged by virion components, dsRNA, and single strand RNA (ssRNA), respectively.

Although both aforementioned studies have correlated iDC maturation only with high doses of lentiviral vectors, and previous works had contrarily reported the maintenance of an immature phenotype after transduction ${ }^{186,255,256}$, the parallel comparison of the transduction effects on DCs phenotype is unreliable due to variability among studies. First, different lentiviral systems were used; second, DCs differentiation was induced with different concentration of cytokines according to distinct culture conditions; third, virus titers were determined in different cell lines; and forth, the transduction method varied. At least in our hands, even at low viral dose (MOI of 2), the viral vectors induced iDC maturation, and the strongest induction by LV-control indicates its higher DC transduction efficiency compared to LV-CIITA, resulting in a higher concentration of viral components per transduced cell and consequently, augmented activation of NF- $\mathrm{KB}$.

Considering our hypothesis that this induced maturation has reached the maximum capacity of iDCs to produce MHC II molecules, the CIITA delivery system developed here compromises the detection of MHC II transactivation activity. If this is the case, the transcriptionally active CIITA protein may be efficiently expressed by transduced iDCs, and can still interfere with the cell capacity to activate the $\mathrm{T}$ cell immune response through the regulation of other target genes such as plexin-A1, IL-10 and IL-4. This hypothesis can be easily tested through the co-cultivation of transduced DCs with autologous $\mathrm{T}$ cells followed by the analysis of $\mathrm{T}$ cell proliferation and activation.

Although time and samples restrictions hampered the functional analysis of transduced iDCs, their capacity to stimulate an antigen-specific $\mathrm{T}$ cell response can be 
performed using samples derived from HIV positive donors, since iDCs from these individuals were also efficiently transduced with LV-CIITA and presented a similar unspecific induction of maturation. Through the co-cultivation of LV-control- and LVCIITA-transduced iDCs with autologous $\mathrm{T}$ cells from these donors, we can easily evaluate the activation of antigen-specific response through the pulse of iDCs with HIV-1 peptides or even the inactivated autologous virus, and therefore indirectly determine if the CIITA protein is expressed in the transduced cells.

In the chance that the CIITA protein expression and activity is confirmed by the aforementioned assays, our perspective is to further investigate if the CIITA overexpression in DCs from HIV+ individuals would improve the immunotherapy potency. The use of CIITA as a molecular adjuvant for dendritic cell-based immunotherapies against HIV is particularly interesting considering that some studies have demonstrated that, despite presenting a functional maturation phenotype, DCs exposed to gp120 or AT-2 inactivated virus ${ }^{150,151}$ can induce an immunossupressive environment. This immunossupression was directly correlated with an enhanced production of IL-10 by $\mathrm{mDCs}$, mediated by ERK1/2 signaling pathway triggered by gp120 interaction with mannose C-type lectin receptors (MCLRs) ${ }^{151}$. Expression of IL10 by DCs can direct the polarization of naïve $\mathrm{T}$ cells to Tregs, as well as inhibit $\mathrm{T}$ cell proliferation and cytokine production ${ }^{52}$, such as IL-12 $2^{150,268}$. Hence, the CIITA overexpression in DCs may circumvent the HIV-induced immunossupression by inhibiting the IL-10 transcription $^{51}$. Furthermore, CIITA overexpression may also improve immunotherapy potency by upregulating plexin- $\mathrm{A} 1^{50}$ and promoting more efficient immunologic synapses between DC and T cells, not considering the regulation of unknown genes that may also influence the DC functionality. 
Even though the lentiviral transduction established here is not the ideal system for the optimized CIITA delivery to DCs due to the unspecific induction of cell maturation, the use of this strategy will still enable the evaluation of the combined effect of non-MHC II genes controlled by CIITA in the immune activation capacity of DCs. In addition, the protocols to mature dendritic cells into three mDC subtypes (DC-0, DC-1 and DC-2) established in the present study will allow the comparison of LV-CIITA transduced iDCs subjected to the different maturation cocktails in order to evaluate which subtype induces a more potent and effective $\mathrm{T}$ cell response against HIV when overexpressing CIITA. Although mouse models have already demonstrated that CIITA does not interfere in IL-12 production by DCs ${ }^{51}$, this factor may direct DC function in alternative and still unknown mechanisms. Therefore, the comparison of transduced mDC subtypes may also reveal whether or not the overexpression of this factor would alter the type of immune response induced by each polarizing DC.

To summarize, we reported the development of two delivery systems that enable the human CIITA factor expression and transcriptional activity in different nonimmune human cell types and the efficient transduction of the hard to transfect human dendritic cells. The future application of these tools in in vitro studies, involving DNA vaccinations and dendritic cell-based immunotherapies, will allow the evaluation of CIITA as a molecular adjuvant and contribute to the better understanding of the critical role this factor plays in the initiation of the adaptive immune response. Importantly, the present work brought to light a new and intricate mechanism of CIITA posttranslational regulation involving the modulation of proteasomal-mediated degradation, thus contributing to the understanding of the complex regulatory layers of CIITA activity. 


\section{CONCLUSIONS}


- The p/CIITA DNA construct carrying the optimized human CIITA sequence successfully induces the expression of a transcriptionally active factor, with proper intracellular distribution, in different types of non-immune cells;

- The LV-CIITA lentivirus carrying the same optimized sequence also induces the expression of a transcriptionally active CIITA protein in different types of nonimmune cells;

- Both CIITA delivery systems established successfully induce the de novo expression of MHC II molecules in all non-immune human cell types evaluated;

- The p/CIITA and LV-CIITA are valid gene delivery systems for the investigation of the potential CIITA adjuvant activity for vaccines and immunotherapies;

- Non-immune cells differentially modulate CIITA activity by an additional regulatory mechanism that interferes with the proteasomal degradation of CIITA;

- Each non-immune cell type can differentially controls the CIITA degradation by modulating the recruitment of this factor to the PML nuclear bodies, which impairs the proteasomal degradation by unknown mechanisms;

- CIITA recruitment to PML bodies is dependent on the interaction with the mono-SUMOylated PML proteins, and each cell type differentially regulates the levels of these SUMO-modified proteins as a means to regulate CIITA proteasomal degradation;

- Monocyte-derived dendritic cells express very low levels of endogenous CIITA at the immature stage, and the monocyte differentiation into iDCs is accompanied by a reduction in the levels of mono-SUMOylated PML proteins, 
suggesting that iDCs may also regulate CIITA activity by modulating its proteasomal degradation;

- The LV-CIITA vector efficiently transduce the primary human mo-DCs and induce a sustained CIITA mRNA synthesis in iDCs from healthy individuals and chronically infected HIV-1 patients;

- The lentiviral transfer vectors by themselves induce an unspecific phenotypic maturation of transduced iDCs of healthy and HIV-1-infected individuals, which impairs the indirect evaluation of exogenous CIITA expression through the analysis of its MHC II transactivation activity. 
7 APPENDIX 


\section{A - Optimization Report}

antelechon

\section{Optimization report}

Created with Leto 1.0

\section{General Information:}

$$
\begin{gathered}
\text { Gene: CIITA_2A } \\
\text { Optimization requested by: } \\
\text { Date: Aug 26, } 2011 \\
\text { Operator: rdhalia }
\end{gathered}
$$

Target organism: Homo sapiens Cut_off 50\% Optimization iteration: 1

Length: $3498 \mathrm{bp}$

\subsection{Sequence}

Below the original sequence and optimized sequence are listed, labelled with 'Org:' and 'Opt:' respectively. Where both are identical, the original sequence contains dots instead of nucleotides ('.'). Where the original sequence was locked against editing, it contains underscores ('_'). The names of the restriction enzymes start at the first nucleotide after the restriction cut in the forward strand.

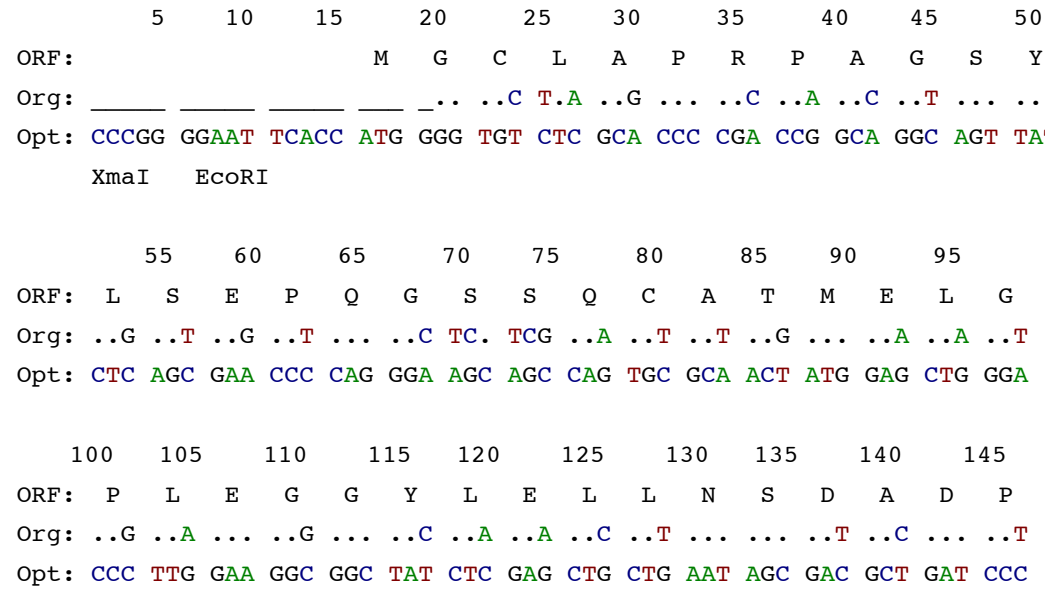




\begin{tabular}{|c|c|c|c|c|c|c|c|c|c|c|c|c|c|c|c|}
\hline & 150 & & 155 & & 160 & 165 & & 170 & & 175 & 180 & & 185 & & 90 \\
\hline RF : & L & C & L & $\mathrm{Y}$ & $\mathrm{H}$ & F & $\mathrm{Y}$ & D & $Q$ & M & D & L & A & G & E \\
\hline q: & $\cdots A$ & $\cdots$ & . T & $\cdots$ & $\cdots$ & $\cdots \mathrm{T}$ & $\ldots \mathrm{T}$ & ..T & $\cdots A$ & $\cdots$ & $\cdots$ & T.A & $\ldots A$ & $\cdots A$ & $\cdots$ \\
\hline
\end{tabular}

$\begin{array}{lllllllll}200 & 205 & 210 & 215 & 220 & 225 & 230 & 235 & 240\end{array}$

$\begin{array}{llllllllllllllllll}\text { ORF : } & \text { E } & \text { I } & \text { E } & \text { L } & \text { Y } & \text { S } & \text { E } & \text { P } & \text { D } & \text { T } & \text { D } & \text { T } & \text { I } & \text { N } & C & \text { D }\end{array}$

Org: ..А $\ldots$.

Opt: GAG ATC GAG CTG TAT AGC GAA CCG GAT ACC GAT ACC ATC AAC TGT GAC

$\begin{array}{cccccccccccccccccc} & 245 & & 250 & 255 & & 260 & & 265 & 270 & & 275 & 280 & 285 & & 290 \\ \text { ORF : } & Q & \text { F } & \text { S } & \text { R } & \text { L } & \text { L } & \text { C } & \text { D } & \text { M } & \text { E } & \text { G } & \text { D } & \text { E } & \text { E } & \text { T } & \text { R }\end{array}$

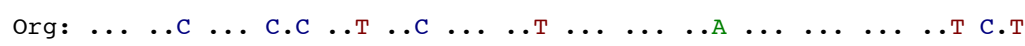
opt: CAg tTT AGC AGA CTG CTG TGC GAC ATG GAA GGT GAC GAA GAG ACA AGA

$\begin{array}{llllllllllllllllllll} & & & 295 & 300 & & 305 & & 310 & 315 & & 320 & 325 & 330 & & 335 & \\ \text { ORF : } & \text { E } & \text { A } & \text { Y } & \text { A } & \text { N } & \text { I } & \text { A } & \text { E } & \text { L } & \text { D } & \text { Q } & \text { Y } & \text { V } & \text { F } & \text { Q } & \text { D }\end{array}$

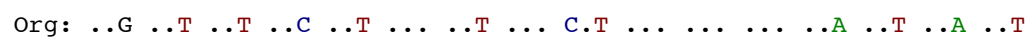
Opt: GAA GCC TAC GCT AAC ATC GCA GAG TTG GAC CAG TAC GTG TTC CAG GAC

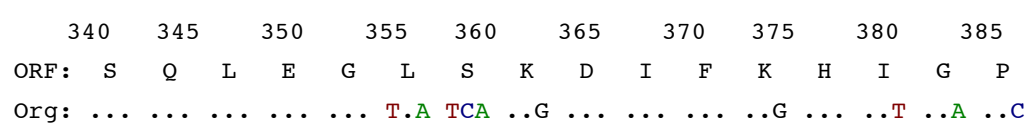
Opt: TCC CAA CTG GAG GGT CTT AGC AAA GAT ATC TTC AAA CAC ATC GGT CCT

$\begin{array}{ccccccccccccccccccc} & 390 & & 395 & & 400 & 405 & & 410 & & 415 & 420 & & 425 & & 430 & 435 \\ \text { ORF : } & \text { D } & \text { E } & \text { V } & \text { I } & \text { G } & \text { E } & \text { S } & \text { M } & \text { E } & \text { M } & \text { P } & \text { A } & \text { E } & \text { V } & \text { G } & \text { Q } \\ \text { Org : } & \ldots & \ldots & \ldots & \ldots & \ldots & \ldots & \ldots & \ldots & \ldots & \ldots & \ldots & \ldots & \ldots & \ldots & \ldots & \ldots & \ldots & \ldots\end{array}$
Opt: GAT GAG GTG ATC GGG GAG AGC ATG GAG ATG CCT GCC GAA GTG GGA CAG

$\begin{array}{lcccccccccccccccc} & & 440 & & 445 & 450 & & 455 & & 460 & 465 & & 470 & & 475 & 480 & \\ \text { ORF : } & \mathrm{K} & \mathrm{S} & \mathrm{Q} & \mathrm{K} & \mathrm{R} & \mathrm{P} & \mathrm{F} & \mathrm{P} & \mathrm{E} & \mathrm{E} & \mathrm{L} & \mathrm{P} & \mathrm{A} & \mathrm{D} & \mathrm{L} & \mathrm{K}\end{array}$

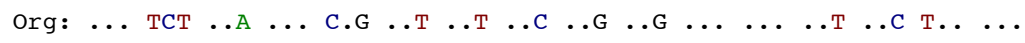
Opt: AAA AGC CAG AAG AGA CCC TTC CCG GAA GAA CTG CCA GCG GAT CTG AAA $\begin{array}{rcccccccccccccccc}485 & 490 & 495 & & 500 & 505 & 510 & & 515 & 520 & 525 & & 530 \\ \text { ORF : } & \text { H } & \text { W } & \text { K } & \text { P } & \text { A } & \text { E } & \text { P } & \text { P } & \text { T } & \text { V } & \text { V } & \text { T } & \text { G } & \text { S } & \text { L } & \text { L }\end{array}$

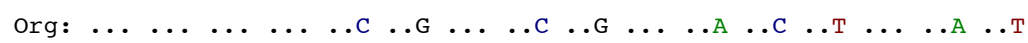
Opt: CAC TGG AAA CCA GCT GAA CCC CCA ACT GTT GTG ACT GGA AGC CTG CTG

\begin{tabular}{|c|c|c|c|c|c|c|c|c|c|c|c|c|c|c|c|}
\hline & & 35 & 540 & & 545 & & 50 & 555 & & 560 & & 65 & 570 & & 575 \\
\hline ORF : & V & G & P & $\mathrm{V}$ & $\mathrm{S}$ & D & C & $S$ & $\mathrm{~T}$ & L & P & C & L & P & L \\
\hline Org: & $\ldots G$ & $\ldots$ & $\ldots C$ & $\cdots$ & TCG & $\cdots$ & $\ldots T$ & $\ldots T$ & $\ldots A$ & С.T & $\ldots \mathrm{A}$ & . & $\ldots C$ & $\ldots G$ & $\ldots A$ \\
\hline Opt: & GTC & GGA & CCT & GTT & AGC & GAC & TGC & AGC & ACC & TTG & ССТ & TGC & СTT & $\mathrm{CCC}$ & СТC \\
\hline
\end{tabular}


$\begin{array}{rcccccccccccccr}580 & 585 & & 590 & & 595 & 600 & & 605 & & 610 & 615 & 620 & 625 \\ \text { A } & \text { L } & \text { F } & \text { N } & \text { Q } & \text { E } & \text { P } & \text { A } & \text { S } & \text { G } & \text { O } & \text { M } & & & \end{array}$

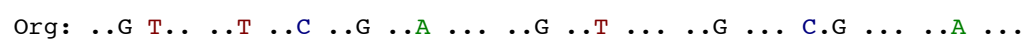
Opt: GCA CTG TTC AAT CAA GAG CCA GCC AGC GGA CAA ATG AGA CTG GAG AAG $\begin{array}{llllllllll}630 & 635 & 640 & 645 & 650 & 655 & 660 & 665 & 670 & 675\end{array}$

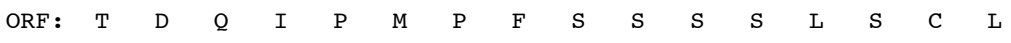

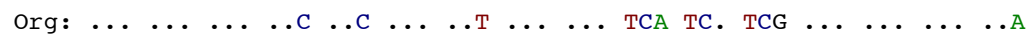
Opt: ACA GAC CAG ATA CCT ATG CCC TTT AGC AGC AGC AGT CTG TCT TGC CTG $\begin{array}{lcccccccccccccccc} & & 680 & & 685 & 690 & & 695 & & 700 & 705 & & 710 & & 715 & 720 \\ \text { ORF : } & \text { N } & \text { L } & \text { P } & \text { E } & \text { G } & \text { P } & \text { I } & \text { Q } & \text { F } & \text { V } & \text { P } & \text { T } & \text { I } & \text { S } & \text { T } & \text { L }\end{array}$

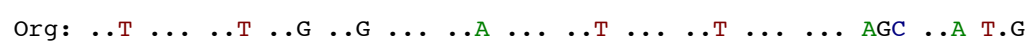
Opt: AAC CTC CCA GAA GGA CCT ATT CAG TTC GTG CCC ACC ATT TCA ACG CTC

$\begin{array}{ccccccccccccccccc} & 725 & & 730 & 735 & & 740 & & 745 & 750 & & 755 & & 760 & 765 & & 770 \\ \text { ORF : } & \text { P } & \text { H } & \text { G } & \text { L } & \text { W } & \text { Q } & \text { I } & \text { S } & \text { E } & \text { A } & \text { G } & \text { T } & \text { G } & \text { V } & \text { S } & \text { S }\end{array}$

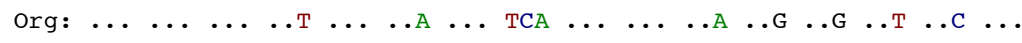
Opt: CCC CAC GGC CTG TGG CAg ATA AGC GAg GCT GGg ACA GGC GTG TCT AGC $\begin{array}{lccccccccccccccccc} & & 775 & 780 & & 785 & & 790 & 795 & & 800 & & 805 & 810 & & 815 & \\ \text { ORF : } & \text { I } & \text { F } & \text { I } & \text { Y } & \text { H } & \text { G } & \text { E } & \text { V } & \text { P } & \text { Q } & \text { A } & \text { S } & \text { Q } & \text { V } & \text { P } & \text { P }\end{array}$

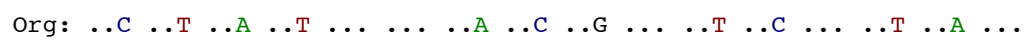
opt: ATA TTC ATC TAC CAC GGC GAg GTT CCC CAg GCG AGT CAg GTG CCG CCA

$\begin{array}{llllllllll}820 & 825 & 830 & 835 & 840 & 845 & 850 & 855 & 860 & 865\end{array}$

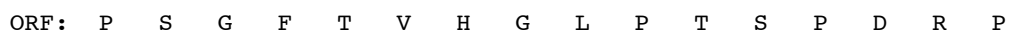

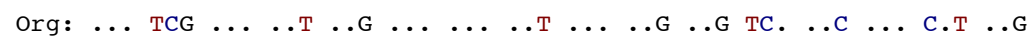
Opt: CCA AGC GGC TTC ACA GTG CAC GGA CTG CCC ACA AGT CCA GAC AGG CCA

$\begin{array}{llccccccccccccccc} & 870 & & 875 & & 880 & 885 & & 890 & & 895 & 900 & & 905 & & 910 & 915 \\ \text { ORF : } & \text { G } & \text { S } & \text { T } & \text { S } & \text { P } & \text { F } & \text { A } & \text { P } & \text { S } & \text { A } & \text { T } & \text { D } & \text { L } & \text { P } & \text { S } & \text { M }\end{array}$ Org: $\ldots$ ТСТ $\ldots$ Т $\ldots$. . $\ldots$. Opt: GGC AGC ACC TCC CCT TTC GCG CCT AGC GCT ACT GAC CTT CCC AGC ATG $\begin{array}{lcccccccccccccccc} & & 920 & & 925 & 930 & & 935 & & 940 & 945 & & 950 & & 955 & 960 & \\ \text { ORF : } & \text { P } & \text { E } & \text { P } & \text { A } & \text { L } & \text { T } & \text { S } & \text { R } & \text { A } & \text { N } & \text { M } & \text { T } & \text { E } & \text { H } & \text { K } & \text { T }\end{array}$

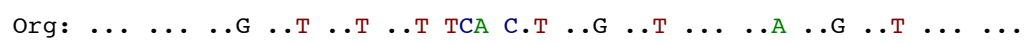
Opt: CCA GAG CCC GCC CTG ACC AGC AGg GCT AAC ATG ACC GAA CAC AAG ACG

$\begin{array}{llllllllll}965 & 970 & 975 & 980 & 985 & 990 & 995 & 1000 & 1005 & 1010\end{array}$

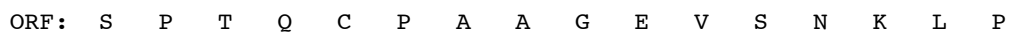

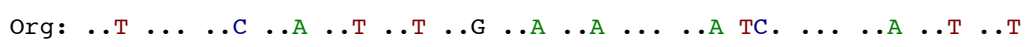
Opt: AGC CCC ACT CAG TGC CCC GCC GCC GGC GAA GTG AGC AAC AAG CTG CCC 
$\begin{array}{lcccccccccccccccc}1015 & 1020 & 1025 & 1030 & 1035 & 1040 & 1045 & 1050 & 1055 & \\ \text { ORF : } & \text { K } & \text { W } & \text { P } & \text { E } & \text { P } & \text { V } & \text { E } & \text { Q } & \text { F } & \text { Y } & \text { R } & \text { S } & \text { L } & \text { Q } & \text { D } & \text { T }\end{array}$

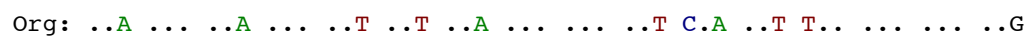
Opt: AAG TGG CCT GAA CCA GTG GAG CAG TTC TAC AGG AGC CTG CAG GAC ACT

$\begin{array}{llllllllll}1060 & 1065 & 1070 & 1075 & 1080 & 1085 & 1090 & 1095 & 1100 & 1105\end{array}$ $\begin{array}{llllllllllllllllll}\text { ORF : } & Y & G & A & E & P & A & G & P & D & G & I & L & V & E & V & D\end{array}$

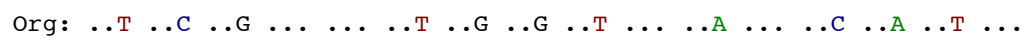
Opt: TAC GGA GCC GAG CCT GCA GGA CCT GAC GGT ATC CTG GTG GAG GTG GAC

$\begin{array}{llllllllll}1110 & 1115 & 1120 & 1125 & 1130 & 1135 & 1140 & 1145 & 1150 & 1155\end{array}$

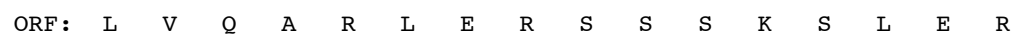

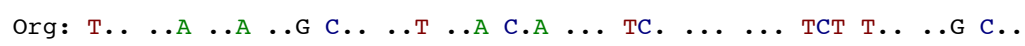
Opt: CTG GTG CAg GCT AgG CTG GAg AgG AGC AGC AGC AAA AGC CTG GAA AGA

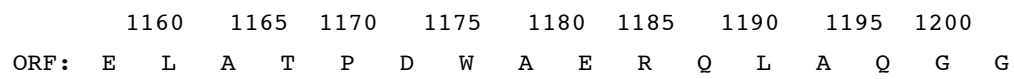

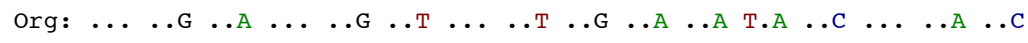
Opt: GAg CTT GCG ACA CCC GAC TGG GCA GAA AGg CAg CTG GCA CAg GGT GGA

$\begin{array}{llllllllll}1205 & 1210 & 1215 & 1220 & 1225 & 1230 & 1235 & 1240 & 1245 & 1250\end{array}$ $\begin{array}{lllllllllllllllllll}\text { ORF : } & L & A & E & V & L & L & A & A & K & E & H & R & R & P & R & E\end{array}$

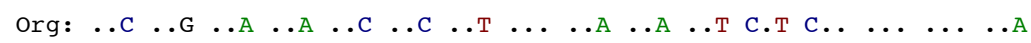
Opt: CTG GCT GAG GTC CTG CTG GCC GCT AAg GAG CAC AGA AGG CCC AGG GAG

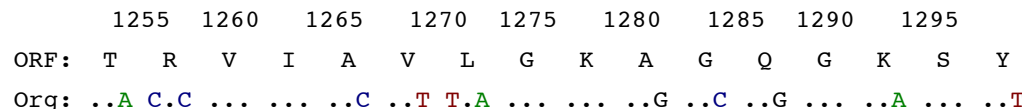
Opt: ACG AGg GTG ATC GCT GTG CTC GGA AAA GCA GGA CAA GGG AAG TCC TAC

$\begin{array}{llllllllll}1300 & 1305 & 1310 & 1315 & 1320 & 1325 & 1330 & 1335 & 1340 & 1345\end{array}$ $\begin{array}{llllllllllllllllll}\text { ORF : } & W & A & G & A & \text { V } & \text { S } & \text { R } & \text { A } & \text { W } & \text { A } & \text { C } & \text { G } & \text { R } & \text { L } & \text { P } & \text { Q }\end{array}$

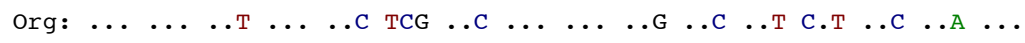
Opt: TGG GCT GGA GCT GTG AGC CGT GCC TGG GCT TGT GGC AGG CTG CCC CAG $\begin{array}{llllllllll}1350 & 1355 & 1360 & 1365 & 1370 & 1375 & 1380 & 1385 & 1390 & 1395\end{array}$ $\begin{array}{llllllllllllllllllll}\text { ORF : } & Y & D & F & V & F & S & V & P & C & H & C & L & N & R & P & G\end{array}$

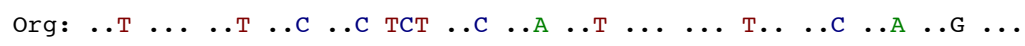
Opt: TAC GAT TTC GTG TTT AGC GTT CCG TGC CAC TGT CTG AAT AgG CCA GGA

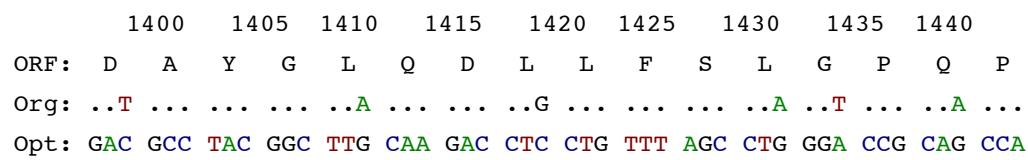


$\begin{array}{llllllllll}1445 & 1450 & 1455 & 1460 & 1465 & 1470 & 1475 & 1480 & 1485 & 1490\end{array}$ $\begin{array}{llllllllllllllllllllll}\text { ORF : } & \text { L } & \text { V } & \text { A } & \text { A } & \text { D } & \text { E } & \text { V } & \text { F } & \text { S } & \text { H } & \text { I } & \text { L } & K & R & P & D\end{array}$ Org: $\ldots$ A $\ldots$. $\ldots$. Opt: CTG GTG GCT GCC GAC GAG GTG TTC AGC CAC ATC CTG AAG AGA CCC GAT

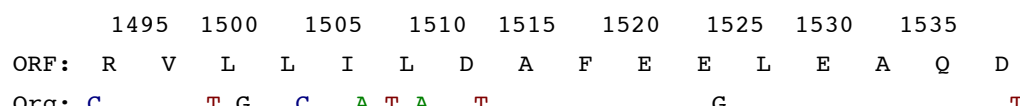

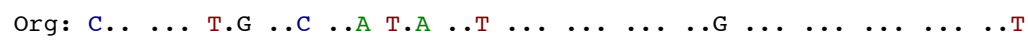
Opt: AGg GTG CTC CTG ATC CTG GAC GCC TTT GAG GAA CTG GAG GCA CAA GAC

$\begin{array}{llllllllll}1540 & 1545 & 1550 & 1555 & 1560 & 1565 & 1570 & 1575 & 1580 & 1585\end{array}$ $\begin{array}{llllllllllllllllll}\text { ORF : } & G & F & \text { L } & H & S & \text { T } & C & G & P & A & P & A & E & P & C & S\end{array}$

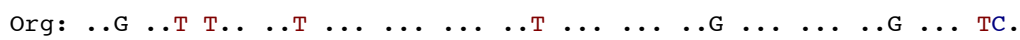
Opt: GGC TTC CTG CAC AGC ACC TGT GGG CCC GCT CCT GCA GAA CCC TGC AGT

$\begin{array}{llllllllll}1590 & 1595 & 1600 & 1605 & 1610 & 1615 & 1620 & 1625 & 1630 & 1635\end{array}$ $\begin{array}{llllllllllllllllll}\text { ORF : } & \text { L } & R & G & \text { L } & \text { L } & \text { A } & G & \text { L } & F & Q & K & K & \text { L } & \text { L } & R & G\end{array}$ Org: Т.. С.Т ..А $\ldots$ С Т...Т $\ldots$. Opt: CTG AGA GGC CTG CTG GCA GGA CTG TTC CAG AAg AAA CTC CTG CGA GGT

$\begin{array}{lcccccccccccccccc} & 1640 & 1645 & 1650 & 1655 & 1660 & 1665 & 1670 & 1675 & 1680 & \\ \text { ORF : } & \text { C } & \text { T } & \text { L } & \text { L } & \text { L } & \text { T } & \text { A } & \text { R } & \text { P } & \text { R } & \text { G } & \text { R } & \text { L } & \text { V } & \text { Q } & \text { S }\end{array}$

Org: ..С $\ldots$. . . . . . . . Opt: TGT ACC CTT CTT CTG ACC GCT CGA CCT CGA GGA AGA CTG GTC CAG TCA

$\begin{array}{llllllllll}1685 & 1690 & 1695 & 1700 & 1705 & 1710 & 1715 & 1720 & 1725 & 1730\end{array}$ $\begin{array}{lllllllllllllllllll}\text { ORF : } & L & S & K & A & D & A & \text { L } & F & E & \text { L } & S & G & F & S & M & E\end{array}$

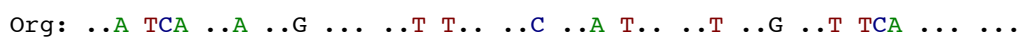
Opt: CTG AGC AAG GCA GAC GCC CTG TTT GAG CTG AGC GGC TTC AGC ATG GAG

$$
\begin{array}{lllllllll}
1735 & 1740 & 1745 & 1750 & 1755 & 1760 & 1765 & 1770 & 1775
\end{array}
$$

$\begin{array}{lllllllllllllllll}\text { ORF : } & Q & A & Q & A & Y & V & M & R & Y & F & E & S & S & G & M & T\end{array}$

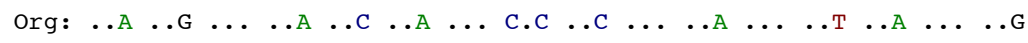
Opt: CAG GCA CAA GCC TAT GTG ATG AGG TAT TTC GAG AGC AGC GGG ATG ACT

$\begin{array}{llllllllll}1780 & 1785 & 1790 & 1795 & 1800 & 1805 & 1810 & 1815 & 1820 & 1825\end{array}$ $\begin{array}{llllllllllllllllllll}\text { ORF : } & E & H & Q & D & R & A & L & T & L & L & R & D & R & P & L & L\end{array}$

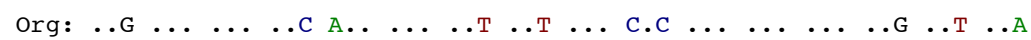
Opt: GAA CAC CAG GAT CGA GCC CTG ACC CTG TTG AGG GAT CGA CCA CTC CTC

$\begin{array}{llllllllll}1830 & 1835 & 1840 & 1845 & 1850 & 1855 & 1860 & 1865 & 1870 & 1875\end{array}$ $\begin{array}{lllllllllllllllllllllll}\text { ORF : } & L & S & H & S & H & S & P & T & L & C & R & A & V & C & Q & L\end{array}$

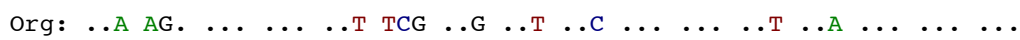
Opt: CTG TCT CAC TCC CAC AGT CCA ACA CTG TGC AGG GCC GTG TGT CAG CTG 


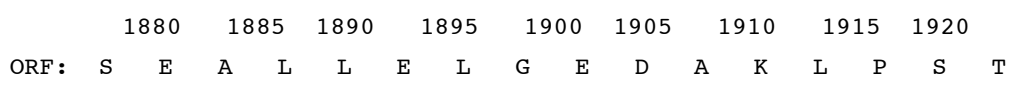

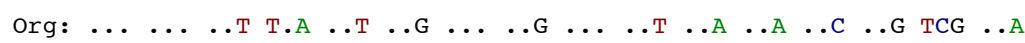
Opt: AGC GAA GCC CTC CTG GAA CTG GGC GAA GAC GCC AAG CTG CCC AGC ACC

$\begin{array}{llllllllll}1925 & 1930 & 1935 & 1940 & 1945 & 1950 & 1955 & 1960 & 1965 & 1970\end{array}$ ORF: $\begin{array}{llllllllllllllllll} & \text { T } & G & \text { L } & \text { Y } & \text { V } & \text { G } & \text { L } & \text { L } & \text { G } & \text { R } & \text { A } & \text { A } & \text { L } & \text { D } & S\end{array}$

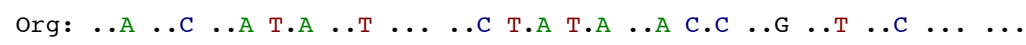
Opt: СTC ACT GGC CTG TAC GTG GGG CTG CTG GGC AGA GCT GCC CTG GAT AGC

$$
\begin{array}{lllllllll}
1975 & 1980 & 1985 & 1990 & 1995 & 2000 & 2005 & 2010 & 2015
\end{array}
$$

$\begin{array}{lllllllllllllllll}\text { ORF : } & P & P & G & A & \text { L } & \text { A } & \text { E } & \text { L } & \text { A } & \text { K } & \text { L } & \text { A } & \text { W } & \text { E } & \text { L } & G\end{array}$

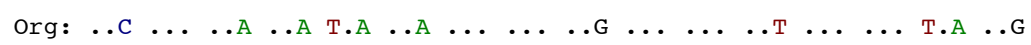
Opt: CCA CCT GGT GCC CTG GCG GAA CTG GCC AAg CTG GCA TGG GAG CTC GGC

$\begin{array}{llllllllll}2020 & 2025 & 2030 & 2035 & 2040 & 2045 & 2050 & 2055 & 2060 & 2065\end{array}$ $\begin{array}{llllllllllllllllll}\text { ORF : } & R & R & H & Q & S & T & L & Q & E & D & Q & F & P & S & A & D\end{array}$ Org: $\ldots$. . . Opt: AGg CGT CAC CAG TCA ACG CTG CAG GAA GAT CAA TTC CCG AGC GCA GAC

$\begin{array}{llllllllll}2070 & 2075 & 2080 & 2085 & 2090 & 2095 & 2100 & 2105 & 2110 & 2115\end{array}$ $\begin{array}{llllllllllllllllll}\text { ORF : } & V & R & T & \text { W } & \text { A } & \text { M } & \text { A } & \text { K } & \text { G } & \text { L } & \text { V } & \text { Q } & \text { H } & P & P & R\end{array}$

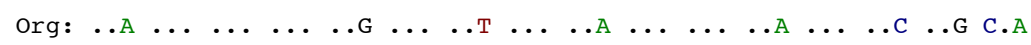
Opt: GTG AGG ACC TGG GCC ATG GCA AAA GGT CTG GTT CAG CAT CCT CCT AGG

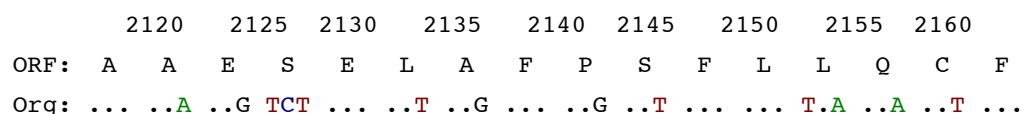
Opt: GCG GCT GAA AGC GAG CTC GCC TTC CCA AGC TTC CTT CTG CAG TGC TTC

$\begin{array}{llllllllll}2165 & 2170 & 2175 & 2180 & 2185 & 2190 & 2195 & 2200 & 2205 & 2210\end{array}$ $\begin{array}{lllllllllllllllllll}\text { ORF : } & \text { L } & G & A & \text { L } & \text { W } & \text { L } & \text { A } & \text { L } & \text { S } & \text { G } & \text { E } & \text { I } & \text { K } & \text { D } & \text { K } & \text { E }\end{array}$

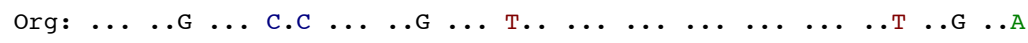
Opt: CTG GGT GCC TTG TGG CTT GCC CTG AGC GGC GAG ATC AAA GAC AAA GAG

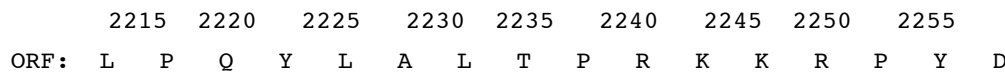

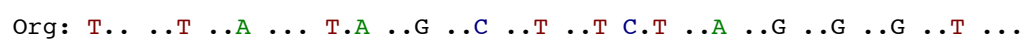
Opt: CTG CCA CAG TAC CTG GCA CTG ACC CCA AGg AAg AAA AGA CCT TAC GAC

\begin{tabular}{|c|c|c|c|c|c|c|c|c|c|c|c|c|c|c|c|}
\hline 2260 & 2265 & & 270 & 22 & 75 & 2280 & & 285 & & & 2295 & & 300 & 23 & 05 \\
\hline ORF : $\quad \mathrm{N}$ & W & $\mathrm{L}$ & $\mathrm{E}$ & G & $\mathrm{V}$ & $\mathrm{P}$ & $\mathrm{R}$ & $\mathrm{F}$ & L & A & G & L & I & F & $Q$ \\
\hline$g: \ldots T$ & $\cdots$ & .. & $\ldots G$ & $\ldots \mathrm{A}$ & $\ldots \mathrm{G}$ & $\ldots \mathrm{T}$ & C.. & $\cdots$ & Т.. & $\ldots \mathrm{T}$ & $\cdots$ & $\ldots \mathrm{T}$ & $\ldots \mathrm{A}$ & * & \\
\hline
\end{tabular}


$\begin{array}{llllllllll}2310 & 2315 & 2320 & 2325 & 2330 & 2335 & 2340 & 2345 & 2350 & 2355\end{array}$ ORF : $\begin{array}{llllllllllllllllllllllll}P & P & A & R & C & L & G & A & L & L & G & P & S & A & A & A\end{array}$

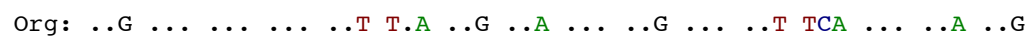
Opt: CCC CCT GCC AGg TGC CTG GGT GCC CTG CTC GGT CCA AGC GCA GCC GCT

$\begin{array}{lllllllll}2360 & 2365 & 2370 & 2375 & 2380 & 2385 & 2390 & 2395 & 2400\end{array}$

$\begin{array}{lllllllllllllllllll}\text { ORF : } & S & V & D & R & K & Q & K & V & L & A & R & Y & L & K & R & L\end{array}$

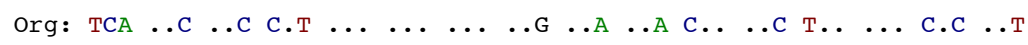
Opt: AGC GTG GAT AGg AAg CAg AAg GTC CTC GCC AGA TAT CTG AAG AGA CTG

\begin{tabular}{|c|c|c|c|c|c|c|c|c|c|c|c|c|c|c|c|c|}
\hline & 405 & & & 2415 & & 420 & & 25 & 2430 & & 435 & 24 & & 2445 & & 450 \\
\hline ORF : & $\mathrm{Q}$ & $\mathrm{P}$ & G & $\mathrm{T}$ & L & $\mathrm{R}$ & A & $\mathrm{R}$ & $Q$ & L & L & $\mathrm{E}$ & L & L & $\mathrm{H}$ & C \\
\hline Org: & $\ldots \mathrm{G}$ & $\cdots$ &. $\mathrm{T}$ & . C & T. A & C.A & $\cdots$ & C.C & . A & $\ldots A$ & $\ldots \mathrm{T}$ & $\ldots A$ & T.. & ..C & $\ldots \mathrm{T}$ & \\
\hline
\end{tabular}
Opt: CAA CCG GGA ACA CTG AGG GCT AGg CAG CTG CTG GAG CTG CTG CAC TGT

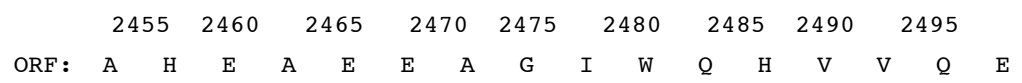
Org: $\ldots$. Opt: GCC CAC GAG GCC GAg GAG GCA GGC ATC TGG CAg CAC GTG GTG CAG GAG

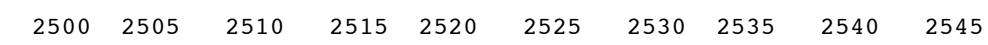
$\begin{array}{llllllllllllllllll}\text { ORF : } & L & P & G & R & L & S & F & L & G & T & R & L & T & P & P & D\end{array}$

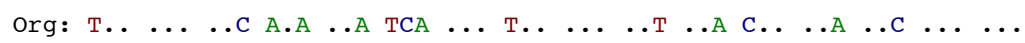
Opt: CTG CCT GGA CGC CTG AGC TTC CTG GGC ACA AGG TTG ACC CCG CCT GAT

$\begin{array}{llllllllll}2550 & 2555 & 2560 & 2565 & 2570 & 2575 & 2580 & 2585 & 2590 & 2595\end{array}$ ORF : $A \begin{array}{lllllllllllllllll} & \text { H } & \text { V } & \text { L } & \text { G } & \text { K } & \text { A } & \text { L } & \text { E } & \text { A } & \text { A } & \text { G } & \text { Q } & \text { D } & \text { F } & S\end{array}$ Org: $\ldots . . T \quad \ldots$. . Opt: GCC CAC GTG CTG GGC AAG GCG CTG GAG GCG GCC GGG CAG GAC TTT AGC

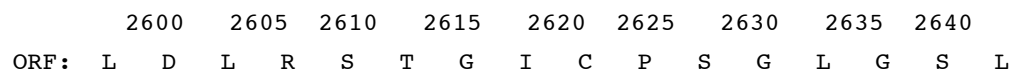

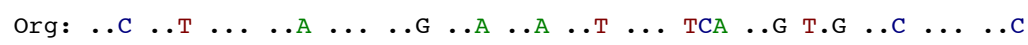
Opt: CTG GAC CTG AgG AgC ACT GGT ATC TGC CCA AGC GGA CTC GGg AgC CTG

$\begin{array}{llllllllll}2645 & 2650 & 2655 & 2660 & 2665 & 2670 & 2675 & 2680 & 2685 & 2690\end{array}$

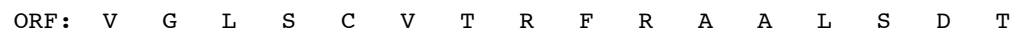

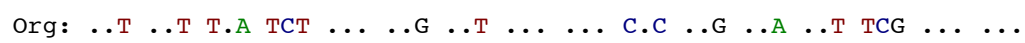
Opt: GTG GGG CTG AgC TGT GTC ACA AgG TTC AgG GCA GCC CTG AgC GAC ACT

\begin{tabular}{|c|c|c|c|c|c|c|c|c|c|c|c|c|c|c|c|c|}
\hline \multirow[b]{2}{*}{ ORF : } & \multicolumn{2}{|c|}{2695} & 2700 & \multicolumn{2}{|c|}{2705} & \multicolumn{2}{|c|}{2710} & 2715 & \multicolumn{2}{|c|}{2720} & \multicolumn{2}{|c|}{2725} & 2730 & \multicolumn{2}{|c|}{2735} & \\
\hline & V & A & L & W & E & $\mathrm{S}$ & L & $\mathrm{R}$ & $Q$ & $\mathrm{H}$ & G & $\mathrm{E}$ & $\mathrm{T}$ & $\mathrm{K}$ & L & L \\
\hline Org: & $\ldots C$ & $\ldots \mathrm{T}$ & . A & $\cdots$ & $\cdots$ & TCA & T.. & . & 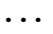 & $\ldots$ & $\ldots$ & $\cdots A$ & $\ldots T$ & $\ldots$ & $\ldots \mathrm{T}$ & 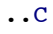 \\
\hline Opt: & GTT & $\mathrm{GCA}$ & CTG & TGG & GAA & AGC & CTG & CGT & CAG & CAC & GGA & GAG & $\mathrm{ACA}$ & AAA & СТC & STG \\
\hline
\end{tabular}


$\begin{array}{llllllllll}2740 & 2745 & 2750 & 2755 & 2760 & 2765 & 2770 & 2775 & 2780 & 2785\end{array}$ $\begin{array}{llllllllllllllllllll}\text { ORF : } & Q & A & A & E & E & K & F & T & I & E & P & F & K & A & K & S\end{array}$

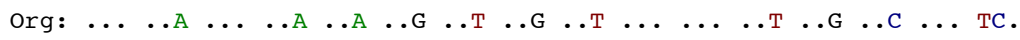
Opt: CAA GCG GCT GAG GAG AAA TTC ACC ATC GAG CCT TTC AAA GCG AAA AGC

$\begin{array}{llllllllll}2790 & 2795 & 2800 & 2805 & 2810 & 2815 & 2820 & 2825 & 2830 & 2835\end{array}$ $\begin{array}{lllllllllllllllllll}\text { ORF : } & L & K & D & V & E & D & L & G & K & L & V & Q & T & Q & R & T\end{array}$

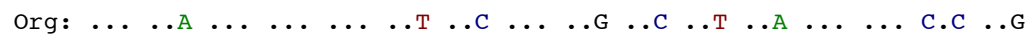
Opt: CTG AAG GAC GTG GAG GAC CTG GGC AAA CTG GTG CAG ACG CAG AGG ACT

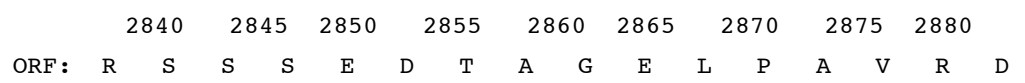

Org: А.А $\ldots$ Т $\ldots$. С $\ldots$. Opt: CGC TCA TCT AGC GAA GAT ACA GCC GGC GAG TTG CCA GCA GTG AgG GAC

$\begin{array}{llllllllll}2885 & 2890 & 2895 & 2900 & 2905 & 2910 & 2915 & 2920 & 2925 & 2930\end{array}$ $\begin{array}{lllllllllllllllllll}\text { ORF : } & L & K & K & \text { L } & E & F & A & L & G & P & V & S & G & P & Q & A\end{array}$

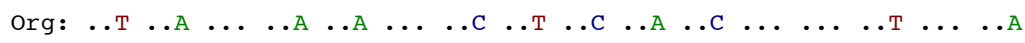
Opt: CTG AAg AAA CTG GAG TTC GCA CTG GGT CCT GTT AGC GGA CCA CAA GCC

\begin{tabular}{lllllllllllllllll} 
& \multicolumn{8}{c}{2935} & 2940 & 2945 & 2950 & 2955 & 2960 & 2965 & 2970 & 2975 \\
ORF : & F & P & K & L & V & R & I & L & T & A & F & S & S & L & Q & H \\
Org: & T & & & & & & &
\end{tabular} Opt: TTC CCG AAG CTT GTG AGg ATC CTG ACC GCG TTT AGC AGC CTG CAA CAC

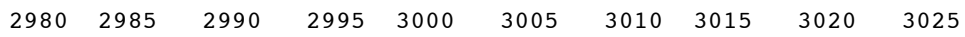

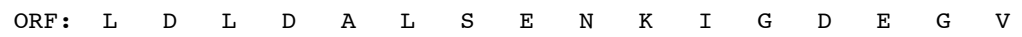

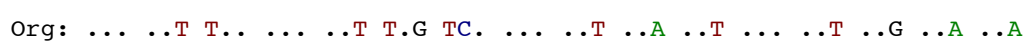
Opt: CTG GAC CTG GAC GCC CTT AGC GAG AAC AAG ATA GGA GAC GAA GGC GTG

$\begin{array}{llllllllll}3030 & 3035 & 3040 & 3045 & 3050 & 3055 & 3060 & 3065 & 3070 & 3075\end{array}$ $\begin{array}{lllllllllllllllll}\text { ORF : } & S & Q & L & S & A & T & F & P & Q & L & K & S & L & E & T & L\end{array}$ Org: ТС. ..А $\ldots$ Т ТСА $\ldots . . . G \quad \ldots$ T $\ldots$. Opt: AGC CAG CTG AGC GCT ACC TTC CCA CAG CTG AAA AGC CTG GAA ACG CTG $\begin{array}{lllllllll}3080 & 3085 & 3090 & 3095 & 3100 & 3105 & 3110 & 3115 & 3120\end{array}$

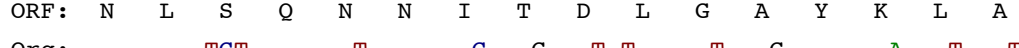
Opt: AAT CTT AGC CAG AAC AAC ATA ACC GAC CTG GGC GCC TAC AAG CTG GCG

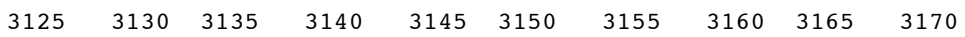
ORF: $\begin{array}{llllllllllllllllll} & \mathrm{A} & \mathrm{A} & \mathrm{L} & \mathrm{P} & \mathrm{S} & \mathrm{L} & \mathrm{A} & \mathrm{A} & \mathrm{S} & \mathrm{L} & \mathrm{L} & \mathrm{R} & \mathrm{L} & \mathrm{S} & \mathrm{L} & \mathrm{Y}\end{array}$

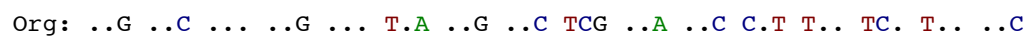
Opt: GAA GCA CTT CCT AGC CTG GCC GCT AGC CTG CTG AGA CTG AGT CTG TAT 


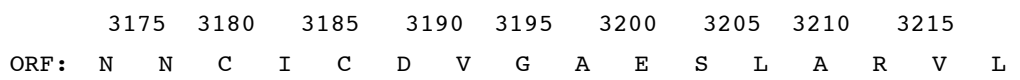

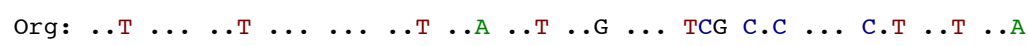
opt: AAC AAC TGC ATC TGT GAC GTT GGC GCC GAA AGC TTG GCC AGG GTG CTC

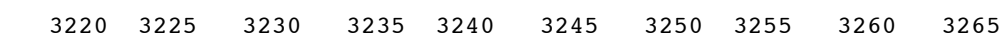
$\begin{array}{llllllllllllllllllll}\text { ORF : } & P & D & M & V & S & L & R & V & M & D & V & Q & Y & N & K & F\end{array}$

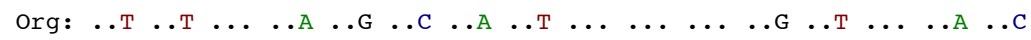
Opt: CCC GAC ATG GTG TCA CTG CGT GTG ATG GAC GTG CAA TAC AAC AAG TTT

$\begin{array}{llllllllll}3270 & 3275 & 3280 & 3285 & 3290 & 3295 & 3300 & 3305 & 3310 & 3315\end{array}$

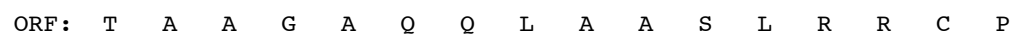

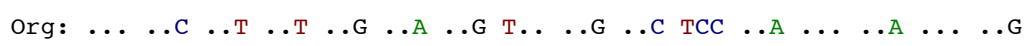
Opt: ACA GCG GCC GGG GCA CAG CAA CTG GCT GCG AGT CTG AGG AGG TGT CCT

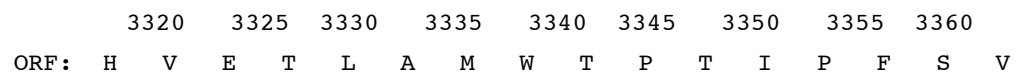

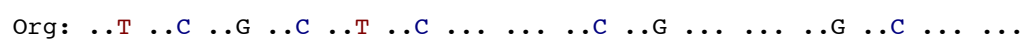
Opt: CAC GTG GAA ACT CTG GCA ATG TGG ACT CCT ACC ATC CCC tTt AGC GTC

$\begin{array}{llllllllll}3365 & 3370 & 3375 & 3380 & 3385 & 3390 & 3395 & 3400 & 3405 & 3410\end{array}$ $\begin{array}{lllllllllllllllll}\text { ORF : } & Q & E & H & L & Q & Q & Q & D & S & R & I & S & L & R & V & K\end{array}$

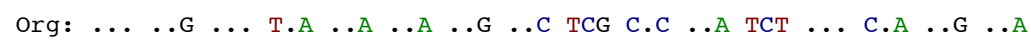
Opt: CAG GAA CAC CTT CAG CAG CAA GAT AGC AGG ATC AGC CTG AGG GTC AAG

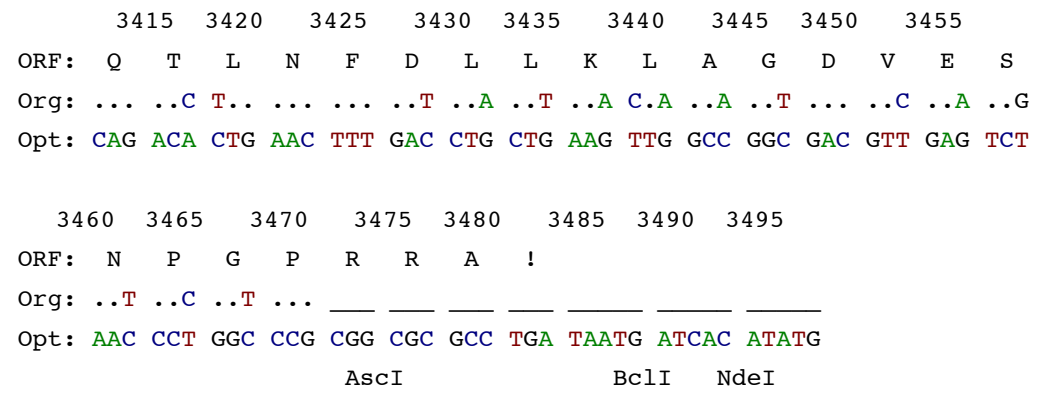


1.1.1 Restriction table

\begin{tabular}{|l|l|l|l|}
\hline Enzyme & Recognition & Frequency & Positions \\
\hline Ascl & gg'cgcg_cc & 1 & 3472 \\
\hline Bcll & t'gatc_a & 1 & 3486 \\
\hline EcoRl & g'aatt_c & 1 & 6 \\
\hline Ndel & ca'ta_tg & 1 & 3492 \\
\hline Xmal & c'ccgg_g & 1 & 0 \\
\hline
\end{tabular}

1.1.2 Enzymes that cut five or fewer times

\begin{tabular}{|l|l|l|l|}
\hline Enzyme & Recognition & Frequency & Positions \\
\hline Ascl & gg'cgcg_cc & 1 & 3472 \\
\hline Bcll & t'gatc_a & 1 & 3486 \\
\hline EcoRl & g'aatt_c & 1 & 6 \\
\hline Ndel & ca'ta_tg & 1 & 3492 \\
\hline Xmal & c'ccgg_g & 1 & 0 \\
\hline
\end{tabular}

\subsubsection{Enzymes that do not cut}

None.

\subsection{Dotplot}

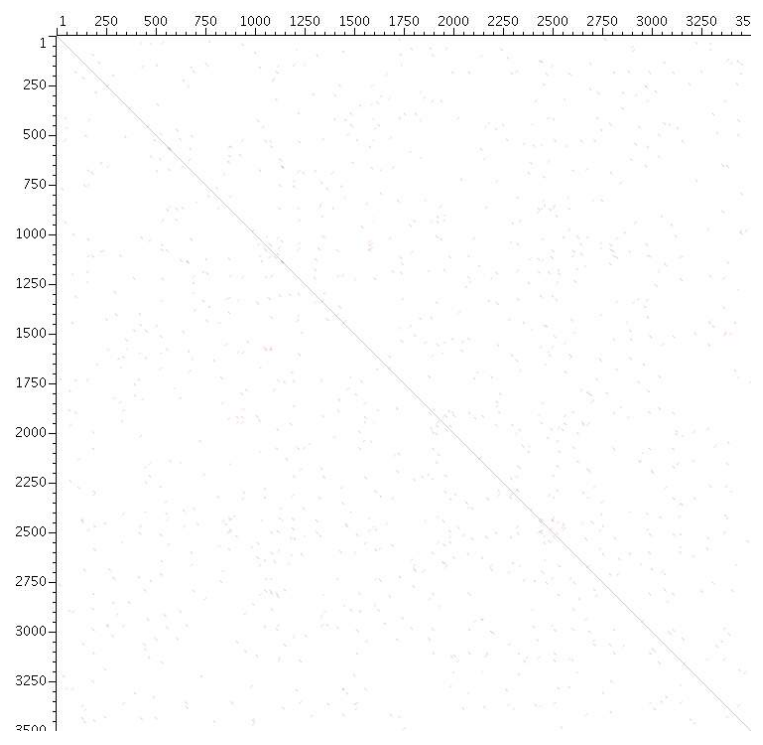




\subsection{ORFs}

The gene was optimized using the following ORF(s). Please note that sequence positions are given relative to the continuous DNA sequence defined by all ORFs:

ORF: $16-3483$

\section{Optimized parameters}

\subsection{Codon Usage}

This is a comparison of the codon frequency of the original sequence vs. the optimized sequence. As a reference, the codon usage of the target organism is included. The charts indicate the sum of the deviation from the target codon usage, for the original and the optimized sequence. Lower bars indicate a better match with the target codon usage.

2.1.1 A - Alanine:

$\begin{array}{ll}\text { Codon } & \text { Original } \\ \text { GCU } & 27.9 \% \\ \text { GCC } & 22.5 \% \\ \text { GCA } & 19.8 \% \\ \text { GCG } & 29.7 \%\end{array}$

Target

$26.0 \%$

$40.0 \%$

$22.0 \%$

Optimized

$21.6 \%$

$40.5 \%$

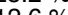

$12.0 \%$

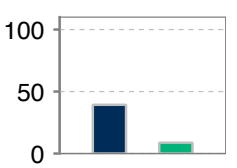

2.1.2 C - Cysteine:

Codon

UGU

Original

$36.0 \%$

Target

$55.0 \%$

Optimized

$44.0 \%$

$56.0 \%$

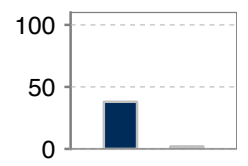

2.1.3 D - Aspartic acid:

Codon
GAU
GAC

Original

$49.1 \%$

Target
$46.0 \%$

$54.0 \%$

Optimized
$28.1 \%$
$71.9 \%$

GAC

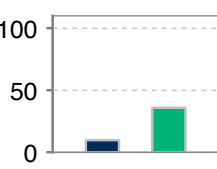

2.1.4 E - Glutamic acid:

$\begin{array}{rrr}\text { Codon } & \text { Original } & \text { Target } \\ \text { GAA } & 50.0 \% & 42.0 \% \\ \text { GAG } & 50.0 \% & 58.0 \%\end{array}$

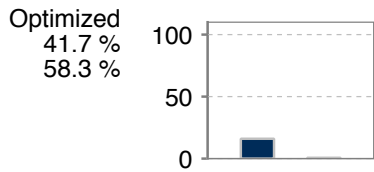


2.1.5 F - Phenylalanine:

$\begin{array}{rrrr}\text { Codon } & \text { Original } & \text { Target } & \text { Optimized } \\ \text { UUU } & 52.5 \% & 46.0 \% & 30.0 \% \\ \text { UUC } & 47.5 \% & 54.0 \% & 70.0 \%\end{array}$

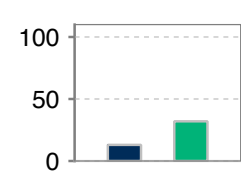

2.1.6 G - Glycine:

$\begin{array}{ccc}\text { Codon } & \text { Original } & \text { Targ } \\ \text { GGU } & 22.5 \% & 16.0 \\ \text { GGC } & 23.8 \% & 33.0 \\ \text { GGA } & 31.2 \% & 25.0 \\ \text { GGG } & 22.5 \% & 26.0 \%\end{array}$

$\begin{array}{ll}16.2 \% \\ 37.0 \% & 37.5 \%\end{array}$

$26.0 \%$

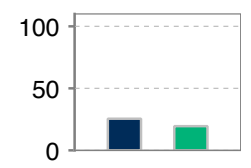

2.1.7 H - Histidine:

Codon

CAC

Original
$41.7 \%$
$58.3 \%$

Target
$41.0 \%$

$41.0 \%$

Optimized
$4.2 \%$
$95.8 \%$

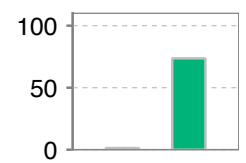

2.1.8 I - Isoleucine:

$\begin{array}{rrr}\text { Codon } & \text { Original } & \text { Targe } \\ \text { AUU } & 25.9 \% & 35.0 \% \\ \text { AUC } & 37.0 \% & 47.0 \% \\ \text { AUA } & 37.0 \% & 18.0 \%\end{array}$

$35.0 \%$
$47.0 \%$

$18.0 \%$

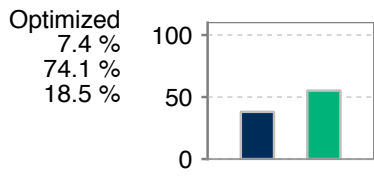

2.1.9 K - Lysine:

$\begin{array}{rrr}\text { Codon } & \text { Original } & \text { Target } \\ \text { AAA } & 61.4 \% & 43.0 \% \\ \text { AAG } & 38.6 \% & 57.0 \%\end{array}$

Optimized $43.2 \%$
$56.8 \%$

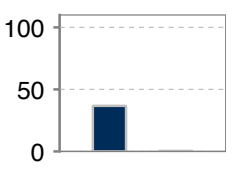

2.1.10 L - Leucine:

Codon
UUA
UUG
CUU
CUC
CUA
CUG

Original
$13.4 \%$
$19.2 \%$
$17.4 \%$
$16.3 \%$
$15.1 \%$
$18.6 \%$

Target $0.0 \%$

$12.0 \%$

$13.0 \%$

$19.0 \%$

$0.0 \%$

$56.0 \%$

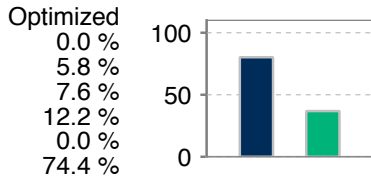


2.1.11 M - Methionine:

$\begin{array}{crrr}\text { Codon } & \text { Original } & \text { Target } & \text { Optimized } \\ \text { AUG } & 100.0 \% & 100.0 \% & 100.0 \%\end{array}$

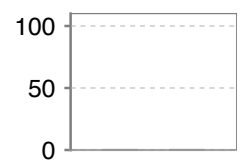

2.1.12 N - Asparagine:

Codon

AAU
Original

$44.4 \%$

$44.4 \%$
Optimized $22.2 \%$ $77.8 \%$

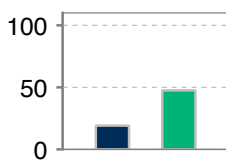

2.1.13 P - Proline:

\begin{tabular}{rr} 
Codon & Original \\
CCU & $30.0 \%$ \\
CCC & $22.2 \%$ \\
CCA & $17.8 \%$ \\
\hline
\end{tabular}

Target
$28.0 \%$
$32.0 \%$
$27.0 \%$
$13.0 \%$
Optimized $28.9 \%$ $32.2 \%$ $26.7 \%$
$12.2 \%$

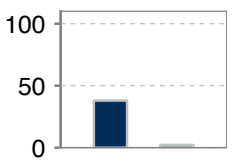

2.1.14 Q - Glutamine:

$$
\text { Codon }
$$

CAA

Original
$50.8 \%$

$50.8 \%$
$49.2 \%$

Target
$26.0 \%$

$26.0 \%$

Optimized $25.4 \%$
$74.6 \%$

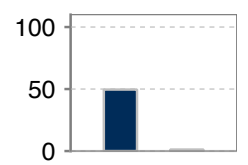

2.1.15 R - Arginine:

$\begin{array}{cc}\text { Codon } & \text { Original } \\ \text { CGU } & 17.2 \% \\ \text { CGC } & 25.0 \% \\ \text { CGA } & 15.6 \% \\ \text { CGG } & 12.5 \% \\ \text { AGA } & 14.1 \% \\ \text { AGG } & 15.6 \%\end{array}$

Target
$8.0 \%$
$18.0 \%$
$10.0 \%$
$20.0 \%$
$21.0 \%$
$23.0 \%$

Optimized

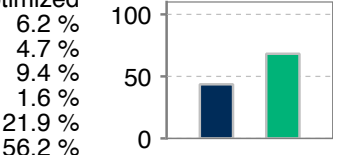

$56.2 \%$

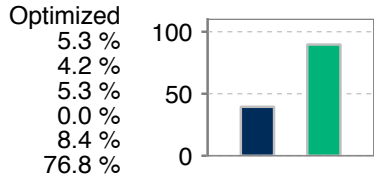

2.1.16 S - Serine:

Codon
UCU
UCC
UCA
UCG
AGU
AGC

$$
\begin{aligned}
& \text { Original } \\
& 14.7 \% \\
& 15.8 \% \\
& 18.9 \% \\
& 15.8 \% \\
& 11.6 \% \\
& 23.2 \%
\end{aligned}
$$

Target $18.0 \%$ $21.0 \%$ $15.0 \%$ $14.0 \%$ $32.0 \%$ 
2.1.17 T - Threonine:

$\begin{array}{rr}\text { Codon } & \text { Origina } \\ \text { ACU } & 32.1 \% \\ \text { ACC } & 20.8 \% \\ \text { ACA } & 20.8 \%\end{array}$

Target

$26.4 \% \quad 13.0 \%$

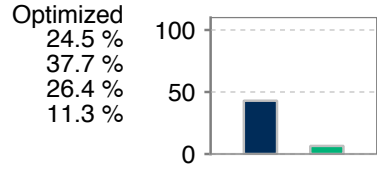

2.1.18 V - Valine:

Codon

GUU

GUC

GUA

$28.6 \%$

$23.2 \%$

(3.

Target

$18.0 \%$

$23.0 \%$

$5.0 \%$

Optimized

$17.9 \%$
$12.5 \%$

$12.5 \%$

$69.6 \%$

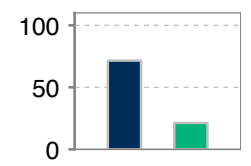

2.1.19 W - Tryptophan:

Codon

Original

$100.0 \%$

Target
$100.0 \%$

Optimized
$100.0 \%$

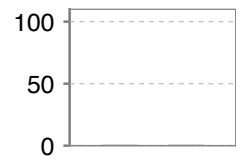

2.1.20 Y - Tyrosine:

$\begin{array}{lrr}\text { Codon } & \text { Original } & \text { Target } \\ \text { UAU } & 54.5 \% & 44.0 \% \\ \text { UAC } & 45.5 \% & 56.0 \%\end{array}$

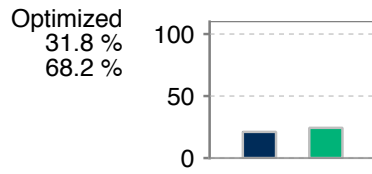

2.1.21 ! - Stop:

$\begin{array}{rrr}\text { Codon } & \text { Original } & \text { Target } \\ \text { UAA } & 0.0 \% & 30.0 \% \\ \text { UGA } & 100.0 \% & 45.0 \% \\ \text { UAG } & 0.0 \% & 25.0 \%\end{array}$

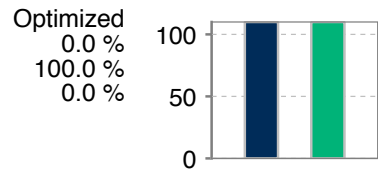

\subsection{Secondary structure}

In general, an extended secondary structure of the mRNA can interfere with the translation process.

Therefore, the gene optimization tries to reduce the number and length of potential hairpin loops. 
2.2.1 Original sequence

\begin{tabular}{|l|l|}
\hline Number of helices & 88 \\
\hline Average helix length & 6.69 \\
\hline Maximum helix length & 20 \\
\hline Average helix score & 7.80 \\
\hline
\end{tabular}

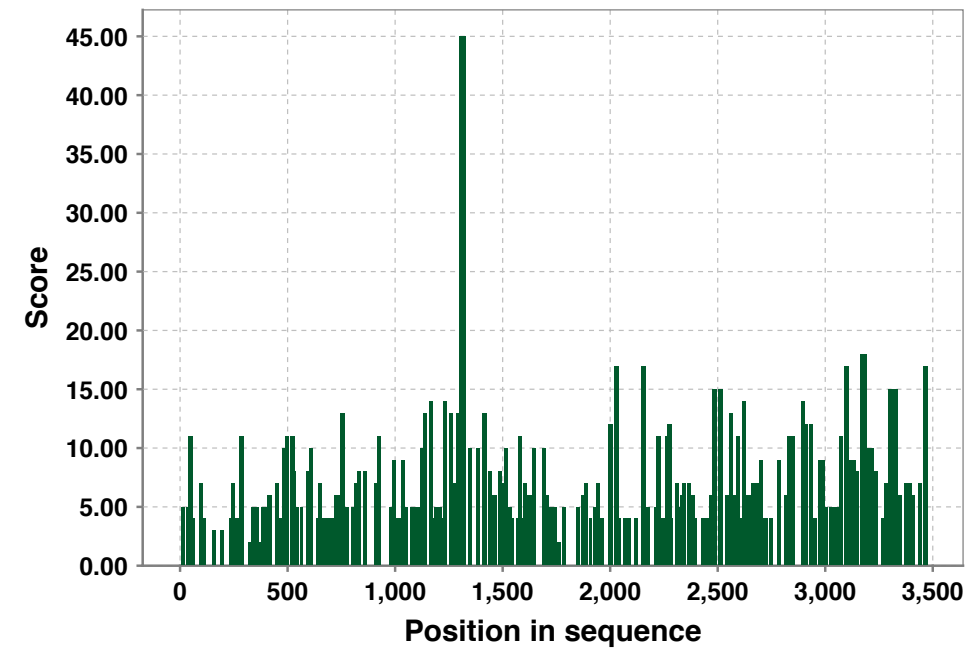

2.2.2 Optimized sequence

\begin{tabular}{|l|l|}
\hline Number of helices & 70 \\
\hline Average helix length & 5.74 \\
\hline Maximum helix length & 11 \\
\hline Average helix score & 5.70 \\
\hline
\end{tabular}

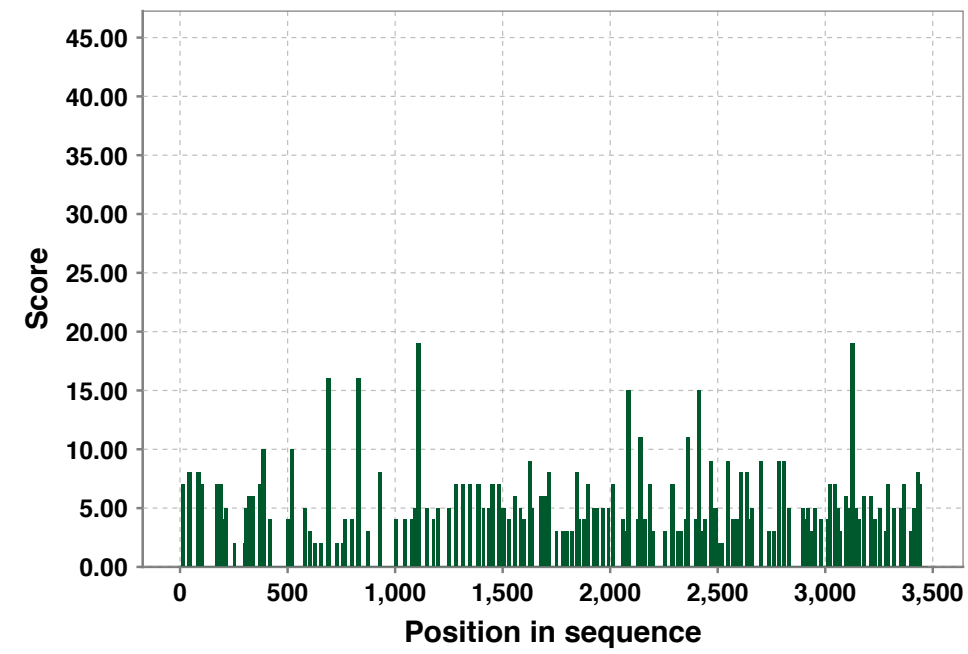




\subsection{GC distribution}

GC ratio over a window of $31 \mathrm{nt}$ :

Original sequence: $53.32 \%(29.03 \%-77.42 \%)$

Optimized sequence: $59.66 \%$ (35.48\% - 80.65\%)

The number indicate the average GC content. The values in brackets indicate the interval containing $95 \%$ of all nucleotides if the GC content is averaged over a window of 11 nucleotides.

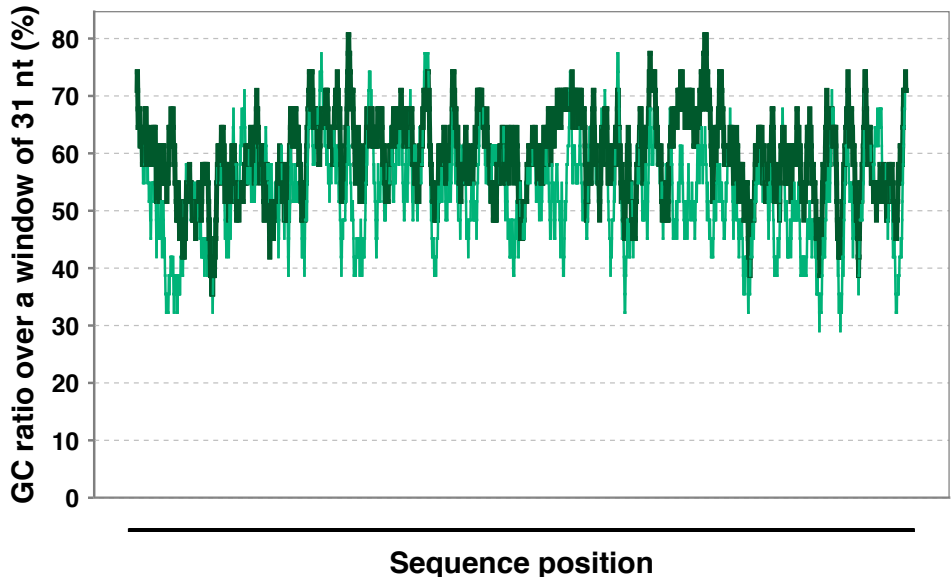

Original sequence - Optimized sequence

\subsection{Secondary ORFs}

During the optimization, secondary ORFs were removed. These are ORFs in the second or third frame or on the opposite strand. The removal was done by inserting stop codons by silent mutagenesis, or by changing start codons. The optimization tries to avoid secondary ORFs with more than 50 nucleotides.

\subsubsection{Original sequence:}

Number of secondary ORFs: 67

Total length of sec. ORFs: 1768

Number of ORFs with >50nt: 10

\subsubsection{Optimized sequence:}

Number of secondary ORFs: 26

Total length of sec. ORFs: 831

Number of ORFs with >50nt: 3 


\subsection{Restriction sites}

The table lists the unwanted restriction sites contained in the sequence, as well as their positions.

\begin{tabular}{|l|l|l|}
\hline \multicolumn{1}{|c|}{ Enzyme } & \multicolumn{1}{|c|}{$\begin{array}{c}\text { Positions in original } \\
\text { sequence }\end{array}$} & \multicolumn{1}{c|}{$\begin{array}{c}\text { Positions in optimized } \\
\text { sequence }\end{array}$} \\
\hline Ascl & 1 & 1 \\
\hline Bcll & 1 & 0 \\
\hline EcoRl & 1 & 0 \\
\hline Ndel & 0 & 0 \\
\hline Xmal & 0 & 0 \\
\hline
\end{tabular}

\subsection{Cryptic splice sites}

The following table shows possible splice sites in the specified sequence. The score is relative to the range between the best-scoring and worst-scoring splice sites in the training set.

\subsubsection{Splice sites in original sequence}

\begin{tabular}{|c|c|c|}
\hline Type & Position & Relevance \\
\hline Acceptor & 30 & Likely \\
\hline Acceptor & 479 & Possible \\
\hline Acceptor & 1004 & Likely \\
\hline Acceptor & 1415 & Possible \\
\hline Acceptor & 1749 & Possible \\
\hline Acceptor & 2333 & Likely \\
\hline Acceptor & 2812 & Possible \\
\hline Acceptor & 3120 & Possible \\
\hline Acceptor & 3176 & Possible \\
\hline Donor & 274 & Possible \\
\hline Donor & 795 & Possible \\
\hline Donor & 978 & Possible \\
\hline Donor & 1197 & Possible \\
\hline Donor & 2052 & Likely \\
\hline Donor & 2224 & Possible \\
\hline Donor & 2303 & Likely \\
\hline Donor & 2339 & Likely \\
\hline Donor & 2898 & Possible \\
\hline
\end{tabular}

\subsubsection{Splice sites in optimized sequence}

\begin{tabular}{|l|l|l|}
\hline \multicolumn{1}{|c|}{ Type } & \multicolumn{1}{|c|}{ Position } & \multicolumn{1}{c|}{ Relevance } \\
\hline Donor & 312 & Possible \\
\hline Donor & 978 & Likely \\
\hline Donor & 1739 & Likely \\
\hline Donor & 2052 & Possible \\
\hline Donor & 2303 & Likely \\
\hline Donor & 2898 & Possible \\
\hline Donor & 2928 & Possible \\
\hline
\end{tabular}




\subsection{AT/GC stretch restrictor}

\subsubsection{Original sequence}

Number of stretches longer than 4 nt: 107

Average AT/GC stretch length: 5.83

\subsubsection{Optimized sequence}

Number of stretches longer than $4 \mathrm{nt}: 55$

Average AT/GC stretch length: 6.13

\section{Optimized sequence}

This is the optimized sequence, ready for copying and pasting into other applications.

CCCGG GGAAT TCACC ATGGG GTGTC TCGCA CCCCG ACCGG CAGGC AGTTA TCTCA GCGAA CCCCA GGGAA GCAGC CAGTG CGCAA CTATG GAGCT GGGAC CCTTG GAAGG CGGCT ATCTC GAGCT GCTGA ATAGC GACGC TGATC CCCTG TGCCT GTACC ACTTC TACGA CCAGA TGGAC CTGGC CGGGG AGGAA GAGAT CGAGC TGTAT AGCGA ACCGG ATACC GATAC CATCA ACTGT GACCA GTTTA GCAGA CTGCT GTGCG ACATG GAAGG TGACG AAGAG ACAAG AGAAG CCTAC GCTAA CATCG CAGAG TTGGA CCAGT ACGTG TTCCA GGACT CCCAA CTGGA GGGTC TTAGC AAAGA TATCT TCAAA CACAT CGGTC CTGAT GAGGT GATCG GGGAG AGCAT GGAGA TGCCT GCCGA AGTGG GACAG AAAAG CCAGA AGAGA CCCTT CCCGG AAGAA CTGCC AGCGG ATCTG AAACA CTGGA AACCA GCTGA ACCCC CAACT GTTGT GACTG GAAGC CTGCT GGTCG GACCT GTTAG CGACT GCAGC ACCTT GCCTT GCCTT CCCCT CCCCG CACTG TTCAA TCAAG AGCCA GCCAG CGGAC AAATG AGACT GGAGA AGACA GACCA GATAC CTATG CCCTT TAGCA GCAGC AGTCT GTCTT GCCTG AACCT CCCAG AAGGA CCTAT TCAGT TCGTG CCCAC CATTT CAACG CTCCC CCACG GCCTG TGGCA GATAA GCGAG GCTGG GACAG GCGTG TCTAG CATAT TCATC TACCA CGGCG AGGTT CCCCA GGCGA GTCAG GTGCC GCCAC CAAGC GGCTT CACAG TGCAC GGACT GCCCA CAAGT CCAGA CAGGC CAGGC AGCAC CTCCC CTTTC GCGCC TAGCG CTACT GACCT TCCCA GCATG CCAGA GCCCG CCCTG ACCAG CAGGG CTAAC ATGAC CGAAC ACAAG ACGAG CCCCA CTCAG TGCCC CGCCG CCGGC GAAGT GAGCA ACAAG CTGCC CAAGT GGCCT GAACC AGTGG AGCAG TTCTA CAGGA GCCTG CAGGA CACTT ACGGA GCCGA GCCTG CAGGA CCTGA CGGTA TCCTG GTGGA GGTGG ACCTG GTGCA GGCTA GGCTG GAGAg GAGCA GCAGC AAAAg CCTGG AAAGA GAGCT TGCGA CACCC GACTG GGCAG AAAGG CAGCT GGCAC AGGGT GGACT GGCTG AGGTC CTGCT GGCCG CTAAG GAGCA CAGAA GGCCC AGGGA GACGA GGGTG ATCGC TGTGC TCGGA AAAGC AGGAC AAGGG AAGTC CTACT GGGCT GGAGC TGTGA GCCGT GCCTG GGCTT GTGGC AGGCT GCCCC AGTAC GATTT CGTGT TTAGC GTTCC GTGCC ACTGT CTGAA TAGGC CAGGA GACGC 
CTACG GCTTG CAAGA CCTCC TGTTT AGCCT GGGAC CGCAG CCACT GGTGG CTGCC GACGA GGTGT TCAGC CACAT CCTGA AGAGA CCCGA TAGGG TGCTC CTGAT CCTGG ACGCC TTTGA GGAAC TGGAG GCACA AGACG GCTTC CTGCA CAGCA CCTGT GGGCC CGCTC CTGCA GAACC CTGCA GTCTG AGAGG CCTGC TGGCA GGACT GTTCC AGAAG AAACT CCTGC GAGGT TGTAC CCTTC TTCTG ACCGC TCGAC CTCGA GGAAG ACTGG TCCAG TCACT GAGCA AGGCA GACGC CCTGT TTGAG CTGAG CGGCT TCAGC ATGGA GCAGG CACAA GCCTA TGTGA TGAGG TATTT CGAGA GCAGC GGGAT GACTG AACAC CAGGA TCGAG CCCTG ACCCT GTTGA GGGAT CGACC ACTCC TCCTG TCTCA CTCCC ACAGT CCAAC ACTGT GCAGG GCCGT GTGTC AGCTG AGCGA AGCCC TCCTG GAACT GGGCG AAGAC GCCAA GCTGC CCAGC ACCCT CACTG GCCTG TACGT GGGGC TGCTG GGCAG AGCTG CCCTG GATAG CCCAC CTGGT GCCCT GGCGG AACTG GCCAA GCTGG CATGG GAGCT CGGCA GGCGT CACCA GTCAA CGCTG CAGGA AGATC AATTC CCGAG CGCAG ACGTG AGGAC CTGGG CCATG GCAAA AGGTC TGGTT CAGCA TCCTC CTAGG GCGGC TGAAA GCGAG CTCGC CTTCC CAAGC TTCCT TCTGC AGTGC TTCCT GGGTG CCTTG TGGCT TGCCC TGAGC GGCGA GATCA AAGAC AAAGA GCTGC CACAG TACCT GGCAC TGACC CCAAG GAAGA AAAGA CCTTA CGACA ACTGG CTGGA AGGCG TTCCC AGGTT CCTGG CAGGG CTGAT CTTTC AACCC CCTGC CAGGT GCCTG GGTGC CCTGC TCGGT CCAAG CGCAG CCGCT AGCGT GGATA GGAAG CAGAA GGTCC TCGCC AGATA TCTGA AGAGA CTGCA ACCGG GAACA CTGAG GGCTA GGCAG CTGCT GGAGC TGCTG CACTG TGCCC ACGAG GCCGA GGAGG CAGGC ATCTG GCAGC ACGTG GTGCA GGAGC TGCCT GGACG CCTGA GCTTC CTGGG CACAA GGTTG ACCCC GCCTG ATGCC CACGT GCTGG GCAAG GCGCT GGAGG CGGCC GGGCA GGACT TTAGC CTGGA CCTGA GGAGC ACTGG TATCT GCCCA AGCGG ACTCG GGAGC CTGGT GGGGC TGAGC TGTGT CACAA GGTTC AGGGC AGCCC TGAGC GACAC TGTTG CACTG TGGGA AAGCC TGCGT CAGCA CGGAG AGACA AAACT CCTGC AAGCG GCTGA GGAGA AATTC ACCAT CGAGC CTTTC AAAGC GAAAA GCCTG AAGGA CGTGG AGGAC CTGGG CAAAC TGGTG CAGAC GCAGA GGACT CGCTC ATCTA GCGAA GATAC AGCCG GCGAG TTGCC AGCAG TGAGG GACCT GAAGA AACTG GAGTT CGCAC TGGGT CCTGT TAGCG GACCA CAAGC CTTCC CGAAG CTTGT GAGGA TCCTG ACCGC GTTTA GCAGC CTGCA ACACC TGGAC CTGGA CGCCC TTAGC GAGAA CAAGA TAGGA GACGA AGGCG TGAGC CAGCT GAGCG CTACC TTCCC ACAGC TGAAA AGCCT GGAAA CGCTG AATCT TAGCC AGAAC AACAT AACCG ACCTG GGCGC CTACA AGCTG GCGGA AGCAC TTCCT AGCCT GGCCG CTAGC CTGCT GAGAC TGAGT CTGTA TAACA ACTGC ATCTG TGACG TTGGC GCCGA AAGCT TGGCC AGGGT GCTCC CCGAC ATGGT GTCAC TGCGT GTGAT GGACG TGCAA TACAA CAAGT TTACA GCGGC CGGGG CACAG CAACT GGCTG CGAGT CTGAG GAGGT GTCCT CACGT GGAAA CTCTG GCAAT GTGGA CTCCT ACCAT CCCCT TTAGC GTCCA GGAAC ACCTT CAGCA GCAAG ATAGC AGGAT CAGCC TGAGG GTCAA GCAGA CACTG AACTT TGACC TGCTG AAGTT GGCCG GCGAC GTTGA GTCTA ACCCT GGCCC GCGGC GCGCC TGATA ATGAT CACAT ATG

Created with Leto 1.0 - the dedicated software for gene synthesis and optimized protein expression. To get more information on Leto and download a demo version, visit www.entelechon.com/leto or call Entelechon at +499419468360. 
B - Publications and submitted articles

Non-immune cells differentially regulate MHC class II expression by modulating the proteasomal degradation of class II transactivator. Palma ML, Douradinha B, Duangkhae P, Viana IFT, Rigato PO, Dhalia R, Mailliard RB, Nascimento EJM, Oshiro TM, Duarte AJS, Marques ETA. J Biol Chem. (Submitted)

HIV vaccine development: Tools and knowledge. Rigato PO, Palma ML. OMICs Ebook Group. (Book chapter)

Probiotic Saccharomyces cerevisae strains as biotherapeutic tools: is there room for improvement? Palma ML, Zamith-Miranda D, Martins FS, Bozza FA, Nimrichter L, Montero-Lomeli M, Marques ETA, Douradinha B. Appl Microbiol Biot.

Lipid droplet levels vary heterogeneously in response to simulated gastrointestinal stresses in different probiotic Saccharomyces strains. Zamith-Miranda D*, Palma ML*, Matos GS, Schiebel JG, Maya-Montero CM, Aronovich M, Bozza PT, Bozza FA, Nimrichter L, Masuda CA, Montero-Lomeli M, Marques ETA, Martins FS, Douradinha B. J Funct Foods (In review) 


\section{REFERENCES}




\section{REFERENCES*}

1. Touraine JL, Bétuel H. The bare lymphocyte syndrome: immunodeficiency resulting from the lack of expression of HLA antigens. Birth Defects Orig Artic Ser 1983;19:83-5.

2. Steimle V, Otten LA, Zufferey M, Mach B. Complementation cloning of an MHC class II transactivator mutated in hereditary MHC class II deficiency (or bare lymphocyte syndrome). Cell 1993;75:135-46.

3. Reith W, LeibundGut-Landmann S, Waldburger JM. Regulation of MHC class II gene expression by the class II transactivator. Nat Rev Immunol 2005;5:793806.

4. Krawczyk M, Reith W. Regulation of MHC class II expression, a unique regulatory system identified by the study of a primary immunodeficiency disease. Tissue Antigens 2006;67:183-97.

5. Kern I, Steimle V, Siegrist C-A, Mach B. The two novel MHC class II transactivators RFX5 and CIITA both control expression of HLA-DM genes. Int Immunol 1995;7:1295-9.

6. Westerheide SD, Louis-Plence P, Ping D, He XF, Boss JM. HLA-DMA and HLA-DMB gene expression functions through the conserved $\mathrm{S}-\mathrm{X}-\mathrm{Y}$ region. $J$ Immunol 1997;158:4812-21.

7. Taxman DJ, Cressman DE, Ting JP. Identification of class II transcriptional activator-induced genes by representational difference analysis: discoordinate regulation of the DN alpha/DO beta heterodimer. J Immunol 2000;165:1410-6.

8. Benoist C, Mathis D. Regulation of major histocompatibility complex class-II genes: X, Y and other letters of the alphabet. Annu Rev Immunol 1990;8:681715 .

9. Krawczyk M, Seguín-Estévez Q, Leimgruber E, Sperisen P, Schmid C, Bucher $\mathrm{P}$, et al. Identification of CIITA Regulated Genetic Module Dedicated for Antigen Presentation. PLoS Genet 2008;4:e1000058.

10. Choi NM, Majumder P, Boss JM. Regulation of major histocompatibility complex class II genes. Curr Opin Immunol 2011;23:81-7.

11. Masternak K, Muhlethaler-Mottet A, Villard J, Zufferey M, Steimle V, Reith W. CIITA is a transcriptional coactivator that is recruited to MHC class II promoters by multiple synergistic interactions with an enhanceosome complex.

*According to:

Adapted from the International Committee of Medical Journals Editors (Vancouver)

University of São Paulo. Medical School. Library and Documentation Service. Guia de apresentação de dissertações, teses e monografias da FMUSP. Prepared by Anneliese Carneiro da Cunha, Maria Julia A.L. Freddi, Maria F. Crestana, Marinalva de S. Aragão, Suely C. Cardoso, Valéria Vilhena. 3a ed.

São Paulo: Library and Documentation Division; 2011.

Journals title abbreviation according to List of Journals Indexed in Index Medicus. 
Genes Dev 2000;14:1156-66.

12. Zhu XS, Linhoff MW, Li G, Chin KC, Maity SN, Ting JP. Transcriptional scaffold: CIITA interacts with NF-Y, RFX, and CREB to cause stereospecific regulation of the class II major histocompatibility complex promoter. Mol Cell Biol 2000;20:6051-61.

13. Kobayashi KS, van den Elsen PJ. NLRC5: a key regulator of MHC class Idependent immune responses. Nat Rev Immunol 2012;12:813-20.

14. Fontes JD, Jiang B, Peterlin BM. The class II trans-activator CIITA interacts with the TBP-associated factor TAFII32. Nucl Acids Res 1997;25:2522-8.

15. Mahanta SK, Scholl T, Yang FC, Strominger JL. Transactivation by CIITA, the type II bare lymphocyte syndrome-associated factor, requires participation of multiple regions of the TATA box binding protein. Proc Natl Acad Sci USA 1997;94:6324-9.

16. Kanazawa S, Okamoto T, Peterlin BM. Tat Competes with CIITA for the Binding to P-TEFb and Blocks the Expression of MHC Class II Genes in HIV Infection. Immunity 2000;12:61-70.

17. Mudhasani R, Fontes JD. The class II transactivator requires brahma-related gene 1 to activate transcription of major histocompatibility complex class II genes. Mol Cell Biol 2002;22:5019-26.

18. Zika E, Ting JP-Y. Epigenetic control of MHC-II: interplay between CIITA and histone-modifying enzymes. Curr Opin Immunol 2005;17:58-64.

19. Raval A, Howcroft TK, Weissman JD, Kirshner S, Zhu XS, Yokoyama K, et al. Transcriptional coactivator, CIITA, is an acetyltransferase that bypasses a promoter requirement for TAF(II)250. Mol Cell 2001;7:105-15.

20. Muhlethaler-Mottet A, Otten LA, Steimle V, Mach B. Expression of MHC class II molecules in different cellular and functional compartments is controlled by differential usage of multiple promoters of the transactivator CIITA. EMBO J 1997; 16:2851-60.

21. Landmann S, Muhlethaler-Mottet A, Bernasconi L, Suter T, Waldburger JM, Masternak K, et al. Maturation of dendritic cells is accompanied by rapid transcriptional silencing of class II transactivator (CIITA) expression. $J$ Exp Med 2001;194:379-91.

22. Cressman DE, O'Connor WJ, Greer SF, Zhu XS, Ting J. Mechanisms of nuclear import and export that control the subcellular localization of class II transactivator. J Immunol 2001;167:3626-34.

23. Barbieri G. Isoforms of the class II transactivator protein. Int Immunol 2002;14:839-48.

24. Nickerson K, Sisk TJ, Inohara N, Yee CS, Kennell J, Cho MC, et al. Dendritic 
cell-specific MHC class II transactivator contains a caspase recruitment domain that confers potent transactivation activity. J Biol Chem 2001;276:19089-93.

25. MartinezSoria E, Siegrist CA, Mach B. Highly efficient peptide binding and T cell activation by MHC class II molecules of CIITA-transfected cells. Int Immunol 1996;8:543-9.

26. Siegrist CA, Martinez-Soria E, Kern I, Mach B. A novel antigen-processingdefective phenotype in major histocompatibility complex class II-positive CIITA transfectants is corrected by interferon-gamma. $J$ Exp Med 1995;182:1793-9.

27. Chang $\mathrm{CH}$, Flavell RA. Class II transactivator regulates the expression of multiple genes involved in antigen presentation. $J$ Exp Med 1995;181:765-7.

28. Meazza R, Comes A, Orengo AM, Ferrini S, Accolla RS. Tumor rejection by gene transfer of the MHC class II transactivator in murine mammary adenocarcinoma cells. Eur J Immunol 2003;33:1183-92.

29. Hake SB, Masternak K, Kammerbauer C, Janzen C, Reith W, Steimle V. CIITA leucine-rich repeats control nuclear localization, in vivo recruitment to the major histocompatibility complex (MHC) class II enhanceosome, and MHC class II gene transactivation. Mol Cell Biol 2000;20:7716-25.

30. Kwon M-J, Soh J-W, Chang C-H. Protein kinase C delta is essential to maintain CIITA gene expression in B cells. J Immunol 2006;177:950-6.

31. Sisk TJ, Nickerson K, Kwok RPS, Chang C-H. Phosphorylation of class II transactivator regulates its interaction ability and transactivation function. Int Immunol 2003;15:1195-205.

32. Sisk TJ, Roys S, Chang CH. Self-association of CIITA and its transactivation potential. Mol Cell Biol 2001;21:4919-28.

33. Tosi G, Jabrane-Ferrat N, Peterlin BM. Phosphorylation of CIITA directs its oligomerization, accumulation and increased activity on MHCII promoters. EMBO J 2002;21:5467-76.

34. Scharer CD, Choi NM, Barwick BG, Majumder P, Lohsen S, Boss JM. Genome-wide CIITA-binding profile identifies sequence preferences that dictate function versus recruitment. Nucl Acids Res 2015;43:3128-42.

35. LeibundGut-Landmann S, Waldburger J-M, Krawczyk M, Otten LA, Suter T, Fontana A, et al. Mini-review: Specificity and expression of CIITA, the master regulator of MHC class II genes. Eur J Immunol 2004;34:1513-25.

36. Chin KC, Li GG, Ting JP. Importance of acidic, proline/serine/threonine-rich, and GTP-binding regions in the major histocompatibility complex class II transactivator: generation of transdominant-negative mutants. Proc Natl Acad Sci USA 1997;94:2501-6. 
37. Linhoff MW, Harton JA, Cressman DE, Martin BK, Ting JP. Two distinct domains within CIITA mediate self-association: involvement of the GTPbinding and leucine-rich repeat domains. Mol Cell Biol 2001;21:3001-11.

38. Harton JA, Ting JP. Class II transactivator: mastering the art of major histocompatibility complex expression. Mol Cell Biol 2000;20:6185-94.

39. Raval A, Weissman JD, Howcroft TK, Singer DS. The GTP-binding domain of class II transactivator regulates its nuclear export. J Immunol 2003;170:922-30.

40. Soe KC, Devaiah BN, Singer DS. Transcriptional coactivator CIITA, a functional homolog of TAF1, has kinase activity. Biochim Biophys Acta 2013;1829:1184-90.

41. Roeder RG. The role of general initiation factors in transcription by RNA polymerase II. Trends Biochem Sci 1996;21:327-35.

42. Devaiah BN, Lu H, Gegonne A, Sercan Z, Zhang H, Clifford RJ, et al. Novel Functions for TAF7, a Regulator of TAF1-independent Transcription. J Biol Chem 2010;285:38772-80.

43. Mizzen CA, Yang XJ, Kokubo T, Brownell JE, Bannister AJ, OwenHughes T, et al. The TAF(II)250 subunit of TFIID has histone acetyltransferase activity. Cell 1996;87:1261-70.

44. Gegonne A, Weissman JD, Singer DS. TAFII55 binding to TAFII250 inhibits its acetyltransferase activity. Proc Natl Acad Sci USA 2001;98:12432-7.

45. Dikstein R, Ruppert S, Tjian R. TAFII250 is a bipartite protein kinase that phosphorylates the base transcription factor RAP74. Cell 1996;84:781-90.

46. Gegonne A, Weissman JD, Zhou MS, Brady JN, Singer DS. TAF7: A possible transcription initiation check-point regulator. Proc Natl Acad Sci USA 2006;103:602-7.

47. Devaiah BN, Singer DS. CIITA and Its Dual Roles in MHC Gene Transcription. Front Immunol 2013;4:476.

48. Nagarajan UM, Bushey A, Boss JM. Modulation of gene expression by the MHC class II transactivator. J Immunol 2002;169:5078-88.

49. Wong D, Lee W, Humburg P, Makino S, Lau E. Genomic mapping of the MHC transactivator CIITA using an integrated ChIP-seq and genetical genomics approach. Genome Biol 2014;15:494.

50. Wong AW, Brickey WJ, Taxman DJ, van Deventer HW, Reed W, Gao JX, et al. CIITA-regulated plexin-A1 affects T-cell-dendritic cell interactions. Nat Immunol 2003;4:891-8.

51. Yee CSK, Yao Y, Xu Q, McCarthy B, Sun-Lin D, Tone M, et al. Enhanced production of IL-10 by dendritic cells deficient in CIITA. J Immunol 
$2005 ; 174: 1222-9$.

52. Kapsenberg ML. Dendritic-cell control of pathogen-driven T-cell polarization. Nat Rev Immunol 2003;3:984-93.

53. Gourley TS, Chang $\mathrm{CH}$. Cutting edge: the class II transactivator prevents activation-induced cell death by inhibiting Fas ligand gene expression. $J$ Immunol 2001;166:2917-21.

54. Owen-Schaub LB, Yonehara S, Crump WL, Grimm EA. DNA fragmentation and cell death is selectively triggered in activated human lymphocytes by Fas antigen engagement. Cell Immunol 1992;140:197-205.

55. Sisk TJ, Gourley T, Roys S, Chang CH. MHC class II transactivator inhibits IL-4 gene transcription by competing with NF-AT to bind the coactivator CREB binding protein (CBP)/p300. J Immunol 2000;165:2511-7.

56. Pai RK, Askew D, Boom WH, Harding CV. Regulation of class II MHC expression in APCs: Roles of types I, III, and IV class II transactivator. $J$ Immunol 2002;169:1326-33.

57. Piskurich JF, Linhoff MW, Wang Y, Ting JP. Two distinct gamma interferoninducible promoters of the major histocompatibility complex class II transactivator gene are differentially regulated by STAT1, interferon regulatory factor 1, and transforming growth factor beta. Mol Cell Biol 1999;19:431-40.

58. LeibundGut-Landmann S, Waldburger J-M, Reis e Sousa C, Acha-Orbea H, Reith W. MHC class II expression is differentially regulated in plasmacytoid and conventional dendritic cells. Nat Immunol 2004;5:899-908.

59. Waldburger JM, Suter T, Fontana A, Acha-Orbea H, Reith W. Selective abrogation of major histocompatibility complex class II expression on extrahematopoietic cells in mice lacking promoter IV of the class II transactivator gene. J Exp Med 2001;194:393-406.

60. Lennon AM, Ottone C, Rigaud G, Deaven LL, Longmire J, Fellous M, et al. Isolation of a B-cell-specific promoter for the human class II transactivator. Immunogenetics 1997;45:266-73.

61. Holling TM, van der Stoep N, Quinten E, van den Elsen PJ. Activated human T cells accomplish MHC class II expression through T cell-specific occupation of class II transactivator promoter III. J Immunol 2002;168:763-70.

62. Wong AW, Ghosh N, McKinnon KP, Reed W, Piskurich JF, Wright KL, et al. Regulation and specificity of MHC2TA promoter usage in human primary $\mathrm{T}$ lymphocytes and cell line. J Immunol 2002;169:3112-9.

63. Dong Y, Rohn WM, Benveniste EN. IFN-gamma regulation of the type IV class II transactivator promoter in astrocytes. J Immunol 1999;162:4731-9.

64. Muhlethaler-Mottet A, Di Berardino W, Otten LA, Mach B. Activation of the 
MHC class II transactivator CIITA by interferon-gamma requires cooperative interaction between Stat1 and USF-1. Immunity 1998;8:157-66.

65. Morris AC, Beresford GW, Mooney MR, Boss JM. Kinetics of a gamma interferon response: expression and assembly of CIITA promoter IV and inhibition by methylation. Mol Cell Biol 2002;22:4781-91.

66. Pattenden SG, Klose R, Karaskov E, Bremner R. Interferon-gamma-induced chromatin remodeling at the CIITA locus is BRG1 dependent. EMBO J 2002;21:1978-86.

67. Morris AC, Spangler WE, Boss JM. Methylation of Class II trans-Activator Promoter IV: A Novel Mechanism of MHC Class II Gene Control. J Immunol 2000;164:4143-9.

68. van den Elsen PJ, van der Stoep N, Viëtor HE, Wilson L, van Zutphen M, Gobin SJ. Lack of CIITA expression is central to the absence of antigen presentation functions of trophoblast cells and is caused by methylation of the IFN-gamma inducible promoter (PIV) of CIITA. Hum Immunol 2000;61:85062.

69. van der Stoep N, Biesta P, Quinten E, van den Elsen PJ. Lack of IFN-gammamediated induction of the class II transactivator (CIITA) through promoter methylation is predominantly found in developmental tumor cell lines. Int $J$ Cancer 2002;97:501-7.

70. Seguín-Estévez Q, Dunand-Sauthier I, Lemeille S, Iseli C, Ibberson M, Ioannidis $\mathrm{V}$, et al. Extensive remodeling of DC function by rapid maturationinduced transcriptional silencing. Nucl Acids Res 2014;42:9641-55.

71. De Lerma Barbaro A, Procopio FA, Mortara L, Tosi G, Accolla RS. The MHC class II transactivator (CIITA) mRNA stability is critical for the HLA class II gene expression in myelomonocytic cells. Eur J Immunol 2005;35:603-11.

72. Asirvatham AJ, Gregorie CJ, Hu Z, Magner WJ, Tomasi TB. MicroRNA targets in immune genes and the Dicer/Argonaute and ARE machinery components. Mol Immunol 2008;45:1995-2006.

73. Schnappauf F, Hake SB, Carvajal MMC, Bontron S, Lisowska Grospierre B, Steimle V. N-terminal destruction signals lead to rapid degradation of the major histocompatibility complex class II transactivator CIITA. Eur J Immunol 2003;33:2337-47.

74. Baumeister W, Walz J, Zühl F, Seemüller E. The proteasome: paradigm of a self-compartmentalizing protease. Cell 1998;92:367-80.

75. Coux O, Tanaka K, Goldberg AL. Structure and functions of the 20S and 26S proteasomes. Annu Rev Biochem 1996;65:801-47.

76. Strickland E, Hakala K, Thomas PJ, DeMartino GN. Recognition of misfolding proteins by PA700, the regulatory subcomplex of the $26 \mathrm{~S}$ proteasome. J Biol 
Chem 2000;275:5565-72.

77. Bhat KP, Truax AD, Greer SF. Phosphorylation and ubiquitination of degron proximal residues are essential for class II transactivator (CIITA) transactivation and major histocompatibility class II expression. $J$ Biol Chem 2010;285:25893-903.

78. Morgan JE, Shanderson RL, Boyd NH, Cacan E, Greer SF. The Class II Transactivator (CIITA) is regulated by Posttranslational modification crosstalk between ERK1/2 phosphorylation, mono-ubiquitination, and Lysine (K) 63 ubiquitination. Biosci Rep 2015;35:e0233.

79. Kong $\mathrm{X}$, Fang $\mathrm{M}$, Li $\mathrm{P}$, Fang $\mathrm{F}, \mathrm{Xu}$ Y. HDAC2 deacetylates class II transactivator and suppresses its activity in macrophages and smooth muscle cells. J Mol Cell Cardiol 2009;46:292-9.

80. Wu X, Kong X, Chen D, Li H, Zhao Y, Xia M, et al. SIRT1 links CIITA deacetylation to MHC II activation. Nucl Acids Res 2011;39:9549-58.

81. Bhat KP, Truax AD, Brooks JK, Greer SF. Association of the 19S proteasomal ATPases with the ATPase-binding domain of CIITA is essential for CIITA stability and MHC class II expression. Immunol Cell Biol 2010;88:807-16.

82. Ulbricht T, Alzrigat M, Horch A, Reuter N, Mikecz von A, Steimle V, et al. PML promotes MHC class II gene expression by stabilizing the class II transactivator. J Cell Biol 2012;199:49-63.

83. Cressman DE, Chin K-C, Taxman DJ, Ting JP-Y. A Defect in the Nuclear Translocation of CIITA Causes a Form of Type II Bare Lymphocyte Syndrome. Immunity 1999;10:163-71.

84. Spilianakis C, Papamatheakis J, Kretsovali A. Acetylation by PCAF enhances CIITA nuclear accumulation and transactivation of major histocompatibility complex class II genes. Mol Cell Biol 2000;20:8489-98.

85. Greer SF, Zika E, Conti B, Zhu X-S, Ting JP-Y. Enhancement of CIITA transcriptional function by ubiquitin. EMBO J 2003;4:1074-82.

86. Herrmann TL, Agrawal RS, Connolly SF, McCaffrey RL, Schlomann J, Kusner DJ. MHC Class II levels and intracellular localization in human dendritic cells are regulated by calmodulin kinase II. J Leukoc Biol 2007;82:686-99.

87. Piskurich JF, Lin KI, Lin Y, Wang Y, Ting JP, Calame K. BLIMP-I mediates extinction of major histocompatibility class II transactivator expression in plasma cells. Nat Immunol 2000;1:526-32.

88. Ghosh N, Gyory I, Wright G, Wood J, Wright KL. Positive regulatory domain I binding factor 1 silences class II transactivator expression in multiple myeloma cells. J Biol Chem 2001;276:15264-8.

89. Turley SJ, Inaba K, Garrett WS, Ebersold M, Unternaehrer J, Steinman RM, et 
al. Transport of peptide-MHC class II complexes in developing dendritic cells. Science 2000;288:522-7.

90. De Gassart A, Camosseto V, Thibodeau J, Ceppi M, Catalan N, Pierre P, et al. MHC class II stabilization at the surface of human dendritic cells is the result of maturation-dependent MARCH I down-regulation. PNAS 2008;105:3491-6.

91. Piskurich JF, Wang Y, Linhoff MW, White LC, Ting JP. Identification of distinct regions of $5^{\prime}$ flanking DNA that mediate constitutive, IFN-gamma, STAT1, and TGF-beta-regulated expression of the class II transactivator gene. J Immunol 1998;160:233-40.

92. Rohn W, Tang LP, Dong Y, Benveniste EN. IL-1 beta inhibits IFN-gammainduced class II MHC expression by suppressing transcription of the class II transactivator gene. J Immunol 1999;162:886-96.

93. O'Keefe GM, Nguyen VT, Ping Tang LL, Benveniste EN. IFN-gamma regulation of class II transactivator promoter IV in macrophages and microglia: involvement of the suppressors of cytokine signaling-1 protein. $J$ Immunol 2001;166:2260-9.

94. Trinchieri G, Perussia B. Immune interferon: a pleiotropic lymphokine with multiple effects. Immunol Today 1985;6:131-6.

95. Panelli MC, Wang E, Shen S, Schluter SF, Bernstein RM, Hersh EM, et al. Interferon gamma (IFNgamma) gene transfer of an EMT6 tumor that is poorly responsive to IFNgamma stimulation: increase in tumor immunogenicity is accompanied by induction of a mouse class II transactivator and class II MHC. Cancer Immunol Immunother 1996;42:99-107.

96. Krief PB, Azzarone B, Boucheix C, Billard C, Chang C, Fiers W, et al. Different levels of interferon- $\gamma$ induction of class I and class II major histocompatibility antigens in human hepatoma cell lines. In: Thiollais P, editors. Viral Hepatitis and Liver Disease New York: A. R. Liss, Inc.; 1988. p. 217.

97. Sartoris S, Valle MT, Barbaro AD, Tosi G, Cestari T, D'Agostino A, et al. HLA class II expression in uninducible hepatocarcinoma cells after transfection of AIR-1 gene product CIITA: Acquisition of antigen processing and presentation capacity. J Immunol 1998;161:814-20.

98. Satoh A, Toyota M, Ikeda H, Morimoto Y, Akino K, Mita H, et al. Epigenetic inactivation of class II transactivator (CIITA) is associated with the absence of interferon- $\gamma$-induced HLA-DR expression in colorectal and gastric cancer cells. Oncogene 2004;23:8876-86.

99. Chang CH, Fontes JD, Peterlin M, Flavell RA. Class II transactivator (CIITA) is sufficient for the inducible expression of major histocompatibility complex class II genes. J Exp Med 1994;180:1367-74.

100. Steimle V, Siegrist CA, Mottet A, Lisowska-Grospierre B, Mach B. Regulation 
of MHC class II expression by interferon-gamma mediated by the transactivator gene CIITA. Science 1994;265:106-9.

101. Chin KC, Mao C, Skinner C, Riley JL, Wright KL, Moreno CS, et al. Molecular analysis of G1B and G3A IFN gamma mutants reveals that defects in CIITA or RFX result in defective class II MHC and Ii gene induction. Immunity 1994;1:687-97.

102. Thompson JA, Dissanayake SK, Ksander BR, Knutson KL, Disis ML, OstrandRosenberg S. Tumor cells transduced with the MHC class II Transactivator and CD80 activate tumor-specific CD4+ T cells whether or not they are silenced for invariant chain. Cancer Res 2006;66:1147-54.

103. Martin BK, Frelinger JG, Ting JP. Combination gene therapy with CD86 and the MHC class II transactivator in the control of lung tumor growth. $J$ Immunol 1999; 162:6663-70.

104. Lu X, Kallinteris NL, Li J, Wu S, Li Y, Jiang Z, et al. Tumor immunotherapy by converting tumor cells to MHC class II-positive, Ii protein-negative phenotype. Cancer Immunol Immunother 2003;52:592-8.

105. Wang Y, Xu M, Che M, Hofe Von E, Abbas A, Kallinteris NL, et al. Curative antitumor immune response is optimal with tumor irradiation followed by genetic induction of major histocompatibility complex class I and class II molecules and suppression of Ii protein. Hum Gene Ther 2005;16:187-99.

106. Mortara L, Castellani P, Meazza R, Tosi G, De Lerma Barbaro A, Procopio FA, et al. CIITA-induced MHC class II expression in mammary adenocarcinoma leads to a Th1 polarization of the tumor microenvironment, tumor rejection, and specific antitumor memory. Clin Cancer Res 2006;12:3435-43.

107. Mortara L, Frangione V, Castellani P, De Lerma Barbaro A, Accolla RS. Irradiated CIITA-positive mammary adenocarcinoma cells act as a potent antitumor-preventive vaccine by inducing tumor-specific CD4+ T cell priming and CD8+ T cell effector functions. Int Immunol 2009;21:655-65.

108. Frangione V, Mortara L, Castellani P, De Lerma Barbaro A, Accolla RS. CIITA-driven MHC-II positive tumor cells: Preventive vaccines and superior generators of antitumor CD4+ T lymphocytes for immunotherapy. Int J Cancer 2010;127:1614-24.

109. Stumptner-Cuvelette $\mathrm{P}$, Benaroch P. Multiple roles of the invariant chain in MHC class II function. BBA-Mol Cell Res 2002;1542:1-13.

110. Banchereau J, Briere F, Caux C, Davoust J, Lebecque S, Liu YJ, et al. Immunobiology of dendritic cells. Annu Rev Immunol 2000;18:767-811.

111. Steinman RM, Banchereau J. Taking dendritic cells into medicine. Nature 2007;449:419-26.

112. Banchereau J, Steinman RM. Dendritic cells and the control of immunity. 
Nature 1998;392:245-52.

113. Steinman RM. The control of immunity and tolerance by dendritic cells. Pathol Biol 2003;51:59-60.

114. Ridgway D. The first 1000 dendritic cell vaccinees. Cancer Invest 2003;21:873-86.

115. Lu W, Arraes LC, Ferreira WT, Andrieu J-M. Therapeutic dendritic-cell vaccine for chronic HIV-1 infection. Nat Med 2004;10:1359-65.

116. García F, Lejeune M, Climent N, Gil C, Alcamí J, Morente V, et al. Therapeutic immunization with dendritic cells loaded with heat-inactivated autologous HIV-1 in patients with chronic HIV-1 infection. J Infect Dis 2005;191:1680-5.

117. Hansen M, Hjortø GM, Donia M, Met Ö, Larsen NB, Andersen MH, et al. Comparison of clinical grade type 1 polarized and standard matured dendritic cells for cancer immunotherapy. Vaccine 2013;31:639-46.

118. Kalinski P, Moser M. Consensual immunity: success-driven development of Thelper-1 and T-helper-2 responses. Nat Rev Immunol 2005;5:251-60.

119. Hansen M, Hjortø GM, Donia M, Met Ö, Larsen NB. Comparison of clinical grade type 1 polarized and standard matured dendritic cells for cancer immunotherapy. Vaccine 2013;31:639-46.

120. Tanaka H, Demeure CE, Rubio M, Delespesse G, Sarfati M. Human monocytederived dendritic cells induce naive $\mathrm{T}$ cell differentiation into $\mathrm{T}$ helper cell type 2 (Th2) or Th1/Th2 effectors. Role of stimulator/responder ratio. J Exp Med 2000;192:405-12.

121. Moser M, Murphy KM. Dendritic cell regulation of TH1-TH2 development. Nat Immunol 2000;1:199-205.

122. Kalinski P, Schuitemaker JH, Hilkens CM, Kapsenberg ML. Prostaglandin E2 induces the final maturation of IL-12-deficient CD1a+CD83+ dendritic cells: the levels of IL-12 are determined during the final dendritic cell maturation and are resistant to further modulation. J Immunol 1998;161:2804-9.

123. Kalinski P, Schuitemaker JH, Hilkens CM, Wierenga EA, Kapsenberg ML. Final maturation of dendritic cells is associated with impaired responsiveness to IFN-gamma and to bacterial IL-12 inducers: decreased ability of mature dendritic cells to produce IL-12 during the interaction with Th cells. J Immunol 1999;162:3231-6.

124. de Jong EC, Vieira PL, Kalinski P, Schuitemaker JHN, Tanaka Y, Wierenga EA, et al. Microbial compounds selectively induce Th1 cell-promoting or Th2 cell-promoting dendritic cells in vitro with diverse th cell-polarizing signals. $J$ Immunol 2002;168:1704-9. 
125. Gagliardi MC, Sallusto F, Marinaro M, Langenkamp A, Lanzavecchia A, De Magistris MT. Cholera toxin induces maturation of human dendritic cells and licences them for Th2 priming. Eur J Immunol 2000;30:2394-403.

126. Vieira PL, de Jong EC, Wierenga EA, Kapsenberg ML, Kalinski P. Development of Th1-inducing capacity in myeloid dendritic cells requires environmental instruction. $J$ Immunol 2000;164:4507-12.

127. Mailliard RB, Egawa S, Cai Q, Kalinska A, Bykovskaya SN, Lotze MT, et al. Complementary dendritic cell-activating function of $\mathrm{CD} 8(+)$ and $\mathrm{CD} 4(+) \mathrm{T}$ cells: Helper role of CD8(+) T cells in the development of T helper type 1 responses. $J$ Exp Med 2002;195:473-83.

128. Mailliard RB, Son YI, Redlinger R, Coates PT, Giermasz A, Morel PA, et al. Dendritic cells mediate NK cell help for Th1 and CTL responses: Two-signal requirement for the induction of $\mathrm{NK}$ cell helper function. $J$ Immunol 2003;171:2366-73.

129. Mailliard RB, Wankowicz-Kalinska A, Cai Q, Wesa A, Hilkens CM, Kapsenberg ML, et al. alpha-type-1 polarized dendritic cells: A novel immunization tool with optimized CTL-inducing activity. Cancer Res 2004;64:5934-7.

130. López-Albaitero A, Mailliard R, Hackman T, Andrade Filho PA, Wang X, Gooding W, et al. Maturation pathways of dendritic cells determine TAP1 and TAP2 levels and cross-presenting function. J Immunother 2009;32:465-73.

131. Jeras M, Bergant M, Repnik U. In vitro preparation and functional assessment of human monocyte-derived dendritic cells-potential antigen-specific modulators of in vivo immune responses. Transpl Immunol 2005;14:231-44.

132. Jonuleit H, Kühn U, Müller G, Steinbrink K, Paragnik L, Schmitt E, et al. Proinflammatory cytokines and prostaglandins induce maturation of potent immunostimulatory dendritic cells under fetal calf serum-free conditions. Eur $J$ Immunol 1997;27:3135-42.

133. O'Garra A. Cytokines induce the development of functionally heterogeneous $\mathrm{T}$ helper cell subsets. Immunity 1998;8:275-83.

134. Watchmaker PB, Berk E, Muthuswamy R, Mailliard RB, Urban JA, Kirkwood $\mathrm{JM}$, et al. Independent regulation of chemokine responsiveness and cytolytic function versus $\mathrm{CD} 8+\mathrm{T}$ cell expansion by dendritic cells. J Immunol 2010;184:591-7.

135. Su Z, Dannull J, Yang BK, Dahm P, Coleman D, Yancey D, et al. Telomerase mRNA-transfected dendritic cells stimulate antigen-specific CD8(+) and CD4(+) T cell responses in patients with metastatic prostate cancer. $J$ Immunol 2005; 174:3798-807.

136. Calderhead DM, DeBenedette MA, Ketteringham H, Gamble AH, Horvatinovich JM, Tcherepanova IY, et al. Cytokine maturation followed by 
CD40L mRNA electroporation results in a clinically relevant dendritic cell product capable of inducing a potent proinflammatory CTL response. $J$ Immunother 2008;31:731-41.

137. Wolfraim LA, Takahara M, Viley AM, Shivakumar R, Nieda M, Maekawa R, et al. Clinical scale electroloading of mature dendritic cells with melanoma whole tumor cell lysate is superior to conventional lysate co-incubation in triggering robust in vitro expansion of functional antigen-specific CTL. Int Immunopharmacol 2013;15:488-97.

138. Fairman P, Angel JB. The effect of human immunodeficiency virus-1 on monocyte-derived dendritic cell maturation and function. Clin Exp Immunol 2012;170:101-13.

139. Routy J-P, Boulassel M-R, Yassine-Diab B, Nicolette C, Healey D, Jain R, et al. Immunologic activity and safety of autologous HIV RNA-electroporated dendritic cells in HIV-1 infected patients receiving antiretroviral therapy. Clin Immunol 2010;134:140-7.

140. Allard SD, De Keersmaecker B, de Goede AL, Verschuren EJ, Koetsveld J, Reedijk ML, et al. A phase I/IIa immunotherapy trial of HIV-1-infected patients with Tat, Rev and Nef expressing dendritic cells followed by treatment interruption. Clin Immunol 2012;142:252-68.

141. Huang X-L, Fan Z, Borowski L, Rinaldo CR. Multiple T-Cell Responses to Human Immunodeficiency Virus Type 1 Are Enhanced by Dendritic Cells. Clin Vaccine Immunol 2009;16:1504-16.

142. Niu L, Termini JM, Kanagavelu SK, Gupta S, Rolland MM, Kulkarni V, et al. Preclinical evaluation of HIV-1 therapeutic ex vivo dendritic cell vaccines expressing consensus Gag antigens and conserved Gag epitopes. Vaccine 2011;29:2110-9.

143. Connolly NC, Whiteside TL, Wilson C, Kondragunta V, Rinaldo CR, Riddler SA. Therapeutic immunization with human immunodeficiency virus type 1 (HIV-1) peptide-loaded dendritic cells is safe and induces immunogenicity in HIV-1-infected individuals. Clin Vaccine Immunol 2008;15:284-92.

144. Huang X-L, Fan Z, Borowski L, Rinaldo CR. Maturation of dendritic cells for enhanced activation of anti-HIV-1 CD8(+) T cell immunity. J Leukoc Biol 2008;83:1530-40.

145. Trepiakas R, Pedersen AE, Met Ö, Hansen MH. Comparison of $\alpha$-Type-1 polarizing and standard dendritic cell cytokine cocktail for maturation of therapeutic monocyte-derived dendritic cell preparations from cancer patients. Vaccine 2008;26:2824-32.

146. Kalinski P, Nakamura Y, Watchmaker P, Giermasz A, Muthuswamy R, Mailliard RB. Helper roles of NK and CD8(+) T cells in the induction of tumor immunity - Polarized dendritic cells as cancer vaccines. Immunol Res 
2006;36:137-46.

147. Grünebach F, Erndt S, Häntschel M, Heine A, Brossart P. Generation of antigen-specific CTL responses using RGS1 mRNA transfected dendritic cells. Cancer Immunol Immunother 2008;57:1483-91.

148. Barbuto JAM. Are dysfunctional monocyte-derived dendritic cells in cancer an explanation for cancer vaccine failures? Immunotherapy 2013;5:105-7.

149. Ramos RN, Chin LS, Santos Dos APSA, Bergami-Santos PC, Laginha F, Barbuto JAM. Monocyte-derived dendritic cells from breast cancer patients are biased to induce CD4+CD25+Foxp3+ regulatory T cells. J Leukoc Biol 2012;92:673-82.

150. Fantuzzi L, Purificato C, Donato K, Belardelli F, Gessani S. Human Immunodeficiency Virus Type 1 gp120 Induces Abnormal Maturation and Functional Alterations of Dendritic Cells: a Novel Mechanism for AIDS Pathogenesis. J Virol 2004;78:9763-72.

151. Shan M, Klasse PJ, Banerjee K, Dey AK, Iyer SPN, Dionisio R, et al. HIV-1 gp120 Mannoses Induce Immunosuppressive Responses from Dendritic Cells. PLoS Pathog 2007;3:e169.

152. Connolly N, Riddler S, Stanson J, Gooding W, Rinaldo CR, Ferrone S, et al. Levels of antigen processing machinery components in dendritic cells generated for vaccination of HIV-1+ subjects. AIDS 2007;21:1683-92.

153. Minskaia E, Ryan MD. Protein Coexpression Using FMDV 2A: Effect of "Linker" Residues. Biomed Res Int 2013;2013:-12.

154. Weber K, Bartsch U, Stocking C, Fehse B. A Multicolor Panel of Novel Lentiviral "Gene Ontology" (LeGO) Vectors for Functional Gene Analysis. Mol Ther 2008;16:698-706.

155. Kavanagh DG. Expansion of HIV-specific CD4+ and CD8+ $\mathrm{T}$ cells by dendritic cells transfected with mRNA encoding cytoplasm- or lysosometargeted Nef. Blood 2006;107:1963-9.

156. Beyer WR, Westphal M, Ostertag W, Laer von D. Oncoretrovirus and lentivirus vectors pseudotyped with lymphocytic choriomeningitis virus glycoprotein: generation, concentration, and broad host range. J Virol 2002;76:1488-95.

157. Douradinha B, McBurney SP, Soares de Melo KM, Smith AP, Krishna NK, Barratt-Boyes SM, et al. C1q binding to dengue virus decreases levels of infection and inflammatory molecules transcription in THP-1 cells. Virus Res 2014;179:231-4.

158. Toniolo PA, Liu S, Yeh JE, Moraes-Vieira PM, Walker SR, Vafaizadeh V, et al. Inhibiting STAT5 by the BET bromodomain inhibitor JQ1 disrupts human dendritic cell maturation. J Immunol 2015;194:3180-90. 
159. Livak KJ, Schmittgen TD. Analysis of Relative Gene Expression Data Using Real-Time Quantitative PCR and the 2- $\Delta \Delta$ CT Method. Methods 2001;25:4028.

160. Lohsen S, Majumder P, Scharer CD, Barwick BG, Austin JW, Zinzow-Kramer WM, et al. Common distal elements orchestrate CIITA isoform-specific expression in multiple cell types. Genes Immun 2014;15:543-55.

161. Xia J, Fang M, Wu X, Yang Y, Yu L, Xu H, et al. A2b adenosine signaling represses CIITA transcription via an epigenetic mechanism in vascular smooth muscle cells. Biochim Biophys Acta 2015;1849:665-76.

162. Schlahsa L, Zhang H, Battermann A, Verboom M, Immenschuh S, Eiz-Vesper $\mathrm{B}$, et al. Semaphorin 3A alters endothelial cell immunogenicity by regulating Class II transactivator activity circuits. Transfusion 2014;54:1961-70.

163. Wright KL, Ting JP-Y. Epigenetic regulation of MHC-II and CIITA genes. Trends Immunol 2006;27:405-12.

164. Green MR, Yoon H, Boss JM. Epigenetic Regulation during B Cell Differentiation Controls CIITA Promoter Accessibility. $J$ Immunol 2006; 177:3865-73.

165. Smith MA, Wright $\mathrm{G}$, Wu J, Tailor P, Ozato $\mathrm{K}$, Chen $\mathrm{X}$, et al. Positive regulatory domain I (PRDM1) and IRF8/PU.1 counter-regulate MHC class II transactivator (CIITA) expression during dendritic cell maturation. J Biol Chem 2011;286:7893-904.

166. Wu X, Kong X, Luchsinger L, Smith BD, Xu Y. Regulating the Activity of Class II Transactivator by Posttranslational Modifications: Exploring the Possibilities. Mol Cell Biol 2009;29:5639-44.

167. Donnelly M, Hughes LE, Luke G, Mendoza H, Dam ten E, Gani D, et al. The 'cleavage' activities of foot-and-mouth disease virus $2 \mathrm{~A}$ site-directed mutants and naturally occurring "2A-like" sequences. J Gen Virol 2001;82:1027-41.

168. Maciel MJ, Pereira Cruz FDS, Cordeiro MT, da Motta MA, Soares de Melo Cassemiro KM, Carvalho Maia $\mathrm{R}$ de $\mathrm{C}$, et al. A DNA Vaccine against Yellow Fever Virus: Development and Evaluation. PLoS Negl Trop Dis 2015;9.

169. Mah C, Fraites TJ, Cresawn KO, Zolotukhin I, Lewis MA, Byrne BJ. A new method for recombinant adeno-associated virus vector delivery to murine diaphragm. Mol Ther 2004;9:458-63.

170. Greer SF, Harton JA, Linhoff MW, Janczak CA, Ting J, Cressman DE. Serine residues 286, 288, and 293 within the CIITA: A mechanism for downregulating CIITA activity through phosphorylation. J Immunol 2004;173:37683.

171. Voong LN, Slater AR, Kratovac S, Cressman DE. Mitogen-activated protein kinase ERK1/2 regulates the class II transactivator. $J$ Biol Chem 
2008;283:9031-9.

172. Cressman DE, O'Connor WJ, Greer SF, Zhu XS, Ting JP. Mechanisms of nuclear import and export that control the subcellular localization of class II transactivator. J Immunol 2001;167:3626-34.

173. Barbaro ADL, Tosi G, Frumento G, Bruschi E, D'Agostino A, Valle MT, et al. Block of Stat-1 activation in macrophages phagocytosing bacteria causes reduced transcription of CIITA and consequent impaired antigen presentation. Eur J Immunol 2002;32:1309-18.

174. Chiu E, Gold T, Fettig V, LeVasseur MT, Cressman DE. Identification of a Nuclear Export Sequence in the MHC CIITA. J Immunol 2015;194:6102-11.

175. Camacho-Carvajal MM, Klingler S, Schnappauf F, Hake SB, Steimle V. Importance of class II transactivator leucine-rich repeats for dominant-negative function and nucleo-cytoplasmic transport. Int Immunol 2004;16:65-75.

176. Qing G, Yan P, Xiao G. Hsp90 inhibition results in autophagy-mediated proteasome-independent degradation of IkappaB kinase (IKK). Cell Res 2006;16:895-901.

177. Tao T, Shi H, Guan Y, Huang D, Chen Y, Lane DP, et al. Def defines a conserved nucleolar pathway that leads p53 to proteasome-independent degradation. Cell Res 2013;23:620-34.

178. Condemine W, Takahashi Y, Zhu J, Puvion-Dutilleul F, Guegan S, Janin A, et al. Characterization of endogenous human promyelocytic leukemia isoforms. Cancer Res 2006;66:6192-8.

179. Jensen K, Shiels C, Freemont PS. PML protein isoforms and the RBCC/TRIM motif. Oncogene 2001;20:7223-33.

180. Ishov AM, Sotnikov AG, Negorev D, Vladimirova OV, Neff N, Kamitani T, et al. PML is critical for ND10 formation and recruits the PML-interacting protein daxx to this nuclear structure when modified by SUMO-1. J Cell Biol 1999;147:221-34.

181. Zhong S, Müller S, Ronchetti S, Freemont PS, Dejean A, Pandolfi PP. Role of SUMO-1-modified PML in nuclear body formation. Blood 2000;95:2748-52.

182. Zaccard CR, Watkins SC, Kalinski P, Fecek RJ, Yates AL, Salter RD, et al. $\mathrm{CD} 40 \mathrm{~L}$ induces functional tunneling nanotube networks exclusively in dendritic cells programmed by mediators of type 1 immunity. $J$ Immunol 2015;194:1047-56.

183. Landi A, Babiuk LA, Littel-van den Hurk SVD. High transfection efficiency, gene expression, and viability of monocyte-derived human dendritic cells after nonviral gene transfer. J Leukoc Biol 2007;82:849-60.

184. Li G-B, Lu G-X. Gene delivery efficiency in bone marrow-derived dendritic 
cells: comparison of four methods and optimization for lentivirus transduction. Mol Biotechnol 2009;43:250-6.

185. Breckpot K, Emeagi P, Dullaers M, Michiels A, Heirman C, Thielemans K. Activation of Immature Monocyte-Derived Dendritic Cells After Transduction with High Doses of Lentiviral Vectors. Hum Gene Ther 2007;18:536-46.

186. He Y, Zhang J, Mi Z, Robbins P, Falo LD. Immunization with lentiviral vectortransduced dendritic cells induces strong and long-lasting $\mathrm{T}$ cell responses and therapeutic immunity. J Immunol 2005;174:3808-17.

187. Croft M, Dubey C. Accessory molecule and costimulation requirements for CD4 T cell response. Crit Rev Immunol 1997;17:89-118.

188. Fan W, Tian X-D, Huang E, Zhang J-J. Exosomes from CIITA-Transfected CT26 Cells Enhance Anti-tumor Effects. Asian Pac $J$ Cancer Prev 2013;14:987-91.

189. Zhou X, Jiang Y, Lu L, Ding Q, Jiao Z, Zhou Y, et al. MHC class II transactivator represses human IL-4 gene transcription by interruption of promoter binding with $\mathrm{CBP} / \mathrm{p} 300$, STAT6 and NFAT1 via histone hypoacetylation. Immunology 2007;122:476-85.

190. Yee C, Yao Y, Li P, Klemsz MJ. Cathepsin E: a novel target for regulation by class II transactivator. J Immunol 2004;172:5528-34.

191. Gourley TS, Patel DR, Nickerson K, Hong S-C, Chang C-H. Aberrant expression of Fas ligand in mice deficient for the MHC class II transactivator. $J$ Immunol 2002;168:4414-9.

192. Zhong S, Salomoni P, Pandolfi PP. The transcriptional role of PML and the nuclear body. Nat Cell Biol 2000;2:E85-90.

193. Gamell C, Jan Paul P, Haupt Y, Haupt S. PML tumour suppression and beyond: therapeutic implications. FEBS Lett 2014;588:2653-62.

194. Duprez E, Saurin AJ, Desterro JM, Lallemand-Breitenbach V, Howe K, Boddy MN, et al. SUMO-1 modification of the acute promyelocytic leukaemia protein PML: implications for nuclear localisation. J Cell Sci 1999;112 ( Pt 3):381-93.

195. Kamitani T, Kito K, Nguyen HP, Wada H, Fukuda-Kamitani T, Yeh ETH. Identification of Three Major Sentrinization Sites in PML. J Biol Chem 1998;273:26675-82.

196. Lallemand-Breitenbach V, Zhu J, Puvion F, Koken M, Honoré N, Doubeikovsky A, et al. Role of promyelocytic leukemia (PML) sumolation in nuclear body formation, 11S proteasome recruitment, and As2O3-induced PML or PML/retinoic acid receptor alpha degradation. J Exp Med 2001;193:136171.

197. Sahin U, Lallemand-Breitenbach V, de Thé H. PML nuclear bodies: regulation, 
function and therapeutic perspectives. J Pathol 2014;234:289-91.

198. Gialitakis M, Arampatzi P, Makatounakis T, Papamatheakis J. Gamma interferon-dependent transcriptional memory via relocalization of a gene locus to PML nuclear bodies. Mol Cell Biol 2010;30:2046-56.

199. Bernardi R, Pandolfi PP. Structure, dynamics and functions of promyelocytic leukaemia nuclear bodies. Nat Rev Mol Cell Biol 2007;8:1006-16.

200. Jones DEJ, Palmer JM, Burt AD, Walker C, Robe AJ, Kirby JA. Bacterial motif DNA as an adjuvant for the breakdown of immune self-tolerance to pyruvate dehydrogenase complex. Hepatology 2002;36:679-86.

201. Djilali-Saiah I, Lapierre P, Vittozi S, Alvarez F. DNA vaccination breaks tolerance for a neo-self antigen in liver: a transgenic murine model of autoimmune hepatitis. J Immunol 2002;169:4889-96.

202. Ohta A, Sekimoto M, Sato M, Koda T, Nishimura S, Iwakura Y, et al. Indispensable role for TNF-alpha and IFN-gamma at the effector phase of liver injury mediated by Th1 cells specific to hepatitis B virus surface antigen. $J$ Immunol 2000;165:956-61.

203. Sacher T, Knolle P, Nichterlein T, Arnold B, Hämmerling GJ, Limmer A. CpGODN-induced inflammation is sufficient to cause T-cell-mediated autoaggression against hepatocytes. Eur J Immunol 2002;32:3628-37.

204. Friese MA, Jones EY, Fugger L. MHC II molecules in inflammatory diseases: interplay of qualities and quantities. Trends Immunol 2005;26:559-61.

205. Kim TW, Park HJ, Choi EY, Jung KC. Overexpression of CIITA in T cells aggravates Th2-mediated colitis in mice. J Korean Med Sci 2006;21:877-82.

206. Pennini ME, Pai RK, Schultz DC, Boom WH, Harding CV. Mycobacterium tuberculosis $19-\mathrm{kDa}$ lipoprotein inhibits IFN-gamma-induced chromatin remodeling of MHC2TA by TLR2 and MAPK signaling. $J$ Immunol 2006; 176:4323-30.

207. O'Keefe GM, Nguyen VT, Benveniste EN. Class II transactivator and class II MHC gene expression in microglia: modulation by the cytokines TGF-beta, IL4, IL-13 and IL-10. Eur J Immunol 1999;29:1275-85.

208. Nakamura MC. CIITA: a master regulator of adaptive immunity shows its innate side in the bone. J Bone Miner Res 2014;29:287-9.

209. Kim D, Hoory T, Monie A, Ting JP-Y, Hung C-F, Wu T-C. Enhancement of DNA vaccine potency through coadministration of CIITA DNA with DNA vaccines via gene gun. J Immunol 2008;180:7019-27.

210. Oshiro TM, de Almeida A, da Silva Duarte AJ. Dendritic cell immunotherapy for HIV infection: from theory to reality. Immunotherapy 2009;1:1039-51. 
211. Sato PK, Oshiro TM, Diogo CL, Passos EC, Shikanai-Yasuda MA. Characterization of monocyte-derived dendritic cells from patients with active and treated paracoccidioidomycosis. Scand J Immunol 2011;74:609-18.

212. Pontillo A, Silva LT, Oshiro TM, Finazzo C, Crovella S, Duarte AJS. HIV-1 induces NALP3-inflammasome expression and interleukin-1 $\beta$ secretion in dendritic cells from healthy individuals but not from HIV-positive patients. AIDS 2012;26:11-8.

213. Pontillo A, Santillo BT, Duarte AJ, Oshiro TM. Differential inflammasome expression and IL-1 $\beta$ secretion in monocyte-derived dendritic cells differentiated with IL-4 or IFN- $\alpha$. AIDS Res Ther 2013;10:35.

214. Irvine AS, Trinder PK, Laughton DL, Ketteringham H, McDermott RH, Reid $\mathrm{SC}$, et al. Efficient nonviral transfection of dendritic cells and their use for in vivo immunization. Nature Biotechnol 2000;18:1273-8.

215. Haines AM, Irvine AS, Mountain A, Charlesworth J, Farrow NA, Husain RD, et al. CL22 - a novel cationic peptide for efficient transfection of mammalian cells. Gene Ther 2001;8:99-110.

216. Lundqvist A, Noffz G, Pavlenko M, Sæbøe-Larssen S, Fong T, Maitland N, et al. Nonviral and Viral Gene Transfer Into Different Subsets of Human Dendritic Cells Yield Comparable Efficiency of Transfection. J Immunother 2002;25:445.

217. Chamarthy SP, Jia L, Kovacs JR, Anderson KR, Shen H, Firestine SM, et al. Gene delivery to dendritic cells facilitated by a tumor necrosis factor alphacompeting peptide. Mol Immunol 2004;41:741-9.

218. Tan PH, Beutelspacher SC, Wang Y-H, McClure MO, Ritter MA, Lombardi G, et al. Immunolipoplexes: an efficient, nonviral alternative for transfection of human dendritic cells with potential for clinical vaccination. Mol Ther 2005;11:790-800.

219. Lenz P, Bacot SM, Frazier-Jessen MR, Feldman GM. Nucleoporation of dendritic cells: efficient gene transfer by electroporation into human monocytederived dendritic cells. FEBS Lett 2003;538:149-54.

220. Erhardt M, Gorschlüter M, Sager J, Ziske C, Strehl J, Lilienfeld-Toal MV, et al. Transfection of human monocyte-derived dendritic cells with CpG oligonucleotides. Immunol Cell Biol 2005;83:278-85.

221. Van Tendeloo VF, Snoeck HW, Lardon F, Vanham GL, Nijs G, Lenjou M, et al. Nonviral transfection of distinct types of human dendritic cells: highefficiency gene transfer by electroporation into hematopoietic progenitor- but not monocyte-derived dendritic cells. Gene Ther 1998;5:700-7.

222. Arthur JF, Butterfield LH, Roth MD, Bui LA, Kiertscher SM, Lau R, et al. A comparison of gene transfer methods in human dendritic cells. Cancer Gene Ther 1997;4:17-25. 
223. Chen Y-Z, Ruan G-X, Yao X-L, Li L-M, Hu Y, Tabata Y, et al. Co-transfection Gene Delivery of Dendritic Cells Induced Effective Lymph Node Targeting and Anti-tumor Vaccination. Pharm Res 2013;30:1502-12.

224. Van Tendeloo VF, Ponsaerts P, Lardon F, Nijs G, Lenjou M, Van Broeckhoven $\mathrm{C}$, et al. Highly efficient gene delivery by mRNA electroporation in human hematopoietic cells: superiority to lipofection and passive pulsing of mRNA and to electroporation of plasmid cDNA for tumor antigen loading of dendritic cells. Blood 2001;98:49-56.

225. Mu LJ, Gaudernack G, Saeboe-Larssen S, Hammerstad H, Tierens A, Kvalheim G. A protocol for generation of clinical grade mRNA-transfected monocytederived dendritic cells for cancer vaccines. Scand J Immunol 2003;58:578-86.

226. Mu LJ, Kyte JA, Kvalheim G, Aamdal S, Dueland S, Hauser M, et al. Immunotherapy with allotumour mRNA-transfected dendritic cells in androgen-resistant prostate cancer patients. Br J Cancer 2005;93:749-56.

227. Michiels A, Tuyaerts S, Bonehill A, Corthals J, Breckpot K, Heirman C, et al. Electroporation of immature and mature dendritic cells: implications for dendritic cell-based vaccines. Gene Ther 2005;12:772-82.

228. Gholamin M, Moaven O, Farshchian M, Mahmoudi M, Sankian M, Memar B, et al. Induction of cytotoxic $\mathrm{T}$ lymphocytes primed with tumor RNA-loaded dendritic cells in esophageal squamous cell carcinoma: preliminary step for DC vaccine design. BMC Cancer 2010;10:261.

229. Morita Y, Gupta R, Seidl KM, McDonagh KT, Fox DA. Cytokine production by dendritic cells genetically engineered to express IL-4: induction of Th2 responses and differential regulation of IL-12 and IL-23 synthesis. $J$ Gene Med 2005;7:869-77.

230. Gruber A, Kan-Mitchell J, Kuhen KL, Mukai T. Dendritic cells transduced by multiply deleted HIV-1 vectors exhibit normal phenotypes and functions and elicit an HIV-specific cytotoxic T-lymphocyte response in vitro. Blood 2000;96:1327-33.

231. Nègre D, Mangeot PE, Duisit G, Blanchard S, Vidalain PO, Leissner P, et al. Characterization of novel safe lentiviral vectors derived from simian immunodeficiency virus (SIVmac251) that efficiently transduce mature human dendritic cells. Gene Ther 2000;7:1613-23.

232. Jonuleit H, Tüting T, Steitz J, Brück J, Giesecke A, Steinbrink K, et al. Efficient transduction of mature CD83+ dendritic cells using recombinant adenovirus suppressed T cell stimulatory capacity. Gene Ther 2000;7:249-54.

233. Schütz C, Hoves S, Halbritter D, Zhang H-G, Mountz JD, Fleck M. Alloantigen specific deletion of primary human $\mathrm{T}$ cells by Fas ligand (CD95L)-transduced monocyte-derived killer-dendritic cells. Immunology 2011;133:115-22.

234. Pecher G, Spahn G, Schirrmann T, Kulbe H, Ziegner M, Schenk JA, et al. 
Mucin gene (MUC1) transfer into human dendritic cells by cationic liposomes and recombinant adenovirus. Anticancer Res 2001;21:2591-6.

235. Esslinger C, Romero $\mathrm{P}$, MacDonald HR. Efficient transduction of dendritic cells and induction of a T-cell response by third-generation lentivectors. Hum Gene Ther 2002;13:1091-100.

236. Lizée G, Gonzales MI, Topalian SL. Lentivirus vector-mediated expression of tumor-associated epitopes by human antigen presenting cells. Hum Gene Ther 2004;15:393-404.

237. Lu D, Benjamin R, Kim M, Conry RM, Curiel DT. Optimization of methods to achieve mRNA-mediated transfection of tumor cells in vitro and in vivo employing cationic liposome vectors. Cancer Gene Ther 1994;1:245-52.

238. Ying H, Zaks TZ, Wang RF, Irvine KR, Kammula US, Marincola FM, et al. Cancer therapy using a self-replicating RNA vaccine. Nat Med 1999;5:823-7.

239. Chung DJ, Romano E, Pronschinske KB, Shyer JA, Mennecozzi M, St Angelo ET, et al. Langerhans-type and monocyte-derived human dendritic cells have different susceptibilities to mRNA electroporation with distinct effects on maturation and activation: implications for immunogenicity in dendritic cellbased immunotherapy. J Transl Med 2013;11:166.

240. Bikard D, Loot C, Baharoglu Z, Mazel D. Folded DNA in action: hairpin formation and biological functions in prokaryotes. Microbiol Mol Biol Rev 2010;74:570-88.

241. Sato YT, Hamada T, Kubo K, Yamada A, Kishida T, Mazda O, et al. Folding transition into a loosely collapsed state in plasmid DNA as revealed by singlemolecule observation. FEBS Lett 2005;579:3095-9.

242. Keatch SA, Su T-J, Dryden DTF. Alleviation of restriction by DNA condensation and non-specific DNA binding ligands. Nucl Acids Res 2004;32:5841-50.

243. Hou Y, Zhang H, Miranda L, Lin S. Serious overestimation in quantitative PCR by circular (supercoiled) plasmid standard: microalgal pcna as the model gene. PLOS ONE 2010;5:e9545.

244. Chen J, Kadlubar FF, Chen JZ. DNA supercoiling suppresses real-time PCR: a new approach to the quantification of mitochondrial DNA damage and repair. Nucl Acids Res 2007;35:1377-88.

245. Ponsaerts P, Van Tendeloo VFI, Berneman ZN. Cancer immunotherapy using RNA-loaded dendritic cells. Clin Exp Immunol 2003;134:378-84.

246. Grünebach F, Müller MR, Brossart P. New developments in dendritic cellbased vaccinations: RNA translated into clinics. Cancer Immunol Immunother 2005;54:517-25. 
247. Zhong L, Granelli-Piperno A, Choi Y, Steinman RM. Recombinant adenovirus is an efficient and non-perturbing genetic vector for human dendritic cells. Eur J Immunol 1999;29:964-72.

248. Metharom P, Ellem KA, Schmidt C, Wei MQ. Lentiviral vector-mediated tyrosinase-related protein 2 gene transfer to dendritic cells for the therapy of melanoma. Hum Gene Ther 2001;12:2203-13.

249. Zarei S, Leuba F, Arrighi J-F, Hauser C, Piguet V. Transduction of dendritic cells by antigen-encoding lentiviral vectors permits antigen processing and MHC class I-dependent presentation. J Allergy Clin Immunol 2002;109:98894.

250. Schroers R, Sinha I, Segall H, Schmidt-Wolf IG, Rooney CM, Brenner MK, et al. Transduction of human PBMC-derived dendritic cells and macrophages by an HIV-1-based lentiviral vector system. Mol Ther 2000;1:171-9.

251. Dyall J, Latouche JB, Schnell S, Sadelain M. Lentivirus-transduced human monocyte-derived dendritic cells efficiently stimulate antigen-specific cytotoxic T lymphocytes. Blood 2001;97:114-21.

252. Tan PH, Beutelspacher SC, Xue S-A, Wang Y-H, Mitchell P, McAlister JC, et al. Modulation of human dendritic-cell function following transduction with viral vectors: implications for gene therapy. Blood 2005;105:3824-32.

253. Tuettenberg A, Jonuleit H, Tüting T, Brück J, Knop J, Enk AH. Priming of T cells with Ad-transduced DC followed by expansion with peptide-pulsed DC significantly enhances the induction of tumor-specific CD8+ $\mathrm{T}$ cells: implications for an efficient vaccination strategy. Gene Ther 2003;10:243-50.

254. Young LS, Mautner V. The promise and potential hazards of adenovirus gene therapy. Gut 2001;48:733-6.

255. Rouas R, Uch R, Cleuter Y, Jordier F, Bagnis C, Mannoni P, et al. Lentiviralmediated gene delivery in human monocyte-derived dendritic cells: optimized design and procedures for highly efficient transduction compatible with clinical constraints. Cancer Gene Ther 2002;9:715-24.

256. Chinnasamy N, Chinnasamy D, Toso JF, Lapointe R, Candotti F, Morgan RA, et al. Efficient gene transfer to human peripheral blood monocyte-derived dendritic cells using human immunodeficiency virus type 1-based lentiviral vectors. Hum Gene Ther 2000;11:1901-9.

257. Gruber A, Kan-Mitchell J, Kuhen KL, Mukai T, Wong-Staal F. Dendritic cells transduced by multiply deleted HIV-1 vectors exhibit normal phenotypes and functions and elicit an HIV-specific cytotoxic T-lymphocyte response in vitro. Blood 2000;96:1327-33.

258. Buechler L. The role of MLL/AF4 in leukaemic cell biology [thesis]. Newcastle upon Tyne: Faculty of Medicine, Newcastle University; 2010. 
259. Dull T, Zufferey R, Kelly M, Mandel RJ, Nguyen M, Trono D, et al. A thirdgeneration lentivirus vector with a conditional packaging system. J Virol 1998;72:8463-71.

260. Pfeifer A. Lentiviral transgenesis. Transgenic Res 2004;13:513-22.

261. Zufferey R, Dull T, Mandel RJ, Bukovsky A, Quiroz D, Naldini L, et al. Selfinactivating lentivirus vector for safe and efficient in vivo gene delivery. $J$ Virol 1998;72:9873-80.

262. Kalejta RF, Brideau AD, Banfield BW, Beavis AJ. An integral membrane green fluorescent protein marker, Us9-GFP, is quantitatively retained in cells during propidium iodide-based cell cycle analysis by flow cytometry. Exp Cell Res 1999;248:322-8.

263. Kalejta RF, Shenk T, Beavis AJ. Use of a membrane-localized green fluorescent protein allows simultaneous identification of transfected cells and cell cycle analysis by flow cytometry. Cytometry 1997;29:286-91.

264. Kusser KL, Randall TD. Simultaneous detection of EGFP and cell surface markers by fluorescence microscopy in lymphoid tissues. $J$ Histochem Cytochem 2003;51:5-14.

265. Marten A, Ziske C, Schottker B, Weineck S, Renoth S, Buttgereit P, et al. Transfection of dendritic cells (DCs) with the CIITA gene: increase in immunostimulatory activity of DCs. Cancer Gene Ther 2001;8:211-9.

266. Andreakos E, Smith C, Monaco C, Brennan FM, Foxwell BM, Feldmann M. Ikappa B kinase 2 but not NF-kappa B-inducing kinase is essential for effective DC antigen presentation in the allogeneic mixed lymphocyte reaction. Blood 2003;101:983-91.

267. Taylor SS, Haste NM, Ghosh G. PKR and eIF2alpha: integration of kinase dimerization, activation, and substrate docking. Cell 2005;122:823-5.

268. Aste-Amezaga M, Ma X, Sartori A, Trinchieri G. Molecular mechanisms of the induction of IL-12 and its inhibition by IL-10. J Immunol 1998;160:5936-44. 